Andrews University

Digital Commons @ Andrews University

\title{
A Model Of Distress Tolerance In Self-Damaging Behaviors: Examining The Role Of Emotional Reactivity And Learned Helplessness
}

Brittany Kay Sommers

Andrews University, sommersb@andrews.edu

Follow this and additional works at: https://digitalcommons.andrews.edu/dissertations

Part of the Social and Behavioral Sciences Commons

\section{Recommended Citation}

Sommers, Brittany Kay, "A Model Of Distress Tolerance In Self-Damaging Behaviors: Examining The Role Of Emotional Reactivity And Learned Helplessness" (2017). Dissertations. 1633.

https://digitalcommons.andrews.edu/dissertations/1633

https://dx.doi.org/10.32597/dissertations/1633

This Dissertation is brought to you for free and open access by the Graduate Research at Digital Commons @ Andrews University. It has been accepted for inclusion in Dissertations by an authorized administrator of Digital Commons@ Andrews University. For more information, please contact repository@andrews.edu. 


\begin{abstract}
A MODEL OF DISTRESS TOLERANCE IN SELF-DAMAGING BEHAVIORS: EXAMINING THE ROLE OF EMOTIONAL REACTIVITY AND LEARNED HELPLESSNESS
\end{abstract}

\author{
by \\ Brittany Kay Sommers
}

Chair: Ronald D. Coffen 


\section{ABSTRACT OF GRADUATE STUDENT RESEARCH}

Dissertation

Andrews University

School of Education

\section{Title: A MODEL OF DISTRESS TOLERANCE IN SELF-DAMAGING BEHAVIORS: EXAMINING THE ROLE OF EMOTIONAL REACTIVITY AND LEARNED HELPLESSNESS}

Name of researcher: Brittany Kay Sommers

Name and degree of faculty chair: Ronald D. Coffen, Ph.D.

Date completed: June 2017

\section{Problem}

Although the literature is clear that low emotional distress tolerance is associated with a myriad of self-damaging behaviors, very little is known about individual difference factors in distress tolerance. Both theoretical and empirical support suggest that emotional reactivity and learned helplessness may be individual difference factors in distress tolerance. Specifically, individuals with high emotional reactivity and high learned helplessness may be at risk for low distress tolerance. Further research was needed to clarify the role of emotional reactivity and learned helplessness in distress tolerance in the context of self-damaging behaviors. 


\section{Method}

Participants completed surveys which measured their (a) emotional reactivity, (b) learned helplessness, (c) distress tolerance, (d) two-week frequency of self-damaging behaviors, and (e) lifetime frequency of self-damaging behaviors. Structural equation modeling was used to test two models for the role of emotional reactivity and learned helplessness in distress tolerance. The first model was in the context of two-week frequency of self-damaging behaviors and the second model was in the context of lifetime frequency of self-damaging behaviors.

Results

Structural equation modeling indicated that the original models were a poor fit for the data. So, both models were revised on the basis of theory and modification indices. The revised models revealed that emotional reactivity and learned helplessness had negative direct effects on distress tolerance. Together, emotional reactivity and learned helplessness explained $70 \%$ of the observed variance in distress tolerance. Distress tolerance had a negative direct effect on two-week frequency of self-damaging behaviors, explaining $7 \%$ of the observed variance. Distress tolerance had a negative direct effect and depression had a positive direct effect on lifetime frequency of self-damaging behaviors, together explaining $36 \%$ of the observed variance.

\section{Conclusions}

This study confirmed emotional reactivity and learned helplessness as important individual difference factors in emotional distress tolerance. It suggests that high emotional reactivity and high learned helplessness contribute to low distress tolerance. 
This study also demonstrated that distress tolerance explains a small amount of variance in two-week frequency of self-damaging behaviors. Whereas, distress tolerance together with depression explains a larger amount of variance in lifetime frequency of selfdamaging behaviors. These results have implications for researchers studying distress tolerance and self-damaging behaviors, clinicians treating clients with difficulty managing distress or with self-damaging behaviors, and individuals developing preventative initiatives to reduce the development of self-damaging behaviors. In particular, this study suggests that emotional reactivity may be an important target of clinical intervention and preventative education. 
Andrews University

School of Education

\title{
A MODEL OF DISTRESS TOLERANCE IN SELF-DAMAGING BEHAVIORS: EXAMINING THE ROLE OF EMOTIONAL REACTIVITY AND LEARNED HELPLESSNESS
}

\author{
A Dissertation \\ Presented in Partial Fulfillment \\ of the Requirements for the Degree \\ Doctor of Philosophy
}

by

Brittany Kay Sommers

June 2017 
@ C Copyright by Brittany Kay Sommers 2017 All Rights Reserved 


\title{
A MODEL OF DISTRESS TOLERANCE IN SELF-DAMAGING BEHAVIORS: EXAMINING THE ROLE OF EMOTIONAL REACTIVITY AND LEARNED HELPLESSNESS
}

\author{
A dissertation \\ presented in partial fulfillment \\ of the requirements for the degree \\ Doctor of Philosophy
}

by

Brittany Kay Sommers

APPROVAL BY THE COMMITTEE:

Chair: Ronald D. Coffen

Member: Bradly K. Hinman

Member: Tevni E. Guerra Grajales
Dean, School of Education

Robson M. Marinho

External: Anne Carpenter

Date approved 
To God be the glory, great things He has done! 


\section{TABLE OF CONTENTS}

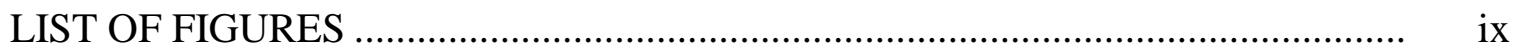

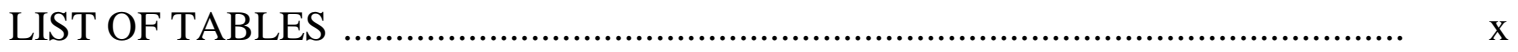

LIST OF ABBREVIATIONS ............................................................................ xi

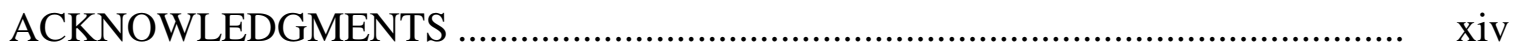

\section{Chapter}

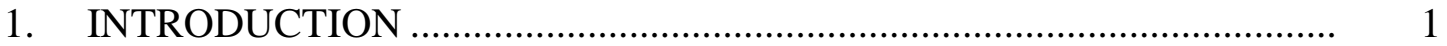

Background of the Problem................................................................. 1

Statement of the Problem ...................................................................... 5

Purpose of the Study .......................................................................... 6

Hypotheses and Research Questions ..................................................... 6

Rationale.............................................................................. 8

Conceptual Framework ................................................................... 10

Importance of the Study ……………............................................... 12

Definition of Terms .......................................................................... 13

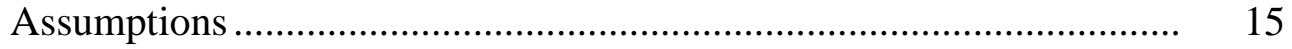

General Methodology .................................................................... 16

Limitations ................................................................................. 17

Delimitations ............................................................................. 20

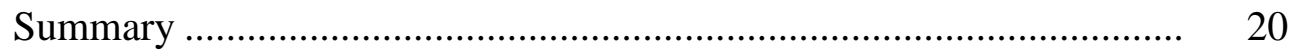

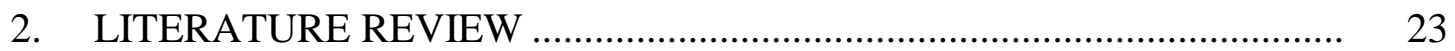

Background to the Problem..................................................................... 23

Distress Tolerance Introduction ......................................................... 24

Definition and Terminology of DT ………………………........ 25

Inclusion in Literature Review..................................................... 26

Measures of DT Overview ............................................................ 27

Factor Structure of Broad Distress Tolerance ……………............. 28

Theoretical Framework .................................................................... 31

Linehan's BioSocial Theory of Emotion Dysregulation.................. 31

Linehan's Model of DT Skills Training.......................................... 33

Lynch and Mizon's Three-Factor Model of DT ............................ 34

Lynch and Mizon's Proposed Individual Difference Factors

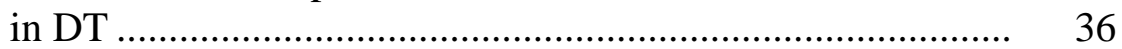

My Theoretical Model of Individual Difference Factors in DT .... 37 
Emotional DT Overview ................................................................ 38

Factor Structure of Emotional DT............................................... 38

General Research on Emotional DT ............................................ $\quad 40$

Stability of DT over Time................................................... 40

Longitudinal Study in Early Adolescents ......................... 40

Longitudinal Studies in Adults ....................................... 41

Evidence Supporting Modification Through Treatment... 43

Evidence Opposing Modification Through Treatment ...... 46

Relationship Between DT and Related Concepts ................... 47

Relationship Between DT and Avoidance........................ 47

Relationship Between DT and Urgency .......................... 49

Factors Known to Influence DT …………………............... 51

The Influence of Emotion Regulation and Attentional

Control

51

The Influence of Mindfulness and Rumination ................ 53

The Influence of Personality Traits................................... 54

The Influence of Parenting............................................. 58

The Influence of Biological Factors ................................ 59

Emotional DT Summary ............................................................ 61

Emotional Reactivity Overview ........................................................... $\quad 62$

History and Definition of Emotional Reactivity .......................... 62

Emotional Reactivity Factor Structure..................................... 63

Relationship Between Emotional Reactivity and

Psychological Disorders................................................... 64

Evidence Supporting Emotional Reactivity as an Individual

Difference Factor in DT ...................................................... 66

DT and Emotional Reactivity in Borderline Symptoms ......... 67

DT and Emotional Reactivity in Depression Symptoms ......... 68

DT and Emotional Reactivity in Heavy Drinking .................. $\quad 70$

DT and Emotional Reactivity in NSSI................................... 71

DT and Emotional Reactivity in Obsessive-Compulsive Symptoms ……........................................................... 72

DT and Emotional Reactivity in Panic Symptoms ................. 74

DT and Emotional Reactivity in Research Response .............. $\quad 75$

DT and Emotional Reactivity in Worry ................................... 76

Evidence Opposing Emotional Reactivity as an Individual

Difference Factor in DT ....................................................... 77

DT and Emotional Reactivity in Anxiety ............................... 77

DT and Emotional Reactivity in Smoking .............................. 79

Emotional Reactivity Summary .................................................. 80

Learned Helplessness Overview ....................................................... $\quad 80$

History and Definition of Learned Helplessness .......................... $\quad 80$

Learned Industriousness-Helplessness Continuum.......................... 83

Evidence Supporting Learned Helplessness ........................... 85

Evidence Supporting Learned Industriousness ........................ 86

Learned Industriousness-Helplessness Continuum Summary ........ 88 
Evidence Supporting Learned Helplessness as an Individual Difference Factor in DT .................................................... 90

Learned Helplessness and Physical Pain Tolerance ............... 90

Emotion Regulation and Helplessness in NSSI ..................... 91

Evidence Opposing Learned Helplessness as an Individual

Difference Factor in DT ................................................... 92

Learned Helplessness and Physical Pain Tolerance ............... 92

DT and Negative Reinforcement ........................................ 93

Learned Helplessness Summary ............................................... 95

Relationship Between DT and Covariates ....................................... 95

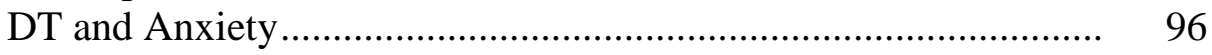

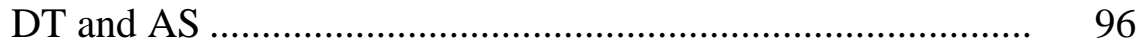

DT and Health Anxiety .................................................... 97

DT and Obsessive Compulsive Anxiety ............................. 97

DT and Panic................................................................ 97

DT and Social Anxiety................................................... 98

DT and Trait Anxiety..................................................... 98

DT and Worry ............................................................. 99

DT and Depression .......................................................... 100

DT, AS, and Depression ............................................... 100

DT Subscales and Depression............................................ 102

DT and Covariates Summary .................................................... 104

Relationship Between Distress Tolerance and Self-Damaging

Behaviors ............................................................................ 104

DT and Eating Disorders....................................................... 105

The Role of cDTS: Avoidance of Affect .............................. 105

The Role of cDTS: Behavioral Avoidance of Positive Affect ...................................................................... 107

The Role of DT in Anorexia Nervosa................................... 111

The Role of DT in Binge Eating and Overeating ................... 112

The Role of DT in Bulimia Nervosa.................................... 114

DT and NSSI ................................................................... 116

Role of DT in NSSI Lifetime Frequency ............................ 116

Role of DT in Number of NSSI Methods ............................. 117

Evidence from Treatment Outcomes ..................................... 118

DT and Suicide.................................................................... 119

Role of DT in Suicidal Desire.............................................. 119

Role of DT in Pain Tolerance ............................................. 120

Role of DT in Suicidal Ideation .......................................... 121

Role of DT in Past Suicide Attempts .................................. 122

Role of DT in Suicide Potential ........................................... 122

Role of DT in Acquired Capability for Suicide ..................... 122

DT, NSSI, and Suicide Considered Together ............................ 124

DT and Self-Damaging Behaviors Summary ............................. 126

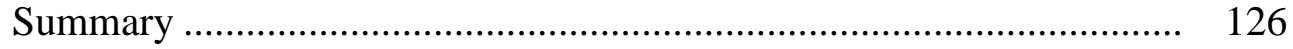




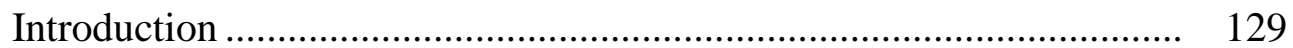

Research Design .............................................................................. 129

Population and Sample.................................................................... 131

Hypotheses and Research Questions ................................................. 134

Variables Definition ....................................................................... 136

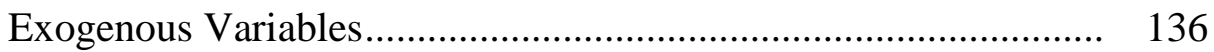

Endogenous Variables................................................................... 139

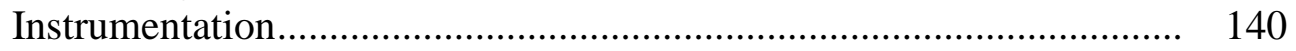

Depression Anxiety Stress Scales ............................................. 140

Distress Tolerance Scale .............................................................. 143

Emotion Reactivity Scale ............................................................. 145

Learned Helplessness Scale ....................................................... 146

Self-Damaging Behaviors Questionnaire....................................... 147

SITBI............................................................... 148

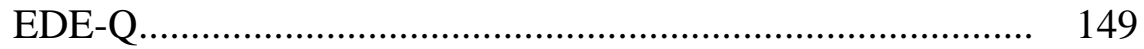

Self-Damaging Behaviors Questionnaire …………….......... 150

Data Collection ........................................................................... 152

Treatment of Data.............................................................................. 157

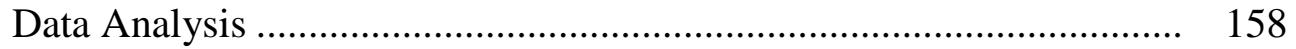

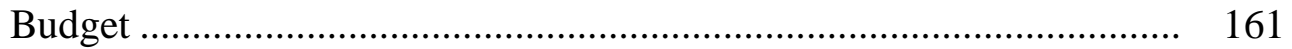

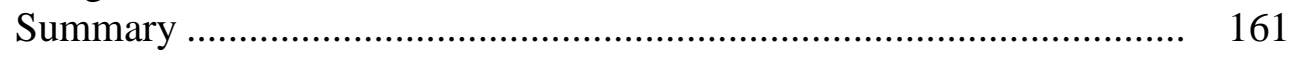

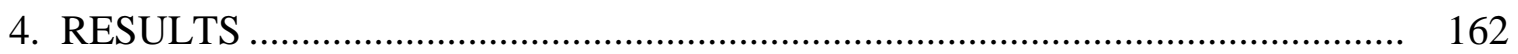

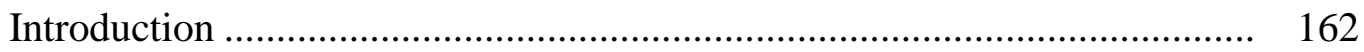

Description of the Sample ..................................................................... 162

Discontinued Responses................................................................... 163

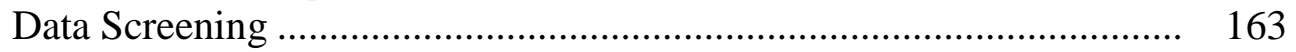

Participants Description .................................................................. 164

Instrument Reliability .......................................................................... 166

Emotion Reactivity Scale Item Reduction .............................................. 167

Variables Description ............................................................................ 167

Normality ............................................................................. 167

Variable Means and Standard Deviations .......................................... 168

Endorsement of Self-Damaging Behaviors .......................................... 170

Variable Description by Demographic Characteristics ......................... 171

Zero-Order Correlations .................................................................... 173

Hypotheses Testing ............................................................................. 181

Two-Week Frequency Model................................................................. 182

Original Two-Week Model ....................................................... 182

Fitted Two-Week Model ............................................................... 184

Intercorrelations Among Variables ................................................ 185

Lifetime Frequency Model................................................................. 187

Original Lifetime Model ......................................................... 187 
Fitted Lifetime Model ............................................................. 187

Summary ................................................................................ 190

5. SUMMARY, DISCUSSION, AND IMPLICATIONS ...................................... 192

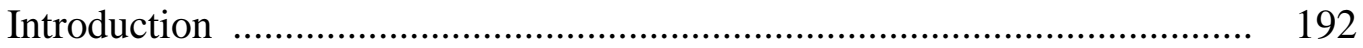

Purpose of the Study ........................................................................... 192

Summary of the Literature Review ............................................................. 193

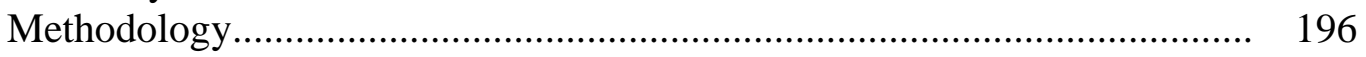

Findings and Discussion......................................................................... 197

Respondent's Demographic Characteristics........................................ 197

Incidence of Self-Damaging Behaviors ................................................ 198

Variable Description by Demographic Characteristics ......................... 199

Zero-Order Correlations .................................................................. 200

Two-Week Model Fit ........................................................................... 201

Fitted Two-Week Model ................................................................ 203

Lifetime Model Fit ....................................................................... 205

Lifetime Fitted Model ........................................................................ 207

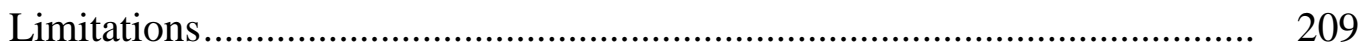

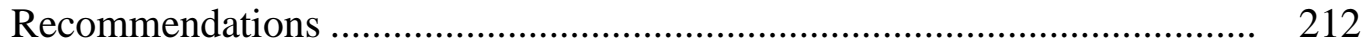

Recommendations for Practice......................................................... $\quad 212$

Recommendations for Future Research .............................................. 215

Summary ................................................................................ 218

Appendix

A. STUDIES OF THE RELATIONSHIP BETWEEN DT AND

SELF-DAMAGING BEHAVIORS ................................................. 219

B. APPROVAL LETTERS ...................................................................... 237

C. INFORMED CONSENT ................................................................ 240

D. DEMOGRAPHIC QUESTIONNAIRE ...................................................... 243

E. SURVEYS ................................................................................. 245

F. OUTPUT FOR FITTED TWO-WEEK MODEL_........................................ 255

G. OUTPUT FOR FITTED LIFETIME MODEL …….................................... 261

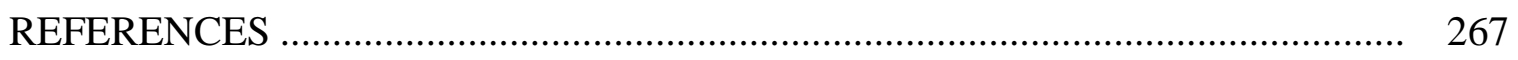

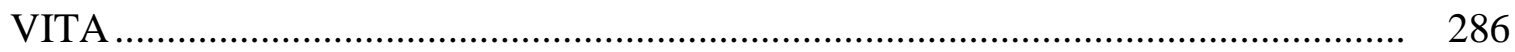




\section{LIST OF FIGURES}

1. Hypothesized Two-Week Frequency Model .......................................... 7, 135

2. Hypothesized Lifetime Frequency Model ............................................ 9, 137

3. Fitted Two-Week Model..................................................................... 184

4. Fitted Lifetime Model ............................................................................ 188 


\section{LIST OF TABLES}

1. Conceptual Definitions of Variables and Source of Associated Survey Items .... 141

2. Adaptations and Combinations of Self-Damaging Behaviors Questionnaire Items

3. Respondents' Demographic Characteristics

4. Internal Reliability

5. Variable Means and Standard Deviations

6. Respondents Endorsing Each Self-Damaging Behavior

7. Emotional Reactivity, Learned Helplessness, and DT by Demographic Characteristics

8. Two-Week Frequency of Self-Damaging Behaviors by Demographic Characteristics

9. Lifetime Frequency of Self-Damaging Behaviors by Demographic Characteristics

10. Zero-Order Correlations

11. Chi-Square and Goodness of Fit Indices for Hypothesized and Respecified Models

12. Summary of Causal Effects of Fitted Two-Week Model

13. Raw and Standardized Coefficients for the Revised Two-Week Model

14. Summary of Causal Effects of Fitted Lifetime Model

15. Raw and Standardized Coefficients for the Revised Lifetime Model 


\section{LIST OF ABBREVIATIONS}

\begin{tabular}{|c|c|}
\hline $\mathrm{ACT}$ & Acceptance and commitment therapy \\
\hline AN & Anorexia nervosa \\
\hline AS & Anxiety sensitivity \\
\hline ASPD & Antisocial personality disorder \\
\hline $\mathrm{BHD}$ & Breath Holding Duration Task \\
\hline $\mathrm{BPD}$ & Borderline personality disorder \\
\hline CBT & Cognitive behavioral therapy \\
\hline cDTS & Distress Tolerance Scale, 2007 \\
\hline CFA & Confirmatory factor analysis \\
\hline CFI & Comparative fit index \\
\hline DASS-21 & Depression Anxiety Stress Scales 21 \\
\hline DI & Discomfort intolerance \\
\hline DII & Distress Intolerance Index \\
\hline DIS & Discomfort Intolerance Scale \\
\hline DBT & Dialectical behavior therapy \\
\hline$D S M-5$ & The Diagnostic and Statistical Manual of Mental Disorders, $5^{\text {th }}$ edition \\
\hline DT & Emotional distress tolerance \\
\hline DTT & Distress Tolerance Test \\
\hline ED & Eating disorder \\
\hline EDE-Q & Eating Disorder Exam Questionnaire 6.0 \\
\hline
\end{tabular}




\begin{tabular}{|c|c|}
\hline EFA & Exploratory factor analysis \\
\hline ERS & Emotion Reactivity Scale \\
\hline ETS & Emotional Tolerance Scale \\
\hline FDS & Frustration Discomfort Scale \\
\hline FT & Frustration tolerance \\
\hline GFI & Goodness-of-fit index \\
\hline HEDs & Heavy episodic drinking youth \\
\hline IU & Intolerance of uncertainty \\
\hline $\mathrm{K}-\mathrm{SADS}-\mathrm{PL}$ & Schedule for Affective Disorders and Schizophrenia for School-Age \\
\hline & Children-Present and Lifetime Version \\
\hline LHS & Learned Helplessness Scale \\
\hline MDD & Major depressive disorder \\
\hline МTPT & Mirror-tracing Persistence Task \\
\hline MTPT-C & Computerized Mirror-tracing Persistence Task \\
\hline NFI & Normed-fit index \\
\hline NSSI & Nonsuicidal self-injury \\
\hline PANAS & Positive and Negative Affect Schedule \\
\hline PASAT-C & Computerized Paced Auditory Serial Addition Test \\
\hline $\mathrm{RCI}$ & Reliable change index \\
\hline RMSEA & Root mean square error of approximation \\
\hline sDTS & Distress Tolerance Scale, 2005 \\
\hline SEM & Structural equation modeling \\
\hline SIDI & Skill for Improving Distress Intolerance \\
\hline
\end{tabular}


SITBI Self-Injurious Thoughts and Behaviors Interview - Long Form

SUD Substance use disorder

TA Tolerance of ambiguity

TNASS Tolerance of Negative Affective States Scale 


\section{ACKNOWLEDGMENTS}

This project was inspired by friends and clients who have shared their experiences of self-damaging behaviors. Thank you for bravely living and telling your stories!

This project was possible only with the support of many others. Firstly, thanks to my nuclear and extended family. I treasured your encouragement, humor, insight, patience, prayers, and unwavering belief in me throughout this process. I am specifically thankful to mom and dad for your financial support of this research and to Bud and Kate for serving as my editors.

Secondly, I am thankful to my colleagues and friends. Thanks to those in the program for providing both academic and emotional support. A few in particular: Salenna, I am tremendously grateful to have had a sister for this journey, and I cannot imagine the countless hours without you by my side. Kristina, I am thankful for your guidance and friendship, having you always a step ahead has provide much hope and practical help. Thanks also to my colleagues and supervisors at Southwestern Counseling and Psychological Services and Cleveland State University's Counseling Center for supporting my progress throughout.

Thirdly, thanks to the authors of the instruments used in this project for your clarifying correspondence and gracious permission to use your instruments. Thanks also to Dr. James Gaskin for posting your helpful structural equation modeling courses online. I am grateful to Dr. Carole Woolford-Hunt for your help in the initial brainstorming and framing of my dissertation. 
Lastly, but importantly, my deepest thanks to my committee: Dr. Ronald Coffen, I so appreciate the consistency, thoroughness, and enthusiasm of your involvement. I know you have nearly as many hours invested in this project as I do, and I am very grateful. I am honored to have learned from your feedback and mentorship throughout. Dr. Tevni Grajales Guerra and Dr. Bradly Hinman, I am grateful for your guidance in building and executing this research. Thank you! 


\section{CHAPTER 1}

\section{INTRODUCTION}

\section{Background of the Problem}

Emotional distress tolerance (DT) is defined as one's perceived ability to withstand negative emotional states (Simons \& Gaher, 2005; Zvolensky, Leyro, Bernstein, \& Vujanovic, 2011). Distress tolerance is a concept with importance across multiple diagnostic categories (Bardeen, Fergus, \& Orcutt, 2013; Kiselica, Webber, \& Bornovalova, 2014). In particular, low DT has been linked to behaviors that often immediately reduce the individual's distress, but which may cause physical and psychological harm over the long run by avoiding real-life issues or problems (McHugh et al., 2014). Behaviors which have been linked with low DT include eating disorders (EDs), nonsuicidal self-injury (NSSI), and suicidality (Anestis, Pennings, Lavender, Tull, \& Gratz, 2013; Anestis, Selby, Fink, \& Joiner, 2007). These symptoms are important for study since they cause physical harm to individuals engaging in them and also since individuals with these self-damaging behaviors are frequent consumers of clinical services. While this study examined only a subset of self-damaging behaviors, individuals who engage in these self-damaging behaviors may be included in broader diagnostic categories.

For instance, individuals engaging in restricting behaviors may meet criteria for anorexia nervosa (AN). According to the American Psychiatric Association (2013), the 
12-month prevalence rate for $\mathrm{AN}$ in females is $0.4 \%$. The prevalence rate is unknown in males, but the disorder is known to be less prevalent in males. Anorexia Nervosa is associated with a host of serious consequences including social problems, academic problems, career problems, health problems, and death. Anorexia Nervosa is associated with a $5 \%$ crude mortality rate per decade. These deaths result both from medical complications of the disorder (e.g., from an unsustainable body weight, from heart complications) and from elevated suicide risk.

Individuals engaging in purging behaviors may meet criteria for bulimia nervosa. According to the American Psychiatric Association (2013), the 12-month prevalence rate for bulimia nervosa in females is $1 \%$ to $1.5 \%$. The prevalence rate is unknown for males, but the disorder is known to be less prevalent in males. The crude mortatlity rate for individuals with bulimia nervosa is also elevated with a $2 \%$ rate per decade. While the severity of functional impairment is often less in bulimia nervosa than in AN, individuals with bulimia nervosa still experience social problems, health problems, and risk death from health complications (e.g., from choking during a purging event, from throat cancer) or elevated suicide risk.

Individuals engaging in binge-eating behaviors may meet criteria for binge eating disorder. According to the American Psychiatric Association (2013), the 12-month prevalence rate for binge-eating disorder in females is $1.6 \%$ and the prevalence rate in males is $0.8 \%$. Binge-eating disorder is associated with social problems, health problems, and increased utilization of health care services. In some cases, binge-eating disorder is associated with weight gain and obesity which carry their own funcitonal consequences and health risks. 
Individuals who engage in NSSI may meet criteria for nonsuicidal self-injury disorder. This disorder is listed as a condition for further study in The Diagnostic and Statistical Manual of Mental Disorders (5th ed.; DSM-5; American Psychiatric Association, 2013). Due to variance in operational definitions of NSSI, reported prevalence rates vary greatly (see Shaffer \& Jacobson, 2010 for a review). As summarized by Washburn et al. (2012), lifetime prevalence of NSSI in nonclinical samples is estimated to be between $7.5 \%$ and $23 \%$. In clinical samples, the reported prevalence rates vary between $12 \%$ and $82 \%$. Additionally, the prevalence rates of NSSI are thought to be increasing (Ferrara, Terrinoni, \& Williams, 2012; Purington \& Whitlock, 2010). In clinical populations, females have higher rates of NSSI than males. However, in nonclinical settings there are similar rates between males and females (Andover, Primack, Gibb, \& Pepper, 2010). Functional consequences associated with NSSI include social problems, health problems due to the behaviors (e.g., blood loss, infected wounds), and elevated suicide risk. Although NSSI is a phenomenon distinct from suicide (Muehlenkamp \& Kerr, 2010), it is a strong predictor of a suicide attempt (Ferrara et al., 2012). For example, among inpatient adults, Andover and Gibb (2010) found that both NSSI history and NSSI frequency predicted suicide attempts as well as current suicidal ideation predicted suicide attempts. Nonsuicidal self-injury history and NSSI frequency also predicted suicide attempts better than depression, hopelessness, and Borderline Personality Disorder (BPD) characteristics predicted suicide attempts.

Individuals with suicidal ideation or history of a suicide attempt may meet criteria for a depressive disorder. According to the American Psychiatric Association (2013), the 12-month prevelance of major depressive disorder (MDD) is 7\%. In 2015, 3.9\% of the 
USA population reported suicidal thoughts and $1.1 \%$ reported created a suicide plan (Center for Disease Control, 2015). MDD is more prevalent among females than males (American Psychiatric Association, 2013), and suicidal ideation is also more prevalent among females than males (Center for Disease Control, 2015). Functional consequences of depressive disorders range in severity but can include difficulties with self-care, social problems, academic problems, career problems, health problems, increased utilization of health care services, and elevated suicide risk.

Individuals with a history of a suicide attempt may also meet criteria for suicidal behavior disorder (listed as a condition for further study in the DSM-5). In 2015, suicide was the $10^{\text {th }}$ leading cause of death in the USA (Center for Disease Control, 2015). Also in 2015 , suicide was attempted by $0.6 \%$ of individuals over the age of 18 . In addition to the loss of life, suicide results in medical and work-loss costs totaling approximately $\$ 51$ billion annually. The risk of a suicide attempt is higher among women, but the risk of suicide completion is higher among men (American Psychiatric Association, 2013). Of individuals who make an unsuccessful suicide attempt, 25-30\% will eventually make another attempt (American Psychiatric Association, 2013). While death is the most severe consequence of suicide attempts, uncompleted suicide attempts can result in lifelong health problems and disabilities.

Clearly, self-damaging behaviors respresent a significant and costly public health concern and also pose severe functional consequences for the individuals engaging in the behaviors. Since DT is associated with these self-damaging behaviors, DT is a crucial construct for research and clinical focus. Distress tolerance is thought to be malleable in response to clinical intervention (Marshall et al., 2008). As such, DT skills training is 
incorporated in a variety of therapeutic approaches including Cognitive Behavioral Therapy (CBT), Dialectical Behavior Therapy (DBT), Acceptance and Commitment Therapy (ACT), and DT-specific approaches. However, factors accounting for individual differences in level of DT remain mostly unidentified (G. Feldman, Dunn, Stemke, Bell, \& Greeson, 2014). Individual difference factors refer to "ways in which people differ from one another" (Greenberg, 2011, p. 134), such as differences between people in personality traits, self-concept, physiological responses, sociability, risk taking, personal interests, values, attitudes, etc. This "absence of knowledge about factors that account for individual differences in DT hinders efforts to target and increase DT" (Marshall et al., 2008, p. 2).

\section{Statement of the Problem}

Although the literature is clear that low DT is associated with a myriad of selfdamaging behaviors, very little is known about individual difference factors in DT. Both theoretical and empirical support (e.g., Ellis, Fischer, \& Beevers, 2010; Nock \& Mendes, 2008; Winward, Bekman, Hanson, Lejuez, \& Brown, 2014) suggest that emotional reactivity may be an individual difference factor in DT. Theoretical support and empirical evidence from related constructs (e.g., Slee, Garnefski, Spinhoven, \& Arensman, 2008; Yamamoto et al., 2010) suggest that learned helplessness may also be an individual difference factor in DT. Specifically, individuals with high emotional reactivity and high learned helplessness may be at risk for low DT. Further research is needed to clarify the role of emotional reactivity and learned helplessness in DT in the context of selfdamaging behaviors. 


\section{Purpose of the Study}

The first purpose of this study was to review current literature about DT, individual difference factors in DT, and the relationship between DT and self-damaging behaviors. The second purpose of this study was to test two models of individual difference factors in DT in the context of self-damaging behaviors (fasting, restricting, binging, purging, NSSI, suicidal ideation, and suicide attempts). In particular, I sought to investigate the role of two potential individual difference factors in DT - emotional reactivity and learned helplessness. The findings from this study contribute to the literature on DT and to the knowledge of the relationship between DT and self-damaging behaviors.

\section{Hypotheses and Research Questions}

The first hypothesis of this study was that the reproduced covariance matrices $\Sigma(\gamma)$ implied in the two-week frequency theoretical model (Figure 1) and the observed sample covariance matrices $S$ were equal. This hypothesis addressed the following research question: is the hypothesized two-week frequency model a good fit to the sample? As depicted, the two-week frequency model proposed a direct effect between emotional reactivity and DT, between learned helplessness and DT, and between DT and two-week frequency of self-damaging behaviors. It also proposed an indirect effect between emotional reactivity and two-week frequency of self-damaging behaviors and between learned helplessness and two-week frequency of self-damaging behaviors. 


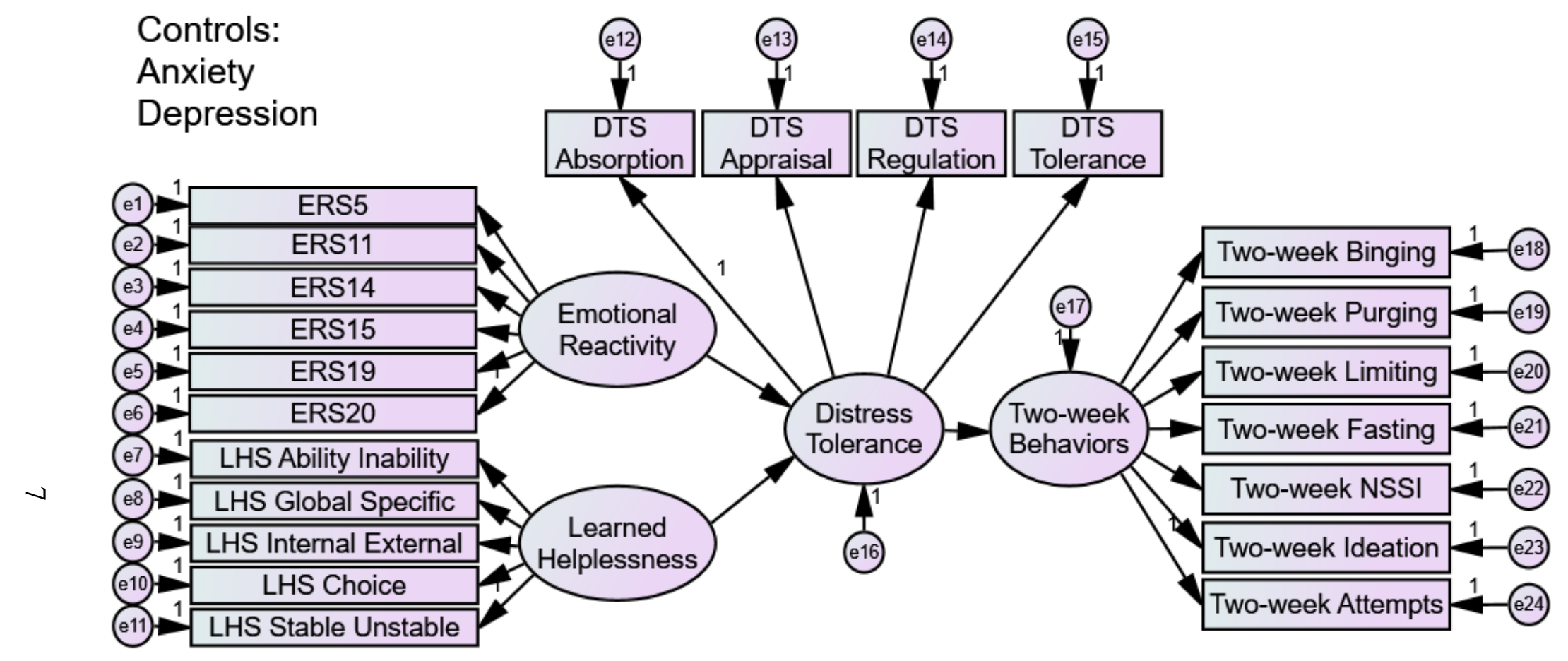

Figure 1: Hypothesized Two-Week Frequency Model 
The second hypothesis of this study was that the reproduced covariance matrices $\Sigma(\gamma)$ implied in the lifetime frequency theoretical model (Figure 2) and the observed sample covariance matrices $S$ were equal. This hypothesis addressed the following research question: is the hypothesized lifetime frequency model a good fit to the sample? As depicted, the lifetime frequency model proposed a direct effect between emotional reactivity and DT, between learned helplessness and DT, and between DT and lifetime frequency of self-damaging behaviors. It also proposed an indirect effect between emotional reactivity and lifetime frequency of self-damaging behaviors and between learned helplessness and lifetime frequency of self-damaging behaviors.

\section{Rationale}

Increased knowledge about factors influencing DT may better inform and refine DT intervention efforts which will in turn enhance clients' quality of life. For instance, knowing which factors influence DT may help to highlight which aspects are responsible for the effectiveness of existing interventions that have been found to increase DT. Or, knowing which factors influence DT may serve as a platform for adding elements to existing interventions to enhance the effectiveness of the interventions to increase DT.

Learning about contributing factors may also inform the development of prevention programs aimed at building DT before clinical symptoms develop. Prevention programs which teach DT skills may reduce the burden of low DT for individuals (e.g., greater ability to cope with negative emotions, greater ability to maintain independent living, greater ability to maintain a job). Prevention programs may also reduce the burden of low DT for society (e.g., increased citizen productivity, decreased money spent on clinical treatments). 
Controls:

Age

Anxiety

Depression

6

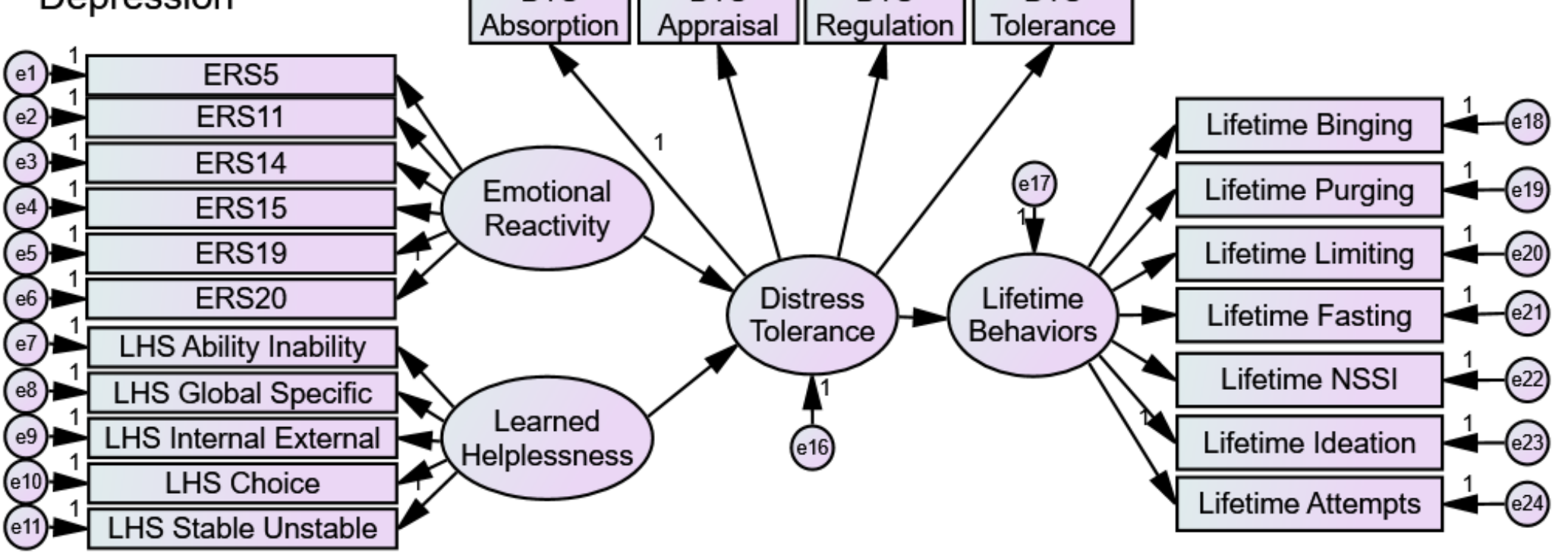

Figure 2. Hypothesized Lifetime Frequency Model 
Further knowledge of DT's development and the relationship between DT and self-damaging behaviors may deepen our understanding of the etiology of self-damaging behaviors as well as our understanding of, and thus the effectiveness of, interventions for these behaviors (Cummings et al., 2013, p. 735). Greater knowledge about which selfdamaging behaviors are related to DT may inform clinicians' selection of DT interventions for a client. Also, if DT is associated with several of the self-damaging behaviors, this knowledge may provide a clearer intervention path for treating selfdamaging behaviors when there is co-morbidity between the symptoms (e.g., between NSSI and EDs).

\section{Conceptual Framework}

Linehan (2015) developed a biosocial theory and a corresponding therapy (DBT) aimed at understanding and increasing an individual's emotional and behavioral regulation. She held that poor emotional regulation results from a combination of biological vulnerabilities (nature) and invalidating childhood environments (nurture). She proposed that one of the biological vulnerabilities at play is emotional vulnerability which involves an individual experiencing emotions more frequently, more intensely, and for longer periods of time than individuals who are not emotionally vulnerable. Lynch and Mizon (2011) similarly proposed that DT level results from the interaction between the influences of nature (biological vulnerabilities) and nurture (sociobiographic feedback and situational factors). One of the biological vulnerabilities they proposed is that high emotional reactivity influences the amount of distress an individual will experience and it can thus "accentuate any tendencies toward maladaptive coping" (Lynch \& Mizon, 2011, p. 60). As both Linehan (2015) and Lynch and Mizon (2011) proposed, I hypothesized 
that emotional reactivity is an individual difference factor in DT. That is, I hypothesized that a high level of emotional reactivity would be associated with low DT.

Lynch and Mizon (2011) further proposed that high emotional reactivity combines with a low level of learned industriousness to produce distress intolerant behavior. If an individual has a learning history in which he/she was rewarded for low effort, avoidance, or escape responses (low learned industriousness), he/she will be unlikely to persist in tolerating distress, which requires high effort. I similarly hypothesized that learned industriousness is also an individual difference factor in DT. That is, low learned industriousness (measured in this study as high learned helplessness; see discussion in chapter 2) would be associated with low DT. However, whereas Lynch and Mizon spoke of learned helplessness as a nature factor, I conceptualized learned helplessness as an individual agency or effort factor. That is, an individual with high learned helplessness will expend low or no effort toward emotion regulation or problemsolving, while an individual with low learned helplessness will expend high effort toward emotion regulation or problem-solving.

Therefore, I hypothesized that both high emotional reactivity and high learned helplessness contribute to low DT. I examined this hypothesized relationship specifically in the context of self-damaging behaviors. I conceptualize each of the self-damaging behaviors as related, at least in part, to low DT. I view the self-damaging behaviors as harmful means of achieving a worthy goal of reducing negative emotional states. While I understand that the self-damaging behaviors may temporarily reduce distress, I hold that the long-term risks of the behaviors outweigh the benefits of the immediate negative reinforcement. I conceptualize intervention approaches which teach acceptance and 
tolerance of negative emotional states as means of reframing and reducing emotional distress as helpful alternatives to self-damaging behaviors.

\section{Importance of the Study}

To date, no existing study could be found that has examined emotional reactivity and learned helplessness together with DT. The broader literature exploring potential individual difference factors in DT is also limited, with results explaining little of the variance in DT. As such, a theoretical model for predictors of DT is lacking. This hinders further research, the development of prevention educational programs, and the refinement of clinical interventions. This study was needed as a building block for understanding individual difference factors in DT and how they are associated with self-damaging behaviors. Strong theoretical models may serve as the foundation for greater knowledge of the development and modulation of DT. This increased understanding is necessary in order to assist individuals with low DT who are engaging in self-harming behaviors to increase their level of DT and learn alternative behaviors which are not harmful. In particular, if a relationship exists between low DT and increased suicidal ideation, a greater understanding of DT could serve to strengthen life-saving interventions among individuals with suicidal ideation and a history of suicide attempts.

Though more literature has examined the relationship between DT and selfdamaging behaviors, the vast majority of the studies have been conducted with either undergraduate or clinical populations. Studying these behaviors in a nonclinical community sample will help to identify if the patterns found among students and in clinical populations extend to a community sample. This is particularly important since 
individuals engaging in self-damaging behaviors may not seek clinical care (e.g., NSSI, American Psychiatric Association, 2013; Evans, Hawton, \& Rodham, 2005).

Of the existing studies, very few have utilized structural equation modeling (SEM) to analyze results. Structural equation modeling is a superior statistical method for testing theoretical models because it allows for the evaluation of multiple independent and dependent variables. This is important in behavioral research since we study multivariate phenomena which are not represented well by univariate or bivariate statistical techniques (Buhi, Goodson, \& Neilands, 2007). Structural equation modeling also allows for simultaneous evaluation of all the variables in the model, which protects against inflation of experimentwise error. Additionally, SEM allows for the evaluation of latent variables, which allows for the examination of the constructs while controlling for measurement error, whereas other statistical techniques assume zero measurement error which misrepresents reality. So, results from this study strengthen the existing literature through the use of a stronger statistical method than has been traditionally employed.

\section{Definition of Terms}

Anxiety: The experience of the following symptoms: dry mouth, rapid breathing in the absence of physical exertion, trembling, panic, worry about panic, feeling scared without any good reason, and changes in heart action in the absence of physical exertion (Lovibond \& Lovibond, 1995).

Binge eating: An individual eating what other people would regard as an unusually large amount of food (given the circumstances) while having a sense of having lost control over his/her eating (at the time that he/she was eating) (Fairburn \& Beglin, 2008). 
Depression: The experience of the following symptoms: lack of positive feelings, lack of initiative, lack of anything to look forward to, lack of enthusiasm, feeling downhearted, feeling worthless, and feeling that life is meaningless (Lovibond \& Lovibond, 1995).

Distress tolerance: One's perceived ability to withstand negative emotional experiences (Simons \& Gaher, 2005; Zvolensky et al., 2011).

Emotional reactivity: The extent to which an individual experiences the intensity, sensitivity, and persistence of emotions (Nock, Wedig, Holmberg, \& Hooley, 2008).

Fasting: An individual going for a long period of time (8 waking hours or more) without eating anything at all in order to influence his/her weight or shape (Fairburn \& Beglin, 2008).

Learned helplessness: The extent to which an individual believes that outcomes are independent of his/her behavior (Eisenberger, Park, \& Frank, 1976).

Limiting: An individual deliberately trying to limit the amount of food he/she eats in order to maintain or achieve a significantly low body weight (a weight that is less than minimally recommended) (Fairburn \& Beglin, 2008).

Nonsuicidal self-injury: An individual purposely hurting him/herself (e.g., cutting or burning) without wanting to die (Nock, Holmberg, Photos, \& Michel, 2007).

Purging: An individual making him/herself sick (vomit) as a means of controlling his/her weight or shape (Fairburn \& Beglin, 2008).

Suicidal ideation: An individual having thoughts of killing him/herself (Nock et al., 2007). 
Suicide attempts: An individual having made an actual attempt to kill him/herself in which he/she had at least some intent to die (Nock et al., 2007).

\begin{abstract}
Assumptions
It was assumed that the level and frequency of the variables self-reported by the participants are reflective of the true level and frequency of the variables experienced by the participant. It was also understood that the reported lifetime frequencies of selfharming behaviors may not be exactly accurate given the impact of time on memory. However, it was assumed that the recalled frequency is still representative of the overall frequency with which the individual has engaged in self-harming behaviors. For instance, it was understood that an individual who reports 10 episodes of NSSI still represents less severity of the behavior than another individual who reports 100 episodes of NSSI, even if the individual truly experienced 12 episodes of NSSI.

It was assumed that self-reported DT is the form of DT most relevant to clinical settings because subjective data is most easily and cheaply obtained by a clinician in most clinical practice settings. It was assumed that differences in the way self-reported DT and behaviorally-assessed DT relate to outcome behaviors in the extant literature is due to differences between the constructs being measured rather than self-report or behavioralassessment being poor means of assessing DT.

It was assumed that the self-damaging behaviors assessed in this study are, in fact, harmful to the individual who engages in them. Thus, lower frequency of the selfdamaging behaviors was assumed to be less damaging than greater frequency of the selfdamaging behaviors. It was understood that engagement in some of the self-damaging behaviors (e.g., NSSI) may provide temporary respite from the negative emotions
\end{abstract}


associated with severe and persistent trauma (e.g., sexual abuse). However, it was assumed that the self-damaging behaviors, if sustained, will result in greater long-term harm to the individual. In other words, none of the self-damaging behaviors examined in this study were considered socially acceptable or healthy forms of coping.

It was assumed that the self-damaging behaviors assessed in this study are representative of larger patterns of harmful behaviors. For instance, it was assumed that a history of a suicide attempt is representative of a broader pattern of mood disruption and suicidal ideation. In other words, the self-damaging behaviors were not an isolated occurrence (e.g., in response to a dare, to fulfill a political or religious objective), but rather most likely occurred in conjunction with other disruptive thinking and behavior patterns. However, it was understood that engagement in the self-damaging behaviors alone may not meet criteria for a diagnostic category.

I understand that I entered into this study with anecdotal evidence and theory, based on my clinical experience. Yet, I was open to finding whatever the data revealed and was not committed to or invested in the data working out in a specific way. To this end, I conducted a broad literature review and was open to the modification of the models to best fit the data.

\section{General Methodology}

This study employed a nonexperimental research design. A nonexperimental design was appropriate for my study since I desired to measure the variables as they are experienced in the respondents' daily contexts rather than in an experimental environment. My data was gathered using survey research. Survey research was appropriate for my study since the variables of interest were subjective. 
The sample for this study consisted of adults, 18 years or older, who resided in the United States of America. Screening questions ensured that the participants were 18 years or older and that the participants had experienced at least one of the self-harming behaviors over the course of their lifetime. The sample was collected using convenience sampling. The sample was drawn from respondents who were signed up to complete surveys on QuestionPro (QuestionPro, 2016), which is an online platform that helps researchers distribute surveys to target populations.

All variables were quantified by participant self-report. Demographic variables were self-selected on multiple-choice items. Anxiety, depression, emotional reactivity, and learned helplessness were quantified through self-report on Likert-scale items. Twoweek and lifetime frequencies of self-damaging behaviors were quantified by quantitative free-response.

I analyzed my data using SEM in order to determine the strength of the relationship between the variables. Structural equation modeling was a good fit for my study since it allowed me to examine models developed based on theory. It also allowed me to examine multiple independent and dependent variables simultaneously. Rather than a strictly confirmatory approach, I shifted to a model-generating approach to model analysis (Byrne, 2010) when the initial models were not a good fit for the data and the initial models were respecified based on the data. The goal was to find models which were both theoretically meaningful and well-fit to the data.

\section{Limitations}

Several limitations should be considered with this study. Most notably, my study utilized a nonexperimental research design. As such, my results speak to correlation and 
prediction but not causation. However, a nonexperimental design was appropriate for my study since I desired to measure the variables as they are experienced in the respondents' daily contexts rather than in an experimental environment. Also, experimental manipulation of the variables of interest would have been unethical and dangerous given the nature of self-damaging behaviors.

The models tested were based upon the literature review outlined in the following chapter. The studies reviewed reflected greater representation of females than males and greater representation of Caucasians than other races. This may have been due to overrepresentation of females and Caucasians in the diagnoses (e.g., borderline personality disorder), behaviors (e.g., eating disorders), or samples (e.g., clinical populations) of interest. While my survey was distributed to a representative distribution on the basis of sex, females were also overrepresented in my respondents. This was either due to more females clicking on the link to my survey or due to more females endorsing a lifetime history of the self-damaging behaviors on the screener question. Additionally, although representation of minority ethnicities in my respondents exceeded representation of minority ethnicities in the general online population, the majority of my respondents were Caucasian. Therefore, it is understood that my hypothesized models were drawn from literature which was not equally representative and my final models were revised based on respondents who were not equally representative. So, my results most fully describe females and Caucasians and will need to be replicated in different populations before results are generalized to other populations.

Also, all variables were assessed using self-report measures. This may have resulted in participants answering surveys in ways which were socially desirable, rather 
than in ways which accurately reflect their true behavior. However, the subjective nature of my variables required self-report. Given the sensitive nature of the variables of interest in this study, anonymous surveys may have allowed for more open sharing of risky behaviors than other data collection methods would have facilitated (Palmgreen et al., 2002, as cited in Beck, Daughters, \& Ali, 2013). Additionally, my research was intended to be applicable in clinical work in which a clinician's data is primarily client self-report through narrative or brief assessments.

Further, since my survey was hosted online, it limited my sample to individuals who had internet access, were comfortable with the use of the internet, and who had signed up specifically for completing online surveys through QuestionPro. It may be that individuals who voluntarily sign up to complete online surveys differ from the general population in their levels of the variables of interest. However, the online platform allowed for data collection from respondents who are heterogeneous in age, ethnicity, geographic location, socioeconomic status, and sex. Additionally, the vast majority of research on DT has been conducted with undergraduate or clinical populations, so it was important to survey a nonclinical population in order to broaden the literature.

Additionally, I analyzed my data using SEM. So, my results speak to the degree of fit observed between my resulting SEM models and the current sample. It is possible that different models (including different variables or the same variables arranged in a different configuration) would be a better fit for the data. The final models may not include all contributing variables, given the complexity of human behavior. Rather, the key contributing variables were drawn from the extant theory and included in the hypothesized models. Structural equation modeling was a good analysis approach for my 
research questions since it allowed for the assessment of multiple latent variables simultaneously. Other limitations which emerged in the data analysis process (e.g., missing values, outliers, skewed items), are discussed in chapter 5 since they are best understood in the context of results.

\section{Delimitations}

This study had five delimitations. First, the surveys were completed through an online research platform, QuestionPro. So, all respondents had to be part of QuestionPro's respondent panel. This implied computer literacy and internet access. Second, the surveys were distributed only to respondents who live within the United States of America. Third, only individuals at least the age of 18 were allowed to participate. Fourth, individuals had to endorse a lifetime history of at least one of the selfdamaging behaviors of interest in this study. Fifth, the sample size was limited to 300 participants due to financial and time constraints on the data collection process.

\section{Summary}

In this chapter, the background of the problem was outlined and the present study was introduced. The present study was designed to expand understanding of individual difference factors in DT. Specifically, the contributions of emotional reactivity and learned helplessness were examined to determine if they are related to an individual's level of DT. Additionally, this study examined the relationship between DT and several self-damaging behaviors: fasting, restricting, binging, purging, NSSI, suicidal ideation, and suicide attempts. This research was important because our currently limited knowledge of the factors accounting for individual differences in DT has hindered the 
development and refinement of prevention programs and interventions for individuals with low DT. This lack of knowledge needed to be rectified since low DT is associated with self-damaging behaviors which are related to a host of negative outcomes and diminished positive outcomes.

The hypotheses and research questions were defined in this chapter. The conceptual framework and assumptions of the study were also presented. Limitations and delimitations were mentioned and the key terms defined.

This dissertation includes four more chapters. Chapter 2 consists of a detailed literature review. General empirical and theoretical research related to DT is examined. Chapter 2 also presents the history and definition of the concepts of emotional reactivity, learned helplessness, and DT. Then, chapter 2 outlines what is known and unknown about the relationship between emotional reactivity and DT and the relationship between learned helplessness and DT. Lastly, it describes what is known and unknown about the relationships between DT and the self-damaging behaviors.

Chapter 3 discusses the methodology used to test the hypotheses. Chapter 3 outlines the procedures used to obtain participants, measure the data related to the hypotheses, and analyze the data. It also describes the means by which participants were informed of the potential risks and benefits of the study and how their consent to participate was obtained.

Chapter 4 presents the results of the research. It describes the sample and variables. It also describes reliability of the instruments and correlations between the variables. Then, it presents the results of model testing and modifications made to the hypothesized models. 
Chapter 5 discusses the implications of the research based on the research questions and extant literature. It outlines implications of the results for clinical intervention and for prevention practices. It discusses contributions to the existing literature and recommends areas for further study. 


\section{CHAPTER 2}

\section{LITERATURE REVIEW}

\section{Background to the Problem}

Distress tolerance is a concept with importance across multiple diagnostic categories (Bardeen et al., 2013; Kiselica et al., 2014). In particular, low distress tolerance has been linked to behaviors that often immediately reduce the individual's distress, but which cause psychological and often physical harm by avoiding real-life issues or problems (McHugh et al., 2014). These behaviors which have been linked with low distress tolerance include EDs, NSSI, and suicidality (Anestis, Pennings, et al., 2013; Anestis et al., 2007). These symptoms are important for study since these symptoms cause physical harm to individuals engaging in them and also since individuals with these self-damaging behaviors are frequent consumers of clinical services.

Moreoever, distress tolerance is thought to be malleable in response to clinical intervention (Marshall et al., 2008). As such, distress tolerance skills training is incorporated in a variety of therapeutic approaches including CBT, DBT, ACT, and DTspecific approaches. However, factors accounting for individual differences in level of distress tolerance remain mostly unidentified (G. Feldman et al., 2014). This "absence of knowledge about factors that account for individual differences in DT hinders efforts to target and increase DT" (Marshall et al., 2008, p. 2). 
In this study, I tested two models (a two-week model and a lifetime model) of individual difference factors in emotional distress tolerance in the context of selfdamaging behaviors (fasting, restricting, binging, purging, NSSI, suicidal ideation, and suicide attempts). My goal was to learn about factors influencing distress tolerance in hopes that this knowledge will better inform and refine distress tolerance intervention efforts. Learning about contributing factors may also inform the development of prevention programs aimed at building distress tolerance before clinical symptoms develop. Thus, in this literature review I investigate what is known and unknown about two potential individual difference factors in emotional distress tolerance-emotional reactivity and learned helplessness - in the context of fasting, restricting, binging, purging, NSSI, suicidal ideation, and suicide attempts. For, further knowledge of DT's development may deepen our understanding of the etiology of self-damaging behaviors as well as our understanding, and thus the effectiveness, of interventions for these behaviors (Cummings et al., 2013, p. 735).

\section{Distress Tolerance Introduction}

In this section, I will first define both broad distress tolerance and narrower emotional DT. I will then describe the criteria I used to identify studies for inclusion in this literature review. I will also provide an overview of the instruments which are used to measure emotional DT. Lastly, I will report on the factor structure of broad distress tolerance. 


\section{Definition and Terminology of DT}

The term distress tolerance has been used to refer to a broad construct of experiential tolerance but also to a more narrow type of emotional tolerance. Broadly speaking, "distress tolerance involves individual differences in the capacity to tolerate aversive internal states (e.g. pain, negative emotions, and body sensations) (Weems, 2011, p. 28). Within broad distress tolerance, narrower constructs of tolerance for specific types of distress have been proposed and researched. These narrower constructs include tolerance of ambiguity (TA), intolerance of uncertainty (IU), discomfort intolerance (DI), distress tolerance for negative emotional states (DT), frustration tolerance (FT), physical tolerance, and cognitive tolerance. For a summary of the conceptual differences among these constructs, see Zvolensky et al. (2011).

The focus of this study is on the narrow construct of tolerance of negative emotion. When Simons and Gaher (2005) developed the Distress Tolerance Scale to measure tolerance of negative emotion, they used the same term, distress tolerance, to describe emotional distress tolerance as others had used to describe broader experiential distress tolerance. This same term is used in the extant literature when referring specifically to emotional distress tolerance. Since this specific facet of tolerance is the

focus of this study, I use the term distress tolerance (and likewise the abbreviation DT) to refer to emotional distress tolerance as conceptualized by Simons and Gaher. I specify when I use the term in a broader sense. Simons and Gaher (2005) defined emotional DT as "the ability to experience and withstand negative psychological states" (p. 83). Zvolensky et al. (2011) defined DT as “one's perceived ability to tolerate emotional 
distress" (pp. 13-14). Drawing from these definitions, I defined DT as one's perceived ability to withstand negative emotional states.

\section{Inclusion in Literature Review}

In an effort to study the relationships among the narrow factors, many studies incorporate measures of more than one aspect of general distress tolerance. Since the focus of this review is on emotional DT, I incorporated studies measuring other aspects of general distress tolerance only when they were studied in addition to emotional DT. Also, studies of emotional DT sometimes use self-report measures and other times use behavioral measures of emotional DT. Self-report measures of emotional DT ask the participants to answer questions about their experience of psychological distress. Behavioral measures of emotional DT typically involve having the participant engage in a task (such as attempting unsolvable math problems) that is meant to produce psychological distress (such as frustration) and then defining DT as latency to termination on the task. The focus of my study was on self-perceived DT, since it is most readily assessed in clinical work. So, I included studies using behavioral measures of DT only when (a) they were utilized in conjunction with self-report measures of DT or (b) when results with behavioral measures opposed results for self-report measures.

When behavioral measures are included, it is important to note that task persistence and emotional DT are not synonymous concepts. For example, an individual who enjoys doing a task (e.g., solving math problems) is expected to persist longer at the task than an individual who does not enjoy the task. This does not indicate greater emotional DT for the individual who persists longer since emotional DT involves both subjective emotional distress and task persistence (Lynch \& Mizon, 2011). Additionally, 
the type of tasks incorporated in behavioral measures of DT may elicit a different type or different degree of distress than non-laboratory stressors. Especially since, in a laboratory setting, the participant is aware that they can stop the distressing task at any time (which is often not the case with real-world stressors). So, results from behavioral measures should be interpreted carefully, since they may not represent how an individual deals with real-world distress.

Further, studies of emotional DT sometimes include measures of physical distress tolerance through behavioral measures or self-report measures. Behavioral measures of physical distress tolerance usually involve having the participant engage in a physically strenuous task (such as holding their hand in cold water) and then defining physical distress tolerance as latency to termination on the task (i.e., taking their hand out of the cold water). Self-report measures of physical distress tolerance ask the participants to complete questions regarding their experience of physical distress. I included studies using behavioral and self-report measures of physical distress tolerance only when (a) they were utilized in conjunction with self-report measures of emotional DT or (b) when a study of the relationship of interest using emotional DT was not evident in the extant literature.

\section{Measures of DT Overview}

For a comprehensive review of instruments available for the measurement of DT, see Zvolensky et al. (2011). Typical instruments used to assess DT are introduced as they occur in the narrative of this review. However, one clarification ought to be offered at the outset. Two different self-report DT instruments have the same name: Distress Tolerance Scale. For the sake of clarity, the Distress Tolerance Scale as developed by Simons and 
Gaher (2005) is referred to as the sDTS throughout this review. The Distress Tolerance Scale as developed by Corstorphine, Mountford, Tomlinson, Waller, and Meyer (2007) is referred to as the cDTS throughout this review.

\section{Factor Structure of Broad Distress Tolerance}

Several models have been proposed to explain the relations between various aspects of broad distress tolerance. These models have originated either theoretically or as the result of factor analysis. McHugh and Otto (2012) used exploratory $(N=200,77 \%$ female, $84 \%$ Caucasian, mean age $=36$, nonclinical sample) and confirmatory factor analysis (CFA; $N=100,70 \%$ female, $79 \%$ Caucasian, mean age $=36$, clinical sample) to examine the latent structural relationship among anxiety sensitivity (AS), FT, DI, and DT. They analyzed the latent structure of four measures in their review, with the sDTS as their measure of emotional DT. Their results suggested that these aspects are best conceptualized as a single higher-order factor of distress intolerance (Nonclinical: $\chi^{2}(49)$ $=85.86, \mathrm{SRMR}=.05, \mathrm{RMSEA}=.06, \mathrm{TLI}=0.96, \mathrm{CFI}=.97 ;$ Clinical: $\chi^{2}(49)=72.59$, SRMR $=.06, \mathrm{RMSEA}=.07, \mathrm{TLI}=0.95, \mathrm{CFI}=.97)$. However, a single-factor model of broad distress tolerance has been questioned in subsequent research.

For instance, Bardeen et al. (2013) conducted CFA on the latent factor structure of broad distress tolerance. They tested the model developed by Zvolensky, Vujanovic, Bernstein, and Leyro (2010) which proposed one higher-order broad experiential distress factor and five lower-order factors: tolerance of uncertainty (the inverse of IU), TA, FT, DI and DT. Bardeen et al. (2013) analyzed eight measures of distress tolerance in their review, with the sDTS as their measure of emotional DT. In a nonclinical sample $(N=$ $830,61 \%$ female, $81 \%$ Caucasian, mean age $=34)$, the proposed model was supported $\left(\chi^{2}\right.$ 
$=230.95, p<.001, \mathrm{RMSEA}=.077, \mathrm{CFI}=.96, \mathrm{NNFI}=0.95)$. Results indicated that tolerance of uncertainty, TA, DT, DI, and DT are distinct factors and represent unique aspects of broad distress tolerance.

Bebane, Flowe, and Maltby (2015) conducted exploratory factor analysis (EFA) of the latent factor structure of broad distress tolerance. They also examined the fivefactor model which was proposed by Zvolensky et al. (2010) and supported by Bardeen et al. (2013). Bebane et al. (2015) analyzed five measures of distress tolerance in their review (all of which were included in Bardeen et al.'s study), with the sDTS as their measure of emotional DT. In an undergraduate student sample ( $N=511,84 \%$ female, $61 \%$ Caucasian, mean age $=20$ ), EFA supported the proposed model (all factor loadings on the retained factors were >.55). Bebane et al. (2015) then compiled a shorter scale which consisted of four items drawn from each of the five scales. The resulting 20 -item scale was administered to a nonclinical sample $(N=157,71 \%$ female, $73 \%$ Caucasian, mean age $=28)$ and results of CFA upheld the five-factor model $\left(\chi^{2}=272.874, p<.001\right.$, RMSEA $=.067$, SRMR $=.061, \mathrm{CFI}=.916, \mathrm{NNFI}=0.900)$.

M. Mitchell, Riccardi, Keough, Timpano, and Schmidt (2013) used CFA to examine the latent structural relationship between DT, DI, and AS. They analyzed three different distress tolerance measures, with the sDTS as their measure of emotional DT. They tested three different proposed models of the relationship. The first model proposed that DT, DI, and AS are all lower-order factors of one higher-order latent variable representing overall tolerance of distress. The second model was that of Bernstein, Zvolensky, Vujanovic, and Moos (2009) which proposed that there are two higher-order latent variables. The first higher-order variable would represent tolerance of emotional 
distress, and AS and DT would both be lower-order factors. The second, higher-order latent variable would represent tolerance of physical distress, with DI as a lower-order factor. The third model was that of Schmidt, Mitchell, Keough, and Riccardi (2011) which proposed a similar structure to the second model, except that AS is a subcomponent of DT.

M. Mitchell et al. (2013) drew their sample from both undergraduate students $(N$ $=411,71 \%$ female, $70 \%$ Caucasian, mean age $=19)$ and outpatients with anxiety disorders $(N=253,70 \%$ female, $73 \%$ Caucasian, mean age $=33)$. DT was assessed through self-report via the sDTS. In the undergraduate sample, the third model (which proposed emotional distress as a higher-order factor with DT as a lower-order factor and AS as a subcomponent of DT and physical distress as a higher-order factor with DI as a lower-order factor) was the best fit, providing a moderate fit to the data $\left(\chi^{2}=2.70, p=\right.$ .10$, RMSEA $=.06, \mathrm{CFI}=.98, \mathrm{TLI}=0.91)$. In the outpatient sample, the third model was again the best fit, providing a good fit to the data $\left(\chi^{2}=0.61, p=.43\right.$, RMSEA $=.06$, CFI $=1.00, \mathrm{TLI}=1.04)$. In both samples, models 1 and 2 were both poor fits for the data. These results suggest that DT involves tolerating many different negative emotional states, one of which is tolerance of anxiety (M. Mitchell et al., 2013).

Thus, the two multifactor models vary in the relationship between emotional distress and physical distress. The model by Schmidt et al. (2011) which was supported by M. Mitchell et al. (2013) holds that emotional distress tolerance and physical distress tolerance are both distinct higher-order factors. Whereas, the model by Zvolensky et al. (2010), which was supported by Bardeen et al. (2013) and Bebane et al. (2015), holds that both physical distress tolerance and emotional distress tolerance are lower-order 
factors to one higher-order experiential distress tolerance. However, both models suggest that the single-factor model proposed by McHugh and Otto (2012) may not fully represent the concept of broad distress tolerance. This may be due to McHugh and Otto (2012) using only four instruments in their EFA. As such, results of studies utilizing their instrument, the Distress Intolerance Index (DII), were cautiously interpreted.

\section{Theoretical Framework}

Now that I have outlined the relationship between broad distress tolerance and emotional DT, I will describe my theoretical framework of individual difference factors in emotional DT. First, I will discuss the influence of Linehan's theory of emotion dysregulation. Second, I will discuss the influence of Lynch and Mizon's theory of distress tolerance. I conclude by discussing the resulting models which I examined in this study.

\section{Linehan's BioSocial Theory of Emotion Dysregulation}

The concept of DT has been popularized by DBT, as developed by Linehan. Initially writing in 1993, Linehan (2015) developed a biosocial theory of emotional and behavioral dysregulation. She conceptualized from a third-wave behavioral perspective. This perspective views cognitions as behaviors and thus values both behavior and cognitions as important determinants in emotions and actions. This perspective also emphasizes examination and alteration of the function of behaviors (Lynch \& Mizon, 2011).

The model Linehan (2015) developed was largely based on individuals with BPD, but has since been extended to other populations. Linehan conceptualized emotion 
regulation as an individual's ability to modulate emotional experiences and responses. For instance, the ability to attend to mood-incongruent goals even while experiencing heightened emotions. She held that emotion regulation "can be automatic as well as consciously controlled" (p. 6). The aim of DBT is to teach participants skills for consciously regulating their emotional responses so that these skills might then be rehearsed to the point of automaticity.

The first half of her theory suggested that poor emotion regulation results from biological vulnerabilities to "negative affectivity, high sensitivity to emotion cues, and impulsivity" (p. 7). These biological vulnerabilities may be the result of genetics, intrauterine factors, or health factors influencing brain development in childhood. Emotional vulnerability is defined as "(1) very high negative affectivity as a baseline, (2) sensitivity to emotional stimuli, (3) intense response to emotional stimuli, and (4) slow return to emotional baseline once emotional arousal has occurred" (p. 6). So, individuals with high emotional vulnerability experience emotions more frequently, more intensely, and for longer periods of time than individuals who are not emotionally vulnerable. Individuals with high impulsivity have more difficulty inhibiting unwanted behaviors and organizing goal-oriented behaviors than individuals who are not impulsive.

The second half of the model developed by Linehan (2015) proposed that emotional and behavioral dysregulation results from an invalidating or ineffective caregiving environment in childhood. This type of environment is characterized by frequent invalidation of emotions, poor modelling of emotional expression, a child-parent interaction style which heightens emotional arousal, and a parenting style which does not match the child's temperament. Linehan thus proposed that the social environment and 
biological vulnerabilities interact to result in emotional and behavioral dysregulation. For instance, an invalidating environment may be especially harmful for a child with high emotional vulnerability.

\section{Linehan's Model of DT Skills Training}

In response to her model of emotional and behavioral dysregulation, Linehan (2015) developed a dialectical therapeutic approach which includes four modules of skills training. One of the dialectics incorporated in her model is that clients must balance accepting themselves as they currently are with needing to make personal changes. Reflecting this balance, she included two skill modules aimed at increasing acceptance skills (mindfulness skills and DT skills) and two skill modules for increasing change skills (interpersonal effectiveness skills and emotion regulation skills).

In this framework, Linehan (2015) defined DT as the ability to "perceive one's environment without putting demands on it to be different; to experience one's current emotional state without attempting to change it; and to observe one's own thoughts and action patterns without attempting to stop or control them" (p. 416). DT skills are included as one of the skills training modules to address dysregulated behaviors which are often present in individuals with emotional dysregulation. For, these behaviors are viewed as ineffective responses to problems which are utilized when the individual cannot withstand emotional distress long enough to seek a more effective response. Thus, the ability to tolerate distress is viewed as important because distress is both an inevitable part of life and also an inevitable part of the change process. Trying to avoid or remove distress may itself lead to more distress or inhibit the change process. The skills taught in 
this DT module focus on distracting, self-soothing, accepting reality, remaining mindful (attending to the present moment), and refraining from impulsive action.

\section{Lynch and Mizon's Three-Factor Model of DT}

Lynch and Mizon (2011) also approached the concept of DT from a third-wave behavioral perspective. As such, they viewed distress intolerant behavior as an operant response which is "reinforced by the short-term benefits of relief from aversive tension, despite potential long-term negative consequences" (p. 62). In addition to low DT resulting in psychopathology, they proposed that overly high DT (which they term distress overtolerance) may also result in psychopathology. They proposed a three-factor model of factors influencing the development of distress intolerant and overtolerant behavior: (a) biological predispositions (b) sociobiographic feedback, and (c) situational factors.

Firstly, the authors suggested that biological vulnerabilities may influence distress intolerant or overtolerant behavior. Similarly to Linehan (2015), they suggested that distress intolerant behavior may be influenced by genetic vulnerabilities for greater emotional pain sensitivity, greater emotional pain reactivity, greater impulsivity, greater novelty seeking, greater harm avoidance, lower physical pain sensitivity, and lower reward dependence. For these suggestions, they referenced literature supporting these trends in individuals with BPD who are, by definition, likely to engage in distress intolerant behaviors such as NSSI.

Secondly, the authors suggested that sociobiographic feedback may influence distress intolerant or overtolerant behavior. Similarly to Linehan (2015), they suggested that distress intolerant behavior may be influenced by a childhood environment in which 
emotional experiences were typically invalidated through contradiction, neglect, or punishment, but intense emotional reactions were occasionally reinforced through supportive attention. As such, the child would not learn to respond to distress with appropriate awareness and judgment. The authors drew from literature supporting links between emotionally invalidating childhood environments and intense negative emotions, chronic emotional inhibition, and BPD. Of note, the authors seem to emphasize sociobiographic feedback as historic influences, such as childhood environment, on an individual's current functioning. However, the proposed model is reciprocal, which recognizes that an individual's history is continuously being written.

Lastly, the authors suggested several situational factors which may influence distress intolerant or overtolerant behavior. Firstly, establishing operations are contextual factors which temporarily make stimuli more likely to elicit aversive tension. For illustration, they suggested that for an individual with an ED, the recent act of an unsatisfactory weighing experience may serve as an establishing operation which makes food-related stimuli temporarily more likely to elicit aversive tension and engagement in distress intolerant or overtolerant behavior. Secondly, cue factors refer to conditioned links between stimuli and context. These cue factors can include unconditioned stimuli, classically-conditioned stimuli, and operant-conditioned stimuli. As an example of an operant-conditioned stimuli, they spoke of a child associating a parents' mood with greater or less likelihood for reinforcement of their distress intolerant or overtolerant behaviors. Lastly, reinforcing factors are internal and external environmental factors which reinforce an individual's actions. For example, these reinforcing factors might include lessened aversive tension or increased social approval. Of note, the authors seem 
to distinguish these situational factors as more immediate than sociobiographic feedback. In illustration, an individual who binge eats in response to a negative emotional state may be influenced by a childhood environment that praised him/her for eating in response to strong emotions (sociobiographic feedback) and/or by the smell of enticing food in the immediate environment (situational factors).

\section{Lynch and Mizon's Proposed Individual Difference Factors in DT}

Lynch and Mizon (2011) predicted that "individual differences in learned industriousness (which will affect task persistence) and emotional reactivity (which will affect subjective distress) will be key determinants of the emergence" of distress overtolerant and distress intolerant behavior (p. 60). Firstly, they proposed that high emotional reactivity is a predictive factor for both distress intolerance and distress overtolerance. For, an individual's degree of emotional reactivity influences the amount of distress an individual will experience and it can thus "accentuate any tendencies toward maladaptive coping" (Lynch \& Mizon, 2011, p. 60). Secondly, they proposed that high emotional reactivity combines with either a high level of learned industriousness to produce distress overtolerant behavior or a low level of learned industriousness to produce distress intolerant behavior. If an individual has a learning history in which he/she was rewarded for low effort, avoidance, or escape responses (low learned industriousness), he/she will be unlikely to persist in tolerating distress, which requires high effort. Whereas, if an individual has a learning history in which he/she was rewarded for high effort responses (high learned industriousness), he/she will be likely to persist in the effort required to tolerate distress. 
Thus, Lynch and Mizon (2011) proposed that DT level results from the interaction between the influences of nature (biological vulnerabilities) and nurture (sociobiographic feedback and situational factors). In keeping with the behavioral nature of their model, neither of these influences account for agency on the part of the individual. Although the authors view distress intolerant and distress overtolerant behaviors as automatic and occurring without conscious control, they also make statements that suggest even they believe human agency is involved in one's responses to distress. For instance, the authors view a healthy level of DT as requiring "conscious awareness of internal states" as well as the "ability to not automatically and without awareness respond to aversive tension with a change strategy" (such as in distress intolerance) and the ability not to "habitually ignore warning signs of distress" (such as in distress overtolerance) (Lynch \& Mizon, 2011, p. 71). Additionally, when it comes to changing an individual's level of DT the authors suggested that interventions which aim to increase mindfulness and the ability to observe, but not always react, to aversive tension might be beneficial.

\section{My Theoretical Model of Individual Difference Factors in DT}

As both Linehan (2015) and Lynch and Mizon (2011) proposed, I also hypothesized that emotional reactivity is an individual difference factor in DT. That is, I hypothesized that a high level of emotional reactivity would be associated with low DT. As Lynch and Mizon (2011) proposed, I hypothesized that learned industriousness would also be an individual difference factor in DT. That is, low learned industriousness (measured in this study as high learned helplessness; see discussion below) would be 
associated with low DT. Whereas Lynch and Mizon spoke of learned helplessness as a nature factor, I conceptualized learned helplessness as an individual agency or effort factor. That is, an individual with high learned helplessness will expend low or no effort toward emotion regulation or problem-solving, while an individual with low learned helplessness will expend high effort toward emotion regulation or problem-solving. As such, I hypothesized that high emotional reactivity and high learned helplessness will contribute to low DT. I examined this hypothesized relationship specifically in the context of self-damaging behaviors.

Given the hypothesized theoretical model, I next examine literature regarding the factor structure of emotional DT and general research related to DT. Then, I look at literature regarding the role of emotional reactivity and learned helplessness in DT. Lastly, I look at the relationship between DT and covariates and the relationship between DT and self-damaging behaviors.

\section{Emotional DT Overview}

Factor Structure of Emotional DT

The factor structure of emotional DT has been studied through exploratory and confirmatory factor analysis. For instance, Simons and Gaher (2005) used exploratory and confirmatory factor analysis to examine the structure of DT, as measured by their instrument, the Distress Tolerance Scale. In their sample of undergraduate students $(N=$ $823,67 \%$ female, 94\% Caucasian, mean age $=20$ ), they identified a single higher-order DT factor with four lower-order factors $\left(\chi^{2}=517.39, p<.001, \mathrm{RMSEA}=.077, \mathrm{CFI}=\right.$ $.96, \mathrm{NNFI}=.96, \mathrm{SRMR}=.053)$. The lower-order factors include tolerance $(\mathrm{sDTS}$ : 
Tolerance), appraisal (sDTS: Appraisal), regulation (sDTS: Regulation), and absorption (sDTS; Absorption).

First, sDTS: Tolerance represents an individual "report[ing] distress as being unbearable" and being unable to "handle being distressed or upset" (Simons \& Gaher, 2005, p. 84). This factor was characterized by the item "I can't handle feeling distressed or upset” (p. 93). Second, sDTS: Appraisal represents an individual's "lack of acceptance of distress, being ashamed of being distressed, and perceiving one's coping abilities as inferior to others" (p. 84). This factor was characterized by the item "My feelings of distress or being upset scare me" (p. 93). Third, sDTS: Regulation represents an individual making "great efforts to avoid negative emotions and utilizing rapid means of alleviating the negative emotions" he/she experiences (p. 84). This factor was characterized by the item "I'll do anything to stop feeling distressed or upset" (p. 93). Fourth, sDTS: Absorption represents an individual reporting that his/her attention is consumed "by the presence of distressing emotions and that [his/her] functioning is significantly disrupted by the experience of negative emotions" (p. 84). This factor was characterized by the item "When I feel distressed or upset, I cannot help but concentrate on how bad the distress actually feels" (p. 93).

Factor analysis has also been conducted on DT through examination of the cDTS. Since this scale has largely been used in the study of EDs, the results of these analyses are included below in discussion of the relationship between DT and disordered eating behaviors. 


\section{General Research on Emotional DT}

Now that I have looked at the definition and factor structure of DT, I examine general research related to DT. In this section, I first review literature on the degree of stability in DT over time and the malleability of DT in response to clinical interventions. Then, I explore the relations between DT and two related concepts: avoidance and urgency. Lastly, I report on factors thought to influence DT including emotion regulation, attentional control, mindfulness, and personality traits.

\section{Stability of DT over Time}

Distress tolerance is thought to be a largely stable factor, unless targeted through intervention. (McHugh et al., 2014). Evidence for this conclusion comes from longitudinal studies of DT, as well as from intervention outcomes. First, I review literature that explores the longitudinal stability of DT examined at the aggregate and individual levels. Second, I examine studies which attempted to modify DT level by utilizing CBT approaches, DT-specific approaches, and other approaches. Lastly, I examine literature which does not support the malleability of DT.

Longitudinal study in early adolescents

Cummings et al. (2013) examined the stability of DT over the course of 4 years among early adolescents $(N=277,66 \%$ male, $48 \%$ Caucasian). The adolescents ranged in age from 9 to 13 at initial enrollment. DT was assessed using the Behavioral Indicator of Resiliency to Distress task (Lejuez, Daughters, Danielson, \& Ruggiero, 2006, as cited on p. 737) which is a behavioral measure of DT. The task was completed at year 1 (baseline), year 2, year 3, and year 4 . 
Evidence for the stability of DT was demonstrated at both the group and individual levels. In the sample as a whole, results indicated no significant change in mean-level DT across the four years $(F(3)=0.60, p=.61, d=0.04)$. On an individual level, rank order stability as evidenced by 4-year test-retest correlation $(r$ 's $=.25$ to .51 , $p$ 's $<.01$ ) was moderate and not clinically meaningful. Clinically significant (as indicated by the reliable change index $[\mathrm{RCI}]$ ) changes in individual DT levels over the four years occurred only minimally more than would be expected by chance alone, and an individuals' DT level was equally likely to increase as to decrease. The high mean-level stability, high individual-level stability, and moderate rank-order stability suggest that, without intervention, DT is relatively stable during early adolescence and reflects only the level of change typically observed in personality variables (Cummings et al., 2013). That some change occurred may suggest malleability due to developmental or environmental factors.

Longitudinal studies in adults

Kiselica et al. (2014) examined the stability of self-reported DT over the course of 6 months among undergraduate students $(N=233,73 \%$ female, 50\% Caucasian, mean age $=20$ ). Three measures of DT were utilized: the sDTS, the Frustration Discomfort Scale (FDS; Harrington, 2005, as cited on p. 248), and Tolerance of Negative Affective States Scale (TNASS; Bernstein \& Brantz, 2013, as cited on p. 248). The assessments were completed at baseline, at 3-month follow-up, and at 6-month follow-up.

Evidence for the stability of DT was demonstrated at the group and individual levels. In the sample as a whole, results indicated no significant change in mean-level DT across the time period and across all three measures of DT ( $\left.F^{\prime} \mathrm{s} \leq 2.00, p^{\prime} \mathrm{s} \geq .14\right)$. On an 
individual level, rank-order stability ranged from moderate (sDTS: males: $r$ 's $=.34$ to .45 , females: $r$ 's $=.33$ to .55$)$ (TNASS: males: $r$ 's $=.31$ to .53 ; females: $r$ 's $=.42$ to .56 ) to high (FDS: males: $r$ 's $=.71$ to .73 , females: $r$ 's $=.54$ to .67 ). This indicated that people change only moderately in their level of DT relative to their peers. This z-level of rank order variation was in keeping with that of other personality constructs. Further, the incidence of individual clinically significant changes in DT (determined by RCI) was not greater than that predicted by chance alone (sDTS: Time 1 to Time $2: \chi^{2}=.70, p=.706$, Time 2 to Time $\left.3: \chi^{2}=.43, p=.807\right)\left(\right.$ FDS: Time 1 to Time $2: \chi^{2}=.31, p=.857$, Time 2 to Time $\left.3: \chi^{2}=.19, p=.909\right)$ (TNASS: Time 1 to Time $2: \chi^{2}=.31, p=.857$, Time 2 to Time $\left.3: \chi^{2}=.34, p=.844\right)$. In addition, the incidence of clinically significant change was not correlated with age ( $r$ 's $\leq .11, p$ 's $\geq .124)$. As such, this study suggests that time does not change an individual's level of DT, regardless of the individual's age. The authors concluded that DT is a stable construct and that intervention may be needed in order to change DT level.

Simons and Gaher (2005) also examined the 6-month stability of DT when validating their instrument, the sDTS. Undergraduate participants $(N=823,67 \%$ female, 94\% Caucasian, mean age $=19$ ) completed the sDTS online at baseline and then at an average of 216 days later. Results indicated moderate test-retest reliability (ICC=.61). Macatee, Capron, Guthrie, Schmidt, and Cougle (2015) found identical test-retest reliability (ICC=.61) for the sDTS across a one-month interval among undergraduate students $(N=87,76 \%$ female, $67 \%$ Caucasian, mean age $=19)$. Thus, Macatee et al. (2015) supported the stability of DT, as measured by the sDTS over a 1-month time 
frame, and both Simons and Gaher (2005) and Kiselica et al. (2014) supported the stability over a 6-month time frame.

Evidence supporting modification through treatment

Modification through CBT. McHugh et al. (2014) examined the impact of an intensive, short-term CBT intervention on levels of DT, anxiety, and depression among a clinical sample participating in a partial hospitalization program. (In the study, the authors' spoke of the inverse of DT: distress intolerance. The terms have been reversed for continuity and clarity). Participants $(N=626,57 \%$ female, $91 \%$ Caucasian, mean age =34) completed measures of anxiety, depression, and DT (as measured by the DII) at pre- and post-treatment. The intervention consisted of five, 50-minute CBT skill groups a day for five days a week. Each participant also received two or three individual CBT counseling sessions each week. The average length of participation in the program was 8 days.

Results indicated that, on average, DT increased $(F(1,458)=94.27, p<.001$, partial $\left.\eta^{2}=.17\right)$ and anxiety $(t=-2.32, p<.001)$ and depression $(t=-22.62, p<.001)$ decreased from pre- to post-treatment. Specifically, $65 \%$ of the participants reported an increase in DT from pre- to post-treatment. Within this, $31 \%$ of the total participants evidenced a clinically significant (defined as an increase of 2 or more standard deviations from the pre-treatment mean) increase in DT. These participants who experienced a clinically significant increase in DT also reported less depression $(t(447)=4.39, p<.001$, $d=0.46)$ and anxiety $(t(166)=3.97, p<.001, d=0.69)$ at post-treatment than at pretreatment. Therefore, this study presented evidence that a short-term, targeted CBT 
intervention increased DT for more than half of participants while concurrently decreasing anxiety and depression symptoms.

Williams, Thompson, and Andrews (2013) studied the impact of six online CBT modules over a 10-week period on DT and depression (see below for a more detailed summary and sample statistics). They similarly found that DT (as measured by the sDTS) increased and depression severity decreased from pre-treatment to post-treatment among both outpatients and a nonclinical sample. Thus, both studies evidence the possibility of increasing DT through CBT interventions.

Modification through DT-specific intervention. Bornovalova, Gratz, Daughters, Hunt, and Lejuez (2012) examined the impact of three different intervention conditions on DT and depression symptoms among inpatients with substance use disorders (SUD). To do so, participants $(N=76,71 \%$ male, 90.5\% African American, mean age $=43$ ) were randomly assigned to one of three treatment conditions. First, participants in the treatment as usual condition received group counseling based on Alcoholics and Narcotics Anonymous curriculums. Second, participants in the supportive counseling condition received treatment as usual plus a non-specific supportive individual intervention. Third, participants in the Skill for Improving Distress Intolerance (SIDI) condition received treatment as usual plus a newly developed individual intervention drawn from DBT and ACT which specifically targeted DT through emotional exposure. Both the SIDI and supportive counseling conditions consisted of six, 90-minute, individual counseling sessions over a two-week period. To be included in the study, participants had to demonstrate low behaviorally-assessed DT. Distress tolerance was measured by the Computerized Mirror-tracing Persistence Task (MTPT-C, Strong et 
al., 2003) and the Computerized Paced Auditory Serial Addition Test (PASAT-C, Lejuez, Kahler, \& Brown, 2003).

Results indicated that the SIDI group evidenced greater increases in mean-level DT than the supportive counseling group $(F(1)=10.77, p<.001, d=1.00)$ and the treatment as usual group $(F(1)=3.99, p<.05, d=.60)$. This increase held after controlling for changes in depression symptoms. More participants in the SIDI group evidenced clinically significant increases in DT (as indicated by the RCI) than in the supportive counseling group $\left(\chi^{2}(2)=11.64, p<.01\right)$, but not than in the treatment as usual group $\left(\chi^{2}(2)=3.02, p<.22\right)$. In fact, the supportive counseling group evidenced a decrease in mean-level DT $(p<.05, d=-0.47)$.

Thus, this study suggested that DT can increase as a result of a brief, targeted treatment intervention. This study was strengthened by the use of two different comparison conditions and controlling for changes in depression symptoms. The study also ruled out differences in the therapeutic alliance between conditions $(F(1)=.33, p=$ $.57, d=17$ ). However, the sample size was relatively small so the results should be replicated with a larger sample.

The supportive counseling condition asked patients to express the distress they are experiencing, but did not offer education about building skills to handle their distress. Contrary to the intent of the intervention, participants in the supportive counseling condition experienced a decrease in DT. This may evidence the risk of decreasing DT with a non-targeted intervention. However, given the small sample size per condition, this result would need to be replicated in a larger sample. 
Evidence from other approaches. As detailed below, Booth, Keogh, Doyle, and Owens (2014) found that a group intervention derived from DBT resulted in concurrent increases in DT and decreases in NSSI up to three months post-treatment. Also, Norr, Allan, Macatee, Keough, and Schmidt (2014) found that a single, 50-minute, psychoeducational session focused on AS increased DT among undergraduate students with elevated anxiety.

Evidence opposing modification through treatment

Kapson, Leddy, and Haaga (2012) examined the impact of two different smoking cessation treatment groups on DT, functional responses (defined as plans for thoughts and actions indicative of positive coping skills) to negative thinking, and acceptance of negative automatic thoughts among a nonclinical sample desiring to quit smoking $(N=$ $101,51 \%$ female, $65 \%$ Caucasian, mean age $=43$ ). Functional responses to negative thinking were assessed using the Ways of Responding Test (Barber \& DeRubeis, 1992, as cited in Kapson et al., 2012, p. 1234) which asks participants to imagine an upsetting event and a corresponding negative thought and emotional reaction. The participants list their plans for managing the negative event, and raters categorize these plans as positive (functional), negative, or neutral responses. Both conditions consisted of eight, 90minute, group sessions over the course of 7 weeks. Though both conditions included psychoeducational information, the CBT condition addressed the topic of mood management whereas the comparison condition did not.

Overall, the CBT intervention was found to be effective in promoting smoking abstinence (defined as 7-day point prevalence abstinence as measured at 3 months post- 
treatment) among participants who had high levels of depression-proneness at baseline (35\%), but not among participants who had low levels of depression-proneness at baseline (10\%). The CBT intervention also increased functional responses to negative thinking, but only among participants who reported low levels of depression-proneness at baseline $\left(F(1,94)=4.83, p<.05\right.$, partial $\left.\eta^{2}=.05\right)$. Behaviorally-assessed DT (as measured by the PASAT-C and the MTPT-C) did not change from pre- to post-treatment $\left(F(1,94)=0.09, n s\right.$, partial $\left.\eta^{2}=.001\right)$.

This study found no changes in behaviorally-assessed DT as a result of a CBTbased intervention. However, the impact of the CBT-based intervention on smoking cessation was limited to individuals who reported high levels of depression-proneness at baseline. So, the effectiveness of this intervention is questionable both for impact on smoking cessation and on behaviorally-assessed DT.

\section{Relationship Between DT and Related Concepts}

DT has been theoretically and empirically related to several concepts. Among these concepts, avoidance and urgency are especially relevant in relation to maladaptive behaviors. So, in this section, I review research outlining the relationship between DT and avoidance and between DT and urgency.

Relationship between DT and avoidance

Theoretically, low DT is described as "an important motivator of maladaptive avoidance-based coping strategies” (McHugh, Reynolds, Leyro, \& Otto, 2013, p. 363). This was empirically tested by McHugh et al. (2013), who examined the role of DT and 
emotional regulation in avoidance. The study was conducted with both a nonclinical sample $(N=300,75 \%$ female, $82 \%$ Caucasian, mean age $=36)$ and an outpatient clinical sample receiving treatment for anxiety and depression $(N=100,62 \%$ female, $90 \%$ Caucasian, mean age $=31$ ). Distress tolerance was assessed through self-report via the DII (for the sake of consistency, scores have been reversed in this narrative). Results indicated that DT (nonclinical sample: $r=-0.67, p<.001$; clinical sample: $r=-0.69, p<$ .001) was negatively correlated with avoidance. Further, additional regression analyses revealed that DT and access to emotion regulation strategies were both independently predictive of avoidance in both samples (as indicated by a nonsignificant interaction effect, nonclinical: $\beta=-.05, t=-0.61, p=.54$, clinical: $\beta=-.04, t=-0.28, p=.78)$. In the nonclinical sample, DT $(\beta=.29, t=5.29, p<.001)$ and access to emotion regulation strategies $(\beta=.50, t=9.28, p<.001)$ explained $55 \%$ of the variance in avoidance. In the clinical sample, DT $(\beta=.47, t=5.81, p<.001)$ and access to emotion regulation strategies $(\beta=.40, t=4.86, p<.001)$ explained $62 \%$ of the variance in avoidance.

This study was strengthened by the inclusion of both a clinical and nonclinical sample. The similarity of the results across the two samples suggests the utility of these concepts for a range of functioning. However, the internal validity of the instrument assessing avoidance was limited. So, the study should be improved by using a more reliable measure of avoidance. If the results hold true, this study suggests that both limited DT and limited access to emotion regulation strategies contribute to avoidance and that it may be helpful to address both in interventions aimed at reducing avoidance behaviors (McHugh et al., 2013). 
Schloss and Haaga (2011) also examined the relationship between DT and experiential avoidance (defined as avoiding aversive internal experiences). The sample was composed of outpatient smokers $(N=100,51 \%$ female, $65 \%$ Caucasian, mean age $=$ 43). Distress tolerance was assessed behaviorally via the PASAT-C and MTPT-C. Results indicated that behaviorally-assessed DT was not correlated with avoidance (PASAT-C: $r$ 's $=-.06$ to $.16, n s$; MTPT-C: $r$ 's $=-.07$ to $.11, n s$ ). Similarly, the level of avoidance for individuals who persisted on the PASAT-C and MTPT-C until the tasks were completed did not differ from the level of avoidance for individuals who did not complete the tasks ( $p$ 's $>.2$ to $>.6$ ). Thus, it appears that behaviorally-assessed DT was related to avoidance in a different way than self-reported DT. Of note, this study compared behaviorally-assessed DT with self-reported experiential avoidance. Further research is needed to see if a behavioral measure of experiential avoidance (or a selfreport measure of DT) would yield a different pattern.

Relationship between DT and urgency

Weitzman, McHugh, and Otto (2011) examined the relationship between DT, access to emotion regulation strategies, AS, and urgency. Access to emotion regulation strategies is a factor of emotion regulation which refers to the extent to which individuals believe they can use strategies to regulate their upsetting emotions (Gratz \& Roemer, 2004) (for the sake of clarity, this will be referred to broadly as emotion regulation). Urgency was defined as "the propensity to act rashly in response to negative affect" (p. 1106). Distress tolerance was assessed through self-report via the sDTS. The study was conducted with both a nonclinical sample $(N=297,75 \%$ female, $83 \%$ Caucasian, mean 
age $=36)$ and an outpatient clinical sample receiving treatment for anxiety and depression $(N=99,65 \%$ female, $90 \%$ Caucasian, mean age $=31)$.

In the nonclinical sample, low DT $(B=.31, t=5.51, p<.01)$, but not AS $(B=$ $.001, t=0.33, p=.74)$, was associated with high urgency. The association between low emotion regulation and high urgency approached significance $(B=.12, t=1.90, p=.06)$. The full model predicted $31 \%$ of the variance in urgency $(F(3,293)=44.66, p<.001)$. In the clinical sample, low DT $(B=.28, t=3.04, p<.005)$ and low emotion regulation $(B=$ $.24, t=2.72, p<.01)$ were both associated with high urgency. Anxiety sensitivity was not a significant predictor $(B=.005, t=0.96, p=.34)$. Together, DT and emotion regulation predicted $44 \%$ of the variance in urgency $(F(3,95)=24.54, p<.001)$. A strength of this study was the use of both a clinical and nonclinical sample. However, both samples were largely female and Caucasian, so the generalization of results could be strengthened by replication of the study with a more diverse sample.

In summary, self-reported low DT has been found to be correlated with high avoidance in both outpatient and nonclinical samples. Distress tolerance was also found to be predictive of avoidance. Together, DT and emotion regulation explained just over half of the variance in avoidance. Whereas, behaviorally-assessed DT was not found to be correlated with avoidance in an outpatient sample. Low self-reported DT has also been found to be correlated with high urgency in both outpatient and nonclinical samples. Distress tolerance was also found to be predictive of urgency. Together, DT and emotion regulation explained just under half of the variance in urgency in the outpatient sample. Thus, it appears that DT is related to the concepts of avoidance and urgency. However, 
the literature suggests that DT is a factor predicting variance in avoidance and urgency, rather than avoidance and urgency being individual difference factors in DT.

\section{Factors Known to Influence DT}

Having clarified that the literature does not suggest avoidance and urgency as factors influencing DT, I will now examine literature supporting factors which are thought to influence DT. Each of the factors reviewed below has been examined as a factor influencing DT. Though these factors are not in the model I tested in this study, they provide important context as to what is already known about other potential individual difference factors in DT.

The influence of emotion regulation and attentional control

Though conceptually similar, emotion regulation and DT appear to be similar, yet distinct concepts (Brandt, Zvolensky, \& Bonn-Miller, 2013; McHugh et al., 2013; McHugh et al., 2014). As described above, McHugh et al. (2013) found strong correlation between DT and difficulties in accessing emotion regulation strategies in both a nonclinical $(r=-0.76, p<.001)$ and clinical sample $(r=-0.64, p<.001)$. Yet, DT and difficulties in accessing emotion regulation strategies each contributed to level of avoidance in a way that was additive, rather than interactive. In a study of depression and anxiety among individuals with HIV/AIDS, Brandt, Zvolensky, and Bonn-Miller (2013) found a self-report measure of DT (sDTS) and emotion dysregulation to share $33 \%$ of variance with one another $(r=-.58, p<.01)$. In a study of coping motives for alcohol use among trauma survivors, Vujanovic, Marshall-Berenz, and Zvolensky (2011) found emotional regulation and a self-report measure of (sDTS) to share $60 \%$ variance $(r=-.78$, 
$p<.01)$. Despite the shared variance, DT and emotion regulation each associated uniquely with coping motives for alcohol use (as indicated by squared semi-partial correlations).

This relationship is further attenuated by the mediating role of attentional control. Bardeen, Tull, Dixon-Gordon, Stevens, and Gratz (2015) examined the role of attentional control in the relationship between low DT and difficulties in accessing emotion regulation strategies (for the sake of clarity, this will be referred to broadly as emotion dysregulation). The sample was nonclinical $(N=93,63 \%$ female, 54\% Caucasian, mean age $=24$ ). Distress tolerance was assessed behaviorally through the PASAT-C, in which DT was defined as latency to termination.

Results indicated that emotion dysregulation interacted with attentional control in the prediction of behaviorally-assessed DT. Neither emotion dysregulation $(B=-2.06, \beta$ $=-0.11, p=0.34)$ nor attentional control $(B=-0.35, \beta=-0.12, p=0.27)$ had a direct effect on DT. However, the interaction of emotion dysregulation and attentional control was associated with DT $\left(\mathrm{B}=-0.07, \beta=-0.23, p=0.03, \Delta \mathrm{R}^{2}=0.05\right)$ : emotion dysregulation was negatively associated with DT, but only among individuals low in attentional control (not among individuals high in attentional control). Together, attentional control and emotion dysregulation explained $5 \%$ of the observed variance in DT. However, the small interaction effect $\left(f^{2}=.054\right)$ indicates continued unexplained variance in DT. Thus, emotional regulation may have a minor influencing role on behaviorally-assessed DT, but the influence is moderated by level of attentional control. 
The influence of mindfulness and rumination

G. Feldman et al. (2014) examined the role of mindfulness and rumination in DT among undergraduate females $(N=94,76 \%$ Caucasian, mean age $=21)$. Distress tolerance was assessed behaviorally through the MTPT-C. Results indicated that acting with awareness mindfulness (consciously concentrating on one's present experiences, $r=$ $.23, p<.05$ ), and reflection rumination (attempts to understand one's negative emotions, $r=.27, p<.01)$ were both positively correlated with DT. Additionally, acting with awareness mindfulness $(\beta=.23)$ and reflection rumination $(\beta=.28)$ together explained $14 \%$ of the variation in DT when accounting for task skill (the amount of time spent on the MTPT-C introductory tasks and the number of errors on the MTPT-C) and stress reactivity (change in negative affect over the course of the task). However, non-judging mindfulness $(r=.03, n s)$, non-reactivity mindfulness $(r=.11, n s)$, and brooding rumination $(r=-.02, n s)$ were not correlated with DT. Therefore, this study suggests that mindful awareness and reflective rumination are related to behaviorally-assessed DT, whereas other aspects of mindfulness and rumination are not.

Pearson, Lawless, Brown, and Bravo (2015) similarly examined the role of mindfulness in DT among undergraduate students $(N=994,64 \%$ female, $61 \%$ Caucasian, median age $=19$ ). Distress tolerance was assessed by self-report using the sDTS. The respondents were statistically classified into four groups: high mindfulness (high on observing, high on non-judging, high on acting with awareness), nonjudgmentally aware (low on observing, high on non-judging, high on acting with awareness), judgmentally observing (high on observing, low on non-judging, low on 
acting with awareness), and low mindfulness (low on observing, low on non-judging, low on acting with awareness).

Results indicated that the high mindfulness and non-judgmentally aware groups were statistically significantly highest in DT and lowest in depression symptoms, anxiety symptoms, and affective lability (see the original article for the many mean group values). Whereas, the low mindfulness group was statistically significantly lowest in DT and highest in depression symptoms, anxiety symptoms, and affective lability. The judgmentally observing group had statistically significantly less positive outcomes than the high mindfulness and non-judgmentally aware groups, but had statistically significantly more positive outcomes than the low mindfulness groups.

This study extended the findings of G. Feldman et al. (2014) by demonstrating that acting with awareness mindfulness is also associated with self-reported DT. Further, the Pearson study demonstrated that non-judgmental mindfulness is correlated with selfreported DT $(r=.52, p<.05)$, though it was not with behaviorally-assessed DT in the 2014 study. However, it must be noted that the Pearson study did not assess prediction, only correlation. So, directionality of the associations are unknown.

The influence of personality traits

Borderline traits. Bornovalova et al. (2008) found that self-reported difficulties in emotion regulation (as measured by the Difficulties in Emotion Regulation Scale, Gratz \& Roemer, 2004) and behaviorally-assessed DT (as measured by the PASAT-C and MTPT-C) were both predictive of BPD diagnosis. The sample was drawn from inpatients receiving treatment for SUDs $(N=76,67 \%$ male, $80 \%$ African American, mean age $=42)$. Together, difficulties in emotion regulation $(B=0.04$, Wald test $=4.45$, 
$p<.05)$, behaviorally-assessed DT $(B=-0.01$, Wald test $=9.07, p<.01)$, and covariates (gender, race, the presence of MDD, and the presence of bipolar disorder) reliably distinguished between participants with or without BPD $\left(\chi^{2}=41.16, p<.01\right)$ and accounted for $59 \%$ of the variance in BPD diagnosis status.

Building on this finding, Bornovalova, Matusiewicz, and Rojas (2011) examined the role of DT, negative emotionality, and negative affect intensity in BPD traits. The sample was drawn from members of the community who desired to quit smoking $(N=$ 110, 53\% male, $70 \%$ African American, mean age $=47$ ) as well as from inpatients receiving treatment for substance abuse $(N=76,67 \%$ male, $80 \%$ African American, mean age $=42$ ). The inpatient portion of the sample appears to be the same as the sample reported by Bornovalova et al. (2008). DT was assessed behaviorally through the PASAT-C and MTPT-C. Results indicated that negative emotionality $(r=-.05, n s)$ and affect intensity $(r=-.04, n s)$ were not correlated with DT. When affect intensity was controlled for in the model, there was not a significant main effect of DT on borderline traits $(\beta=-.04, n s)$. However, negative emotionality and DT interacted in the prediction of borderline traits $(\beta=-.25, p<.01)$ : individuals with high negative emotionality and low DT demonstrated the highest levels of borderline traits. This interaction only held true at low DT levels. When negative emotionality was controlled for in the model, there was a main effect of DT $(p<.05)$ in the prediction of borderline traits. There was also a significant interaction between DT and affect intensity $(\beta=-.21, p<.05)$ in the prediction of borderline traits: individuals with high affect intensity and low DT demonstrated the highest levels of borderline traits. This interaction was true at all levels of DT. As such, 
Negative emotionality places one at a risk for high levels of BPD, regardless of distress tolerance levels (although those with high levels of the former and low levels of [the latter] are most vulnerable). On the other hand, negative affect intensity is a risk factor for high BPD levels only in the context of low distress tolerance. (Bornovalova et al., 2008, p. 751)

Further, the interaction effects between negative emotionality and DT and between affect intensity and DT were found to only impact the self-harm symptoms cluster of borderline traits and the overall scale, not the identity problem, affective instability, or negative relationship symptom clusters. Therefore, individuals with low DT, high negative emotionality, and high affect intensity are at risk for engaging in selfharming behaviors. This study was strengthened by accounting for education level and number of SUD diagnoses as covariates.

Antisocial traits. Daughters, Sargeant, Bornovalova, Gratz, and Lejuez (2008) examined the role of DT in antisocial personality disorder (ASPD) among a sample of substance-dependent inpatient males $(N=127,88.2 \%$ African American, mean age $=$ 40). Distress tolerance was assessed behaviorally through the PASAT-C and MTPT-C. Despite similar self-reported post-task dysphoria $(F(1,126)=.02, p>.05)$, individuals with ASPD persisted for a shorter length of time on both DT tasks (PASAT-C: $F=3.9, p$ $<.05$; MTPT-C: $F=5.2, p<.05)$. Further, low DT (PASAT-C: Wald $=4.07, p<.05$; OR $=0.67$; MTPT-C : Wald $=5.01, p<.05 ; \mathrm{OR}=0.64)$ predicted ASPD diagnosis after accounting for BPD, MDD, and past year polysubstance use. This study suggests that male substance users with ASPD demonstrate significantly less behaviorally-assessed DT than male substance users without ASPD.

This study was strengthened by accounting for BPD, MDD, and past year polysubstance use. Additionally, this study had good representation of individuals with 
ASPD (36\%). However, this study used an entirely male sample. So, further study is needed to know if similar patterns are evident in females.

Antisocial and psychopathic traits. Building on the study above, Sargeant, Daughters, Curtin, Schuster, and Lejuez (2011) examined the role of ASPD diagnosis (as determined by diagnostic interview) and psychopathic traits (as determined by the Psychopathic Personality Inventory, as cited in Sargeant et al., 2011, p. 2) in DT in a sample of substance-dependent inpatients $(N=107,85 \%$ male, $88 \%$ African American, mean age $=41$ ). For, the authors held that individuals diagnosed with ASPD represent a group of individuals who are heterogeneous in level of psychopathic traits (i.e., some individuals with ASPD have higher levels of psychopathic traits than other individuals with ASPD). The Psychopathic Personality Inventory includes subscales measuring the following traits: "impulsive nonconformity, blame externalization, Machiavellian egocentricity, carefree nonplanfulness, stress immunity, social potency, fearlessness, and coldheartedness" as well as two subscales (Unlikely Virtues and Deviant Responding) which measure impression management (Sargeant et al., 2011, p. 2). Distress tolerance was assessed behaviorally through the PASAT-C and MTPT-C. Results indicated that individuals with an ASPD diagnosis persisted for less time $\left(F(1,103)=4.16, p=.044, \eta^{2}\right.$ $=.04, \beta=-.21)$ and were less likely to complete the DT tasks (Wald $(1)=6.78, p=.009)$ than individuals without an ASPD diagnosis. Further, higher levels of psychopathic traits were associated with higher DT persistence $\left(F(1,103)=3.97, p=.049, \eta^{2}=.04, \beta=\right.$ .21 ) and higher likelihood of DT task completion (Wald $(1)=5.31, p=.021)$. These calculations controlled for gender, age, education level, and race. Specifically, deviant 
responding $(\beta=.32, p=.009)$ and cold-heartedness $(\beta=.34, p=.015)$ were associated with DT persistence when controlling for ASPD traits.

So, this study and the study by Daughters et al. (2008) both demonstrated that among inpatients with SUDs, having an ASPD diagnosis is associated with lower DT. This relationship appears to be more nuanced, however, if an individual has both an ASPD diagnosis and certain psychopathic traits. For, in this study, the psychopathic traits of cold-heartedness and deviant responding were associated with higher DT. This finding may suggest that individuals with ASPD who are also low in psychopathic traits may exhibit lower DT than individuals with ASPD who have higher levels of psychopathic traits. In other words, when it comes to DT level and the impulsive actions which are associated with low DT, certain psychopathic traits may have protective value for individuals with ASPD.

The influence of parenting

Rutherford, Booth, Luyten, Bridgett, and Mayes (2015) studied the relationship between mothers' DT and level of parental reflective functioning (the parent's awareness of their child's emotions and how the emotions impact behavior). Participants ( $N=62$, 53\% African American, mean age = 27) completed one self-report (sDTS) and two behavioral tasks (PASAT-C and Baby Stimulator Paradigm) to assess DT. Results indicated that lower self-reported DT (sDTS: Tolerance: $r=-.38, p<.01$; sDTS: Absorption: $r=-.49, p<.01$; sDTS: Appraisal: $r=-.42, p<.01$; sDTS: Regulation: $r=$ $.20, n s$ ) was correlated with higher levels of pre-mentalizing about infant emotions (when the mother attributes the child's behavior to her own emotions, rather than engaging in reflective functioning about the child's emotions). Mothers who persisted through the 
whole baby stimulator task reported lower levels of pre-mentalizing than mothers who did not complete the task $(t(20)=2.04, p=.05)$. However, persistence on the PASAT-C was not correlated with level of pre-mentalizing $(r=.03, n s)$. This study thus suggests that mothers who pre-mentalize about their child's emotions may be less tolerant of their child's distress and likewise less tolerant of their own distress in response to their upset child. Though this study did not assess the impact of parental distress on the infant (since it was a simulated doll), the low levels of reflective functioning may be an avenue for the impact of parental DT on child DT.

Daughters, Gorka, Rutherford, and Mayes (2014) examined the impact of maternal DT on adolescent DT. Participants $(N=139,53 \%$ male, mean age $=16)$ and their biological mothers (mean age $=46$ ) completed the PASAT-C to behaviorally assess DT. Analysis controlled for the mothers' age and level of depression. Results indicated a significant interaction between maternal DT and gender of the adolescent in the prediction of adolescent DT $(B=-2.38, \mathrm{p}<.05)$, such that mothers with low DT had higher probability of having an adolescent daughter with low DT $(B=1.77, \mathrm{p}<.05)$. However, maternal DT was not related to adolescent son DT $(B=-0.60, n s)$. As such, the level of maternal DT appears to predict the level of DT in adolescent daughters, but not in adolescent sons.

The influence of biological factors

Amstadter et al. (2012) examined genetic associations with performance on a behavioral measure of DT (Behavioral Indicator of Resilience to Distress) among adolescents $(N=277,55 \%$ male, $51 \%$ Caucasian, mean age $=12)$. Results indicated that individuals carrying the Val allele of COMT Val ${ }^{158}$ Met polymorphism (rs4680), which is 
linked to lower cognitive efficiency and increased immediate reward bias, were more likely to quit the task than those without Val allele. Individuals with copies of the s allele of the 5-HTTLPR polymorphism, which is linked to lower emotional resilience and greater emotional disinhibition, were also more likely to quit the task than those without. For each risk allele the individual had, his/her chance of quitting the task increased 1.75 fold $(\mathrm{OR}=1.75,95 \mathrm{CI}[1.22-2.51], p=.003)$. This effect was moderated by the presence of a history of emotional abuse, such that individuals with the risk alleles and a history of emotional abuse were most likely to quit the task $(\mathrm{OR}=1.54, p=.04)$. Though an exploratory study, this research suggests that DT level may be influenced by certain genetic variants, though the influence is moderated by a situational factor (a history of emotional abuse).

Chiappelli et al. (2014) examined the role of an endogenous neuromodulator, kynurenic acid, in DT among individuals with and without schizophrenia. They also assessed processing speed and working memory, to assess the role of cognition in DT and schizophrenia. Participants $(N=128)$ completed two behavioral tasks to measure DT (PASAT-C and MTPT-C). The sample was composed of outpatient individuals with schizophrenia $(N=64,66 \%$ male, mean age $=38)$ and nonclinical, healthy controls $(N=$ $64,55 \%$ male, mean age $=39$ ). Results indicated that individuals with schizophrenia were more likely to be distress intolerant (defined as quitting both behavioral tasks) $\left(\chi^{2}=\right.$ $8.55, p=.003)$. Among individuals with schizophrenia, those who were distress intolerant had lower processing speed $\left(t_{62.9}=4.29, p<.001\right)$, but not lower working memory $(p=.10)$, than those who were not distress intolerant. However, this difference was not present in the control group. Further, distress intolerant individuals with 
schizophrenia had greater kynurenic acid in their saliva following the behavioral tasks than individuals with schizophrenia who had high DT $\left(\mathrm{F}_{1,54}=6.25, p=.02\right)$ and also controls $\left(\mathrm{F}_{1,74}=6.02, p=.02\right)$. Again, this difference was not present in the control group.

Given that the relationship between kynurenic acid and distress intolerance was found only among individuals with schizophrenia, the authors suggested that the relationship is specific to the distress intolerant individuals with schizophrenia. However, the representation of distress intolerant individuals was small (12 healthy controls, 22 individuals with schizophrenia), especially in the control group. So, these findings would be strengthened by replication in a larger sample.

\section{Emotional DT Summary}

In summary, self-reported emotional DT is represented by a single higher-order factor with four lower-order factors: absorption, appraisal, regulation, and tolerance. Distress tolerance is quite stable across time, unless deliberate attempts to change DT level are made. Initial evidence suggests that targeted interventions drawn from CBT and DBT as well as DT-specific interventions may increase DT level. Additionally, DT is related to, but separate from avoidance and urgency. Further, although DT is impacted by a number of factors (e.g., emotional regulation, attentional control, mindfulness, personality traits, parenting, and biology), unexplained individual differences in DT remain. 


\section{Emotional Reactivity Overview}

\section{History and Definition of Emotional Reactivity}

As with DT, definitions of emotional reactivity differ in scope. For instance, Nock et al. (2008) defined emotional reactivity broadly as

The extent to which an individual experiences emotions (a) in response to a wide array of stimuli (i.e., emotion sensitivity), (b) strongly or intensely (i.e., emotion intensity), and (c) for a prolonged period of time before returning to baseline level of arousal (i.e., emotion persistence). (p. 107)

This broad definition is represented in the authors' measure, the Emotion Reactivity Scale (ERS). Whereas, Cougle, Timpano, Sarawgi, Smith, and Fitch (2013) defined emotional reactivity more narrowly as the "intensity of response to an emotionally salient stimulus" (p. 478). This narrower definition is represented in the Affect Intensity Scale (Bryant, Yarnold, \& Grimm, 1996, as cited in Bornovalova et al., 2011, p. 747). It is important to note the difference in scope of the definition when considering the research conducted with these scales reviewed below.

In addition to differences in scope, emotional reactivity must also be distinguished from negative affectivity. Negative affectivity refers to the degree of negative emotions an individual experiences. Whereas, emotional reactivity refers to the degree to which an individual experiences both negative and positive emotions. This distinction is particularly important in consideration of individuals with BPD. For, research suggests that it is neutral stimuli, not negative stimuli, to which individuals with BPD react more strongly than controls (Herpertz et al., 1999, as cited in Hawkins, Macatee, Guthrie, \& Cougle, 2013, p. 444).

In this section, I will first review the factor structure of emotional reactivity and the relationship between emotional reactivity and self-damaging behaviors. Then, I will 
review evidence for and against emotional reactivity as an individual difference factor in DT.

\section{Emotional Reactivity Factor Structure}

In developing the ERS, Nock et al. (2008) examined the underlying factor structure of emotional reactivity. The authors anticipated, based on their definition listed above, that factor analysis would reveal three components of emotional reactivity: emotion sensitivity, emotion intensity, and emotion persistence. In their sample of outpatients ranging from 12 to 19 years old $(N=94,78 \%$ female, $72 \%$ Caucasian, mean age $=17)$, EFA revealed three factors which together accounted for $57.8 \%$ of the variance in scores. However, that the first factor accounted for the majority of the variance (43.4\%), that all of the items loaded $(\geq .44)$ on the first factor, the high correlation between the three factors, and the lack of distinguishable content themes between the factors suggested that emotional reactivity may be best represented by a single factor. Further, the total score $(\alpha=.94)$ had strong internal consistency. In this study, scores on the ERS were not significantly associated with age $(r=.20, n s)$ or gender $\left(t_{85}=1.87, n s, d\right.$ $=0.28)$. The ERS also demonstrated strong internal consistency $(\alpha=.92)$ in a study conducted by Franklin, Puzia, Lee, and Prinstein (2014) with a nonclinical sample of individuals with a history of NSSI $(N=49,73 \%$ female, $61 \%$ Caucasian, mean age $=24)$.

Claes, Smits, and Bijttebier (2014) conducted CFA on a Dutch version of the ERS among a sample of high school students $(N=651,61 \%$ female, mean age $=16)$. Both the 1 -factor model $(\mathrm{AIC}=2440.93, \mathrm{SRMR}=.08, \mathrm{CFI}=.94)$ and 3 -factor model $(\mathrm{AIC}=$ 2429.79, $\mathrm{SRMR}=.09, \mathrm{CFI}=.93)$ achieved good fit in this population. However, since the 3-factor model did not achieve a better fit than the 1-factor model, the 1-factor model 
was retained $\left(\chi^{2}(12)=13.14, p=.36\right)$. This conclusion was also supported by high correlation between the three factors. The total scale again demonstrated strong internal consistency in this sample $(\alpha=.95)$.

Lannoy et al. (2014) conducted CFA on a French adaptation of the ERS among a nonclinical sample of adults $(N=258,78 \%$ female, mean age $=38)$. They tested a 1 factor model, a 3-factor model, and a hierarchical model (with a single-factor solution as a second-order latent variable). All three models demonstrated good fit for the data (1factor model: $\mathrm{GFI}=.95, \mathrm{AIC}=1136.60, \mathrm{BCC}=1345.03, \mathrm{ECVI}=5.20 ; 3$-factor model: $\mathrm{GFI}=.95, \mathrm{AIC}=1346.20, \mathrm{BCC}=1354.06, \mathrm{ECVI}=5.24 ;$ Hierarchical model: $\mathrm{GFI}=.95$, $\mathrm{AIC}=1336.48, \mathrm{BCC}=1344.54, \mathrm{ECVI}=5.20)$. The authors decided to retain the hierarchical model, based on the Brown-Cudeck Criterion value. However, comparative statistics were not run to indicate whether it was a better fit than the 1-factor model (which had very similar fit statistics). The total score again achieved strong internal validity $(\alpha=.94)$. In this study, scores on the ERS total scale and subscales were not significantly associated with age $(r=-.003$ to $-.999, \mathrm{p}=.114$ to .961$)$.

\section{Relationship Between Emotional Reactivity and Psychological Disorders}

Nock et al. (2008) examined the relationship between emotional reactivity and the presence and number of psychological disorders in a clinical sample. The presence and number of psychological disorders was determined by diagnostic interview based on the Diagnostic and Statistical Manual of Mental Disorders, fourth edition (American Psychiatric Association, 2013). Results indicated elevated emotional reactivity among participants with a mood disorder compared to participants without a mood disorder $(t=-$ 
$5.53, p<.001, d=1.20)$, participants with an anxiety disorder compared to participants without an anxiety disorder $(t=4.88, p<.001, d=1.06)$, and participants with an ED compared to individuals without an $\mathrm{ED}(t=-4.30, p<.001, d=0.94)$. Emotional reactivity was also higher among participants with NSSI within the past year $(t=-4.66, p$ $<.001, d=1.01)$, among participants with suicidal ideation within the past year $(t=-4.03$ $p<.00, d=0.88)$, and among participants with a suicide attempts within the past year $(t$ $=-2.95, p<.01, d=0.64)$ compared to individuals without these behaviors. However, emotional reactivity was not elevated among participants with an $\operatorname{SUD}(t=-1.48, n s, d=$ 0.32 ) or disruptive behavior disorder ( $t=0.08, n s, d=0.02$ ). The authors suggested that this difference may indicate that emotional reactivity is specifically associated with certain psychological diagnoses (e.g. mood disorders, EDs, and NSSI), rather than broad psychopathology. However, they also noted that the heterogeneity of SUDs and disruptive behavior disorders may have masked relations between specific subgroups of these disorders and emotional reactivity.

Further, emotional reactivity fully mediated the relationship between the number of psychological disorders (mood, anxiety, and EDs only, which were coded as a sum from 0 to 3 ) and the presence of NSSI within the past year (Sobel $z=2.58, p<.01$ ) and also the presence of suicidal ideation within the past year (Sobel $z=2.16, p<.05$ ). Having a higher number of psychological disorders was associated with higher emotional reactivity $(\beta=.63, p<.001)$, which was associated with the presence of NSSI within the past year $(\beta=.37, p<.01)$. Likewise, having a higher number of psychological disorders was associated with higher emotional reactivity $(\beta=.63, p<.001)$, which was associated with the present of suicidal ideation within the past year $(\beta=.40, p<.001)$. However, 
emotional reactivity did not mediate the relationship between the number of psychological disorders and the presence of suicide attempts within the past year (Sobel $z$ $=0.99, n s)$. The authors suggested that the lack of mediation may be due to the lower representation of suicide attempts or may be due to there being a greater number of factors influencing suicide attempts than there are factors influencing NSSI and suicidal ideation. However, further research is needed to clarify the difference.

\section{Evidence Supporting Emotional Reactivity as} an Individual Difference Factor in DT

As indicated above, Lynch and Mizon (2011) hypothesized that high emotional reactivity is a contributing factor to low DT. The study of emotional reactivity as an individual difference influencing DT was also suggested by Leyro, Zvolensky, and Bernstein (2010), who stated,

Although there are numerous factors that could influence distress tolerance, one useful starting point, in terms of theory, would be to direct scientific attention to individual differences in the tendency to experience positive and negative mood states (Watson, 2000). Individual differences in emotionality are directly relevant to the study of distress tolerance in that they may influence the development and maintenance of distress tolerance (e.g., more frequent or intense emotional experience would require more frequent or greater degree of use of distress tolerance skills and resources). (p. 594)

In this section, I will review research supporting emotional reactivity as a potential individual difference factor in DT in the context of BPD symptoms, depression symptoms, heavy drinking, NSSI, obsessive-compulsive symptoms, panic symptoms, emotional response to research protocols, and worry symptoms. 


\section{DT and Emotional Reactivity in Borderline Symptoms}

Bornovalova et al. (2011) examined the role of negative emotionality, negative affect intensity, and DT in BPD traits. The sample was drawn in part from community members who were smokers $(N=110,53 \%$ male, $70 \%$ African American, mean age $=$ 47) and in part from inpatients receiving treatment for Substance Used Disorders $(N=76$, $67 \%$ male, $80 \%$ African American, mean age $=42$ ). Affect intensity was measured through self-report on the Affect Intensity Measure, intensity subscale (Bryant, Yarnold, \& Grimm, 1996, as cited on p. 747). Distress tolerance was assessed behaviorally through the PASAT-C and MTPT-C.

When considered individually, negative emotionality $(\beta=.52, p<.001)$ was predictive of borderline trait severity after controlling for the number of SUDs and level of education. However, DT was not predictive of borderline trait severity after controlling for the same variables $(\beta=-.04, n s)$. When considered in combination, negative emotionality interacted with DT in the prediction of borderline traits $(\beta=-.25, p<.01)$ : negative emotionality predicted borderline traits at all levels of DT, but, the highest level of borderline traits occurred at high levels of negative emotionality and low levels of DT.

Further, when controlling for negative emotionality, affect intensity $(\beta=-.02, n s)$ and DT $(\beta=-.06, n s)$ did not predict borderline trait levels when considered individually. However, when considered together, DT and affect intensity interacted in the prediction of borderline traits $(\beta=-.21, p<.05)$ : the highest level of borderline traits occurred at high levels of affect intensity and low levels of DT.

Thus, affect intensity and DT interacted in the prediction of borderline traits, even when controlling for the impact of negative emotionality. Of note, the authors reran the 
data including the affect reactivity scale of the Affect Intensity Scale and found the same pattern of results. So, it appears that affect reactivity similarly interacted with DT in the prediction of borderline traits. This result supports the idea of a unitary factor structure for emotional reactivity, since affect intensity and affect reactivity interacted with DT in the same way.

\section{DT and Emotional Reactivity in Depression Symptoms}

Ellis et al. (2010) examined emotional reactivity and DT among undergraduate students who were divided into two groups based on level of self-reported dysphoria. The dysphoric group $(N=28,73 \%$ female, $71 \%$ Caucasian, mean age $=22)$ scored above 20 on the Beck Depression inventory, second edition (Beck, Steer, \& Brown, 1996, as cited on p. 598), whereas the non-dysphoric group $(N=35,53 \%$ female, $74 \%$ Caucasian, mean age $=23)$ scored 12 or below. Distress tolerance was assessed behaviorally through the PASAT-C and the MTPT-C, where DT was defined as latency to termination. Emotional reactivity was assessed through self-report on the Profile of Mood States-Short Form (Curran, Andrykowski, \& Studts, 1995, as cited on p. 597).

Results indicated that individuals in the dysphoric group $\left(F=41.26, p=.00, \eta^{2}=\right.$ .62) reported greater increases in anger following the PASAT-C than the non-dysphoric $\operatorname{group}\left(F=16.53, p=.00, \eta^{2}=.33\right)$. The dysphoric group $\left(F=19.72, p=.00, \eta^{2}=.44\right)$ also reported greater increases in ager following the MTPT-C than the non-dysphoric group $\left(F=26.87, p=.00, \eta^{2}=.45\right)$. The dysphoric group did not differ from the nondysphoric group in increases in anxiety $\left(F=0.54, n s, \eta^{2}=.01 ; F=0.28, n s, \eta^{2}=.01\right)$ and sadness $\left(F=0.23, n s, \eta^{2}=.00 ; F=1.17, n s, \eta^{2}=.02\right)$ following the PASAT-C and the 
MTPT-C (respectively). The groups did not differ in latency to termination on the PASAT-C $\left(F=0.00, n s, \eta^{2}=.00\right)$. However, the dysphoric group demonstrated less DT on the MTPT-C than the non-dysphoric group $\left(F=4.14, p=.05, \eta^{2}=.07\right)$.

This study found that the group for which a psychological task produced more anger demonstrated less task persistence. However, emotional reactivity and DT were both assessed as outcome variables so the relationship between them was not examined. Additionally, this study produced a difference in DT on only one out of two DT challenges, and that observed difference was only marginally significant. So, the results would need to be upheld with a more robust difference in DT observed.

Ellis, Vanderlind, and Beevers (2013) conducted a similar study with a younger sample of undergraduate students who were classified as diagnosed with $\operatorname{MDD}(N=74$, $66 \%$ female, $49 \%$ Caucasian, mean age $=23)$ or not diagnosed with MDD $(N=107,65 \%$ female, $45 \%$ Caucasian, mean age $=19$ ). Distress tolerance was assessed behaviorally through the MTPT-C, where DT was defined as latency to termination. Emotional reactivity was again assessed through self-report on the Profile of Mood States-Short Form. Results again indicated that the MDD group $\left(F=48.44, p=.00\right.$, partial $\left.\eta^{2}=.41\right)$ experienced greater increases in anger during the task than the non-MDD group $(F=$ 41.93, $p=.00$, partial $\eta^{2}=.29$ ) and demonstrated less task persistence (DT) than the nonMDD group $\left(F=7.58, p=.01, \eta^{2}=.04\right)$. In this study, the interaction of depression status and anger reactivity was significant $(B=-0.41, \beta=-0.44, t=-3.51, p=.0001)$ : for individuals with MDD, anger reactivity was negatively associated with DT. However, for individuals without MDD, anger reactivity was positively associated with DT. 


\section{DT and Emotional Reactivity in Heavy Drinking}

Winward et al. (2014) examined emotional reactivity and DT among heavy episodic drinking youth (HEDs; $N=23)$ and controls $(N=23)$ after varying abstinence intervals ( $50 \%$ female, $74 \%$ Caucasian, mean age $=18)$. Distress tolerance was assessed behaviorally on the MTPT-C. Distress tolerance was defined as latency to termination and emotional reactivity was measured as the difference between pre-test and post-test affect. This task was completed at three time points: the first time point was within 10 days $(M=4.26, \mathrm{SD}=4.43)$ after a heavy drinking episode. The second time point occurred two weeks $(M=18.77, \mathrm{SD}=4.96)$ after the first time point, and the third time point occurred four weeks $(M=32.12, \mathrm{SD}=4.55)$ after the first time point

Results indicated that at time point 1 , HEDs experienced an $81 \%$ greater increase in frustration $(b=24.52, z=3.28, p=.001)$ than controls, an $86 \%$ greater increase in irritability $(b=13.95, z=2.22, p=.026)$, and a $320 \%$ greater reduction in happiness $(b=$ $-24.65, z=-4.14, p=.001)$ than controls. The groups did not differ in their experience of anxiety. Heavy episodic drinking youth experienced less increase in frustration and irritability in response to the task at time point 2 (only a trend, frustration: $b=-14.22, z=$ $-1.84, p=.066$, irritability: $b=-12.00, z=-1.88, p=.061$ ) and time point 3 (frustration: $b$ $=-19.95, z=-2.56, p=.011$, irritability: $b=-20.64, z=-3.20, p=.001)$ than at time point

1. They also experienced less reduction in happiness in response to the task at time point 2 (only a trend, $b=-10.36, z=1.54, p=.125)$ and time point three $(b=16.78, z=2.46, p$ $=.014)$ than at time point 1 . For controls, their level of frustration, irritability, and happiness did not differ over time points. Heavy episodic drinking youth demonstrated less DT than controls at time point $1(b=-108.28, z=-2.11, p=.035$, with the control 
group persisting $53 \%$ longer than the HEDs. However, the two groups did not differ in DT at time points 2 and 3.

This study suggested that "adolescents with recent heavy episodic drinking (i) display greater negative affect responses and poorer distress tolerance in cognitively challenging situations during early abstinence and (ii) become less emotionally reactive as abstinence continues" (Ellis et al., 2013, p. 1766). However, the study did not assess expectations of the participants, so the decrease in emotional reactivity may have instead been due to changing expectations based on prior exposure to the task. In this study, it appears that emotional reactivity and DT follow a similar pattern in the abstinence process for HEDs. However, the relationship between emotional reactivity and DT was not directly assessed. Further, the sample size was relatively small and so the study should be improved with a larger sample.

\section{DT and Emotional Reactivity in NSSI}

Nock and Mendes (2008) examined physiological reactivity and DT in NSSI among adolescents with a history of NSSI $(N=62,78 \%$ female, $75 \%$ Caucasian, mean age $=17)$ and controls $(N=30,73 \%$ female, $70 \%$ Caucasian, mean age $=17)$. Physiological reactivity was measured via changes in skin conductance. Distress tolerance was assessed behaviorally through the Distress Tolerance Test (DTT; developed for this study), where DT was defined as task persistence. Results indicated that the NSSI group exhibited greater changes in skin conductance over the course of the DT task than controls $(F(1,81)=6.61, p<.05, d=0.57)$. Of note, the difference in reactivity between groups did not emerge until the $8^{\text {th }}$ minute of the task. This difference remained true after controlling for MDD, post-traumatic stress disorder, and attention- 
deficit/hyperactivity disorder $(F(1,78)=4.43, p<.05)$. Further, individuals in the NSSI group demonstrated lower DT than controls $(t(90)=2.47, \mathrm{p}<.05, d=0.52)$. This study thus suggests that physiological reactivity and DT both differentiated between individuals with a history of NSSI and individuals without a history of NSSI. This supports the idea that physiological reactivity and behaviorally-assessed DT may be related to one another. However, the relationship between emotional reactivity and DT was not directly compared.

\section{DT and Emotional Reactivity in Obsessive-Compulsive Symptoms}

Cougle et al. (2013) investigated the role of emotional reactivity and DT in obsessive-compulsive symptoms among undergraduate students $(N=167,64 \%$ female, $76 \%$ Caucasian, mean age $=19)$. Participants were shown four video clips intended to elicit fear, sadness, disgust, and anger. Emotional intensity was measured as the sum of self-reported peaks for each emotion across the films. Emotional DT was assessed through self-report by four author-created, Likert-scale questions such as "Rate the degree to which you were able to focus on the clip without turning away or distracting yourself” (p. 482). Emotional DT was also assessed behaviorally, with the MTPT-C. Physical distress tolerance was assessed behaviorally with the Handgrip Dynamometer Endurance Task. Distress tolerance was measured as latency to termination on both behavioral tasks. For comparison purpose, participants were divided into high and low obsession symptom groups.

Results indicated that, in comparison to the low obsession group, the high obsession group reported greater emotional intensity (both $p<.01$ ) on the sad and fear 
films as well as lower self-reported DT (all $p<.001$ ) in response to the sad, angry, and fear films. The high obsession group also self-reported lower overall emotional DT than the low obsession group, when controlling for gender, baseline anxiety, and baseline depression $\left(p<.05, \eta^{2}=.08\right)$. Additionally, lower behaviorally-assessed emotional DT was associated with greater obsession symptoms $(r=-.24, p<.01)$, but was not associated with compulsions (e.g., checking $(r=-.00, n s)$, neutralizing $(r=.02, n s)$, ordering $(r=-.12, n s)$, or washing $(r=-.09, n s)$. This association between behaviorallyassessed emotional DT and obsession symptoms held true after controlling for gender, baseline anxiety, baseline depression, and errors per second on the DT task. Lower behaviorally-assessed physical distress tolerance was also associated with greater obsession symptoms while controlling for depression, but only among males $(\beta=-.42, t=$ $-2.49, p<.02)$.

Thus, in this study, higher emotional intensity (sad and fear films only), lower self-reported emotional DT, lower behaviorally-assessed emotional DT, and lower behaviorally-assessed physical distress tolerance (men only) were associated with greater obsessive symptoms. Thus, both high emotional reactivity and low DT were associated with obsessive (but not compulsive) symptoms. However, the interaction between emotional reactivity and DT was not assessed. The study was strengthened by the assessment of multiple forms of DT in response to an in-vivo task. However, the study was weakened by the use of a novel assessment of emotional DT without a comparison assessment. A further limitation was that the film intended to elicit fear elicited greater anger than fear. 


\section{DT and Emotional Reactivity in Panic Symptoms}

Marshall et al. (2008) examined panic reactivity, physiological responsivity, and physical distress tolerance among daily smokers $(N=95,58 \%$ female, $92 \%$ Caucasian, mean age $=29$ ). Participants engaged in two voluntary hyperventilation challenges. After the first, panic reactivity was measured by the Diagnostic Sensations Questionnaire (Sanderson, Rapee, \& Barlow, 1988, 1989, as cited on p. 6) which assesses for selfreported panic attack symptoms. During the second hyperventilation challenge, physical distress tolerance was behaviorally assessed as latency to termination on the second hyperventilation challenge. Based on the results of the Diagnostic Sensations Questionnaire, the sample was divided into panic attack status groups (yes or no). To assess physiological responsivity, heart rate and respiration rate data were collected in the last minute prior to the initial hyperventilation challenge, and in the last minute of the initial hyperventilation challenge. Positive panic attack status was correlated with low DT $(r=-.25, p<.05)$, high negative affectivity $(r=.31, p<.01)$, and high AS $(r=.30, p<$ $.01)$. Additionally, panic attack status $(t=-2.44, p<.05, \beta=-.26)$ was predictive of DT after accounting for AS, negative affectivity, DI, and number of cigarettes smoked per day $\left(F=2.92, p<.05, R^{2}=.14\right)$. Panic attack status accounted for $5.8 \%$ of the variance in DT, beyond the rest of the model $(p<.05)$. Thus, individuals who endorsed having a panic attack after the first hyperventilation challenge demonstrated lower DT than individuals who did not endorse a panic attack.

Interestingly, individuals who endorsed having a panic attack did not differ from individuals who did not endorse having a panic attack on levels of physiological responsivity (first challenge: heart rate: $F(1,90)=1.44, n s$; respiration rate: $F(1,75)=$ 
$0.25, n s)$ (second challenge: heart rate: $F(1,86)=2.07, n s ;$ respiration rate: $F(1,74)=$ $0.09, n s)$. So, it appears that individuals who endorsed having a panic attack differed in perceptions of physiological sensations rather than in objective measurement of the sensations.

Thus, in this study, self-reported panic reactivity, a marker of emotional reactivity, was predictive of behaviorally-assessed physical distress tolerance level. Though this study only included physical distress tolerance without a measure of emotional DT, it was included in this literature review since it addressed the interaction of DT and emotional reactivity in panic symptoms. After thorough review of the extant literature, it does not appear that this relationship has yet been studied with a measure of emotional DT.

\section{DT and Emotional Reactivity in Research Response}

Shorey et al. (2013) examined the relationship between DT and emotional reactivity in response to participating in research regarding the topic of dating violence. The sample was composed of undergraduate females $(N=282,84 \%$ Caucasian, mean age =18). The participants first completed an assessment of dating violence experiences (victim of physical violence, perpetrator of physical violence, victim of psychological violence, and perpetrator of psychological violence) in the past 12 months and a selfreport of emotional DT (the sDTS). Then, they completed a measure of negative emotional reactions to the research participation via the Reactions to Research Participation Questionnaire (Newman et al., 2001, as cited on p. 482). 
Results indicated that lower DT was predictive of more negative emotional reactions to research participation, when considering status as a psychological victim or perpetrator $(B=.14, p=.02)$ and as a physical victim or perpetrator $(B=.15, p=.01)$. Distress tolerance was not associated with perception of research benefits $(r=-.03, n s)$, research drawbacks $(r=.00, n s)$, research cost-benefit ratio $(r=.04, n s)$, or global evaluation $(r=.03, n s)$. This study thus suggests a specific interaction between DT and emotional reactivity in response to research participation.

\section{DT and Emotional Reactivity in Worry}

Macatee et al. (2015) examined the role of DT and emotional reactivity in relation to worry symptoms among undergraduate students $(N=87,76 \%$ female, $67 \%$ Caucasian, mean age $=19)$. Participants completed measures at two time points that were 1 month apart. Baseline self-reported emotional DT was assessed through the sDTS. Baseline negative affect was assessed through the negative affect subscale of the Positive and Negative Affect Schedule (PANAS; Watson, Clark, and Tellegen, 1988, as cited on p. 454). In between time point 1 and time point 2, participants completed daily diaries two times a week. Daily DT was assessed through the Distress Tolerance Scale-Daily (Hawkins et al., 2013, as cited on p. 455). Daily negative affect (operationalized as emotional reactivity) was assessed through the negative affect subscale of the PANAS short form (Mackinnon et al., 1999, as cited on p. 455).

Results indicated that baseline DT and baseline negative affect were negatively correlated $(r=-.42, p<.001)$, as were Time 2 DT and Time 2 negative affect $(r=-.25, p$ $<.05)$. Unfortunately, correlations between DT and daily negative affect were not reported. Further, lower baseline DT predicted both higher daily worry $(t=-2.15, p=$ 
$.034)$ and higher Time 2 worry $(\beta=.07, p=.50)$. Daily negative affect (emotional reactivity) $(t=8.78, p<.001)$, but not baseline negative affect $(t=1.18, n s)$, predicted daily worry. Unfortunately, neither the relationship between daily negative affect and daily DT, nor the relationship between daily negative affect and Time 2 DT were reported. Thus, this study suggests that both lower baseline DT and higher daily negative affect are associated with higher daily worry. However, the direct relationships between DT and emotional reactivity were not reported. Additionally, daily negative affect as measured by the short form PANAS may reflect only the presence of negative affect, to the exclusion of the intensity component of emotional reactivity.

\section{Evidence Opposing Emotional Reactivity as an Individual Difference Factor in DT}

Other researchers have argued against the role of emotional reactivity in distress tolerance. For instance, Macatee and Cougle (2015) stated that low DT "is primarily reflective of negative affective responses to distress and strong action-tendencies to seek immediate negative reinforcement rather than negative emotional reactivity per se" (p. 37). In this section, I will review research opposing emotional reactivity as a potential individual difference factor in DT in the context of anxiety symptoms, NSSI, and smoking.

\section{DT and Emotional Reactivity in Anxiety}

Macatee and Cougle (2013) examined the role of DT and emotional reactivity in generalized, health, and social anxiety among undergraduate students $(N=122,59 \%$ female, $78 \%$ Caucasian, mean age $=19$ ). The protocol utilized was the same as that reported for Cougle et al. (2013) above, except that physical distress tolerance was not 
measured. (In this study, DT was scored such that a high score indicated low distress tolerance. This is reflected in the correlation coefficients listed below).

In response to the anger film, lower emotional DT was associated with greater social anxiety $(r=.24, \mathrm{p}<.01)$, and greater anger intensity was associated with greater generalized anxiety $(r=.26, \mathrm{p}<.01)$. In response to the fear film, emotional DT and emotional intensity were not associated with anxiety symptoms. In response to the disgust film, lower emotional DT was associated with greater generalized $(r=.28, \mathrm{p}$ $<.01)$ and social anxiety $(r=-.24, \mathrm{p}<.01)$. But, greater disgust intensity was not associated with anxiety symptoms. In response to the fear film, lower emotional DT and fear intensity were not associated with anxiety symptoms. In response to the films overall, lower emotional DT was associated with higher social anxiety $(r=.20, \mathrm{p}<.01)$ and higher emotional reactivity was associated with higher generalized anxiety $(r=.32, \mathrm{p}$ $<.01)$.

Social anxiety $(\beta=.23, p<.05)$, but not health anxiety $(r=.20, n s)$ or generalized anxiety $(\beta=.12, n s)$, predicted variance in emotional DT, after controlling for gender, baseline anxiety, and baseline depression. Generalized anxiety $(\beta=.28, p<.01)$, but not health anxiety $(r=.19, n s)$ or social anxiety $(\beta=.08, n s)$, predicted variance in emotional reactivity, after controlling for the same variables. Behaviorally-assessed DT was not associated with emotional reactivity or emotional DT ( $p$ 's $>.6)$, or any anxiety measure $(r$ 's $<.22, p$ 's $>.02)$. In this study, emotional reactivity was related to generalized anxiety symptoms, but emotional DT related to social anxiety symptoms. Though both emotional reactivity and emotional DT interacted with anxiety symptoms, their patterns of associations differed. This suggests differing contributions of emotional reactivity and 
emotional DT to anxiety. However, as emotional reactivity and emotional DT were not directly compared, further research is needed.

\section{DT and Emotional Reactivity in Smoking}

Zvolensky, Feldner, Eifert, and Brown (2001) examined AS, emotional reactivity, and DT among undergraduate students who smoked at least 20 cigarettes a day $(N=22$, $55 \%$ female, $91 \%$ Caucasian, mean age $=21$ ). The sample was categorized as individuals whose last quit attempt was sustained more than 7 days and individuals whose last quit attempt was sustained less than 7 days. This study assessed physical DT only, but was included in the literature review because no studies could be found that included emotional reactivity and smoking cessation in the context of emotional DT. Physical distress tolerance was assessed behaviorally through a carbon-dioxide enriched air challenge, in which physical distress tolerance was measured by persistence at the task. Emotional reactivity was measured by a pictorial self-report completed 30 seconds postinhalation (Self-Assessment Manikin; Lang, 1980, as cited on p. 907).

Results indicated that the groups did not differ in level of tolerance of physical distress ( $\geq 7: M=32.2, \mathrm{SD}=8.9 ;<7: M=28.5, \mathrm{SD}=11.6)$ nor level of AS $(\geq 7: M=$ 20.1, $\mathrm{SD}=11.1 ;<7: M=23.2, \mathrm{SD}=9.8)$. However, individuals whose last quit attempt was sustained less than 7 days $(M=5.7, \mathrm{SD}=1.7)$ reported greater emotional reactivity than those whose last quit attempt was sustained more than 7 days $(M=4.4, \mathrm{SD}=0.9, p$ $<.01, \eta^{2}=0.30$ ). So, in this study, emotional reactivity, but not physical distress

tolerance, differed between group membership. However, this study utilized tolerance of physical discomfort rather than tolerance of psychological discomfort. Additionally, the sample size was small so results must be replicated with a larger sample. 


\section{Emotional Reactivity Summary}

In summary, emotional reactivity appears to be a single factor that is related to several serious psychological disorders (e.g., mood, anxiety, and eating disorders) and to serious behavioral symptoms (e.g., NSSI and suicidal ideation). Additionally, emotional reactivity appears likely to be a contributing individual difference factor in DT and appears to relate to a wide variety of negative behaviors (e.g., borderline personality symptoms, depression symptoms, heavy drinking, NSSI, and various anxiety symptoms) in a similar way as DT. There are very few studies that suggest that emotional reactivity and DT relate differently to negative behaviors (e.g., negative response to emotional films, and smoking). The studies that do exist seem methodologically weak, especially as compared to the number of studies supporting emotional reactivity as an individual difference factor. Therefore, this study examined emotional reactivity as an individual difference factor in DT in relation to self-damaging behaviors.

\section{Learned Helplessness Overview}

\section{History and Definition of Learned Helplessness}

As summarized by Eisenberger et al. (1976), learned helplessness occurs when "a person or animal learns he 'is helpless with respect to some outcome when the outcome occurs independently of all his voluntary responses"' (Seligman, 1975, as cited on p. 227). Learned helplessness was initially studied by exposing dogs to electric shocks (O'Donnell, 2006). Dogs who were continuously exposed to an inescapable electric shock eventually stopped escape attempts (helplessness training). For, the dogs learned that they were not able to control the shock (Peterson, Maier, \& Seligman, 1993). Then, when 
placed into a new scenario in which the electric shock was avoidable through simple behaviors, dogs who had first experienced the helplessness training failed to learn to escape. Whereas, dogs who had not previously experienced the helplessness training did learn to escape. This phenomenon was termed learned helplessness ("Learned helplessness," 2007).

Later, the study of learned helplessness was extended to humans. Typically participants were first exposed to an aversive stimulus, such as an inescapable loud sound or an unsolvable math problem. Similarly to the phenomenon observed with dogs, the participants eventually stopped escape or solving attempts (helplessness training). Then, the participants were exposed to a new situation in which the aversive stimulus was avoidable or solvable. In some situations, the participants who has been exposed to helplessness training failed to learn to escape or learn to solve in the new situation, thus demonstrating the learned helplessness phenomenon.

However, whether or not the learned helplessness is generalized from one situation to another situation is more complex in humans than in dogs. According to attribution theory (as formulated in Peterson et al., 1993), whether or not learned helplessness is applied to new situations is based on the causal attributions individuals make about the reasons the outcome was noncontingent on their behaviors (O'Donnell, 2006). Learned helplessness is most likely to be generalized to new situations when the causal attribution is stable (the cause of the noncontingency will continue) and global (the cause of the noncontingency applies to all situations; Peterson, 2010). An important implication of the cognitive emphasis in attribution theory is that, "a person need only expect that an outcome is noncontingent for learned helplessness to result" (O'Donnell, 
2006, p. 778). Attributional styles which reflect learned helplessness have been linked to the development of a variety of deleterious results and psychological disorders including anger, anxiety, athletic failure, burnout, depression, fear, longer involuntary unemployment, low self-esteem, low academic performance, and poor adjustment to aging and severe illness (Peterson et al., 1993).

As originally formulated, learned helplessness theory proposed that individuals learn a lack of control when repeatedly exposed to aversive situations from which they cannot escape. More recently, studies in neuroscience have instead suggested that beliefs of lack of control might be the baseline response to uncontrollable aversive situations (Maier \& Seligman, 2016). If these results are upheld by future research, learned helplessness might best be characterized as an individual failing to learn that he/she has control over outcomes, rather than as an individual learning that he/she lacks control over outcomes. Either way, the result is an individual believing that outcomes are independent of his/her behavior (Eisenberger et al., 1976). Thus, learned helplessness is defined in this study as the extent to which an individual believes that outcomes are independent of his/her behavior.

In the context of emotional DT, learned helplessness can emerge in response to several conditions, of which I discuss three. First, learned helplessness may result from noncontingent, positive punishment for any tolerance or non-tolerance of distress. For example, a child who receives criticism from a parent regardless of the child's response to distress. The child learns that no matter how he/she responds to emotional distress, the response of the parent will be negative. Second, learned helplessness may result from noncontingent, positive reinforcement for any tolerance or non-tolerance of emotion. For 
example, a child who receives positive reinforcement from a parent regardless of his/her response to distress. The child learns that no matter how he/she responds to emotional distress, the response of the parent will be positive. Both of these scenarios can result in the belief that "No matter what I do in response to emotional distress, I have no control over the outcome." Third, learned helplessness may result from positive reinforcement of low effort behaviors, avoidance behaviors, or escape behaviors. For example, a child who receives praise from a parent for suppressing emotional distress or attention for engaging in NSSI in response to emotional distress. The child learns that it is best to engage in loweffort, avoidance, or escape strategies in response to emotional distress. The message in these three situations is, "It is better for me not to try to deal with the emotional distress" leading to the belief that personal agency is of no use (i.e., a state of learned helplessness).

\section{Learned Industriousness-Helplessness Continuum}

As indicated above, Lynch and Mizon (2011) proposed that low learned industriousness is an individual difference factor influencing low DT. In the context of this study, high learned helplessness is evaluated instead of low learned industriousness. That is, I conceptualize high learned helplessness as involving even less belief in personal agency than low learned industriousness. This conceptualization implies the presence of a continuum of learned agency ranging from low agency beliefs in learned helplessness to high agency beliefs in learned industriousness.

This continuum is suggested to stretch from "high-learned industriousness at one extreme, through decreasing industriousness until a neutral point were reached, to lowlearned helplessness and finally to high-learned helplessness at the other extreme" 
(Eisenberger et al., 1976, p. 228). For example, one of the items on the Learned Helplessness Scale (LHS; Quinless \& Nelson, 1988) asks: "No matter how much energy I put into a task, I feel I have no control over the outcome.” An individual who indicates "strongly agree" to this item would be considered to have high learned helplessness, since the individual expresses a low belief in personal control. Whereas, an individual who indicates "strongly disagree" to this item would be considered to have high learned industriousness, since the individual expresses a high belief in personal control. Thus, in terms of level of belief in personal agency, learned helplessness is the inverse of learned industriousness.

Of note, a continuum between learned helplessness and learned industriousness applies to level of belief in agency, but does not necessarily apply to the number of actions an individual will take in an attempt to control or change a situation. An individual with high learned helplessness is expected to take no action or engage in low effort actions in difficult situations. For example, a student with high learned helplessness when faced with a difficult math test may leave all the answers blank (non-action) or guess multiple-choice answers at random (low effort). However, though an individual with high learned industriousness is expected to be willing to try many different responses if needed to improve a situation, the individual may only need to take one action if an effective action has been previously learned. In the previous example, a student with high learned industriousness when faced with a difficult math test may make repeated efforts to manually solve a math problem (many high effort actions) or may apply a solving strategy they previously learned was effective (one high effort action). 
The presence of a continuum from learned helplessness to learned industriousness was evaluated historically, with mixed results, due to the difficulty of creating experiments without floor or ceiling effects. First I look at a study by Hiroto (1974), which demonstrated the presence of learned helplessness, but failed to demonstrate learned industriousness. Then, I look at a study by Eisenberger et al. (1976), which demonstrated the presence of learned industriousness, but failed to demonstrate learned helplessness.

\section{Evidence Supporting Learned Helplessness}

Hiroto (1974) examined the construct of learned helplessness among undergraduate students $(N=96)$ by utilizing a punishment schedule. To do so, he first exposed non-control participants to a pretreatment task. This apparatus in the pretreatment task consisted of a spring-loaded button in a small, wooden base. In the escapable group, students experienced a pretreatment in which the loud sound could be silenced by pressing the button. In the inescapable group, students experienced a pretreatment in which the loud sound could not be silenced by pressing the button. In the control group, no pretreatment was administered. Then, each of the groups was exposed to an apparatus in the treatment tasks which was "distinctively different" from the apparatus in the pretreatment task (Hiroto, 1974, p. 188). The treatment task was a Manipulandum Type S task (Turner \& Solomon, 1962, as cited on p. 188) which required the participant to move a sliding knob along a channel away from center. In each trial, moving the sliding knob in one direction would silence the loud sound. The direction in which the knob must be moved in order to silence the loud sound alternated between trials. 
Consistent with learned helplessness, the students who experienced an inescapable pretreatment demonstrated less escape-avoidance behavior on the treatment task than the other two groups $(F(2,84)=22.57, p<.01)$. This makes sense from the perspective of agency, since the students presumably learned that the loud sound was not contingent on their behavior in the pretreatment task, which they generalized to the treatment task. Yet, the students who experienced an escapable pretreatment did not differ from the control group in level of escape-avoidance behavior (sliding the knob) on the treatment task. Thus, learned industriousness was not observed. From the perspective of agency, students who had learned during the pretreatment task that the loud sound was contingent on their behavior should have made more attempts to discontinue the sound than controls. Similar results were achieved by Hiroto and Seligman (1975) with undergraduate students $(N=96,53 \%$ male). However, because the tasks in these studies were acquired quickly by control subjects, a ceiling effect may have resulted in the lack of observed learned industriousness effect (Eisenberger et al., 1976). Given that there was a 50\% chance of sliding the knob in the correct direction, this experiment presumably allowed controls to quickly learn that the sound discontinuation was contingent upon their actions.

\section{Evidence Supporting Learned Industriousness}

In response to the studies noted above, Eisenberger et al. (1976) examined the construct of learned industriousness among a group of second- and third-grade students $(N=144)$ by utilizing a reinforcement schedule. Both the training task and the test task were pictorial stimulus tasks which required the children to select one stimulus picture, 
from four stimuli pictures presented on a page. In order to minimize the possibility of a ceiling effect masking the learned industriousness effect, the tasks were designed to be acquired slowly by the control group. To minimize the risk of a training effect, the students were reinforced based on the content of the stimuli in the training task, but they were reinforced based on the arrangement of the stimuli in the test task.

The students were compared in paired tetrads of training conditions. In the narrow-range group, the child received contingent reinforcement for only one of the four groups of stimuli. In the broad-range random-trial group, the child received contingent reinforcement for all four groups of stimuli in a random distribution. (Broad contingent reinforcement is essentially the same as noncontingent reinforcement, because when nearly any emitted behavior is reinforced then it essentially becomes noncontingent - that is, the only contingency is that a behavior is emitted, but what behavior does not really matter). In the broad-range yoked-trial group, the child received reinforcement for all four groups of stimuli in the same distribution as their paired child in the narrow-range condition. In the control group, the child received the same number of trials as their paired narrow-range child, but without reinforcement comments (either in the presence of an experimenter in the task control group or without the presence of an experimenter in the isolation control group). In the test task, all children were reinforced for the position of the quadrant they selected, following their first selected quadrant (for example, if the participant choose the stimuli in quadrant 1 on the first two trials, but then selected quadrant 2 on the third trial, quadrant 2 was reinforced for the duration of the task).

Consistent with the prediction of a learned industriousness-helplessness continuum, the children in the narrow-range group reached discontinue criterion (defined 
as 6 consecutively correct responses) on the test task in fewer trials $(M=36.8)$ than all of the other groups (task control: $M=63.6$; isolation control: $M=77.7$ ). This supports the presence of a learned industriousness effect when stimuli receive contingent reinforcement. This makes sense from the perspective of agency, since the students presumably learned that praise was contingent on their behavior in the training task, which they generalized to the test task. However, the broad-range groups did not take more trials (random trial: $M=57.1$; yoked trial: $M=58.0$ ) to reach the discontinue criterion than the control groups. Thus, a learned helplessness effect was not identified when a broad range of stimuli received contingent reinforcement (in other words, noncontingent reinforcement). From the perspective of agency, students who had learned during the training task that praise was not contingent on their behavior should have taken more trials to reach discontinue criterion than the control groups; but, that was not found.

In terms of implications beyond this study, this result may indicate that the learned helplessness effect does not exist. However, given the breadth of research supporting the learned helplessness effect, this result may instead be due to the range of stimuli (four classes) not being broad enough to produce a learned helplessness effect in a reinforcement paradigm. Or, it may be due to a floor effect, since the task was intentionally designed to be acquired slowly by the control group.

\section{Learned Industriousness-Helplessness Continuum Summary}

Extensive review of the extant literature failed to turn up a study that effectively demonstrated the presence of learned helplessness and learned industriousness in the 
same study. However, this may be due to the competing impact of floor and ceiling effects in study design. Future research to confirm the presence of the learned industriousness-helplessness continuum is needed. However, evidence supports both directions of the continuum individually.

As discussed above, Lynch and Mizon (2011) proposed learned industriousness as an individual difference factor in low DT. They noted that low learned industriousness develops from a learning environment in which an individual is rewarded for low effort or avoidance/escape actions. Whereas, high learned industriousness develops from a learning environment in which an individual is rewarded for high-effort actions. In their model, when paired with high emotional reactivity, low learned industriousness is associated with low DT and high learned industriousness is associated with distress overtolerance. Whether considered from the lens of willingness to exert effort or the lens of belief in personal agency, learned industriousness makes theoretical sense as a possible individual difference factor in DT (since both are presumably associated with level of task persistence). However, if a learned industriousness-helplessness continuum does exist, then assessing low learned industriousness may not truly capture an individual's belief that outcomes are not contingent on their own actions. Rather, low industriousness would capture a point nearer to neutral, in which an individual has mixed beliefs about their own agency. So, I look at learned helplessness in this study, rather than low learned industriousness as originally proposed by Lynch and Mizon. Thus, I hypothesized that high learned helplessness would be associated with low DT.

In the following sections, I outline evidence supporting and opposing learned helplessness as an individual difference factor in DT. Research theoretically and 
empirically linking learned helplessness with DT is lacking. However, a few studies of related concepts, such as pain tolerance and emotional regulation, are examined to provide context. Further research is needed to directly examine the relationship between DT and learned helplessness.

\section{Evidence Supporting Learned Helplessness as an Individual Difference Factor in DT}

As indicated above, Lynch and Mizon (2011) suggested that learned industriousness is an individual difference factor in DT. Lejuez, Banducci, and Long (2013) also drew a connection between learned industriousness and distress tolerance, since both concepts have been developed in attempts to explain motivation for goal achievement. In support of the relationship, I look at the relationship between learned helplessness and physical pain tolerance as well as the relationship between learned helplessness and emotion regulation.

\section{Learned Helplessness and Physical Pain Tolerance}

Yamamoto et al. (2010) examined psychological characteristics of Japanese individuals with non-malignant chronic pain $(N=48,71 \%$ female, mean age $=43)$ through the administration of the Rorschach Comprehensive System. Results indicated that a greater percentage of individuals with chronic pain experienced "unusually high levels of distress and/or emotional discomfort" than individuals without chronic pain (chronic pain: $35 \%$, control: $20 \%, p<.05$ ). Individuals with chronic pain also experienced "a sense of helplessness due to situational stress" (chronic pain: $M=1.42$, $\mathrm{SD}=2.07$; control: $M=0.55, \mathrm{SD}=0.84, p<.01)$ with greater frequency than individuals 
without chronic pain (Yamamoto et al., 2010, p. 6). Thus, it appears that helplessness and high distress co-occurred in a chronic pain population.

However, this study spoke only to a greater likelihood of reporting distress, not necessarily low DT. For, it is possible that chronic pain elicits more emotional distress regardless of DT level. Also, these themes were drawn from projective assessment, rather than from self-report, so results may differ from self-report of DT and helplessness. Lastly, the co-occurrence of high emotional distress and helplessness does not necessitate a relationship between them, since the co-occurrence may be the result of a third variable. So, this study should be interpreted with great caution in the context of this review.

\section{Emotion Regulation and Helplessness in NSSI}

Slee et al. (2008) examined the role of emotion regulation strategies and suicidal cognitions in NSSI. The study compared a clinical group of Dutch women admitted to a hospital following an instance of NSSI $(N=85$, mean age $=24)$ to a control group of female vocational students without a history of $\operatorname{NSSI}(N=93$, mean age $=23)$. Indeed, the clinical group reported greater difficulties than the control group with emotion regulation including lack of awareness of emotional responses $(F=24.41, p<.01, F c o v$ $=7.40, p<.05, d=0.75)$, lack of clarity of emotional responses $(F=225.09, p<.01$, Fcov $=22.00, p<.01, d=2.28$ ), nonacceptance of emotional responses (similar to sDTS: Appraisal, $F=268.38, p<.01, F c o v=30.67, p<.01, d=2.44)$, limited access to emotion regulation strategies $(F=265.46, p<.01, F \operatorname{cov}=75.35, p<.01, d=2.49)$, difficulties controlling impulses when experiencing negative emotions $(F=293.73, p<$ $.01, F \operatorname{cov}=34.67, p<.01, d=2.59)$, and difficulties engaging in goal-directed behaviors 
when experiencing negative emotions (similar to sDTS: Absorption, $F=178.94, p<.01$, $F c o v=24.49, p<.01, d=1.99)$. These differences held true after controlling for differences in depression severity (as indicated by the Fcov values). Also, membership in the clinical group was correlated with significantly greater levels of perceived burdensomeness $(r=.80)$, helplessness $(r=.76)$, low DT $(r=.82)$, and unlovability $(r=$ .78) on the Suicide Cognition Scale (Rudd et al., 2001 as cited in Slee et al., 2008, p. 277). Additionally, depressive symptoms $(B=.44$, Wald $=6.19, p=.013)$, suicidal cognitions $(B=.60$, Wald $=3.97, p=.046)$, and nonacceptance of emotional responses $(B=.44$, Wald $=3.10, p=.078$, considered by the authors to be a significant $p$ value $)$ together predicted $73 \%$ of the variance in group membership $\left(\chi^{2}=226.82, p<.001\right)$ and correctly classifying $98 \%$ of the cases.

The correlation between low DT and helplessness alone was not listed. However, the authors noted that the correlations between subscales of the Suicide Cognition Scale ranged from .85 to .92 , so the correlation was high and within that range. This study thus demonstrated that self-reported difficulties with emotion regulation, helplessness, and low DT all co-occurred in a population of inpatients with NSSI. However, this study does not speak to the relationship between helplessness and DT, since the co-occurrence may be due to a third variable.

\section{Evidence Opposing Learned Helplessness as an Individual Difference Factor in DT}

\section{Learned Helplessness and Physical Pain Tolerance}

H. Feldman (1986) examined the relationship between attributional style and distress pain ratings among men $(N=101,87 \%$ Caucasian, mean age $=31)$. Attributional 
style was assessed by survey, with attributional style divided into positive internal attribution style (associated with learned industriousness) and negative external attribution style (associated with learned helplessness). Ratings of physical pain distress were gathered after the immersion of a hand into cold water. Results indicated that there was not a relationship between attribution style and distress pain ratings ( $r$ 's $=-.001$ to $.060, n s)$. Thus, it appears that learned helplessness was not associated with physical pain tolerance. However, it must be noted that attribution style, not learned helplessness, was assessed. Additionally, physical pain tolerance is thought to be most similar to DI rather than DT. So, the same pattern of results may not be found in emotional DT.

\section{DT and Negative Reinforcement}

Research has suggested that individuals with low DT are especially prone to engaging in negatively reinforcing avoidance behaviors, such as substance use (Brown et al., 2009), compulsive acquisition (Williams, 2012), or NSSI (Chapman \& DixonGordon, 2007). Macatee and Cougle (2015) suggested that low DT is "primarily reflective of negative affective responses to distress and strong action-tendencies to seek immediate negative reinforcement" (p. 37). Behavioral DT tasks have even been used as a proxy for engagement in negative reinforcement behavior (Daughters et al., 2009). On the surface, this research runs contrary to the theory of learned helplessness which anticipates that individuals will not expend effort toward escape and avoidance behaviors, since they have previously learned that they lack agency over the outcomes they experience.

Yet, individuals with high learned helplessness are expected to expend minimal effort toward reinforcement. As indicated above, this sometimes means expending no 
effort toward escape or avoidance behaviors. However, when there is a choice between a low-effort immediate negative reinforcer or a high-effort negative reinforcer that requires time, an individual with high learned helplessness is expected to take the less-effortful route. The negatively reinforcing avoidance behaviors with which low DT is associated are immediate. In fact, Trafton and Gifford (2011) described low distress tolerance as "the propensity to respond to immediate (negative) reinforcement or reward, as opposed to pursuing alternate reinforcers that may become accessible when immediate negative reinforcement is inhibited or not pursued" (as summarized in Leyro et al., 2010, p. 578).

If, in fact, learned helplessness is a contributing factor to low DT, it may be that immediate negatively reinforcing behaviors are especially appealing to individuals with low DT because of the beliefs that they cannot have a long-term impact on their level of emotional distress. For, the use of avoidance strategies is more likely when an individual lacks access to other DT strategies (McHugh et al., 2013). Thus, individuals with low DT would opt for either inaction or actions with immediate negative reinforcement, rather than actions which require high-effort and time before negative reinforcement occurs (e.g. distress tolerance skills).

The directionality of the relationship between DT and engaging in negative reinforcement behaviors is unknown. One option is that low DT results in an individual being more vulnerable to engaging in negative reinforcement behaviors (Williams, 2012). Another option is that participating in negative reinforcement behaviors lowers an individual's DT level because the individual practices avoiding or escaping distressing emotions (Williams, 2012). A third option, embraced in this study, is that one's belief that he/she has no influence on his/her experienced distress (high learned helplessness) 
contributes to low tolerance of emotional distress (low DT), which contributes to an individual seeking self-damaging behaviors that may provide immediate negative reinforcement rather than emotionally healthy behaviors which may provide negative reinforcement in time.

\section{Learned Helplessness Summary}

In summary, learned helplessness is a factor conceptualized as involving low or no belief in personal agency. High learned helplessness interferes with an individual's motivation to work out a solution that terminates unfavorable conditions and to seek positive rewards (both of which may contribute to harmful behaviors and psychological disorders). There are no known studies that directly examined learned helplessness in relation to DT, so results with related constructs were reviewed. Results regarding the relationship between learned helplessness and physical pain tolerance are mixed. However, high helplessness occurred together with low DT in a sample of individuals with NSSI. Additionally, learned helplessness has been theoretically linked to an individual opting for actions with immediate negative reinforcement (e.g., NSSI) despite long-term harm, rather than opting for actions which require high-effort and time before negative reinforcement occurs (e.g., distress tolerance skills). Therefore, this study explored learned helplessness as an individual difference factor in DT in relation to selfdamaging behaviors.

\section{Relationship Between DT and Covariates}

The existing literature demonstrates associations between anxiety and depression and DT. In order to draw out the role of emotional reactivity and learned helplessness in 
DT and self-damaging behaviors, anxiety and depression were included as covariates in my study. Below, I outline what is known about the relations between DT and both anxiety and depression.

\section{DT and Anxiety}

The existing literature demonstrates a link between DT and a variety of anxiety symptoms (see Appendix A for a review). The nature of the link varies depending on the way in which DT is measured, the population sampled, and the anxiety symptom measured. I review the role of DT in AS, health anxiety, obsessive compulsive anxiety, panic, social anxiety, trait anxiety, and worry.

\section{DT and AS}

First, self-reported DT is negatively correlated with AS among undergraduate students (Norr et al., 2013), outpatients (Capron, Norr, Macatee, \& Schmidt, 2013), and community members (Johnson, Berenz, \& Zvolensky, 2012). Johnson et al. (2012) further found that self-reported DT predicted variance in total AS (an additional 14.1\% of variance), physical AS (with panic attack history accounted for an additional $8 \%$ of the variance), cognitive AS (an additional 13.9\% of the variance), and social AS (an additional $16.2 \%$ of the variance) after accounting for neuroticism, sex, and panic attack history. Yet, in the same study, behaviorally-assessed physical distress tolerance (as measured by the Breath Holding Duration [BHD] task) was not correlated with or predictive of AS. Keough, Riccardi, Timpano, Mitchell, and Schmidt (2010) found that although both DT and AS were individually predictive of variance in worry, social 
anxiety, and obsessive compulsive symptoms, DT and AS did not interact in the prediction of the symptoms.

\section{DT and Health Anxiety}

Second, in a study of a nonclinical sample Fergus, Bardeen, and Orcutt (2015) found self-reported DT to be negatively correlated with health anxiety (in the study, the sDTS score was reversed such that it was a positive correlation). They also found DT to be uniquely predictive of health anxiety while also considering IU, TA, FT, and DI (together, DT, IU, and DI explained $63 \%$ of the variance). However, IU and DI accounted for greater variance in health anxiety than DT. Intolerance of uncertainty and DI were also negatively correlated with DT (Norr et al., 2013).

\section{DT and Obsessive Compulsive Anxiety}

Third, DT has been found to be negatively correlated with obsessive compulsive symptoms among undergraduate students when hoarding symptoms are included (Keough et al., 2010) and excluded (Norr et al., 2013). Norr et al. (2013) found that DT was not predictive of obsessive compulsive symptoms excluding hoarding symptoms when controlling for negative affect, sex, AS, and IU. Yet, Keough et al. (2010) found that DT was predictive of obsessive compulsive symptoms including hoarding symptoms when controlling for AS, general anxiety, and depression.

\section{DT and Panic}

Fourth, Keough et al. (2010) found DT to be negatively correlated with panic symptoms and predictive of panic symptoms when controlling for AS, general anxiety, and depression among undergraduate students. Kutz, Marshall, Bernstein, and Zvolensky 
(2010) found DT to be negatively correlated with post-biological challenge (carbon dioxide-enriched air challenge) panic attacks and cognitive panic symptoms, but not physical panic symptoms among nonclinical individuals. However, DT was not predictive of post-challenge panic attacks, cognitive panic symptoms, or physical panic symptoms when controlling for recent panic attack history, negative affect, and AS. Similarly, Leyro, Berenz, Brandt, Smits, and Zvolensky (2012) found that neither selfreported DT nor behaviorally-assessed DT (as measured by the Mirror-tracing Persistence Task, MTPT, Quinn, Brandon, \& Copeland, 1996) predicted panic attack symptoms after a biological challenge (carbon dioxide-enriched air challenge) when controlling for negative affect, sex, panic attack history, number of axis 1 diagnoses according to the Diagnostic and Statistical Manual of Mental Disorders (4th edition; American Psychiatric Association, 2000), perseveration, perfectionism, and persistence.

\section{DT and Social Anxiety}

Fifth, DT was found to be negatively correlated with social anxiety among undergraduate students (Keough et al., 2010; Norr et al., 2013). Keough et al. (2010) found that DT was predictive of social anxiety when controlling for AS, general anxiety, and depression. Yet, Norr et al. (2013) did not find DT to be predictive of social anxiety when controlling for negative affect, sex, AS, and IU in Study 1 or when controlling for trait anxiety, sex, AS, and IU in Study 2.

\section{DT and Trait Anxiety}

Sixth, self-reported DT is negatively correlated with trait anxiety (Norr et al., 2013) and generalized anxiety (Anestis, Bagge, Tull, \& Joiner, 2011; Bender, Anestis, 
Anestis, Gordon, \& Joiner, 2012; Huang, Szabó, \& Han, 2009; Keough et al., 2010)

among undergraduate students. However, in a study by Bender et al. (2012), behaviorally-assessed DT (as measured by the DTT) was not found to be correlated with general anxiety.

\section{DT and Worry}

Lastly, DT is negatively correlated with worry among undergraduate students (Keough et al., 2010; Norr et al., 2013), inpatients (Kertz, Stevens, McHugh, \& Björgvinsson, 2015) and outpatients (Macatee et al., 2015). Keough et al. (2010) found that DT was predictive of worry when controlling for AS, general anxiety, and depression. Norr et al. (2013) similarly found DT to be predictive of worry when controlling for negative affect, sex, AS, and IU in Study 1. However, they did not find DT to be predictive of worry when controlling for trait anxiety, sex, AS, and IU in Study 2. Macatee et al. (2015) found that outpatients with Generalized Anxiety Disorder diagnoses had significantly lower DT than nonclinical controls (study 1). They also found that, among undergraduates, baseline DT predicted worry one month later and daily worry reported during that interval. Among both undergraduate students and inpatients, Kertz et al. (2015) found that lower levels of DT (reported as the inverse, distress intolerance in the original study) were associated with higher levels of worry. In the clinical sample, the relationship between worry and DT was partially mediated by negative problem orientation and negative beliefs about worry. Whereas, in the student sample, the relationship was partially mediated by both positive and negative beliefs about worry. 


\section{DT and Depression}

The existing literature supports a relationship between DT and depression (see Appendix A for a review). Self-reported DT was negatively correlated with depression symptoms among undergraduate students (Anestis, Moberg, \& Arnau, 2014; Bender et al., 2012; Keough et al., 2010; Norr et al., 2014; Peterson, Davis-Becker, \& Fischer, 2014), outpatients (Allan, Macatee, Norr, \& Schmidt, 2014; Capron et al., 2013; Williams et al., 2013), inpatients receiving treatment for SUDs (Anestis, Knorr, Tull, Lavender, \& Gratz, 2013; Anestis, Pennings, et al., 2013) and nonclinical individuals meeting criteria for MDD (Williams et al., 2013). It is also negatively correlated with the diagnosis of MDD among outpatients (Williams et al., 2013). However, behaviorallyassessed DT was not correlated with depression among undergraduate students when measured by the DTT (Bender et al., 2012) or among a nonclinical sample when measured by the MTPT (Gratz et al., 2011). Thus, it appears that self-reported DT is negatively correlated with depression, but behaviorally-assessed DT is not. The relationship is further nuanced when accounting for the role of AS and the role of the DT subscales.

\section{DT, AS, and Depression}

Norr et al. (2014) examined the impact of a single psychoeducational group focused on AS reduction $(N=52)$ versus a single psychoeducational group focused on broad physical health education $(N=52)$ among undergraduate students with elevated anxiety drawn from a psychology research pool (84\% female, $82 \%$ Caucasian, mean age =19). Measures of DT, DI, and AS were collected pre-treatment and at one week posttreatment. Measures of anxiety and depression symptoms were collected pre-treatment 
and at one month post-treatment. Emotional DT was assessed using self-report (sDTS) and DI was assessed using self-report (Discomfort Intolerance Scale [DIS], Schmidt, Richey, \& Fitzpatrick, 2006).

Pre-intervention DT was negatively correlated with pre-intervention AS $(r=-.58$, $p<.05)$, DI $(r=-.24, p<.05)$, worry $(r=-.65, p<.05)$, anxiety $(r=-.58, p<.05)$, and depression $(r=-.57, p<.05)$ symptoms. For the experimental group, Week 1 data collection revealed increased DT $(\beta=.20, p<.001)$ and decreased AS $(\beta=-.41, p<$ $.001)$, but no changes were observed in DI $(\beta=-.07, n s)$. Week 1 DT fully mediated the relationship between the intervention and worry and depression symptoms at 1 month post-treatment and partially mediated the relationship with anxiety symptoms $\left(\chi^{2}=14.76\right.$, $p=.26, \mathrm{CFI}=.99, \mathrm{RMSEA}=.05)$. However, when AS was added as an additional mediation pathway, the mediation effect of DT was no longer significant $\left(\chi^{2}=19.51, p=\right.$ $.49, \mathrm{CFI}=1.00, \mathrm{RMSEA}=.00)$. This study lends support to the idea that AS is a lower order factor of DT, since an intervention which decreased AS also increased DT. However, though the AS intervention resulted in increased DT, increased DT was not the pathway to symptom improvement in this study.

An advantage of this study was the inclusion of both a control and an experimental group. The study was also strengthened by considering DT and AS both independently and concurrently. The study also used pre-intervention levels as covariates in post-treatment analyses. Shortcomings of the study included a largely female and Caucasian undergraduate sample and lack of data collection on the affect tolerances at 1 month post-treatment. Additionally, the impact of the intervention on depression 
scores was only marginal. Thus, it is unclear whether the patterns would hold up with a longer and/or more impactful intervention.

\section{DT Subscales and Depression}

Williams et al. (2013) examined the impact of 6 online CBT sessions over a 10week period on outpatients attending a sadness program who met probable criteria for diagnosis of depression $(N=75,65 \%$ female). Distress tolerance was measured by selfreport (sDTS) at pre- and post-treatment.

Results indicated that all sDTS subscales were negatively correlated with baseline depression $(r$ 's $=-.27$ to $-.40, p<.05)$ and psychological distress $(r$ 's $=-.27$ to $-.36, p<$ $.05)$. Scores on sDTS: Absorption $(t(47.71)=2.74, p<.01, d=0.28)$, sDTS: Appraisal $(t(44.49)=3.24, p<.01, d=0.39)$, and sDTS: Tolerance $(t(43.42)=3.18, p<.01, d=$ $0.32)$, but not sDTS: Regulation $(t(44.16)=0.64, n s, d=0.06)$, increased between preand post-treatment assessment. Both depression severity $(t(45.90)=7.28, p<.001, d=$ 1.02) and psychological distress $(t(44.82)=8.53, p<.001, d=1.14)$ decreased from preto post-treatment. Both time $(F(1,78.39)=7.90, p=.006)$ and total DT $(F(1,96.14)=$ $8.57, p=.004)$ were associated with post-treatment depression. Both time $(F(1,74.33)=$ $5.66, p=.02)$ and total DT $(F(1,99.42)=10.83, p=.001)$ were associated with posttreatment psychological distress. However, the interaction between time and total DT was not associated with post-treatment psychological distress or post-treatment depression. Individuals with low DT at baseline had higher baseline and post-treatment depression and psychological distress compared to individuals with high DT at baseline. This study suggests that an individual's level of DT prior to intervention was associated with the 
individual's level of depression and level of psychological distress following a CBT intervention.

The authors then replicated this study among participants recruited online $(N=$ $35,79 \%$ female, mean age $=42$ ), with half of the subjects receiving the intervention via smart phone. In this study, only sDTS: Tolerance and sDTS: Regulation were calculated. Results indicated both sDTS: Tolerance and sDTS: Regulation were again negatively correlated with baseline depression (both $r$ 's $=-.38, p<.05$ ) and psychological distress (sDTS: Tolerance: $r=-.42, p<.05$; sDTS: Regulation: $r=-.43, p<.05$ ). Time, and sDTS total score (sDTS: Tolerance and sDTS: Regulation) were each associated with post-treatment depression and psychological distress $\left(F^{\prime} \mathrm{s}(1,25.07-25.72)=9.76-9.81\right.$, $p$ 's < .01). Both sDTS: Tolerance $(M=2.16, S E=.20$ to $M=2.88, S E=.22, d=0.49)$ and sDTS: Regulation $(M=2.24, S E=.18$ to $M=2.75, S E=.20, d=0.63)$ increased from baseline to post-treatment.

An advantage of this study was that it accounted for all of the DT subscales in the first study, and two of the DT subscales in the second study. An advantage of the second study was the confirmation of a depression diagnosis, whereas in the first study a diagnosis was not confirmed. However, a shortcoming of the second study was a small sample size $(N=35)$. Both studies were weakened by the lack of a control group and the lack of extended DT data. Further, though these two studies demonstrated that depression severity decreased concurrently with DT increases, the studies do not demonstrate that the increase in DT mediated the decrease in depression. 


\section{DT and Covariates Summary}

In summary, research repeatedly (although not invariably) shows DT to be correlated with both anxiety and depression. Distress tolerance has also been shown to have a predictive role in various anxiety symptoms (e.g. AS, health anxiety, panic symptoms, and worry). Yet, anxiety and depression are typically separate factors from DT. Since anxiety and depression may relate to outcome variables in a similar manner as DT, this study included both anxiety and depression as covariates.

\section{Relationship Between Distress Tolerance and Self-Damaging Behaviors}

As indicated above, DT is thought to have transdiagnostic relevance, and has been studied in relation to many different diagnostic and clinical concerns (for a review see Leyro, 2010). For instance, DT has shown negative correlations with anxiety disorders (Keough et al., 2010), physical dating violence perpetration and victimization (Shorey et al., 2013), depression (Williams et al., 2013), early treatment dropout (Daughters et al., 2005), gambling severity (Lisle, Dowling, \& Allen, 2014), hoarding (Hezel \& Hooley, 2014), hurried driving (Beck et al., 2013), impulsive behaviors (Anestis et al., 2012), number of casual sex partners for individuals with attention-deficit/hyperactivity disorder (Van Eck, Flory, \& Willis, 2015), obsessions (Cougle, Timpano, Fitch, \& Hawkins, 2011), personality disorders (Daughters et al., 2008), schizophrenia (Chiappelli et al., 2014), substance dependence (Buckner, Keough, \& Schmidt, 2007), trait anger (Hawkins et al., 2013), and less post-traumatic growth following cancer (Baník \& Gajdošová, 2014). The focus of this study was on three types of self-damaging behaviors: disordered 
eating behaviors, NSSI, and suicidality. Existing literature regarding the relation between DT and these behaviors is reviewed next.

\section{DT and Eating Disorders}

The existing literature supports a relationship between DT and eating attitudes and patterns (see Appendix A for a review). On one end of the spectrum, DT is positively correlated with healthy eating patterns including intuitive eating and body image acceptance (Schoenefeld \& Webb, 2013) and interoceptive awareness (Anestis et al., 2007). On the other end of the spectrum, DT is negatively correlated with unhealthy eating attitudes and patterns (Raykos, Byrne, \& Watson, 2009) including body disordered eating attitudes (Kelly, Cotter, \& Mazzeo, 2014), bulimic symptoms (Anestis et al., 2007; Corstorphine et al., 2007; Lavender, Happel, Anestis, Tull, \& Gratz, 2015), body dissatisfaction (Anestis et al., 2007; Corstorphine et al., 2007), disinhibited eating (Lydecker, Hubbard, Tully, Utsey, \& Mazzeo, 2014), desire to be thinner (Anestis et al., 2007; Lydecker et al., 2014), and binge eating symptoms (Kenardy, Arnow, \& Agras, 1996). Yet, the relationship proves to be more nuanced when accounting for differences in DT subscales and differences in disordered eating behaviors.

\section{The Role of cDTS: Avoidance of Affect}

For instance, in a cross-sectional study, Corstorphine et al. (2007) compared females in inpatient treatment for $\operatorname{EDs}(N=72)$ to undergraduate and graduate females without a history of an $\mathrm{ED}(N=62)$. The healthy control group was matched to the ED group in age (mean age $=28)$ and body mass index (mean body mass index $=23)$. 
Participants completed a self-report measure of emotional DT, the cDTS, which was developed for the purpose of this study. The cDTS is composed of 20 items which ask the respondent to assess the extent to which he/she engages in the designated coping strategy from 1 (never) to 5 (all the time). The authors conducted factor analysis on the results of the whole sample and identified 14 items loading on to 3 subscales. First, cDTS: Anticipate and Distract assesses the extent to which the respondent anticipates negative affect and distracts him/herself from it with items such as "If I know I am going to be alone for any length of time I will make sure that I have lots of things to do to make the time pass quickly." Second, cDTS: Avoidance of Affect assesses the extent to which the respondent avoids situations which trigger negative affect with items such as "I won't engage in activities/relationships about which I know I will become too enthusiastic."

Third, cDTS: Accept and Manage assesses the extent to which the respondent accepts and uses appropriate behaviors to manage distress with items such as "If I find I am getting too anxious, I will do something to soothe myself (e.g., listen to music, read a book)." Participants also completed a self-report measure of eating pathology which produced a global score composed of drive for thinness, bulimia, and body dissatisfaction subscales. After the completion of factor analysis, Corstorphine et al. (2007) compared the cDTS scores between the clinical and control groups. Results indicated that the clinical group scored higher than the control group on cDTS: Avoidance of Affect $(t=5.68, p=$ .001). The clinical group scored lower than the control group on cDTS: Accept and Manage ( $t=2.25, p=.026)$. The clinical and control groups did not differ on cDTS: Anticipate and Distract $(t=1.36, p=.175)$. In the control group, bulimia symptoms were positively correlated with cDTS: Avoidance of Affect $(r=.379, p<.01)$. In the clinical 
group, body dissatisfaction symptoms were positively correlated with cDTS: Avoidance of Affect $(r=.259, p<.05)$ and negatively correlated with cDTS: Accept and Manage $(r$ $=-.238, p<.05)$.

An advantage of this study was the use of both a clinical and a control group. Also, the groups were matched on age and body mass index. This study also included consideration of subscales of the cDTS. However, a shortcoming of this study was the use of the same sample for EFA and group comparisons. The results would be strengthened by CFA using a separate sample. Further, the sample was limited to females, so the results may not be generalizable to males. Overall, this study suggests that individuals with EDs report more cDTS: Avoidance of Affect and lower cDTS: Accept and Manage.

\section{The Role of cDTS: Behavioral Avoidance of Positive Affect}

The relationship between cDTS: Avoidance of Affect and eating pathology was further clarified by Raykos et al. (2009) who conducted CFA on the cDTS subscales identified in the original factor analysis described above (Corstorphine et al., 2007). Raykos et al. (2009) found the original subscales to be a poor fit to their sample of female outpatients at an ED clinic. So, they conducted EFA. Their sample was composed of females meeting diagnostic criteria for $\mathrm{AN}(N=29$; mean age $=24)$, females meeting diagnostic criteria for bulimia nervosa $(N=85$, mean age $=27)$, and females meeting diagnostic criteria for eating disorder not otherwise specified $(N=90$, mean age $=27)$.

Their EFA revealed 13 items loading onto four subscales. First, cDTS:

Anticipating and Managing Affect measures the extent to which respondents both 
anticipate negative affect and use behaviors to manage it with items such as "If I am feeling anxious, I will do something practical to steady my nerves (e.g., clean the house)." Second, cDTS: Anticipating and Managing Loneliness measures the extent to which respondents both anticipate loneliness and use behaviors to manage it with items such as "When I am lonely, I call a friend or find someone to keep me company." Third, cDTS: Cognitive Avoidance of Affect measures the extent to which respondents attempt to manage affect by blocking thoughts with items such as "I tend to avoid thinking about the things that frustrate me." Lastly, cDTS: Behavioral Avoidance of Positive Affect measures the extent to which respondents avoid situations which trigger positive affect with items such as "If I find myself enjoying something too much, I will avoid them."

Of these subscales, results indicated that cDTS: Behavioral Avoidance of Positive Affect was positively correlated with global eating pathology $(r=.35, p<.001)$, restraint $(r=.20, p<.01)$, eating concern $(r=.34, p<.001)$, weight concern $(r=.35, p<.001)$, and shape concern $(r=.30, p<.001)$. The other three subscales (cDTS: Cognitive Avoidance of Affect, cDTS: Anticipating and Managing Loneliness, and cDTS: Anticipating and Managing Affect) were not correlated with the same measures ( $r$ 's $=-.06$ to $.11, n s)$. Except, cDTS: Anticipating and Managing Affect was positively correlated with weight concern $(r=.16, p<.05)$.

An advantage of this study was the use of a clinical sample. However, a shortcoming of the study was the use of the same sample for EFA and correlation analysis. The results would be strengthened by CFA using a separate sample. Additionally, the use of an entirely female sample does not allow for generalization of these results to men. Overall, this study suggests that in a clinical sample the relationship 
between eating pathology and avoidance of affect is more specifically a relationship between eating pathology and behavioral attempts to avoid positive affect.

In a cross-sectional study, Lampard, Byrne, McLean, and Fursland (2011) conducted CFA on the subscale divisions of the cDTS identified by both Corstorphine et al. (2007) and Raykos et al. (2009), as described above. The sample included a nonclinical sample of undergraduate females $(N=227$, mean age $=21)$ and female outpatients receiving treatment at an ED clinic $(N=257$, mean age $=26)$. Of note, the sample used in the study by Raykos et al. (2009) formed a subset of the clinical sample in the present study. Results of the confirmatory factor analyses in the full sample indicated that neither of the prior subscale models were a good fit for this sample. As such, EFA was conducted in the nonclinical undergraduate sample on nine items pertaining to avoidance of affect.

Their EFA revealed eight items loading onto three subscales. First, cDTS:

Behavioral Avoidance of Positive Affect measures the extent to which respondents avoid situations which trigger positive affect with items such as "I won't engage in activities/relationships about which I know I will become too enthusiastic.” This subscale's composition is identical to that identified by Raykos et al. (2009). Second, cDTS: Cognitive Avoidance of Affect measures the extent to which respondents attempt to manage affect by blocking thoughts with items such as "I tend to avoid thinking about the things that frustrate me." This subscale's composition differed from that identified by Raykos et al. (2009) by one question. Third, cDTS: Behavioral Avoidance of Negative Affect measures the extent to which respondents avoid situations which trigger negative affect with items such as "I avoid situations that I know will make me nervous." 
The authors then tested the above EFA by conducting CFA among the clinical sample. Indeed, the subscale model was a good fit for the sample $\left(\chi^{2}=28.03, p=.04\right.$, RMSEA $=.05$, SRMR $=.06, \mathrm{GFI}=.95, \mathrm{CFI}=.98) . \mathrm{cDTS}$ : Behavioral Avoidance of Positive Affect (clinical: $r=.37, p<.001$; nonclinical: $r=.17, p<.05$ ) and cDTS: Behavioral Avoidance of Negative Affect (clinical: $r=.20, p<.01$; nonclinical: $r=.21, p$ $<.01)$ were positively correlated with general eating pathology in both the clinical and nonclinical sample. cDTS: Cognitive Avoidance of Affect was negatively correlated ( $r=$ $.15, p<.05)$ with general eating pathology in the nonclinical sample only. The clinical sample reported greater cDTS: Behavioral Avoidance of Positive Affect $(t(482)=3.56, p$ $<.001)$ and lower cDTS: Cognitive Avoidance of Affect $(t(482)=3.46, p<.01)$ than the nonclinical group. No group difference was observed on cDTS: Behavioral Avoidance of Negative Affect $(t(482)=0.24, n s)$.

An advantage of this study was the use of both clinical and nonclinical groups. Additionally, this study used a different portion of the sample for CFA than was used for EFA. A shortcoming of this study was the use of an all-female sample. Results should be confirmed among a male sample to allow for these results to be generalized. Overall, this study built on Corstorphine et al. (2007)'s discovery of the role of cDTS: Avoidance of Affect and Raykos et al. (2009)'s discovery of the role of cDTS: Behavioral Avoidance of Positive Affect by confirming that individuals with EDs demonstrate greater cDTS: Behavioral Avoidance of Positive affect, but not greater cDTS: Behavioral Avoidance of Negative Affect compared to healthy controls. 


\section{The Role of DT in Anorexia Nervosa}

In a cross-sectional study, Oldershaw et al. (2012) compared outpatients currently diagnosed with $\mathrm{AN}(N=40$, mean age $=26)$ to individuals with a history of AN who had been without symptoms for at least a year $(N=24$, mean age $=28)$ and to individuals without a history of $\mathrm{AN}(N=48$, mean age $=28)$. The sample was drawn from the United Kingdom and was largely female (98\%). The Healthy Control group was matched to the Recovered AN group on age, gender, and intelligence quotient. The Recovered AN group was recruited from an online recovery community, whereas the Healthy Control group was recruited through advertisements and personal contacts. Participants completed a self-report measure of emotional DT, the cDTS. For this study, Oldershaw et al. (2012) analyzed the three cDTS subscales identified in the original factor analysis conducted by Corstorphine et al. (2007): cDTS: Anticipate and Distract, cDTS: Avoidance of Affect, and cDTS: Accept and Manage. Participants also completed a self-report measure of eating pathology which produced a global score composed of dietary restriction, eating concern, weight concern, and shape concern subscales.

Results indicated that cDTS: Avoidance of Affect was positively correlated with eating pathology in the overall sample $(r=.528, p<.001)$. However, cDTS: Anticipate and Distract $(r=.125, n s)$ and cDTS: Accept and Manage $(r=-.057, n s)$ were not correlated with eating pathology. Indeed, the Current AN group endorsed higher cDTS: Avoidance of Affect than both the Recovered AN ( $p=.003, d=0.79)$ and Healthy Control $(p<.001, d=0.98)$ groups. Yet, the Current AN group did not differ from the Recovered AN group or Healthy Control group on cDTS: Anticipate and Distract or cDTS: Accept and Manage. Further, the Recovered AN and Healthy Control groups did 
not differ from each other on any of the DT subscales. Overall, this study suggests that individuals with current AN demonstrate greater cDTS: Avoidance of Affect than individuals with prior $\mathrm{AN}$ and individuals with no history of AN.

An advantage of this study was the consideration of both current and recovered AN groups in comparison to a control group. Also, the recovered AN group and control group were matched on demographic variables. A shortcoming of the study was the use of relatively small sample sizes, particularly for the Recovered AN group. Also, the study utilized the original cDTS subscale divisions which have questionable validity (Lampard et al., 2011; Raykos et al., 2009). Additionally, the study used a largely female sample which limits generalizability to males. Further, the study was unable to account for depression and anxiety as covariates due to non-parametric data.

Of note, Hambrook et al. (2011) used the same data as the Oldershaw et al. (2012) study to compare the Current AN group with the Healthy Control group. The Current AN group and the Healthy Control group no longer differed on the cDTS: Avoidance of Affect scale after controlling for depression, anxiety, and age. Thus, in this sample, the relationship between eating pathology and cDTS: Avoidance of Affect was better accounted for by differences in depression, anxiety, and age.

\section{The Role of DT in Binge Eating and Overeating}

The relationship between DT and overeating symptoms and binge eating symptoms has been studied among undergraduate student samples. Kozak and Fought (2011) found that low DT (as measured by the sDTS) was associated with overeating as a way to cope with negative emotions, overeating in response to environmental food cues, 
and general overeating after controlling for sex and body mass index. Webb and Forman (2013) found that the relationship between positive self-compassion and binge eating severity was mediated by both unconditional self-acceptance and emotional tolerance (as measured by the Emotional Tolerance Scale [ETS], Kenardy et al., 1996 as cited on p. 225) after accounting for body mass index. Of note, the definition of emotional tolerance in the study focused specifically on the aversiveness of negative emotions associated with overeating.

In a cross-sectional study, Kelly et al. (2014) examined the role of both DT and negative urgency ("the tendency to act impulsively when distressed," p. 454) in binge eating frequency among undergraduate women $(N=186$; mean age $=19)$ who had participated in binge eating behavior within the last 28 days. Participants were excluded if they endorsed compensatory behavior more than 2 times a week or had a body mass index below 18.5. Participants were recruited from an introductory psychology course. Participants completed a self-report measure of emotional DT, the sDTS. In this study, only the global sDTS score was used. Participants also completed self-report measures of depression, impulsivity (lack of planning, lack of perseverance, negative urgency, and sensation seeking), disordered eating attitudes, and binge eating episode frequency. Sensation seeking is defined as "the tendency to seek out activities involving risk or thrill” (Bender et al., 2012, p. 81).

Results indicated that DT was negatively correlated with both disordered eating attitudes $(r=-.30, p<.001)$ and binge eating episode frequency $(r=-.24, p<.001)$. However, DT did not predict binge eating episodes after accounting for depression, race/ethnicity, body mass index, and impulsivity $(\beta=-.16, n s)$. Whereas, negative 
urgency did predict binge eating episodes after controlling for the same variables ( $\beta=$ $.28, p<.05$ ). However, neither DT nor negative urgency moderated (a) the association between depression symptoms and binge eating frequency or (b) the association between disordered eating attitudes and binge eating frequency. The study did not examine DT as a predictor of disordered eating attitudes. Thus, in this sample, DT was not predictive of binge eating frequency.

An advantage of this study was the consideration of both DT and impulsivity in the prediction of disordered eating attitudes and binge eating episode frequency. Additionally, the study accounted for race/ethnicity and body mass index. Also, the sample was relatively diverse in race/ethnicity (53.3\% White). A shortcoming of the study was the use of an entirely undergraduate and female sample, since that limits the generalizability of the findings. Further, the study did not account for the sDTS subscales, which may have highlighted differential patterns among sDTS subscales. Overall, DT, though negatively correlated with disordered eating attitudes and binge eating frequency, was not predictive of binge eating frequency after accounting for race/ethnicity, body mass index, and impulsivity.

\section{The Role of DT in Bulimia Nervosa}

As indicated above, Corstorphine et al. (2007) found that bulimic symptoms were positively correlated with cDTS: Avoidance of Affect in a nonclinical university sample. Anestis et al. (2007) similarly found that low DT predicted bulimic symptoms in a nonclinical university sample, after controlling for sex, depression, anxiety, AS, negative affect, urgency, sensation seeking, lack of premeditation, lack of perseverance, interoceptive awareness, perfectionism, drive for thinness, and body dissatisfaction. 
Lavender et al. (2015) extended these findings by examining the role of DT and Negative Affect Eating Expectancies (beliefs that eating will reduce negative affect) among a sample of inpatients receiving treatment for SUDs $(N=93,56 \%$ male, mean age =36) through a cross-sectional study. Participants completed a self-report measure of emotional DT, the sDTS. In this study, only the global sDTS score was used. Participants also completed self-report measures of bulimic symptoms, Negative Affect Eating Expectancies, and negative affect.

Results indicated that DT was negatively correlated with bulimic symptoms ( $r$ $=-.56, p<.001)$, negative affect $(r=-.54, p<.001)$, and Negative Affect Eating Expectancies $(r=-.32, p<.01)$. Further, DT $(t=-5.06, \beta=-.52, p<.001)$, Negative Affect Eating Expectancies $(t=2.84, \beta=.27, p=.006)$, and the interaction between DT and Negative Affect Eating Expectancies $(t=-2.21, \beta=-.20, p=.03)$ were predictive of bulimic symptoms, after controlling for sex and overall negative affect. The interaction was such that the magnitude of the association between DT and bulimic symptoms was greater at moderate levels of Negative Affect Eating Expectancies than at low levels. A nonsignificant trend continued this pattern between moderate and high levels of Negative Affect Eating Expectancies.

An advantage of this study was considering the roles of not only DT and Negative Eating Expectancies, but also the interaction between them. Additionally, the study controlled for sex and overall negative affect. However, the sample was not inpatient due to disordered eating. So, these results would have greater clinical generalizability if replicated in a clinical ED population. Overall, this study suggests that DT plays a 
predictive role in the severity of bulimic symptoms, particularly at higher levels of Negative Affect Eating Expectancies.

\section{DT and NSSI}

The existing literature supports a relationship between DT and NSSI (see Appendix A for a review). Self-reported emotional DT is negatively correlated with both NSSI lifetime frequency and with number of NSSI methods (Anestis, Kleiman, Lavender, Tull, \& Gratz, 2014; Anestis, Knorr, et al., 2013; Anestis, Pennings, et al., 2013; Peterson et al., 2014). Whereas, behaviorally-assessed emotional DT was not correlated with NSSI lifetime frequency (Gratz et al., 2011). However, women with a history of NSSI after exposure to an interpersonal stressor demonstrated less behaviorally-assessed emotional DT than women without a history of NSSI (Gratz et al., 2011). Additionally, an inpatient treatment group aimed at increasing DT skills successfully decreased NSSI frequency (Booth et al., 2014). Details of these studies are reviewed next.

\section{Role of DT in NSSI Lifetime Frequency}

NSSI lifetime frequency is sometimes used as a proxy for NSSI severity. Research has demonstrated a negative correlation between self-reported DT and NSSI lifetime frequency in a sample of inpatients receiving treatment for SUDs (Anestis, Knorr, et al., 2013; Anestis, Pennings, et al., 2013) as well as in a large samples of undergraduate students (Anestis, Kleiman, et al., 2014; Peterson et al., 2014). In a sample of undergraduate students in introductory psychology courses $(N=884 ; 78 \%$ female, 78\% Caucasian), Peterson et al. (2014) found that self-reported DT alone did not predict lifetime NSSI frequency, after controlling for sex, race, and age $(\beta=0.34, t=0.82, n s)$. 
However, there was a three-way interaction with DT, negative urgency, and depression predicting lifetime NSSI frequency $(\beta=-0.14, t=-2.74, p<.01)$. As such, individuals with low DT, high negative urgency, and high depression reported the highest lifetime NSSI frequency.

In a sample composed of individuals with at least one episode of NSSI within the past year $(N=42,70 \%$ female, $63 \%$ Caucasian, mean age $=19)$ and healthy controls $(N$ $=52,73 \%$ female, $71 \%$ Caucasian, mean age $=20)$, Gratz et al. (2011) found that behaviorally-assessed DT (as measured by latency to termination on MTPT-C) was not correlated with lifetime NSSI frequency $(p>.10)$. In this study, women with a history of NSSI who were exposed to an interpersonal stressor demonstrated less DT than women without a history of NSSI $\left(F_{1,86}=7.65, \eta_{\mathrm{p}}{ }^{2}=.08, p<.01\right)$. Women with a history of NSSI who were not exposed to an interpersonal stressor demonstrated greater DT than women without a history of NSSI. As such, it was only after exposure to an interpersonal stressor that women with a history of NSSI demonstrated low DT. Of note, the opposite pattern was observed among men. Thus, it appears that behaviorally-assessed DT plays a different role in NSSI lifetime frequency among women than among men.

\section{Role of DT in Number of NSSI Methods}

The number of NSSI methods used is also sometimes used as a proxy for NSSI severity. Among a sample of inpatients receiving treatment for SUDs $(N=93,55 \%$ male, $78 \%$ Caucasian, mean age $=36$ ), self-reported DT (as measured by the sDTS) was negatively correlated $(r=-.28, p<.01)$ with the number of NSSI methods used (Anestis, Knorr, et al., 2013; Anestis, Pennings, et al., 2013). 


\section{Evidence from Treatment Outcomes}

Evidence of the relationship between DT and NSSI also comes from treatment outcomes. Booth et al. (2014) conducted a group therapy intervention with inpatients with either a history of NSSI or strong ideation of NSSI $(N=114,80 \%$ female, mean age =35). All participants attended a skills training group for one hour a day, four days a week, for six weeks; the group focused on reducing NSSI. The group curriculum was adapted from DBT.

Participants completed a self-report of DT (sDTS). Participants also completed a measure of NSSI occurring within the past 6 weeks. Assessments were completed pretreatment, immediately post-treatment, and at 3 months post-treatment.

Results indicated that DT increased between pre-treatment $(M=6.32, S D=2.35)$ and post-treatment $(M=10.36, S D=3.68 ; p<.01)$ as well as between pre-treatment and 3 months post-treatment $(M=9.72, S D=4.33 ; p<.01)$. Similarly, NSSI frequency decreased between pre-treatment $(M=13.68, S D=21.81)$ and post-treatment $(M=4.50$, $S D=11.01 ; p=.01)$ as well as between pre-treatment and 3 months post-treatment $(M=$ 3.62, $S D=11.33 ; p=.01)$. In this study, a 6-week group intervention based on DBT resulted in increased DT and decreased NSSI both immediately post-treatment and at 3months post-treatment. Yet, it is unknown if the increase in DT was causative in the reduction of NSSI or simply concurrent with the reduction of NSSI.

An advantage of this study was the use of an inpatient group with an intervention protocol. It was also strengthened by the collection of data at 3 months post-treatment. However, a shortcoming of this study was the lack of a control group. Additionally, the study did not examine the relationship between DT and NSSI. Further, the study did not 
distinguish between suicidal and nonsuicidal self-injury, which blurs the impact of this study.

\section{DT and Suicide}

The existing literature reveals an inconsistent relationship between DT and suicidality (see Appendix A for a review). For instance, self-reported DT has been found to be predictive of the acquired capability for suicide (the capacity to enact lethal harm, which involves both decreased fear of death and increased tolerance of physical pain) (Anestis, Bender, Selby, Ribeiro, \& Joiner, 2011; Anestis, Moberg, et al., 2014), but not predictive of suicidal ideation (Capron et al., 2013). Self-reported DT has been found to be negatively correlated with lifetime suicide attempts in both inpatient (Anestis, Pennings, et al., 2013) and undergraduate samples (Anestis, Kleiman, et al., 2014), but not predictive of lifetime suicide attempts in a different undergraduate sample (Capron et al., 2013). Further, self-reported DT has been found to be associated with perceived burdensomeness and thwarted belongingness (Anestis, Bagge, et al., 2011; Anestis, Moberg, et al., 2014). However, a study by Anestis and Joiner (2012) did not find behaviorally-assessed DT to predict perceived burdensome and thwarted belongingness. These studies are described next.

\section{Role of DT in Suicidal Desire}

The interpersonal-psychological theory of suicidal behavior (Joiner, 2005, as cited in Anestis, Bender, et al., 2011, p. 172) suggests that high thwarted belongingness and perceived burdensomeness are important components in the development of suicidal desire. In cross-sectional studies with undergraduate students, Anestis, Bagge, et al. 
(2011) and Anestis, Moberg, et al. (2014) found self-reported DT to be negatively correlated with both thwarted belongingness and perceived burdensomeness. However, in the same sample of undergraduate students as Anestis, Bagge, et al. (2011), Anestis and Joiner (2012) found behaviorally-assessed DT to be unrelated to both thwarted belongingness and perceived burdensomeness. Thus, these studies suggest that selfreported and behaviorally-assessed DT function differently in perceived burdensomeness and thwarted belongingness.

High levels of trait hope have been associated with the acquired capability for suicide (Davidson, Wingate, Rasmussen, \& Slish, 2009; Davidson, Wingate, Slish, \& Rasmus, 2010). In a sample of undergraduate students $(N=220,77 \%$ female, $59 \%$ Caucasian, mean age $=21$ ), Anestis, Moberg, et al. (2014) found self-reported DT to be positively correlated with trait hope $(r=.41, p<.01)$. In fact, they found that DT fully mediated the predictive relationship between trait hope and acquired capability for suicide, after controlling for age, sex, income, and painful and provocative events. The authors noted that the findings align with an "emerging line of research pointing to DT as a central component of the capacity for lethal self-harm and may reflect that high levels of hope can, within certain contexts, serve as a less precise proxy measure of DT” (Anestis, Moberg, et al., 2014, p. 184).

\section{Role of DT in Pain Tolerance}

The interpersonal-psychological theory of suicidal behavior suggests that high physical pain tolerance is an important component of acquired capability for suicide (Joiner, 2005, as cited in Anestis, Moberg, et al., 2014). Anestis, Bagge, et al. (2011) found that self-reported DT (as measured by the sDTS) was predictive of pain tolerance 
(as measured by the Pressure Algometer Task, which measures an individual's tolerance of pressure administered to the second finger of their right hand) in an undergraduate sample $(N=283,59 \%$ female, $69 \%$ Caucasian, mean age $=19)$, such that individuals with low DT demonstrated lower pain tolerance than individuals with high DT after controlling for sensation seeking, lack of premeditation, lack of perseverance, depression, anxiety, and sex $(t=2.10, p<.04)$. In the same undergraduate sample, Bender et al. (2012) found that behaviorally-assessed DT (defined as latency to termination on the DTT) did not correlate $(r=.12, n s)$ with pain tolerance (as measured by the Pressure Algometer Task). However, DT did interact with sensation seeking to predict pain tolerance such that individuals with high DT and high sensation seeking demonstrated the highest pain tolerance while controlling for sex, negative urgency, lack of premeditation, lack of perseverance, depression, anxiety, perfectionism, and painful/provocative events $(t=2.22, p<.028)$. Thus, these studies suggest that both self-reported and behaviorallyassessed DT are predictive of physical pain tolerance.

\section{Role of DT in Suicidal Ideation}

In a study among outpatients at a university counseling center $(N=192,55 \%$ female, 70\% White, mean age $=38$ ), Capron et al. (2013) found that although selfreported DT (as measured by the sDTS) was negatively correlated with suicidal ideation experienced over the past two weeks $(r=-.42, p<.05)$, DT did not predict suicidal ideation after controlling for depression, sex, and AS $(t=1.63, \beta=.13, p=.10)$. Of note, the relationship trended toward significance, so it should be examined in a larger sample. 


\section{Role of DT in Past Suicide Attempts}

In a cross-sectional study among inpatients receiving treatment for SUDs $(N=93$, 55\% male, 77\% Caucasian, mean age $=36$ ), Anestis, Pennings, et al. (2013) found selfreported emotional DT (as measured by the sDTS) to be negatively correlated with the number of lifetime suicide attempts $(r=-.30, p<.01)$. In a study among undergraduate students $(N=1317,79 \%$ female, 55\% Caucasian, mean age $=21)$, Anestis, Kleiman, et al. (2014) similarly found a negative correlation $(r=-.27, p<.01)$ between self-reported emotional DT (as measured by the sDTS) and the number of lifetime suicide attempts. However, in a study among outpatients at a university counseling center $(N=192,55 \%$ female, 70\% Caucasian, mean age $=38$ ), Capron et al. (2013) found that self-reported DT (as measured by the sDTS) did not predict (Wald $=.81, p=.37$ ) the dichotomous likelihood of reporting a prior suicide attempt, after controlling for sex, depression, and AS.

\section{Role of DT in Suicide Potential}

In a study by Anestis, Knorr, et al. (2013), suicide potential was calculated based on the level of lethality of an individual's most lethal prior suicide attempt. Among a sample of inpatients receiving treatment for SUDs $(N=93,70 \%$ White, mean age $=36)$, self-reported DT (as measured by the sDTS) was negatively correlated with suicide potential $(r=-.26, p<.01)$

\section{Role of DT in Acquired Capability for Suicide}

In a cross-sectional study among undergraduate students $(N=283,59 \%$ female, 69\% White, mean age $=19$ ), Anestis, Bagge, et al. (2011) found that self-reported DT (as 
measured by the sDTS) was positively correlated with the acquired capability for suicide $(r=.17, p<.01)$. Yet, DT did not predict acquired capability for suicide $(t=.62, \mathrm{p}=.53)$ after controlling for painful and/or provocative events, sensation seeking, positive urgency (acting impulsively in response to positive emotions), negative urgency (acting impulsively in response to negative emotions), lack of premeditation (acting without planning), lack of perseverance (quitting when tasks become difficult), depression, anxiety, and sex.

In the same sample, Anestis and Joiner (2012) found that behaviorally-assessed DT (as measured by the DTT) was also positively correlated with the acquired capability for suicide $(r=.18, p<.01)$. In contrast to self-reported DT, the behaviorally-assessed DT did predict the acquired capability for suicide such that higher levels of DT were associated with higher acquired capability for suicide, after accounting for sex and number of painful/provocative events $(\beta=.17, t=3.01, p=.00)$. DT also interacted with painful/provocative experiences in the prediction of acquired capability for suicide, such that the predictive power of painful/provocative events is greater at higher levels of DT $(\beta$ $=.23, t=3.36, p=.00)$.

In a cross-sectional study among a different sample of undergraduate students $(N$ $=200,69 \%$ female, $68 \%$ Caucasian, mean age $=18)$, Anestis, Bender, et al. $(2011)$ found that self-reported DT (as measured by the sDTS) was positively correlated with acquired capability for suicide only in males $(r=.56, p<.001)$. They further found that both DT $(\beta=0.2, t=3.24, p=.001)$ and the interaction of DT and gender $(\beta=-0.7, t=-2.35, p=$ .02) predicted the acquired capability for suicide, such that men with high DT had the highest acquired capability for suicide, after accounting for sensation seeking. In the 
same sample, Bender et al. (2012) found that self-reported DT interacted with sensation seeking to predict the acquired capability for suicide, after controlling for sex, negative urgency, lack of premeditation, lack of perseverance, depression, anxiety, AS, and perfectionism $(t=2.373, p=.019)$.

In a third sample of undergraduate students $(N=220,77 \%$ female, $59 \%$ Caucasian, mean age $=21$, Anestis, Moberg, et al. (2014) again found self-reported DT (as measured by the sDTS) to be positively correlated with acquired capability for suicide $(r=.25, p<.01)$. As noted above, they also found that DT fully mediated the predictive relationship between trait hope and acquired capability for suicide after controlling for sex, age, income, and painful and/or provocative events. The difference between the results of Anestis, Bagge, et al. (2011) and the results of the other authors may be accounted for by the difference in covariates considered.

\section{DT, NSSI, and Suicide Considered Together}

In a sample of inpatients receiving treatment for SUDs $(N=93,55 \%$ male, $76-$ 78\% Caucasian, mean age $=36$ ), Anestis, Knorr, et al. (2013) and Anestis, Pennings, et al. (2013) examined the interaction of DT and NSSI in the prediction of suicide. Distress tolerance was assessed through self-report (the sDTS). The participants also completed a self-report of the lifetime number of suicide attempts (used as the outcome measure by Anestis, Pennings, et al., 2013) and an interview from which the highest lethality of the participant's most lethal suicide attempt was drawn (used as the outcome measure by Anestis, Knorr, et al., 2013).

Anestis, Knorr, et al. (2013) found that DT interacted with NSSI lifetime frequency (but not the number of NSSI methods) in the prediction of suicide potential, 
after controlling for sex, age, income, marital status, and depression $(\beta=.21, p<.05)$. As such, the magnitude of the relationship between NSSI frequency and suicide potential was greatest at high levels of DT. Of note, the interaction of DT with NSSI methods approached significance and trended toward the same pattern $(\beta=.18, p=.053)$. These results indicated that greater lifetime frequency of NSSI was associated with suicide potential, particularly for individuals with high DT levels.

Anestis, Pennings, et al. (2013) found that the predictive relationship between DT and lifetime suicide attempts was mediated by NSSI lifetime frequency, after controlling for sex, income, age, and depression (Bootstrap coefficient $=-.0034,95 \% \mathrm{CI}$ $[-.0080,-.0003])$. The authors suggested that, "It is not low distress tolerance per se but certain types of behaviors used to escape or avoid unwanted emotional distress that explains the elevated rates of suicidal behavior among populations with low distress tolerance" (p. 999).

An advantage of these two studies was the use of an inpatient sample which allowed for a high prevalence of past suicide attempt and lifetime NSSI frequency. The studies also controlled for sex, age, income, and depression. Shortcomings of the studies included a largely Caucasian sample and a relatively small sample size.

Of note, Anestis, Kleiman, et al. (2014) similarly found that the predictive relationship between DT and lifetime suicide attempts was partially mediated by NSSI lifetime frequency in a large undergraduate sample $(N=1317)$. Overall, these three studies demonstrate that DT and NSSI lifetime frequency interact in the prediction of lifetime suicide attempts in both inpatient and undergraduate samples. 


\section{DT and Self-Damaging Behaviors Summary}

In summary, the existing literature supports a relationship between DT and selfdamaging behaviors. Distress tolerance is negatively correlated with unhealthy eating behaviors (e.g., body disordered eating patterns, bulimic symptoms, body dissatisfaction, disinhibited eating, desire to be thinner, and binge eating symptoms). For some symptoms, the relationship between DT and eating pathology is specifically related to an aspect of DT involving behavioral avoidance of positive affect. Distress tolerance is also negatively correlated with both NSSI lifetime frequency and number of NSSI methods. An intervention aimed at increasing DT skills successfully decreased NSSI frequency. Additionally, self-reported DT has been shown to be negatively correlated with suicidal desire, suicide potential, and lifetime suicide attempts. This pattern contrasts with a positive correlation observed between self-reported DT and acquired capability for suicide. Overall, there is strong evidence for the role of DT in these severe, selfdamaging behaviors. So, a more comprehensive model for individual difference factors in DT is needed in order to better inform the development of prevention and intervention protocols to reduce these risks.

\section{Summary}

In conclusion, emotional DT is defined as one's perceived ability to withstand negative emotional states. It is quite stable across time, unless targeted attempts to change DT level are made through clinical intervention. Distress tolerance is a concept with importance across multiple diagnostic categories. For instance, the existing literature strongly supports a relationship between DT and EDs, NSSI, and suicidality. These behaviors are important for study because they cause physical harm to the individuals 
engaging in them and also because individuals engaging in these behaviors are frequent consumers of clinical services.

Despite the clinical importance of DT, little is known about factors accounting for individual differences in level of DT. This lack of knowledge hinders the development of preventative programs and clinical interventions. Drawing from Linehan's biosocial theory of emotional dysregulation and Lynch and Mizon's model of individual difference factors in DT, I proposed that emotional reactivity and learned helplessness are individual difference factors in DT. Indeed, research supports the likelihood of emotional reactivity being an individual difference factor in DT and appears to relate to a wide variety of negative behaviors in a similar manner as DT. Only a few studies suggest that DT and emotional reactivity relate to negative behaviors differently. Though no literature speaks directly to the relationship between learned helplessness and DT, the relationship is theoretically supported and has not been empirically refuted.

Thus, in this study, I tested two models (a two-week model and a lifetime model) of individual difference factors (emotional reactivity and learned helplessness) in emotional DT in the context of self-damaging behaviors (restricting, fasting, binging, purging, NSSI, suicidal ideation, and suicide attempts). In the literature reviewed above, the statitical procedures utilized were relatively basic. Though the studies may have used more advanced statistics for their main hypotheses, the statical procedures used to examine DT in relation to self-harming behaviors were often limited to correlation and regression analyses. To remedy this weakness, I utilized SEM in my study. Structural equation modeling has many advantages including accounting for error in the independent variables and testing multivariate hypotheses simultaneously. My research 
design and statistical approach will be explained more thoroughly in the following chapter. 


\section{CHAPTER 3}

\section{METHODOLOGY}

\section{Introduction}

In this chapter, I will describe the research design that I used to examine individual difference factors related to DT and self-damaging behaviors. This study used survey research as part of a non-experimental research design. Subjects completed a demographic questionnaire and surveys measuring their levels of (a) emotional reactivity, (b) learned helplessness, (c) DT, (d) depression, and (e) anxiety, (f) two-week frequency of self-damaging behaviors, and (g) lifetime frequency of self-damaging behaviors. Structural equation modeling was used to test two proposed models of the relationship between participants' emotional reactivity, learned helplessness, DT, and frequency of self-damaging behaviors (one model for two-week frequency of self-damaging behaviors and another model for lifetime frequency).

\section{Research Design}

This study employed a nonexperimental research design. A nonexperimental design was appropriate for my study since I desired to measure the variables as they are experienced in the respondents' daily contexts, rather than in an experimental environment. Also, experimental manipulation of the variables of interest would have been unethical and dangerous given the nature of self-damaging behaviors. However, a 
limitation of nonexperimental research design is that the results may speak to correlation and prediction but not causation (since many variables cannot be controlled for in a nonexperimental setting).

My data was gathered using survey research. Survey research was appropriate for my study since my variables of interest are subjective, focused on the respondents' perceived levels of emotional reactivity, learned helplessness, distress tolerance, anxiety, and depression as well as their perceived (or recalled) frequency of self-damaging behaviors. Thus, these variables must be assessed through self-report. A limitation of self-report measures is that respondents may have answered surveys in ways which were socially desirable, rather than in ways which accurately reflected their true behavior. However, my study was intended to be applicable in clinical work in which a clinician's data is primarily client self-report through narrative or brief assessments. Therefore, assessing participants' self-perceived level of the variables of interest was appropriate for my study's intent.

Another data collection method sometimes used for self-report data is personal interviews. However, survey research is more easily administered and is administered at lower cost in comparison to in-person interviews. Additionally, surveys produce immediate quantitative data, whereas interview responses must be coded into quantitative data (which often introduces researcher bias and rater error). Survey research can also be conducted online, which allows for a sample from a broader geographic area than inperson interviews would dictate. Further, given the sensitive nature of the variables of interest in this study, anonymous surveys may have allowed for more open sharing of 
risky behaviors than would have been likely with in-person interviews with a stranger (Palmgreen et al., 2002, as cited in Beck et al., 2013).

A limitation of the survey approach was the potential to collect less expansive or nuanced data than would have been elicited in an interview format. Also, since a survey approach did not involve a controlled experimental environment, it is unknown whether immediate contextual factors (e.g., loud noise, cold room temperature, etc.) might have influenced respondents' answers. Further, since my survey was conducted online it limited my sample to individuals who have internet access and are comfortable with the use of the internet. Additionally, it may be that individuals who voluntarily sign up to complete online surveys differ from the general population in their level of the variables of interest.

\section{Population and Sample}

The sample for this study consisted of adults, 18 years or older, who resided in the United States of America. Screening questions ensured that the participants were 18 years or older and that the participants had experienced at least one of the self-damaging behaviors of interest. The sample was collected using convenience sampling. The sample was drawn from respondents who were signed up to complete surveys on QuestionPro, which is an online platform which helps researchers distribute surveys to target populations. QuestionPro allows participants to receive points in exchange for completed surveys. These accumulated points can be redeemed for $\$ 10.00$ gift certificates to popular stores and restaurants. QuestionPro was selected as the platform for my study because it allowed for long surveys with ease, protected the anonymity of participants, had historically collected data rapidly for other students in our department, and offered me a 
discount for being a doctoral student researcher. A sample size of 300 was desired in order to allow for adequate statistical power in the SEM. This quantity was calculated based on the number of observed and latent variables in the longest survey being used (the ERS).

QuestionPro utilizes a Survey Analytics panel of over 5 million people globally who have voluntarily signed up to complete research surveys (Survey Analytics, 2010). As of 2010, 2.3 million of these members are from within the United States. Members designate how many survey invitations they would like to receive per month. In order to reduce respondent fatigue, participants are allowed to receive no more than 8 invitations per month. Survey Analytics utilizes a double opt-in procedure which requires both a rigorous registration process (over 300 data points collected) and periodic profile updates. Survey Analytics has a low attrition rate, with only $2 \%$ of their members opting to unsubscribe from the panel per year.

The gender division of the Survey Analytics panel closely aligns ( male $=46 \%$ ) with the gender division of the general online population (male $=47 \%$; statistics for panel members and the general online population drawn from Survey Analytics, 2010) and the general USA population ( male $=49 \%$; statistics for the general USA population drawn from United States Census Bureau, 2014). The age representation of members (18-24: $22 \%, 25-34: 31 \%, 35-44: 16 \%, 45-54: 28 \%, 55+: 8 \%$ ) aligns more loosely with the age representation of the general online population (18-24: 13\%, 25-34: $20 \%$, 35-44: $24 \%$, 45-54: $24 \%, 55+: 20 \%$ ), as there is greater representation of younger individuals in the Survey Analytics panel than in the general online population. The age representation of members differs from the age representation of the general USA population, but this is to 
be expected since individuals under the age of 18 compose $23 \%$ of the general USA population. Additionally, representation of minority ethnicities among panel members (White $/$ Caucasian $=80 \%$, African American $=8 \%$, Hispanic $=7 \%$, American Indian=6\%, Asian $=5 \%$ ) matches or exceeds representation of minority ethnicities in the general online population (White/Caucasian=87\%, African American=7\%, Hispanic $=5 \%$, American Indian=4\%, Asian=6\%). However, representation of Caucasians among members is slightly greater than representation of Caucasians in the general USA population (White/Caucasian=77\%). While QuestionPro allows for targeting representation of these demographic divisions, the demographics of my sample were filtered by the initial question about experience with self-damaging behaviors.

Use of online platforms for survey distribution is a common and growing approach among researchers. For example, in a survey of 750 university human research ethics boards, Buchanan and Hvizdak (2009) found that $94 \%$ of respondents indicated that studies involving the use of an online platform are their most frequently reviewed type of study. Specifically, QuestionPro has been used as a survey hosting platform for research published in a variety of journals including the Australian \& New Zealand Journal of Psychiatry (e.g., Porter, Starcevic, Berle, \& Fenech, 2010), Autism (e.g., G. Mitchell \& Locke, 2015), Current Research in Social Psychology (e.g., Sayama \& Sayama, 2011), The Family Journal (e.g., Hertlein, Blumer, \& Mihaloliakos, 2015), and Remedial and Special Education (e.g., Cawthon, 2011). Additionally, a study by Braun and Turner (2014) was published in the Consulting Psychology Journal: Practice and Research which utilized the QuestionPro panel for sample recruitment. There are likely other studies published which have used the QuestionPro panel for sample recruitment, 
but they are difficult to search for given that this information is not typically included in an article's abstract. A similar online platform, Amazon's Mechanical Turk, is a similar large online sample pool (see Mason \& Suri, 2012). Research utilizing Mechanical Turk has been widely published, as evidenced by its use in two studies included in this literature review: Bardeen et al. (2013) and Fergus et al. (2015). A brief search of Sage Journal's publications revealed over 1700 journal articles including the term "Mechanical Turk" (though some of these results are articles about the use of Mechanical Turk). Given the similarities between the sample pools of Mechanical Turk and QuestionPro, it was anticipated that publications will be open to the use of QuestionPro.

\section{Hypotheses and Research Questions}

The first hypothesis of this study was that the reproduced covariance matrices $\Sigma(\gamma)$ implied in the two-week frequency theoretical model (Figure 1) and the observed sample covariance matrices $S$ were equal. This hypothesis addressed the following research question: is the hypothesized two-week frequency model a good fit to the sample? As depicted, the two-week frequency model proposed a direct effect between emotional reactivity and DT, between learned helplessness and DT, and between DT and two-week frequency of self-damaging behaviors. It also proposed an indirect effect between emotional reactivity and two-week frequency of self-damaging behaviors and between learned helplessness and two-week frequency of self-damaging behaviors. 


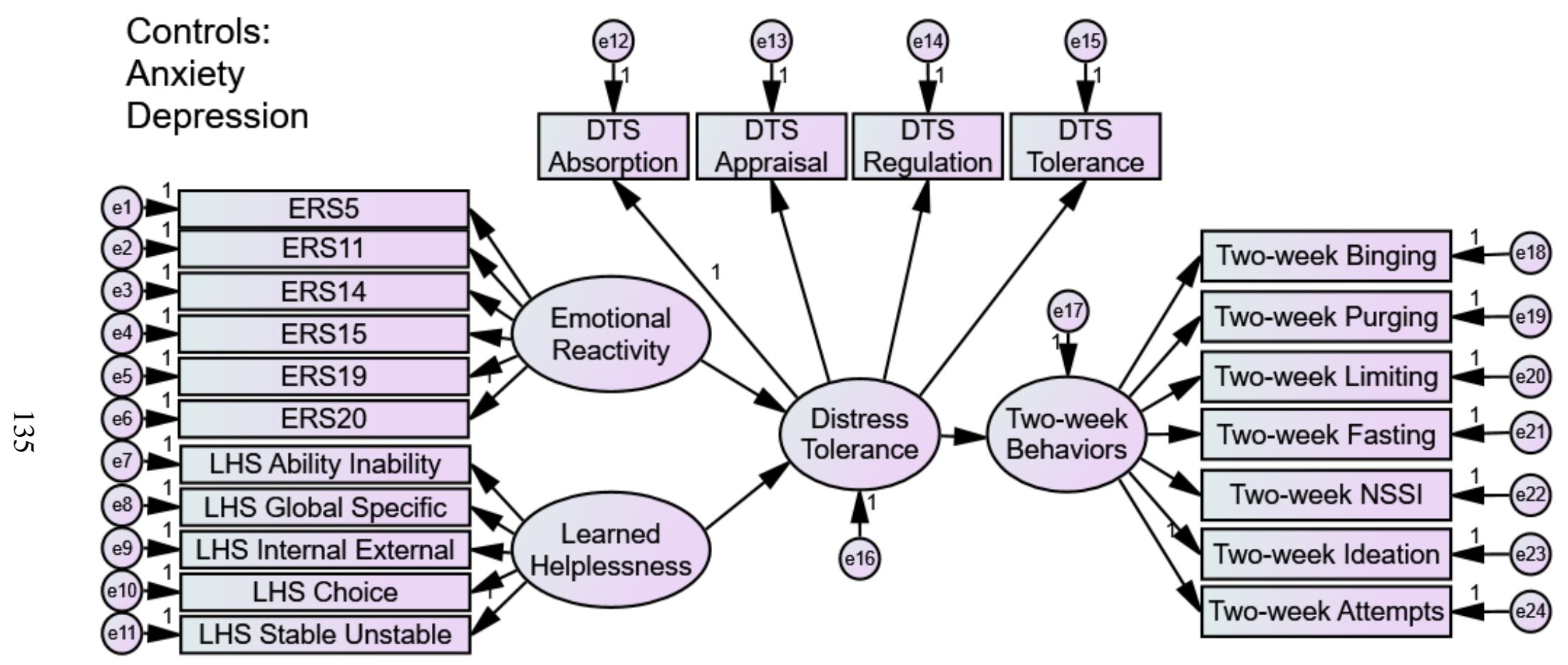

Figure 1: Hypothesized Two-Week Frequency Model 
The second hypothesis of this study was that the reproduced covariance matrices $\Sigma(\gamma)$ implied in the lifetime frequency theoretical model (Figure 2) and the observed sample covariance matrices $S$ were equal. This hypothesis addressed the following research question: is the hypothesized lifetime frequency model a good fit to the sample? As depicted, the lifetime frequency model proposed a direct effect between emotional reactivity and DT, between learned helplessness and DT, and between DT and lifetime frequency of self-damaging behaviors. It also proposed an indirect effect between emotional reactivity and lifetime frequency of self-damaging behaviors and between learned helplessness and lifetime frequency of self-damaging behaviors.

\section{Variables Definition}

\section{Exogenous Variables}

This study examined the following exogenous (independent) variables: age, anxiety, depression, emotional reactivity, and learned helplessness. This section and the next section will describe the conceptual definitions of each variable and the instruments which were used to measure each variable. The psychometric properties of the scales and specifics of the items used are described in the Instrumentation section.

Age was measured from a free-form response on the demographic information questionnaire. Age was included as a control in the lifetime frequency model because individuals who have lived longer have the potential to have engaged in self-damaging behaviors more times over a longer lifetime than individuals who are younger (e.g., an individual who started purging once a week at age 20 and is now age 30 will have a higher lifetime frequency of purging than an individual who also started purging once a week at age 20 but is currently only 22 years old). 


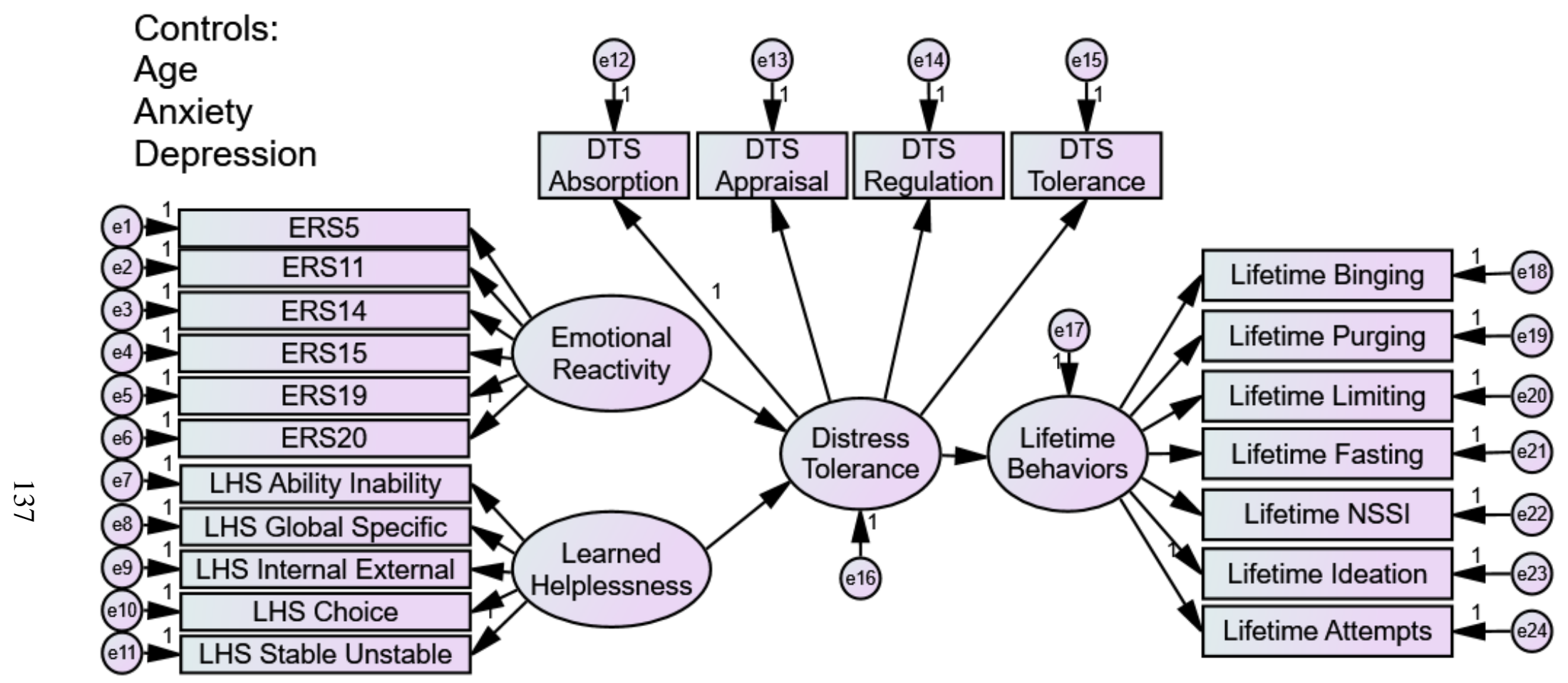

Figure 2. Hypothesized Lifetime Frequency Model 
Anxiety was defined as the experience of the following symptoms: dry mouth, rapid breathing in the absence of physical exertion, trembling, panic, worry about panic, feeling scared without any good reason, and changes in heart action in the absence of physical exertion (Lovibond \& Lovibond, 1995). This variable was measured using the Anxiety subscale of the 21-item version of the Depression Anxiety Stress Scales 21 (DASS-21). Given the documented relationship between anxiety and self-damaging behaviors, anxiety was used as a control in both the lifetime frequency and two-week frequency models.

Depression was defined as the experience of the following symptoms: lack of positive feelings, lack of initiative, lack of anything to look forward to, lack of enthusiasm, feeling downhearted, feeling worthless, and feeling that life is meaningless (Lovibond \& Lovibond, 1995). This variable was measured using the Depression subscale of DASS-21. Given the documented relationship between depression and selfdamaging behaviors, depression was used as a control in both the lifetime frequency and two-week frequency models.

Emotional reactivity was defined as the extent to which an individual experiences the intensity, sensitivity, and persistence of emotions (Nock et al., 2008). This variable was measured using the ERS (Nock et al., 2008).

Learned helplessness was defined as the extent to which an individual believes that outcomes are independent of his/her behavior (Eisenberger et al., 1976). This variable was measured using the LHS (Quinless \& Nelson, 1988). 


\section{Endogenous Variables}

The present study examined the following endogenous (dependent) variables: distress tolerance, lifetime frequency of self-damaging behaviors, and two-week frequency of self-damaging behaviors.

DT was defined as one's perceived ability to withstand negative emotional states (Simons \& Gaher, 2005; Zvolensky et al., 2011). This variable was measured using the sDTS (Simons \& Gaher, 2005). Distress tolerance was represented by a latent variable composed of scores for each of the four subscales of the sDTS.

Two-week frequency of self-damaging behaviors was defined as the two-week incidence of binge eating, purging, restricted (limiting) eating, restricted (fasting) eating, NSSI, suicidal ideation, and suicide attempts. Two-week frequency of self-damaging behaviors was represented by a latent variable composed of items measuring two-week frequency of binge eating, purging, limiting, fasting, NSSI, suicidal ideation, and suicide attempts.

Lifetime frequency of self-damaging behaviors was defined as the lifetime incidence of binge eating, purging, restricted (limiting) eating, restricted (fasting) eating, NSSI, suicidal ideation, and suicide attempts. Lifetime frequency of self-damaging behaviors was represented by a latent variable composed of items measuring lifetime frequency of binge eating, purging, limiting, fasting, NSSI, suicidal ideation, and suicide attempts.

Table 1 lists the conceptual definitions of the variables included in my study. It also depicts the survey items used to measure each variable. For variables drawn from 
instruments with multiple subscales, this will give the reader a general idea of what each subscale represents.

\section{Instrumentation}

\section{Depression Anxiety Stress Scales}

The Depression Anxiety Stress Scales, 21 item version (DASS-21; Lovibond \& Lovibond, 1995) was adapted from the Depression Anxiety Stress Scales, 42 items version. The DASS-21 consists of 3 subscales (depression, anxiety, and stress) with 7 items each. For this study, only the depression and anxiety subscales were used. Respondents rated the extent to which each of the 21 items was applicable to them over the past week on a Likert scale from 0 (did not apply to me at all) to 3 (applied to me very much, or most of the time). Example items for the depression subscale are "I felt that life was meaningless" and "I felt that I had nothing to look forward to." Example items for the anxiety subscale are "I was aware of the action of my heart in the absence of physical exertion (e.g., sense of heart rate increase, heart missing a beat)" and "I experienced difficulty breathing (e.g., excessively rapid breathing, breathlessness in the absence of physical exertion)." Higher scores represent more depression or anxiety.

Antony, Bieling, Cox, Enns, and Swinson (1998) conducted factor analysis on the DASS-21 with outpatients with panic disorder $(N=67,64 \%$ female, mean age $=37)$, outpatients with obsessive-compulsive disorder $(N=54,57 \%$ male, mean age $=36)$, outpatients with social phobia $(N=74,66 \%$ male, mean age $=35)$, outpatients with specific phobia $(N=17,78 \%$ female, mean age $=34)$, outpatients with $\operatorname{MDD}(N=46$, $64 \%$ male, mean age $=45)$, and nonclinical volunteers $(N=49,61 \%$ female, mean age $=$ 28). Results of EFA among the clinical sample $(N=258)$ revealed 3 factors (factor 
Table 1

Conceptual Definitions of Variables and Source of Associated Survey Items

\begin{tabular}{|c|c|c|}
\hline $\begin{array}{l}\text { Variable name } \\
\& \text { Instrument(s) }\end{array}$ & Conceptual definition & $\begin{array}{l}\text { Instrument, subscale ,\& } \\
\text { items used }\end{array}$ \\
\hline $\begin{array}{l}\text { Anxiety } \\
\text { (measured by } \\
\text { items from the } \\
\text { DASS-21) }\end{array}$ & $\begin{array}{l}\text { The experience of the following } \\
\text { symptoms: dry mouth, rapid breathing } \\
\text { in the absence of physical exertion, } \\
\text { trembling, panic, worry about panic, } \\
\text { feeling scared without any good } \\
\text { reason, and changes in heart action in } \\
\text { the absence of physical exertion } \\
\text { (Lovibond \& Lovibond, 1995) }\end{array}$ & $\begin{array}{l}\text { DASS-21: } \\
\text { Anxiety }(2,4,7,9,15,19 \text {, } \\
\text { 20) }\end{array}$ \\
\hline $\begin{array}{l}\text { Depression } \\
\text { (measured by } \\
\text { items from the } \\
\text { DASS-21) }\end{array}$ & $\begin{array}{l}\text { The experience of the following } \\
\text { symptoms: lack of positive feelings, } \\
\text { lack of initiative, lack of anything to } \\
\text { look forward to, lack of enthusiasm, } \\
\text { feeling downhearted, feeling worthless, } \\
\text { and feeling that life is meaningless } \\
\text { (Lovibond \& Lovibond, 1995) }\end{array}$ & $\begin{array}{l}\text { DASS-21: } \\
\text { Depression }(3,5,10,13, \\
16,17,21)\end{array}$ \\
\hline $\begin{array}{l}\text { Emotional } \\
\text { reactivity } \\
\text { (measured by } \\
\text { the ERS) }\end{array}$ & $\begin{array}{l}\text { The extent to which an individual } \\
\text { experiences the intensity, sensitivity, } \\
\text { and persistence of emotions (Nock et } \\
\text { al., 2008) }\end{array}$ & All ERS items, no subscales \\
\hline $\begin{array}{l}\text { Distress } \\
\text { tolerance } \\
\text { (measured by } \\
\text { the sDTS) }\end{array}$ & $\begin{array}{l}\text { One's perceived ability to withstand } \\
\text { negative emotional states (Simons \& } \\
\text { Gaher, 2005; Zvolensky et al., 2011) }\end{array}$ & $\begin{array}{l}\text { sDTS: } \\
\text { Absorption }(2,4,15) \\
\text { Appraisal }(6,7,9,10,11, \\
12) \\
\text { Regulation }(8,13,14) \\
\text { Tolerance }(1,3,5)\end{array}$ \\
\hline $\begin{array}{l}\text { Learned } \\
\text { helplessness } \\
\text { (measured by } \\
\text { the LHS) }\end{array}$ & $\begin{array}{l}\text { The extent to which an individual } \\
\text { believes that outcomes are independent } \\
\text { of his/her behavior (Eisenberger et al., } \\
\text { 1976) }\end{array}$ & $\begin{array}{l}\text { LHS: } \\
\text { Ability-Inability }(2,17) \\
\text { Choice }(4,14) \\
\text { Global-Specific }(9,16,18, \\
19,20) \\
\text { Internal-External }(7,8,10, \\
11,12) \\
\text { Stable-Unstable }(1,3,5,6 \text {, } \\
13,15)\end{array}$ \\
\hline
\end{tabular}


Table 1-Continued

\begin{tabular}{|c|c|c|}
\hline $\begin{array}{l}\text { Variable name } \\
\& \text { Instrument(s) }\end{array}$ & Conceptual definition & $\begin{array}{l}\text { Instrument, subscale }, \& \\
\text { items used }\end{array}$ \\
\hline $\begin{array}{l}\text { Two-week } \\
\text { frequency of } \\
\text { self-damaging } \\
\text { behaviors } \\
\text { (Measured by } \\
\text { items from the } \\
\text { EDE-Q and } \\
\text { SITBI) }\end{array}$ & $\begin{array}{l}\text { The two-week incidence of binge } \\
\text { eating, purging, limiting, fasting, } \\
\text { NSSI, suicidal ideation, and suicide } \\
\text { attempts }\end{array}$ & $\begin{array}{l}\text { EDE-Q: } \\
\text { Restraint }(1,2) \\
\text { Frequency }(15,16) \\
\text { SITBI: } \\
\text { Suicidal ideation }(7) \\
\text { Suicide attempts }(91) \\
\text { NSSI }(149)\end{array}$ \\
\hline $\begin{array}{l}\text { Lifetime } \\
\text { frequency of } \\
\text { self-damaging } \\
\text { behaviors } \\
\text { (Measured by } \\
\text { items from the } \\
\text { EDE-Q and } \\
\text { SITBI) }\end{array}$ & $\begin{array}{l}\text { The lifetime incidence of binge eating, } \\
\text { purging, limiting, fasting, NSSI, } \\
\text { suicidal ideation, and suicide attempts }\end{array}$ & $\begin{array}{l}\text { EDE-Q: } \\
\text { Restraint }(1,2) \\
\text { Frequency }(13,14,16) \\
\text { SITBI: } \\
\text { Suicidal ideation }(4) \\
\text { Suicide attempts }(84,88) \\
\text { NSSI }(116,146)\end{array}$ \\
\hline
\end{tabular}

loadings ranging from .48 to .84 ), which together accounted for $67 \%$ of the variance in scores.

In the full sample, the subscales demonstrated good internal consistency (Depression: $\alpha=.94$; Anxiety: $\alpha=.87$ ). Convergent validity was demonstrated in that the depression subscale correlated strongly $(r=.79)$ with the Beck Depression Inventory (Beck, Rush, Shaw, \& Emery,1979, as cited in Antony et al., 1998, p. 177) and the anxiety subscale correlated strongly $(r=.85)$ with the Beck Anxiety Inventory (Beck \& Steer, 1990, as cited in Antony et al., 1998, p. 177). Criterion validity was demonstrated in that, among the clinical groups, the group with MDD was the highest scoring group on the depression subscale and the group with panic disorder was the highest scoring group on the anxiety subscale. Additionally, individuals in the comparison group scored lower 
on both subscales than all of the clinical groups (however, the difference in mean age between the clinical groups and the comparison group was not controlled for in this calculation).

\section{Distress Tolerance Scale}

The sDTS (Simons \& Gaher, 2005) consists of 15 items that assess DT on a Likert scale. Respondents rated their agreement with each of the 15 items from 1 (strongly agree) to 5 (strongly disagree). One item was reverse scored. The sDTS is composed of four subscales: absorption, appraisal, regulation, and tolerance. These subscales and the factor analysis supporting them were described more thoroughly in the previous chapter. The factor analysis revealed one higher-order factor (total DT, calculated as the mean of the four lower-order factors) and four lower-order factors (the four subscales). First, absorption represents an individual reporting that his/her attention is consumed "by the presence of distressing emotions and that [his/her] functioning is significantly disrupted by the experience of negative emotions" (p. 84). An example item for this subscale is "When I feel distressed or upset, I cannot help but concentrate on how bad the distress actually feels" (p. 93). Second, appraisal represents an individual's "lack

of acceptance of distress, being ashamed of being distressed, and perceiving one's coping abilities as inferior to others" (p. 84). An example item for this subscale is "My feelings of distress or being upset scare me" (p. 93). Third, regulation represents an individual making "great efforts to avoid negative emotions and utilizing rapid means of alleviating the negative emotions" he/she experiences (p. 84). An example item for this subscale is "I'll do anything to stop feeling distressed or upset" (p. 93). Fourth, tolerance represents an individual "report[ing] distress as being unbearable" and being unable to "handle 
being distressed or upset" (Simons \& Gaher, 2005, p. 84). An example item for this subscale is "I can't handle feeling distressed or upset" (p. 93). On each subscale, a higher score represents higher DT.

Simons and Gaher (2005) examined the validity of the sDTS among a sample of undergraduate students $(N=642,70 \%$ female, $89 \%$ Caucasian, mean age $=20)$. Discriminant validity was demonstrated in that DT was correlated with, but yet distinct from affect dysregulation (26\% shared variance), affective distress (35\% shared variance), and negative mood regulation expectancies (29\% shared variance). Convergent validity was demonstrated in that DT was more strongly associated with mood acceptance (not needing to change the mood, $r=.47$ ) than mood typicality (the frequency of the mood, $r=.17)$. Criterion validity was demonstrated in that DT was negatively correlated with alcohol coping motives $(r=-.23)$ and marijuana coping motives $(r$ $=-.20)$.

In a second sample of undergraduate students $(N=823,67 \%$ female, $94 \%$ Caucasian, mean age $=20$ ), Simons and Gaher (2005) examined the reliability, criterion validity, and internal consistency of the sDTS. Participants completed the sDTS at baseline and then at an average of 216 days later. Results indicated that DT was negatively correlated with alcohol use related problems $(r=-.23)$. Results also indicated moderate test-retest reliability $(\mathrm{ICC}=.61)$. Macatee et al. $(2015)$ found identical testretest reliability $(\mathrm{ICC}=.61)$ for the sDTS across a one-month interval among undergraduate students $(N=87,76 \%$ female, $67 \%$ Caucasian, mean age $=19)$. Simons and Gaher (2005) found good internal consistency for the higher-order factor (Time 1: $\alpha$ $=.82$, Time 2: $\alpha=.85$ ). Internal consistency for the lower-order factors was adequate, but 
lower than that for the higher-order factors (Time 1: $\alpha=.70$ to .82 , Time $2: \alpha=.73$ to $.84)$.

\section{Emotion Reactivity Scale}

The ERS (Nock et al., 2008) consists of 21 items that assess emotional reactivity on a Likert scale. Respondents rated their agreement with each of the 21 items from 0 (not at all like me) to 4 (completely like me). Though the ERS has three subscales (Arousal/Intensity, Persistence, and Sensitivity) which have strong internal consistency, a single-factor has proven a better fit for the instrument. The factor analysis supporting this conclusion was described more thoroughly in the previous chapter. So, the scale was utilized as a unidimensional measure. Example items include: "I tend to get very emotional very quickly," "Even the littlest things make me emotional," and "When I experience emotions, I feel them very strongly/intensely" (p. 111). A higher score represents more emotional reactivity.

In a sample of outpatients ranging from 12 to 19 years old $(N=94,78 \%$ female, 72\% Caucasian, Mean age $=17$ ), Nock et al. (2008) found that the total score demonstrated good internal consistency $(\alpha=.94)$. The total score also demonstrated strong internal consistency $(\alpha=.92)$ in a study conducted by Franklin et al. (2014) with a nonclinical sample of adults with a history of NSSI $(N=49,73 \%$ female, $61 \%$ Caucasian, mean age $=24)$. In the study by Nock et al. (2008), construct validity was demonstrated in that the ERS had positive correlations with reactivity to negative events (as measured by the Behavioral Inhibition Scale, Carver \& White, 1994, as cited on p. $109 ; r=.37, p<.01)$, depressive $\operatorname{mood}(r=.61, p<.01)$, and frustration $(r=.53, p<$ $.01)$, as well as negative correlations with attention $(r=-.45, p<.05)$ and inhibitory 
control $(r=-.45, p<.05)$. The ERS also had nonsignificant correlations with affiliation $(r$ $=-.02, n s)$, drive $(r=-.09, n s)$, fun seeking $(r=-.20, n s)$, high intensity pleasure $(r=-.07$, $n s)$, pleasure sensitivity $(r=.13, n s)$, and reward responsiveness $(r=-.16, n s)$. As reported more fully in the previous chapter, criterion validity was demonstrated in that individuals with an anxiety disorder reported more emotional reactivity than individuals without an anxiety disorder; individuals with an ED reported more emotional reactivity than individuals without an ED; and individuals with mood disorders reported more emotional reactivity than individuals without a mood disorder. However, individuals with a SUD did not report more emotional reactivity than individuals without a SUD and individuals with a disruptive behavior disorder did not report more emotional reactivity than individuals without a disruptive behavior disorder.

\section{Learned Helplessness Scale}

The LHS Form B (Quinless \& Nelson, 1988) consists of 20 items that assess learned helplessness on a Likert Scale. Respondents rated the degree to which each of the 20 items describe them from 1 (strongly disagree) to 4 (strongly agree). Half of the items are reverse scored. Factor analysis in a sample of healthy adults $(N=241,72 \%$ female, Mean age $=40$ ) yielded five factors: Internality-Externality, Globality-Specificity, Stable-Unstable, Ability-Inability to Control, and Individual's Choice of Situations in Which the Person Intentionally Participates (called Choice in this narrative for clarity). Together, the factors accounted for $47 \%$ of the variance in scores. Of note, AbilityInability to Control and Choice each contain only two items, but were retained based on theory. Example items include "I try new tasks if I have failed similar ones in the past" (Reverse scored), "No matter how hard I try, things never seem to work out the way I 
want them to", and "No matter how much energy I put into a task, I feel I have no control over the outcome." Higher scores indicate more learned helplessness.

Internal consistency was demonstrated for the LHS in the healthy control sample $(\alpha=.85)$. Internal consistency was also demonstrated in a sample of male oncology patients $(N=24$, mean age $=60 ; \alpha=.83)$, a sample of male hemodialysis patients $(N=$ 30 , mean age $=61 ; \alpha=.92)$, and a sample of patients with spinal cord injury $(N=20$, mean age $=48 ; \alpha=.94)$. Construct validity was demonstrated in that the LHS was positively correlated with hopelessness $(r=.25)$ and negatively correlated with selfesteem $(r=-.62)$.

In a sample of male Vietnam veterans $(\mathrm{N}=88,81 \%$ Caucasian, mean age $=53)$, McKeever, McWhirter, and Huff (2006) found that the LHS was positively correlated with posttraumatic stress disorder symptom severity $(r \mathrm{~s}=.22, p<.05)$. However, this correlation was no longer significant once a Bonferroni correction for Type 1 error was made. In a sample of male adults who were sexually abused as children $(N=49$, mean age of participants abused by a priest $=51$, mean age of participants abused by a lay person $=42$ ), Shea (2008) found that the LHS was positively correlated with depression severity $(r=.48, p=.00)$.

\section{Self-Damaging Behaviors Questionnaire}

In order to assess the two-week frequency of self-damaging behaviors and lifetime frequency of self-damaging behaviors, I combined items from two different scales. First, select items from the Self-Injurious Thoughts and Behaviors Interview Long form (SITBI; Nock et al., 2007) were used to assess the frequency of suicidal ideation, suicide attempts, and NSSI. Some items were adapted to reflect two-week, 
rather than one-week frequency. The SITBI consists of 169 items which vary in response format (multiple-choice, Likert scale, dichotomous, quantitative, and open-ended). The items I utilized all ask for a quantitative, free-response reflecting frequency of the behavior being queried. Higher numbers indicate higher frequencies of the behaviors.

\section{SITBI}

The SITBI was initially validated with an adolescent sample $(N=94,78 \%$ female, mean age $=17$ ). The SITBI is designed to assess six different constructs: suicidal ideation, suicide plans, suicide gestures, suicide attempts, thoughts of NSSI, and NSSI. As such, factor analyses and internal consistency analyses were not run since the SITBI assesses more than one construct. Test-retest reliability was assessed at baseline and 6 months later. When comparing the reliability of reporting the presence or absence of lifetime behaviors, test-retest reliability was strong for suicidal ideation $(\kappa=0.70)$, suicide plan $(\kappa=0.71)$, suicide attempt $(\kappa=0.80)$, and NSSI $(\kappa=1.0)$. However, testretest reliability was poor for the lifetime presence or absence of suicidal gestures $(\kappa=$ 0.25). Test-retest reliability for the reported frequency of behaviors was strong for suicidal ideation $(\mathrm{ICC}=.74, p<.001)$, suicide attempts $(\mathrm{ICC}=.50, p<.001)$, and NSSI $(\mathrm{ICC}=.71, p<.001)$, but it was weaker for suicide plans $(\mathrm{ICC}=.23, p<.01)$ and was very poor for suicidal gestures $(\mathrm{ICC}=.01, n s)$.

Construct validity was demonstrated between the SITBI and the Schedule for Affective Disorders and Schizophrenia for School-Age Children-Present and Lifetime Version (K-SADS-PL; Kaufman, Birmaher, Brent, Rao, \& Ryan, 1997 as cited in Nock et al., 2007, p. 312). Results indicated good agreement between the SITBI and the KSADS-PL on the presence of a suicide attempt $(\kappa=0.65)$ and the presence of NSSI $(\kappa=$ 
$0.74)$, but less agreement on the presence of suicidal ideation $(\kappa=0.48)$. Construct validity was also demonstrated between the SITBI and the Beck Scale for Suicidal Ideation (A. T. Beck, Steer, \& Ranieri, 1988, as cited in Nock et al., 2007, p. 312). Results indicated good agreement on the presence of suicidal ideation $(\kappa=0.59)$. The SITBI has also been used with clinical adult populations (Cha, Najmi, Park, Finn, \& Nock, 2010; Nock et al., 2010) and undergraduate adult populations (Van Camp, Desmet, \& Verhaeghe, 2011).

\section{EDE-Q}

Second, select items from the Eating Disorder Questionnaire 6.0 (EDE-Q;

Fairburn \& Beglin, 2008) were used to assess the frequency of binge eating, purging, and restricting. The EDE-Q is a self-report questionnaire which measures thoughts and behaviors associated with eating. The EDE-Q consists of 22 items which are rated on a Likert scale, 6 frequency items which require a quantitative response, and 5 demographic items which require either a quantitative response or a yes or no answer. The 22 Likert items represent 4 subscales: eating concern, restraint, shape concern, and weight concern. These items ask respondents to indicate the number of days on which they have experienced the symptoms over the past 28 days. The six frequency items ask about the frequency of binge eating and compensatory behaviors. These items ask for either the number of times the individual engaged in the behaviors or the number of days on which the individual engaged in the behavior. The five demographic items ask about the respondent's height, weight, and menstrual history (Fairburn \& Beglin, 2008). In order to be consistent with the SITBI and this study's research questions, the time frame (28 days) was adjusted to obtain a lifetime frequency and a two-week frequency. Also for 
consistency, items will be answered with a quantitative, free-response rather than Likert scale. Higher numbers indicate higher frequencies of the behaviors.

Berg, Peterson, Frazier, and Crow (2012) reviewed 10 studies which examined the validity of the EDE-Q in a variety of different samples. Test-retest correlations for the four subscales ranged from 0.66 to 0.94 and test-retest correlations for the behavior frequency items ranged from 0.51 to 0.92 . The temporal stability of the EDE-Q was also demonstrated as the correlations between time 1 and time 2 administration (an average of 315 days between administrations) ranged from 0.42 to 0.69 and were all significant. Internal consistency of the subscales has been demonstrated with alphas ranging from 0.70 to 0.93 . Criterion validity has been demonstrated in that the EDE-Q discriminated between women with EDs and women without EDs and discriminated specifically between individuals who were obese and binge ate and individuals who were obese but did not binge eat. Additionally, convergent validity has been demonstrated in that selfreported frequency of behaviors on the EDE-Q aligns with the frequency of behaviors self-reported on daily food records.

\section{Self-Damaging Behaviors Questionnaire}

While not individual variables, it is important for the reader to note the language used to measure each of the behaviors composing the latent variable representing twoweek frequency of self-damaging behaviors and the latent variable representing lifetime frequency of self-damaging behaviors. Based on the items used to represent each behavior, the individual self-damaging behaviors were defined as follows.

Binge eating was defined as an individual eating what other people would regard as an unusually large amount of food (given the circumstances) while having a sense of 
having lost control over his/her eating (at the time that he/she was eating) (Fairburn \& Beglin, 2008). This behavior was measured using a combination of items \#13 and \#14 (lifetime frequency) and an adaptation of item \#15 (two-week frequency) from the Eating Disorder Exam Questionnaire 6.0 (EDE-Q).

Purging was defined as an individual making him/herself sick (vomit) as a means of controlling his/her weight or shape (Fairburn \& Beglin, 2008). This behavior was measured using two different adaptations of question \#16 (one for lifetime frequency and one for two-week frequency) from the EDE-Q.

Restricting involved two different behaviors. The first type of restricting was termed limiting and was defined as an individual deliberately trying to limit the amount of food he/she eats in order to maintain or achieve a significantly low body weight (a weight that is less than minimally recommended). Limiting was measured using two different adaptations (one for lifetime frequency and one for 2-week frequency) of question \#1 from the EDE-Q. The second type of restricting was termed fasting and was defined as an individual going for a long period of time ( 8 waking hours or more) without eating anything at all in order to influence his/her weight or shape (Fairburn \& Beglin, 2008). Fasting was measured using two different adaptations (one for lifetime frequency and one for two-week frequency) of question \#2 from the EDE-Q.

NSSI was defined as an individual purposely hurting him/herself (e.g., cutting or burning) without wanting to die (Nock et al., 2007). This behavior was measured using a combination of items \#116 and \#146 (lifetime frequency) and an adaptation of item \#149 (two-week frequency) of the Self-Injurious Thoughts and Behaviors Interview - Long Form (SITBI). 
Suicidal ideation was defined as an individual having thoughts of killing him/herself (Nock et al., 2007). This behavior was measured using item \#4 (lifetime frequency) and an adaptation of item \#7 (two-week frequency) of the SITBI.

A suicide attempt was defined as an individual having made an actual attempt to kill him/herself in which he/she had at least some intent to die (Nock et al., 2007). This behavior was measured using a combination of items \#84 and \#88 (lifetime frequency) and an adaptation of item \#91 (two-week frequency) of the SITBI.

Table 2 lists the original items and the resulting items (after adaptation and combination) used in the Self-damaging Behaviors Questionnaire. The first column lists the name of the behavior and the scale from which the item(s) representing the behavior were drawn. The second column lists the original wording of the item(s). The third column lists the wording of the item(s) as used in my study.

\section{Data Collection}

QuestionPro emailed potential respondents with an invitation to complete the survey. QuestionPro continued sending invitations until the desired sample size was reached. The first page of the survey consisted of an informed consent, which outlined the purposes of the study and identified potential risks and benefits of study participation. Potential respondents were informed that they could discontinue participation at any time. Contact information was provided for myself (the principal investigator) and my dissertation chair in case the potential participants had further questions about participation. 
Table 2

Adaptations and Combinations of Self-Damaging Behaviors Questionnaire Items

\begin{tabular}{|c|c|}
\hline $\begin{array}{l}\text { Behavior name } \\
\& \text { Instrument }\end{array}$ & Original Item(s) \\
\hline $\begin{array}{l}\text { Limiting } \\
\text { (Lifetime) }\end{array}$ & $\begin{array}{l}\text { EDE-Q \#1 } \\
\text { On how many of the past } 28 \\
\text { days have you been } \\
\text { deliberately trying to limit the } \\
\text { amount of food you eat to } \\
\text { influence your shape or } \\
\text { weight (whether or not you } \\
\text { have succeeded)? }\end{array}$ \\
\hline
\end{tabular}

Adapted/Combined Item

For the following questions, please

give your best estimate:

1. Over your lifetime, on how many

DAYS have you been deliberately

trying to limit the amount of food

you eat in order to maintain or

achieve a significantly low body

weight (a weight that is less than

minimally recommended)?

\begin{tabular}{|c|c|c|}
\hline $\begin{array}{l}\text { Limiting } \\
\text { (Two-week) }\end{array}$ & $\begin{array}{l}\text { EDE-Q \#1 } \\
\text { On how many of the past } 28 \\
\text { days have you been } \\
\text { deliberately trying to limit the } \\
\text { amount of food you eat to } \\
\text { influence your shape or } \\
\text { weight (whether or not you } \\
\text { have succeeded)? }\end{array}$ & $\begin{array}{l}\text { 2. How many DAYS in the past two } \\
\text { weeks? }\end{array}$ \\
\hline $\begin{array}{l}\text { Fasting } \\
\text { (Lifetime) }\end{array}$ & $\begin{array}{l}\text { EDE-Q \#2 } \\
\text { On how many of the past } 28 \\
\text { days have you gone for long } \\
\text { periods of time ( } 8 \text { waking } \\
\text { hours or more) without eating } \\
\text { anything at all in order to } \\
\text { influence your shape or } \\
\text { weight? }\end{array}$ & $\begin{array}{l}\text { 3. Over your lifetime, on how many } \\
\text { days have you gone for long periods } \\
\text { of time ( } 8 \text { waking hours or more) } \\
\text { without eating anything at all in } \\
\text { order to influence your shape or } \\
\text { weight? }\end{array}$ \\
\hline $\begin{array}{l}\text { Fasting } \\
\text { (Two-week) }\end{array}$ & $\begin{array}{l}\text { EDE-Q \#2 } \\
\text { On how many of the past } 28 \\
\text { days have you gone for long } \\
\text { periods of time ( } 8 \text { waking } \\
\text { hours or more) without eating } \\
\text { anything at all in order to } \\
\text { influence your shape or } \\
\text { weight? }\end{array}$ & $\begin{array}{l}\text { 4. How many days in the past two } \\
\text { weeks? }\end{array}$ \\
\hline
\end{tabular}


Table 2-Continued

\begin{tabular}{|c|c|c|}
\hline $\begin{array}{l}\text { Behavior name } \\
\& \text { Instrument }\end{array}$ & Original Item(s) & Adapted/Combined Item \\
\hline $\begin{array}{l}\text { Binge eating } \\
\text { (Lifetime) }\end{array}$ & $\begin{array}{l}\text { EDE-Q \#13: } \\
\text { Over the past } 28 \text { days, how } \\
\text { many times have you eaten } \\
\text { what other people would } \\
\text { regard as an unusually large } \\
\text { amount of food (given the } \\
\text { circumstances)? } \\
\text { EDE-Q \#14: } \\
\text {... On how many of these } \\
\text { times did you have a sense of } \\
\text { having lost control over your } \\
\text { eating (at the time that you } \\
\text { were eating)? } \\
\text { EDE-Q \#15 } \\
\text { Over the past } 28 \text { days, on } \\
\text { how many DAYS have such } \\
\text { episodes of overeating } \\
\text { occurred (i.e. you have eaten } \\
\text { an unusually large amount of } \\
\text { food and have had a sense of } \\
\text { loss of control at the time)? }\end{array}$ & $\begin{array}{l}\text { 5. Over your lifetime, on how many } \\
\text { days have episodes of binge eating } \\
\text { occurred? [Binge eating is defined as } \\
\text { eating what other people would } \\
\text { regard as an unusually large amount } \\
\text { of food (given the circumstances) and } \\
\text { having a sense of having lost control } \\
\text { over your eating (at the time that you } \\
\text { were eating).] }\end{array}$ \\
\hline $\begin{array}{l}\text { Binge eating } \\
\text { (Two-week) }\end{array}$ & $\begin{array}{l}\text { EDE-Q \#15 } \\
\text { Over the past } 28 \text { days, on } \\
\text { how many DAYS have such } \\
\text { episodes of overeating } \\
\text { occurred (i.e. you have eaten } \\
\text { an unusually large amount of } \\
\text { food and have had a sense of } \\
\text { loss of control at the time)? }\end{array}$ & $\begin{array}{l}6 . \text { How many days in the past two } \\
\text { weeks? }\end{array}$ \\
\hline $\begin{array}{l}\text { Purging } \\
\text { (Lifetime) }\end{array}$ & $\begin{array}{l}\text { EDE-Q \#16 } \\
\text { Over the past } 28 \text { days, how } \\
\text { many times have you made } \\
\text { yourself sick (vomit) as a } \\
\text { means of controlling your } \\
\text { shape or weight? }\end{array}$ & $\begin{array}{l}\text { 7. Over your lifetime, how many } \\
\text { TIMES have you made yourself } \\
\text { sick (vomit) as a means of } \\
\text { controlling your shape or weight? }\end{array}$ \\
\hline
\end{tabular}


Table 2-Continued

\begin{tabular}{|c|c|c|}
\hline $\begin{array}{l}\text { Behavior name } \\
\& \text { Instrument }\end{array}$ & Original Item(s) & Adapted/Combined Item \\
\hline $\begin{array}{l}\text { Purging } \\
\text { (Two-week) }\end{array}$ & $\begin{array}{l}\text { EDE-Q \#16 } \\
\text { Over the past } 28 \text { days, how } \\
\text { many times have you made } \\
\text { yourself sick (vomit) as a } \\
\text { means of controlling your } \\
\text { shape or weight? }\end{array}$ & $\begin{array}{l}\text { 8. How many TIMES in the past } \\
\text { two weeks? }\end{array}$ \\
\hline $\begin{array}{l}\text { Nonsuicidal } \\
\text { self-injury } \\
\text { (Lifetime) }\end{array}$ & $\begin{array}{l}\text { SITBI \#116 } \\
\text { Have you ever had thoughts } \\
\text { of purposely hurting yourself } \\
\text { without wanting to die? (for } \\
\text { example, cutting or burning) } \\
\text { SITBI \#146 } \\
\text { How many times in your life } \\
\text { have you engaged in NSSI? }\end{array}$ & $\begin{array}{l}\text { 9. How many times in your life have } \\
\text { you engaged in nonsuicidal self- } \\
\text { injury? [Nonsuicidal self-injury is } \\
\text { defined as purposely hurting yourself } \\
\text { without wanting to die (for example, } \\
\text { cutting or burning).] }\end{array}$ \\
\hline $\begin{array}{l}\text { Nonsuicidal } \\
\text { self-injury } \\
\text { (Two-week) }\end{array}$ & $\begin{array}{l}\text { SITBI \#149 } \\
\text { How many times in the past } \\
\text { week? }\end{array}$ & $\begin{array}{l}\text { 10. How many times in the past two } \\
\text { weeks? }\end{array}$ \\
\hline $\begin{array}{l}\text { Suicidal } \\
\text { ideation } \\
\text { (Lifetime) }\end{array}$ & $\begin{array}{l}\text { SITBI \#4 } \\
\text { During how many separate } \\
\text { times in your life have you } \\
\text { had thoughts of killing } \\
\text { yourself? (Please give your } \\
\text { best estimate.) }\end{array}$ & $\begin{array}{l}\text { 11. How many separate times in } \\
\text { your life have you had thoughts of } \\
\text { killing yourself? }\end{array}$ \\
\hline $\begin{array}{l}\text { Suicidal } \\
\text { ideation } \\
\text { (Two-week) }\end{array}$ & $\begin{array}{l}\text { SITBI \#7 } \\
\text { How many separate times in } \\
\text { the past week? }\end{array}$ & $\begin{array}{l}\text { 12. How many separate times in the } \\
\text { past two weeks? }\end{array}$ \\
\hline $\begin{array}{l}\text { Suicide } \\
\text { attempts } \\
\text { (Lifetime) }\end{array}$ & $\begin{array}{l}\text { SITBI \#84 } \\
\text { Have you ever made an } \\
\text { actual attempt to kill yourself } \\
\text { in which you had at least } \\
\text { some intent to die? } \\
\text { SITBI \#88 } \\
\text { How many suicide attempts } \\
\text { have you made in your } \\
\text { lifetime? }\end{array}$ & $\begin{array}{l}\text { 13. How many suicide attempts } \\
\text { have you made in your lifetime? [A } \\
\text { suicide attempt is defined as an actual } \\
\text { attempt to kill yourself in which you } \\
\text { had at least some intent to die.] }\end{array}$ \\
\hline
\end{tabular}


Table 2-Continued

\begin{tabular}{lll}
\hline $\begin{array}{l}\text { Behavior name } \\
\text { \& Instrument }\end{array}$ & Original Item(s) & Adapted/Combined Item \\
\hline $\begin{array}{ll}\text { Suicide } \\
\text { attempts }\end{array}$ & $\begin{array}{l}\text { SITBI \#91 } \\
\text { (Two-week) }\end{array}$ & $\begin{array}{l}\text { the past week? } \\
\text { tho How many have you made in }\end{array}$ \\
\hline
\end{tabular}

Given the sensitive nature of the topics addressed in the surveys, the informed consent explicitly listed the topics which will be addressed in the surveys (namely, current experiences of anxiety or depression as well as current and past history of eating behaviors, NSSI, suicidal ideation, and suicide attempts). The use of surveys to address similar topics in the behavioral sciences is a widespread practice, so harm from participation was not anticipated (Whitlock, Pietrusza, \& Purington, 2013). However, phone numbers for national helplines were listed as part of the informed consent in case completion of the survey elicited the participant desiring to seek further help or consultation regarding the thoughts and behaviors surveyed (these numbers were also listed on the second to last screen of the survey). Since the sample was drawn from a wide geographic region, referrals to specific mental health professionals were not possible to include. Instead, the potential participants were informed of a phone service which can offer local referrals. Participants clicked a button to acknowledge that they had read and understood the information contained in the informed consent and that by answering the survey questions, they gave their consent to participation in the study.

After the informed consent, participants were asked two screening questions. The first was, "Are you 18 years or older?" If the answer was no, participants were immediately routed to the last screen of the survey which thanked them for their 
participation (individuals who did not complete the full survey were not awarded points, per QuestionPro policy). The second screening question was, "Have you experienced at least one of the following over the course of your lifetime: binge eating, nonsuicidal selfinjury (e.g., cutting or burning), purging (i.e., making yourself vomit), very restricted eating, suicidal ideation, or a suicide attempt?" Again, if the answer was no, participants were immediately routed to the last screen of the survey which thanked them for their participation. When initiating the study, it was unknown what percentage of the general population would endorse the second screening question. It was roughly estimated that $30 \%$ of the population might endorse the question, given the prevalence of individual behaviors included in the study. The actual incidence rate is reported with the results.

Next, participants completed a brief demographic survey gathering information including age, gender, ethnicity, and income. The participants then completed the ERS, followed by the LHS, sDTS, select items from the DASS-21, and the Self-damaging Behaviors Questionnaire. A button was available to discontinue the survey at any time throughout the survey. At the end of the survey, a final screen thanked the respondents for their participation and informed them that QuestionPro had added points to their account. QuestionPro allows participants to receive points in exchange for completed surveys. These accumulated points can be redeemed for $\$ 10.00$ gift certificates to popular stores and restaurants. QuestionPro estimated that each participant would earn the equivalent of $\$ 2.00$ in points for completing my survey.

\section{Treatment of Data}

All survey answers were kept confidential. I used QuestionPro's Respondent Anonymity Assurance to ensure that respondent identifying information was not linked to 
survey responses. Respondent Anonymity Assurance assigned a random code (which is not linked to identifying information) to each completed survey. QuestionPro did not release to anyone any potentially identifying information about respondents, except for IP address. I deleted the IP addresses upon receipt of the data. QuestionPro kept a record of who completed the survey in order to ensure (a) that the same individual did not take the same survey twice and (b) that the individual was assigned points for the completion of the survey. However, this record was not linked to survey responses.

Once the data was downloaded from QuestionPro, the IP addresses were deleted and the resulting data was saved in SPSS. An identification number in SPSS allowed for the data to be distinguished by subject, despite the lack of identifiable information. The data was stored in a password-protected laptop and a backup was on an external USB drive (stored by the principal investigator). Only committee members and I had access to the raw data. Survey data will be stored for at least three years, per Institutional Review Board policy. After the minimum of 3 years, data will be securely deleted after the publication process is completed.

\section{Data Analysis}

I analyzed my data using SEM in order to determine the strength of the relationship between the variables. Structural equation modeling was a good fit for my study since it allowed me to examine a model developed based on theory. Structural equation modeling also allowed for the analysis of both observed and latent variables. Compared to other regression methods, SEM is strengthened by its ability to account for measurement error in independent variables. It also allows for the simultaneous evaluation of multiple independent and dependent variables. 
Before assessing model fit, I conducted data screening both by case and by variable (Gaskin, 2012, 2013). In case screening, I screened for missing data, unengaged data (i.e., answering items without regard for the content of the question), and outliers. For missing data, if there was data missing for less than $10 \%$ of the variables for any single case, the values were imputed (in variable screening). If there was data missing for $10 \%$ or more of the variables, the case was deleted. Cases with unengaged data (any case with a standard deviation of zero among the scores on the Likert-scale items) were deleted. Cases with a standard deviation of less than .5 on the Likert-scale items were visually scanned for clear disengagement (e.g., no variance of answers within a single instrument). For outliers, only the lifetime frequency of self-damaging behaviors and age were screened (since the rest of the data results from limited-response items). When outliers were identified to the point that interpretation of results would be hindered, the next lowest value below the outlier which was not an outlier itself was imputed for the outlier.

In variable screening, I screened for missing values, skewness, and kurtosis (Gaskin, 2012, 2013). For missing values, values for items rated on an ordinal scale were imputed with the sample's median value. Values for items rated on a continuous scale were imputed with the sample's mean value. Then, skewness (for continuous-scale items) and kurtosis (for continuous-scale and ordinal-scale items) were examined. Items were considered to demonstrate skewness or kurtosis if the absolute value of the skewness or kurtosis was greater than 3 (Gaskin, 2016). While SEM is rather robust to both skewness and kurtosis, any variables demonstrating strong skewness or kurtosis were dropped (ordinal) or transformed (continuous). 
Descriptive statistics obtained included means, standard deviations, and correlations. Then, internal validity statistics were calculated for the instrumentation. Then, the structural model was examined.

Rather than a strictly confirmatory approach, I took a model generating approach to model analysis (Byrne, 2010). When the initial model was not a good fit for the data, it was respecified based on the data. As the goal was to find a model which is both theoretically meaningful and well-fit to the data, introduction of respecifications was prioritized based on theory. The respecified model would then be re-estimated. The fit of the model was evaluated on the basis of the chi-square test $(p \geq .05)$, goodness-of-fit index (GFI; $\geq .90)$, normed fit index (NFI; $\geq .95)$, comparative fit index (CFI; $\geq .95)$, and the root mean square error of approximation (RMSEA; $\leq .06$; Cut-off values were determined according to the recommendations of Hooper, Coughlan, \& Mullen, 2008). It is common for models with large sample sizes to be well fit without a nonsignificant chisquare (Byrne, 2010; Raykov \& Marcoulides, 2006). Therefore, it was anticipated that the models might instead be evaluated based on the fit indexes.

Of note, my results speak to the degree of fit observed between my resulting SEM models and the data of the current sample. It will always be possible that different models (including different variables or the same variables arranged in a different configuration) would be a better fit for the data. In behavioral research, models which most accurately represent human behavior are likely to include a large number of variables. However, strong models for the relationship between key variables will serve as a foundation for future (perhaps more complex) models. 


\section{Budget}

The total budget for this project was $\$ 18,700$. This budget included $\$ 17,000$ for dissertation course credits, $\$ 1,200$ for 300 responses through QuestionPro, and $\$ 500$ for editing and printing. The project was self-funded by the primary researcher.

\section{Summary}

In this chapter, the research methodology for the present study was described. This study used SEM to examine individual difference factors in DT in the context of self-damaging behaviors. Data was collected through surveys conducted through an online platform, QuestionPro. The sample consisted of individuals 18 years and older who had a lifetime history of experiencing binge eating, purging, restricting, NSSI, suicidal ideation, or suicide attempts. The research variables were defined in this chapter and the instruments used to measure the variables were outlined. The research questions were proposed and the data analysis procedures were described. Chapter 4 will present the results of the research, and Chapter 5 will discuss the implications of the results. 


\section{CHAPTER 4}

\section{RESULTS}

\section{Introduction}

The purpose of this research was to test two models of individual difference factors in DT, one in the context of lifetime frequency and one in the context of twoweek frequency of self-damaging behaviors. In particular, I sought to investigate the role of two potential individual difference factors in DT - emotional reactivity and learned helplessness. Structural equation modeling was used to determine whether the relationships between the variables proposed by the models were aligned with sample data. This chapter will present a description of this study's sample, a description of the study's variables, and the results of the SEM analysis.

\section{Description of the Sample}

A total of 694 individuals clicked on the link to my survey. However, some of these cases were excluded from the data analysis because the participant did not meet my study's criteria or the participant chose to discontinue the survey before completion. Of the individuals who viewed my survey, 585 consented to the informed consent. Of those who consented to the informed consent, 306 (52\% of those who completed the consent) completed the whole survey. This was 6 respondents more than the proposed 300 responses. 


\section{Discontinued Responses}

That means that 279 individuals did not meet my study's criteria or chose to discontinue. Of these, $225(81 \%)$ were automatically terminated for not meeting study criteria (i.e., 218 [97\%] did not endorse a history of self-damaging behaviors, seven [3\%] did not endorse being age 18 or older). These responses were deleted from the data set. The remaining 54 (19\%) individuals discontinued the survey before reaching the end. This was interpreted as the individuals revoking their consent to participate in the study, so their responses were also deleted from the data set.

While not central to the hypotheses of this study, it is perhaps useful to the extant literature to note the percentage of respondents who endorsed a lifetime history of at least one of the self-damaging behaviors. A total of 563 respondents answered the second screener question which asked "Have you experienced at least one of the following over the course of your lifetime: binge eating, nonsuicidal self-injury (e.g., cutting or burning), purging (i.e., making yourself vomit), very restricted eating, suicidal ideation, or a suicide attempt?" Of these, 345 responded "yes". Thus, $61.28 \%$ of initial respondents affirmed a lifetime history of at least one of these self-damaging behaviors.

\section{Data Screening}

Among the 306 completed surveys, an additional 29 were removed from the data set after manual review in data screening. Of these 29 , one was removed for entering an age less than 18 , despite endorsing being over age 18 on the screening question. Another four were removed for not endorsing any of the individual self-damaging behaviors, despite endorsing a history of self-damaging behaviors on the screening question. This 
was possible due to the Self-damaging Behavior Questionnaire specifying the criteria for self-damaging behaviors more specifically than the brief screener question (e.g., the screener question listed "binge eating," whereas the Self-damaging Behavior Questionnaire further specified binge eating as involving eating an unusually large amount of food and having a sense of having lost control). Another 23 indicated a higher frequency of one of the self-damaging behaviors over the past two weeks than over their lifetime, which is logically impossible. So, these responses were considered unengaged data and were removed. The standard deviation of a respondent's answers across all instruments was calculated and any responses with a standard deviation less than five were visually inspected for engagement. This resulted in one additional response being removed as unengaged.

After these cases were removed from the data set, 277 surveys remained which could be included in the data analysis. Within these 277 , two respondents endorsed engaging in self-damaging behaviors on more days over their lifetime than the number of days they had been alive. While this is logically impossible, it was interpreted as potentially exaggerated data rather than as disengaged data. The strength of the values was interpreted as likely reflecting true extremity of the individual's experience (e.g. a 20 year old who indicated 8000 days of restricting has likely truly experienced a high number of days of restricting). So, the value was replaced with the respondent's age multiplied by 365 as a rough estimate of the highest value which could have been true.

\section{Participants Description}

Demographic information about the sample is presented in Table 3. Demographic categories which were not endorsed by respondents are not included. The most frequently 
Table 3

Respondents' Demographic Characteristics

\begin{tabular}{|c|c|c|}
\hline Demographic & $N$ & $\%$ \\
\hline \multicolumn{3}{|l|}{ Age } \\
\hline & & \\
\hline \multicolumn{3}{|l|}{ Frequency by groups } \\
\hline $18-29$ & 85 & 30.7 \\
\hline $30-39$ & 104 & 37.5 \\
\hline $40-49$ & 53 & 19.1 \\
\hline $50-59$ & 25 & 9.0 \\
\hline $60+$ & 10 & 3.6 \\
\hline \multicolumn{3}{|l|}{ Ethnicity } \\
\hline African American & 16 & 5.8 \\
\hline American Indian & 3 & 1.1 \\
\hline Asian American & 4 & 1.4 \\
\hline Caucasian American & 227 & 81.9 \\
\hline Latino/a & 18 & 6.5 \\
\hline Multiracial & 8 & 2.9 \\
\hline Other & 1 & 0.4 \\
\hline \multicolumn{3}{|l|}{ Gender } \\
\hline Female & 172 & 62.1 \\
\hline Male & 105 & 37.9 \\
\hline \multicolumn{3}{|l|}{ Income Range } \\
\hline$\$ 0$ to $\$ 9,999$ & 7 & 2.5 \\
\hline$\$ 10,000$ to $\$ 24,999$ & 23 & 8.3 \\
\hline$\$ 25,000$ to $\$ 49,999$ & 64 & 23.1 \\
\hline$\$ 50,000$ to $\$ 74,999$ & 68 & 24.5 \\
\hline$\$ 75,000$ to $\$ 99,999$ & 53 & 19.1 \\
\hline$\$ 100,000$ to $\$ 124,999$ & 28 & 10.1 \\
\hline$\$ 125,000$ to $\$ 149,999$ & 12 & 4.3 \\
\hline$\$ 150,000+$ & 22 & 7.9 \\
\hline \multicolumn{3}{|l|}{ Marital Status } \\
\hline Single, never married & 92 & 33.2 \\
\hline Engaged or married & 155 & 56.0 \\
\hline Separated or divorced & 26 & 9.4 \\
\hline Widowed & 3 & 1.1 \\
\hline Widowed and engaged or married & 1 & 0.4 \\
\hline
\end{tabular}


endorsed participant characteristics were Caucasian American female, age 36, who was engaged or married, with an income between $\$ 50,000$ and $\$ 74,999$. Compared to the QuestionPro Survey Analytics panel, my sample had similar age and ethnic representation. However, my sample had a greater representation of females $(62 \%)$ than the Survey Analytics panel (53\%).

\section{Instrument Reliability}

Table 4 lists the Cronbach's alpha for each of the instruments used in this study. All instruments demonstrated acceptable (over .7) levels of internal reliability in this study. This suggests that the scales each measured a single latent construct. The DASS 21-Depression, the ERS, and the sDTS demonstrated excellent internal reliability (over .9). The DASS 21- Anxiety and the LHS demonstrated good internal reliability (over .8). The Self-damaging Behaviors Questionnaire demonstrated the lowest, but still acceptable, internal reliability at .720 . Table 4 also lists the Hancock coefficient (Hancock \& Mueller, 2001) for each of the instruments. All instruments demonstrated acceptable (over .8) levels of maximal reliability in this study.

Table 4

Internal Reliability

\begin{tabular}{lcc}
\hline Instrument & $\alpha$ & $H$ \\
\hline DASS 21- Anxiety & .835 & .862 \\
DASS 21- Depression & .902 & .975 \\
SDTS & .919 & .967 \\
ERS-6 Item Reduction & .871 & .932 \\
LHS & .851 & .950 \\
Self-damaging Behaviors Questionnaire & .720 & .977 \\
\hline
\end{tabular}




\section{Emotion Reactivity Scale Item Reduction}

As proposed, the models were tested using the subscale scores (rather than the individual items) as the observed variables for the LHS and the sDTS. This was done in order for the models to be identified. Since the extant literature does not support subscales within the ERS, the 6 items with the highest regression weights onto the latent variable of emotional reactivity were included in the model analyses. The items selected based on preliminary SEM were items 5, 11, 14, 15, 19, and 20. The internal reliability of the scale created by these 6 items alone demonstrated good internal reliability at $\alpha=.871$. However, it should be noted that the item-limited scale demonstrated lower internal reliability than the full scale at $\alpha=.939$.

\section{Variables Description}

\section{Normality}

As proposed, all variables were screened for kurtosis and the continuous variables were screened for skewness. Age, anxiety, depression, emotional reactivity, learned helplessness, and DT were within limits ( -3 to 3 ) for skewness and kurtosis. Initially, all of the items on the Self-damaging Behaviors Questionnaire, except for two-week limiting, demonstrated high kurtosis. Similarly, all of the self-damaging behaviors items, except for two-week limiting, restricting, and binging, were highly skewed. When qualitatively considering the variables, the kurtosis is explained by a large amount of respondents answering near zero (small distribution) in response to frequency questions. The positive skewness is explained by a large amount of respondents indicating low frequency of self-damaging behaviors. For example, two-week suicide attempts was the 
item with the highest skewness and kurtosis. As is consistent with lived experience, few respondents endorsed a suicide attempt in the past two weeks (see endorsement of selfdamaging behaviors below) which resulted in a small distribution with the most frequent response falling under the mean score.

Though SEM is resilient to skewness and kurtosis, the level exhibited in the items from the Self-damaging Behaviors Questionnaire was too strong to move forward with SEM. So, the items from the Self-damaging Behaviors Questionnaire were natural log transformed. After log transformation, the skewness of all items was within -3 to 3 except for two-week purging (3.51), two-week NSSI (4.15), and two-week attempts (13.84). The kurtosis of all items was within -3 to 3 except for two-week purging (12.11), two-week NSSI (17.32), two-week ideation (6.78), and two-week attempts (207.94). Items not meeting criteria for skewness and kurtosis were carefully examined in SEM.

\section{Variable Means and Standard Deviations}

After the log transformation of items from the Self-damaging Behaviors Questionnaire, clear outliers were removed from 8 of the variables and were replaced with the next highest value to reduce their influence. After removal of outliers and transformation, the variables more closely represented the normal distribution and were appropriate for evaluation using SEM. Variable means and standard deviations are presented in Table 5. In Table 5, the original mean and standard deviation are listed for each variable, with the log-transformed mean and standard deviation listed in parentheses. 
Table 5

Variable Means and Standard Deviations

\begin{tabular}{lccc}
\hline Variable & Scale Range & Mean & $S D$ \\
\hline DASS-21, item 2 (Anxiety) & $0-3$ & 0.95 & 0.91 \\
DASS-21, item 4 (Anxiety) & $0-3$ & 0.89 & 0.90 \\
DASS-21, item 6 (Anxiety) & $0-3$ & 0.82 & 0.88 \\
DASS-21, item 9 (Anxiety) & $0-3$ & 1.23 & 1.03 \\
DASS-21, item 15 (Anxiety) & $0-3$ & 1.05 & 0.99 \\
DASS-21, item 19 (Anxiety) & $0-3$ & 1.20 & 0.99 \\
DASS-21, item 20 (Anxiety) & $0-3$ & 1.06 & 0.99 \\
DASS-21, item 3 (Depression) & $0-3$ & 0.80 & 0.85 \\
DASS-21, item 5 (Depression) & $0-3$ & 1.34 & 0.97 \\
DASS-21, item 10 (Depression) & $0-3$ & 1.21 & 0.98 \\
DASS-21, item 13 (Depression) & $0-3$ & 1.34 & 0.90 \\
DASS-21, item 16 (Depression) & $0-3$ & 1.19 & 0.97 \\
DASS-21, item 17 (Depression) & $0-3$ & 1.13 & 1.05 \\
DASS-21, item 21 (Depression) & $0-3$ & 0.93 & 1.06 \\
ERS, item 5 & $0-4$ & 1.95 & 1.19 \\
ERS, item 11 & $0-4$ & 1.76 & 1.24 \\
ERS, item 14 & $0-4$ & 1.68 & 1.22 \\
ERS, item 15 & $0-4$ & 1.52 & 1.19 \\
ERS, item 19 & $0-4$ & 2.12 & 1.21 \\
ERS, item 20 & $0-4$ & 1.73 & 1.24 \\
LHS (Ability-Inability) & $2-8$ & 4.80 & 1.07 \\
LHS (Choice) & $2-8$ & 4.69 & 0.99 \\
LHS (Global-Specific) & $5-20$ & 11.78 & 2.33 \\
LHS (Internal-External) & $5-20$ & 11.62 & 2.38 \\
LHS (Stable-Unstable) & $6-24$ & 13.36 & 2.70 \\
sDTS (Absorption) & $1-5$ & 2.73 & 1.05 \\
sDTS (Appraisal) & $1-5$ & 2.97 & 0.89 \\
sDTS (Regulation) & $1-5$ & 2.77 & 0.97 \\
sDTS (Tolerance) & 2.79 & 0.98 \\
& & &
\end{tabular}


Table 5-Continued

\begin{tabular}{lcrr}
\hline Variable & $\begin{array}{c}\text { Observed } \\
\text { Range }\end{array}$ & Mean (lnmean) & \multicolumn{1}{c}{$S D(\ln S D)$} \\
\hline Lifetime Binging & $0-13,140$ & $397.80(3.01)$ & $1898.11(2.51)$ \\
Two-week Binging & $0-14$ & $1.49(0.57)$ & $2.59(0.76)$ \\
Lifetime Purging & $0-2,000$ & $56.35(1.11)$ & $260.41(1.81)$ \\
Two-week Purging & $0-5$ & $0.15(0.08)$ & $0.57(0.29)$ \\
Lifetime Limiting & $0-15,000$ & $711.49(3.83)$ & $2127.91(2.77)$ \\
Two-week Limiting & $0-14$ & $3.33(0.97)$ & $4.28(1.00)$ \\
Lifetime Fasting & $0-10,000$ & $319.23(2.79)$ & $1096.63(2.57)$ \\
Two-week Fasting & $0-14$ & $1.61(0.55)$ & $3.05(0.81)$ \\
Lifetime NSSI & $0-2,000$ & $37.88(1.24)$ & $207.66(1.64)$ \\
Two-week NSSI & $0-8$ & $.21(0.09)$ & $.94(0.35)$ \\
Lifetime Suicidal Ideation & $0-10,000$ & $255.39(2.06)$ & $1283.05(2.12)$ \\
Two-week Suicidal Ideation & $0-30$ & $1.29(0.32)$ & $4.53(0.73)$ \\
Lifetime Suicide Attempts & $0-10$ & $.64(0.29)$ & $1.49(0.54)$ \\
Two-week Suicide Attempts & $0-8$ & $.05(0.03)$ & $.52(0.30)$ \\
\hline
\end{tabular}

Endorsement of Self-Damaging Behaviors

Table 6 lists the percentage of respondents who endorsed each of the selfdamaging behaviors. Overall, endorsement of individual self-damaging behaviors over the lifetime ranged from $27.8 \%$ to $77.3 \%$ of the sample. Endorsement of individual selfdamaging behaviors over the last two weeks ranged from $1.8 \%$ to $54.5 \%$ of the sample. Among the self-damaging behaviors, limiting was the most frequently endorsed and suicide attempts were the least frequently endorsed. 
Table 6

Respondents Endorsing Each Self-Damaging Behavior

\begin{tabular}{lrr}
\hline Variable & $n(277)$ & $\%$ \\
\hline Lifetime Binging & 198 & 71.5 \\
Two-week Binging & 117 & 42.2 \\
Lifetime Purging & 106 & 38.3 \\
Two-week Purging & 25 & 9.0 \\
Lifetime Limiting & 214 & 77.3 \\
Two-week Limiting & 151 & 54.5 \\
Lifetime Fasting & 194 & 70.0 \\
Two-week Fasting & 101 & 36.5 \\
Lifetime NSSI & 137 & 49.5 \\
Two-week NSSI & 23 & 8.3 \\
Lifetime Suicidal Ideation & 204 & 73.6 \\
Two-week Suicidal Ideation & 62 & 22.4 \\
Lifetime Suicide Attempts & 77 & 27.8 \\
Two-week Suicide Attempts & 5 & 1.8 \\
\hline
\end{tabular}

Variable Description by Demographic Characteristics

Table 7 presents the means and standard deviations for DT, emotional reactivity, and learned helplessness on the basis of age group, sex, ethnicity, marital status, and income level. While examining group differences was outside the scope of this study, a few potential trends based on visual inspection will be noted throughout this description of sample demographics. Here there appears to be a trend that females reported higher mean learned helplessness, higher mean emotional reactivity, and lower mean distress tolerance than males. 
Table 7

Emotional Reactivity, Learned Helplessness, and DT by Demographic

Characteristics

\begin{tabular}{|c|c|c|c|c|c|c|c|}
\hline \multirow[b]{2}{*}{ Characteristics } & \multirow[b]{2}{*}{$n$} & \multicolumn{2}{|c|}{$\begin{array}{l}\text { Emotional } \\
\text { reactivity }\end{array}$} & \multicolumn{2}{|c|}{$\begin{array}{c}\text { Learned } \\
\text { helplessness }\end{array}$} & \multicolumn{2}{|c|}{$\begin{array}{l}\text { Distress } \\
\text { tolerance }\end{array}$} \\
\hline & & $M$ & $S D$ & $M$ & $S D$ & $M$ & $S D$ \\
\hline \multicolumn{8}{|l|}{ Age } \\
\hline $18-29$ & 85 & 17.88 & 5.90 & 47.68 & 7.42 & 2.59 & 0.75 \\
\hline $30-39$ & 104 & 17.08 & 5.30 & 46.11 & 7.41 & 2.74 & 0.81 \\
\hline $40-49$ & 53 & 16.26 & 5.76 & 46.13 & 6.86 & 2.96 & 0.91 \\
\hline $50-59$ & 25 & 14.04 & 5.47 & 43.56 & 7.12 & 3.25 & 0.88 \\
\hline $60+$ & 10 & 13.30 & 4.97 & 43.20 & 8.99 & 3.50 & 0.63 \\
\hline \multicolumn{8}{|l|}{ Sex } \\
\hline Female & 172 & 17.27 & 5.87 & 47.42 & 7.29 & 2.75 & 0.83 \\
\hline Male & 105 & 15.91 & 5.28 & 44.36 & 7.21 & 2.92 & 0.86 \\
\hline \multicolumn{8}{|l|}{ Ethnicity } \\
\hline African American & 16 & 17.75 & 5.89 & 45.63 & 6.77 & 2.75 & 0.57 \\
\hline Latino/a American & 18 & 16.72 & 6.02 & 42.72 & 7.28 & 2.78 & 0.98 \\
\hline American Indian & 3 & 14.67 & 2.89 & 44.33 & 11.24 & 3.28 & 0.45 \\
\hline Asian American & 4 & 14.25 & 4.35 & 47.50 & 7.90 & 3.16 & 0.60 \\
\hline Caucasian American & 227 & 16.82 & 5.72 & 46.66 & 7.46 & 2.80 & 0.85 \\
\hline Multiracial & 8 & 15.75 & 5.50 & 44.88 & 5.05 & 2.91 & 1.10 \\
\hline \multicolumn{8}{|l|}{ Marital Status } \\
\hline Single, never married & 92 & 17.25 & 5.39 & 47.52 & 7.80 & 2.62 & 0.75 \\
\hline Engaged or Married & 155 & 16.51 & 5.95 & 45.57 & 7.29 & 2.88 & 0.89 \\
\hline Separated or Divorced & 26 & 16.58 & 5.24 & 45.23 & 5.71 & 3.12 & 0.80 \\
\hline Widowed & 3 & 15.33 & 6.66 & 47.67 & 8.96 & 2.88 & 0.42 \\
\hline \multicolumn{8}{|l|}{ Income } \\
\hline$\$ 0$ to $\$ 9,999$ & 7 & 15.57 & 4.12 & 52.29 & 5.56 & 2.83 & 0.97 \\
\hline$\$ 10,000$ to $\$ 24,999$ & 23 & 19.09 & 4.73 & 49.57 & 7.26 & 2.65 & 0.70 \\
\hline$\$ 25,000$ to $\$ 49,999$ & 64 & 18.00 & 6.63 & 47.03 & 8.03 & 2.57 & 0.87 \\
\hline$\$ 50,000$ to $\$ 74,999$ & 68 & 17.32 & 5.09 & 46.32 & 6.75 & 2.68 & 0.77 \\
\hline$\$ 75,000$ to $\$ 99,999$ & 53 & 15.04 & 5.74 & 45.02 & 6.77 & 3.00 & 0.81 \\
\hline$\$ 100,000$ to $\$ 124,999$ & 28 & 15.11 & 5.19 & 44.36 & 6.78 & 3.09 & 0.89 \\
\hline$\$ 125,000$ to $\$ 149,999$ & 12 & 16.25 & 5.74 & 42.17 & 8.95 & 3.14 & 0.79 \\
\hline$\$ 150,000$ to $\$ 174,999$ & 8 & 14.25 & 4.77 & 46.38 & 6.67 & 3.41 & 0.83 \\
\hline$\$ 175,000$ to $\$ 199,999$ & 6 & 15.33 & 3.67 & 45.50 & 7.71 & 3.00 & 0.69 \\
\hline$\$ 200,000+$ & 8 & 17.88 & 5.62 & 46.25 & 9.42 & 2.84 & 1.10 \\
\hline
\end{tabular}


Table 8 presents the log-transformed means and standard deviations for the twoweek frequency of self-damaging behaviors on the basis of demographic characteristics. Table 9 presents the log-transformed means and standard deviations for the lifetime frequency of self-damaging behaviors on the basis of demographic characteristics. There appears to be a trend in which females have higher means on two-week and lifetime frequency of all self-damaging behaviors, except for two-week frequency of purging. There also appears to be a trend in both two-week and lifetime frequency of NSSI in which younger participants report the highest frequency, with frequency decreasing as age increases.

\section{Zero-Order Correlations}

Table 10 presents zero order (Pearson) correlations. As anticipated, anxiety, depression, DT, emotional reactivity, and learned helplessness were all significantly correlated with each other. The correlations were in the moderate range, which suggests that each variable measured a distinct construct.

The significant correlations that existed between variables were all in the anticipated directions. That is, variables which were significantly correlated with DT were all negatively correlated (e.g., higher emotional reactivity was correlated with lower distress tolerance). The rest of the significant correlations between variables were positive correlations (e.g., higher emotional reactivity was correlated with higher learned helplessness). 
Table 8

Two-Week Frequency of Self-Damaging Behaviors by Demographic Characteristics*

\begin{tabular}{|c|c|c|c|c|c|c|c|c|c|}
\hline \multirow[b]{2}{*}{ Characteristics } & \multirow[b]{2}{*}{$n$} & \multicolumn{2}{|c|}{ Limiting } & \multicolumn{2}{|c|}{ Fasting } & \multicolumn{2}{|c|}{ Binging } & \multicolumn{2}{|c|}{ Purging } \\
\hline & & $M$ & $S D$ & $M$ & $S D$ & $M$ & $S D$ & $M$ & $S D$ \\
\hline \multicolumn{10}{|l|}{ Age } \\
\hline 18-29 & 85 & 1.14 & 1.02 & 0.64 & 0.87 & 0.56 & 0.77 & 0.11 & 0.33 \\
\hline $30-39$ & 104 & 0.79 & 0.96 & 0.52 & 0.83 & 0.55 & 0.70 & 0.10 & 0.32 \\
\hline $40-49$ & 53 & 1.09 & 1.02 & 0.54 & 0.78 & 0.60 & 0.80 & 0.06 & 0.22 \\
\hline $50-59$ & 25 & 1.07 & 0.93 & 0.44 & 0.63 & 0.46 & 0.71 & 0.03 & 0.14 \\
\hline $60+$ & 10 & 0.59 & 1.02 & 0.29 & 0.63 & 0.92 & 1.09 & 0.00 & --- \\
\hline \multicolumn{10}{|l|}{ Sex } \\
\hline Female & 172 & 1.09 & 1.02 & 0.60 & 0.85 & 0.63 & 0.79 & 0.10 & 0.31 \\
\hline Male & 105 & 0.77 & 0.93 & 0.46 & 0.73 & 0.47 & 0.69 & 0.67 & 0.24 \\
\hline \multicolumn{10}{|l|}{ Ethnicity } \\
\hline African American & 16 & 1.41 & 0.96 & 1.12 & 0.97 & 1.02 & 0.92 & 0.22 & 0.52 \\
\hline Latino/a American & 18 & 0.95 & 1.03 & 0.51 & 0.92 & 0.37 & 0.62 & 0.39 & 0.16 \\
\hline American Indian & 3 & 2.13 & 0.31 & 1.68 & 0.78 & 1.50 & 1.34 & 0.00 & --- \\
\hline Asian American & 4 & 0.45 & 0.54 & 0.27 & 0.55 & 0.17 & 0.35 & 0.06 & 0.13 \\
\hline Caucasian American & 227 & 0.94 & 1.00 & 0.50 & 0.78 & 0.54 & 0.73 & 0.08 & 0.27 \\
\hline Multiracial & 8 & 1.04 & 0.96 & 0.43 & 0.64 & 0.87 & 0.76 & 0.14 & 0.39 \\
\hline \multicolumn{10}{|l|}{ Marital Status } \\
\hline Single, never married & 92 & 1.09 & 1.00 & 0.61 & 0.88 & 0.55 & 0.74 & 0.06 & 0.23 \\
\hline Engaged or Married & 155 & 0.87 & 1.00 & 0.48 & 0.75 & 0.53 & 0.72 & 0.10 & 0.32 \\
\hline Separated or Divorced & 26 & 1.13 & 0.95 & 0.81 & 0.91 & 0.77 & 0.86 & 0.07 & 0.25 \\
\hline Widowed & 3 & 1.44 & 1.36 & 0.00 & --- & 1.50 & 1.38 & 0.00 & --- \\
\hline \multicolumn{10}{|l|}{ Income } \\
\hline$\$ 0$ to $\$ 9,999$ & 7 & 1.77 & 0.91 & 0.90 & 0.90 & 0.41 & 0.73 & 0.10 & 0.26 \\
\hline$\$ 10,000$ to & 23 & 1.44 & 1.01 & 1.04 & 0.93 & 1.01 & 0.78 & 0.17 & 0.39 \\
\hline$\$ 25,000$ to $\$ 49,999$ & 64 & 0.91 & 0.98 & 0.40 & 0.68 & 0.49 & 0.68 & 0.05 & 0.20 \\
\hline$\$ 50,000$ to $\$ 74,999$ & 68 & 1.10 & 0.96 & 0.64 & 0.87 & 0.57 & 0.75 & 0.13 & 0.38 \\
\hline$\$ 75,000$ to $\$ 99,999$ & 53 & 0.74 & 1.00 & 0.35 & 0.73 & 0.57 & 0.77 & 0.03 & 0.18 \\
\hline$\$ 100,000$ to $\$ 124,999$ & 28 & 0.74 & 0.95 & 0.39 & 0.71 & 0.40 & 0.67 & 0.08 & 0.29 \\
\hline$\$ 125,000$ to $\$ 149,999$ & 12 & 0.88 & 1.11 & 0.56 & 0.72 & 0.77 & 1.00 & 0.06 & 0.20 \\
\hline$\$ 150,000$ to $\$ 174,999$ & 8 & 1.07 & 1.20 & 1.14 & 1.04 & 0.63 & 1.01 & 0.00 & --- \\
\hline$\$ 175,000$ to $\$ 199,999$ & 6 & 0.65 & 0.56 & 0.30 & 0.73 & 0.30 & 0.48 & 0.12 & 0.28 \\
\hline$\$ 200,000$ and up & 8 & 0.96 & 1.06 & 0.66 & 0.93 & 0.43 & 0.74 & 0.14 & 0.39 \\
\hline
\end{tabular}

*Two-week frequency values were natural log transformed 
Table 8-Continued

\begin{tabular}{|c|c|c|c|c|c|c|c|}
\hline \multirow[b]{2}{*}{ Characteristics } & \multirow[b]{2}{*}{$n$} & \multicolumn{2}{|c|}{ NSSI } & \multicolumn{2}{|c|}{ Ideation } & \multicolumn{2}{|c|}{ Attempts } \\
\hline & & $M$ & $S D$ & $M$ & $S D$ & $M$ & $S D$ \\
\hline \multicolumn{8}{|l|}{ Age } \\
\hline $18-29$ & 85 & 0.15 & 0.44 & 0.49 & 0.90 & 0.02 & 0.15 \\
\hline $30-39$ & 104 & 0.09 & 0.36 & 0.34 & 0.75 & 0.06 & 0.47 \\
\hline $40-49$ & 53 & 0.05 & 0.23 & 0.19 & 0.51 & 0.00 & --- \\
\hline $50-59$ & 25 & 0.03 & 0.14 & 0.08 & 0.30 & 0.00 & --- \\
\hline $60+$ & 10 & 0.00 & --- & 0.00 & --- & 0.00 & --- \\
\hline \multicolumn{8}{|l|}{ Sex } \\
\hline Female & 172 & 0.24 & 0.40 & 0.35 & 0.75 & 0.03 & 0.37 \\
\hline Male & 105 & 0.05 & 0.12 & 0.27 & 0.70 & 0.02 & 0.12 \\
\hline \multicolumn{8}{|l|}{ Ethnicity } \\
\hline African American & 16 & 0.28 & 0.62 & 0.42 & 0.67 & 0.09 & 0.35 \\
\hline Latino/a American & 18 & 0.06 & 0.26 & 0.16 & 0.38 & 0.00 & --- \\
\hline American Indian & 3 & 0.00 & --- & 1.14 & 1.98 & 0.00 & --- \\
\hline Asian American & 4 & 0.05 & 0.10 & 0.00 & --- & 0.00 & --- \\
\hline Caucasian American & 227 & 0.09 & 0.34 & 0.32 & 0.75 & 0.03 & 0.32 \\
\hline Multiracial & 8 & 0.00 & --- & 0.26 & 0.36 & 0.00 & --- \\
\hline \multicolumn{8}{|l|}{ Marital Status } \\
\hline Single, never married & 92 & 0.08 & 0.33 & 0.52 & 0.95 & 0.00 & --- \\
\hline Engaged or Married & 155 & 0.11 & 0.38 & 0.24 & 0.59 & 0.05 & 0.40 \\
\hline Separated or Divorced & 26 & 0.05 & 0.19 & 0.17 & 0.51 & 0.00 & --- \\
\hline Widowed & 3 & 0.00 & --- & 0.00 & --- & 0.00 & --- \\
\hline \multicolumn{8}{|l|}{ Income } \\
\hline$\$ 0$ to $\$ 9,999$ & 7 & 0.00 & --- & 0.80 & 1.38 & 0.00 & --- \\
\hline$\$ 10,000$ to $\$ 24,999$ & 23 & 0.27 & 0.58 & 0.73 & 1.01 & 0.00 & --- \\
\hline$\$ 25,000$ to $\$ 49,999$ & 64 & 0.78 & 0.27 & 0.45 & 0.82 & 0.01 & 0.09 \\
\hline$\$ 50,000$ to $\$ 74,999$ & 68 & 0.15 & 0.46 & 0.33 & 0.70 & 0.09 & 0.58 \\
\hline$\$ 75,000$ to $\$ 99,999$ & 53 & 0.01 & 0.10 & 0.14 & 0.51 & 0.01 & 0.10 \\
\hline$\$ 100,000$ to $\$ 124,999$ & 28 & 0.05 & 0.26 & 0.07 & 0.22 & 0.00 & --- \\
\hline$\$ 125,000$ to $\$ 149,999$ & 12 & 0.00 & --- & 0.49 & 1.07 & 0.00 & --- \\
\hline$\$ 150,000$ to $\$ 174,999$ & 8 & 0.00 & --- & 0.00 & --- & 0.00 & --- \\
\hline$\$ 175,000$ to $\$ 199,999$ & 6 & 0.27 & 0.66 & 0.00 & --- & 0.12 & 0.28 \\
\hline$\$ 200,000$ and up & 8 & 0.09 & 0.25 & 0.00 & --- & 0.00 & --- \\
\hline
\end{tabular}

*Two-week frequency values were natural log transformed 
Table 9

Lifetime Frequency of Self-Damaging Behaviors by Demographic Characteristics*

\begin{tabular}{|c|c|c|c|c|c|c|c|c|c|}
\hline \multirow[b]{2}{*}{ Characteristics } & \multirow[b]{2}{*}{$\mathrm{n}$} & \multicolumn{2}{|c|}{ Limiting } & \multicolumn{2}{|c|}{ Fasting } & \multicolumn{2}{|c|}{ Binging } & \multicolumn{2}{|c|}{ Purging } \\
\hline & & $M$ & $S D$ & $M$ & $S D$ & $M$ & $S D$ & $M$ & $S D$ \\
\hline \multicolumn{10}{|l|}{ Age } \\
\hline 18-29 & 85 & 3.78 & 2.30 & 2.45 & 2.34 & 2.47 & 2.42 & 1.05 & 1.84 \\
\hline $30-39$ & 104 & 3.34 & 2.83 & 2.76 & 2.55 & 3.04 & 2.49 & 1.17 & 1.75 \\
\hline $40-49$ & 53 & 4.59 & 2.89 & 3.29 & 2.85 & 3.47 & 2.38 & 1.26 & 1.76 \\
\hline $50-59$ & 25 & 4.19 & 3.28 & 2.96 & 2.65 & 3.33 & 2.82 & 1.04 & 2.20 \\
\hline $60+$ & 10 & 4.46 & 3.26 & 2.82 & 2.97 & 4.02 & 2.52 & 0.46 & 1.46 \\
\hline \multicolumn{10}{|l|}{ Sex } \\
\hline Female & 172 & 4.31 & 2.66 & 2.87 & 2.53 & 3.15 & 2.64 & 1.37 & 2.02 \\
\hline Male & 105 & 3.05 & 2.79 & 2.65 & 2.63 & 2.79 & 2.24 & 0.70 & 1.31 \\
\hline \multicolumn{10}{|l|}{ Ethnicity } \\
\hline African American & 16 & 4.09 & 2.14 & 3.55 & 2.14 & 2.88 & 2.33 & 1.00 & 1.31 \\
\hline Latino/a American & 18 & 2.71 & 2.70 & 1.85 & 2.46 & 2.64 & 2.35 & 1.50 & 2.33 \\
\hline American Indian & 3 & 4.50 & 1.85 & 4.27 & 1.97 & 3.97 & 4.54 & 0.00 & --- \\
\hline Asian American & 4 & 4.44 & 3.27 & 3.46 & 3.94 & 3.15 & 3.67 & 2.00 & 2.30 \\
\hline Caucasian American & 227 & 3.83 & 2.84 & 2.78 & 2.59 & 3.07 & 2.51 & 1.09 & 1.80 \\
\hline Multiracial & 8 & 4.85 & 1.58 & 2.22 & 1.84 & 1.95 & 1.94 & 0.88 & 1.73 \\
\hline \multicolumn{10}{|l|}{ Marital Status } \\
\hline Single, never married & 92 & 3.97 & 2.35 & 2.64 & 2.40 & 2.62 & 2.44 & 0.92 & 1.63 \\
\hline Engaged or Married & 155 & 3.64 & 2.96 & 2.65 & 2.56 & 3.11 & 2.49 & 1.20 & 1.91 \\
\hline Separated or Divorced & 26 & 4.43 & 3.01 & 4.17 & 2.89 & 3.91 & 2.61 & 1.43 & 1.84 \\
\hline Widowed & 3 & 5.58 & 0.85 & 3.15 & 3.20 & 3.35 & 2.02 & 0.00 & --- \\
\hline \multicolumn{10}{|l|}{ Income } \\
\hline$\$ 0$ to $\$ 9,999$ & 7 & 4.51 & 2.68 & 2.32 & 3.05 & 0.83 & 1.45 & 0.62 & 1.22 \\
\hline$\$ 10,000$ to $\$ 24,999$ & 23 & 3.96 & 2.89 & 3.17 & 2.79 & 3.61 & 2.47 & 1.54 & 2.41 \\
\hline$\$ 25,000$ to $\$ 49,999$ & 64 & 3.58 & 2.95 & 2.28 & 2.42 & 3.06 & 2.82 & 1.11 & 1.84 \\
\hline$\$ 50,000$ to $\$ 74,999$ & 68 & 3.98 & 2.55 & 3.23 & 2.56 & 2.92 & 2.30 & 1.22 & 1.68 \\
\hline$\$ 75,000$ to $\$ 99,999$ & 53 & 3.90 & 2.78 & 2.43 & 2.53 & 3.23 & 2.18 & 0.95 & 1.63 \\
\hline$\$ 100,000$ to $\$ 124,999$ & 28 & 4.00 & 2.78 & 2.51 & 2.44 & 2.75 & 2.51 & 1.03 & 1.74 \\
\hline$\$ 125,000$ to $\$ 149,999$ & 12 & 2.66 & 2.98 & 2.87 & 2.73 & 3.07 & 2.52 & 1.11 & 2.29 \\
\hline$\$ 150,000$ to $\$ 174,999$ & 8 & 3.34 & 3.44 & 4.97 & 3.03 & 4.69 & 3.34 & 0.88 & 1.73 \\
\hline$\$ 175,000$ to $\$ 199,999$ & 6 & 3.55 & 2.05 & 2.98 & 1.98 & 1.35 & 1.65 & 1.07 & 1.73 \\
\hline$\$ 200,000$ and up & 8 & 4.97 & 2.77 & 3.32 & 2.43 & 2.53 & 2.74 & 1.09 & 2.43 \\
\hline
\end{tabular}


Table 9-Continued

\begin{tabular}{|c|c|c|c|c|c|c|c|}
\hline \multirow{2}{*}{ Characteristics } & \multirow[b]{2}{*}{$n$} & \multicolumn{2}{|c|}{ NSSI } & \multicolumn{2}{|c|}{ Ideation } & \multicolumn{2}{|c|}{ Attempts } \\
\hline & & $M$ & $S D$ & $M$ & $S D$ & $M$ & $S D$ \\
\hline \multicolumn{8}{|l|}{ Age } \\
\hline $18-29$ & 85 & 1.60 & 1.77 & 2.22 & 2.34 & 0.25 & 0.48 \\
\hline $30-39$ & 104 & 1.46 & 1.73 & 2.21 & 2.26 & 0.34 & 0.60 \\
\hline $40-49$ & 53 & 0.78 & 1.22 & 1.65 & 1.88 & 0.36 & 0.59 \\
\hline $50-59$ & 25 & 0.37 & 1.04 & 1.65 & 1.45 & 0.20 & 0.37 \\
\hline $60+$ & 10 & 0.44 & 0.96 & 2.23 & 2.22 & 0.07 & 0.22 \\
\hline \multicolumn{8}{|l|}{ Sex } \\
\hline Female & 172 & 1.35 & 1.75 & 2.16 & 2.16 & 0.32 & 0.55 \\
\hline Male & 105 & 1.05 & 1.43 & 1.90 & 2.04 & 0.26 & 0.50 \\
\hline \multicolumn{8}{|l|}{ Ethnicity } \\
\hline African American & 16 & 0.69 & 1.09 & 1.31 & 1.05 & 0.57 & 0.79 \\
\hline Latino/a American & 18 & 0.90 & 1.22 & 1.63 & 1.76 & 0.29 & 0.39 \\
\hline American Indian & 3 & 0.54 & 0.93 & 3.85 & 4.06 & 0.00 & --- \\
\hline Asian American & 4 & 1.09 & 1.75 & 0.77 & 1.13 & 0.00 & --- \\
\hline Caucasian American & 227 & 1.33 & 1.70 & 2.14 & 2.16 & 0.29 & 0.53 \\
\hline Multiracial & 8 & 0.85 & 1.42 & 1.47 & 1.51 & 0.17 & 0.32 \\
\hline \multicolumn{8}{|l|}{ Marital Status } \\
\hline Single, never married & 92 & 1.46 & 1.77 & 2.35 & 2.22 & 0.31 & 0.54 \\
\hline Engaged or Married & 155 & 1.21 & 1.62 & 1.98 & 2.11 & 0.29 & 0.53 \\
\hline Separated or Divorced & 26 & 0.72 & 1.20 & 1.71 & 1.81 & 0.29 & 0.62 \\
\hline Widowed & 3 & 0.00 & --- & 0.60 & 1.03 & 0.23 & 0.40 \\
\hline \multicolumn{8}{|l|}{ Income } \\
\hline$\$ 0$ to $\$ 9,999$ & 7 & 2.15 & 2.75 & 3.25 & 3.94 & 0.93 & 1.00 \\
\hline$\$ 10,000$ to $\$ 24,999$ & 23 & 1.58 & 2.17 & 2.89 & 2.83 & 0.44 & 0.60 \\
\hline$\$ 25,000$ to $\$ 49,999$ & 64 & 1.67 & 1.78 & 2.45 & 1.96 & 0.33 & 0.58 \\
\hline$\$ 50,000$ to $\$ 74,999$ & 68 & 1.26 & 1.38 & 1.96 & 2.04 & 0.28 & 0.54 \\
\hline$\$ 75,000$ to $\$ 99,999$ & 53 & 0.88 & 1.36 & 1.39 & 1.62 & 0.18 & 0.41 \\
\hline$\$ 100,000$ to $\$ 124,999$ & 28 & 0.78 & 1.59 & 1.25 & 1.20 & 0.24 & 0.45 \\
\hline$\$ 125,000$ to $\$ 149,999$ & 12 & 0.99 & 1.45 & 3.49 & 3.43 & 0.38 & 0.51 \\
\hline$\$ 150,000$ to $\$ 174,999$ & 8 & 0.09 & 0.25 & 2.20 & 1.58 & 0.09 & 0.25 \\
\hline$\$ 175,000$ to $\$ 199,999$ & 6 & 1.69 & 1.55 & 2.37 & 1.18 & 0.38 & 0.66 \\
\hline$\$ 200,000$ and up & 8 & 0.97 & 1.60 & 1.30 & 1.31 & 0.17 & 0.32 \\
\hline
\end{tabular}

*Lifetime frequency values were natural log transformed 
Contrary to expectation, only some of the self-damaging behaviors were significantly correlated with DT. Two-week limiting, two-week fasting, lifetime purging, two-week purging, lifetime NSSI, two-week NSSI, lifetime ideation, two-week ideation, and lifetime attempts were correlated with DT. Likewise, only some of the self-damaging behaviors were correlated with anxiety and depression.

As anticipated, all of the two-week frequencies and lifetime frequencies of identical behaviors were correlated (ranging from .3 to .7) with each other. Beyond these relationships, the following behaviors were those most correlated within the selfdamaging behaviors:

1. Lifetime frequency of fasting was the behavior most highly correlated with lifetime frequency of limiting.

2. Two-week frequency of fasting was the behavior most highly correlated with two-week frequency of limiting.

3. Lifetime frequency of binging was the behavior most highly correlated with lifetime frequency of fasting.

4. Two-week frequency of purging was the behavior most highly correlated with two-week frequency of fasting.

5. Lifetime frequency of purging was the behavior most highly correlated with lifetime frequency of binging.

6. Two-week frequency of purging was the behavior most highly correlated with two-week frequency of binging. 
Table 10

Zero-Order Correlations

\begin{tabular}{lccccccccc}
\hline & $\begin{array}{c}\text { Emotional } \\
\text { Reactivity }\end{array}$ & $\begin{array}{c}\text { Learned } \\
\text { Help. }\end{array}$ & Anxiety & Depression & $\begin{array}{c}\text { Lifetime } \\
\text { limiting }\end{array}$ & $\begin{array}{c}\text { 2-week } \\
\text { limiting }\end{array}$ & $\begin{array}{c}\text { Lifetime } \\
\text { fasting }\end{array}$ & $\begin{array}{c}2 \text {-week } \\
\text { fasting }\end{array}$ & $\begin{array}{c}\text { Lifetime } \\
\text { binging }\end{array}$ \\
\hline Dist. Tol. & $-.686^{* *}$ & $-.539^{* *}$ & $-.598^{* *}$ & $-.541^{* *}$ & -.062 & $-.147^{*}$ & -.103 & $-.166^{* *}$ & -.013 \\
Emot. React. & & $.416^{* *}$ & $.549^{* *}$ & $.527^{* *}$ & .037 & .116 & .042 & .110 & .049 \\
Learn. Help. & & & $.521^{* *}$ & $.568^{* *}$ & .113 & $.130^{*}$ & .072 & $.122^{*}$ & .029 \\
Anxiety & & & & $.672^{* *}$ & .025 & $.153^{*}$ & .062 & $.209^{* *}$ & .041 \\
Depression & & & & & .096 & $.159^{* *}$ & $.155^{* *}$ & $.295^{* *}$ & $.124^{*}$ \\
L limiting & & & & & & $.667^{* *}$ & $.578^{* *}$ & $.359^{* *}$ & $.287^{* *}$ \\
2 limiting & & & & & & & $.399^{*}$ & $.578^{* *}$ & $.215^{* *}$ \\
L fasting & & & & & & & $.634^{* *}$ & $.311^{* *}$ \\
2 fasting & & & & & & & & $.244^{* *}$ \\
\hline
\end{tabular}

근 
Table 10-Continued

\begin{tabular}{|c|c|c|c|c|c|c|c|c|c|}
\hline & $\begin{array}{l}2 \text {-week } \\
\text { binging }\end{array}$ & $\begin{array}{l}\text { Lifetime } \\
\text { purging }\end{array}$ & $\begin{array}{l}2 \text {-week } \\
\text { purging }\end{array}$ & $\begin{array}{l}\text { Lifetime } \\
\text { NSSI }\end{array}$ & $\begin{array}{c}\text { 2-week } \\
\text { NSSI }\end{array}$ & $\begin{array}{l}\text { Lifetime } \\
\text { ideation }\end{array}$ & $\begin{array}{c}2 \text {-week } \\
\text { ideation }\end{array}$ & $\begin{array}{l}\text { Lifetime } \\
\text { attempts }\end{array}$ & $\begin{array}{c}\text { 2-week } \\
\text { attempts }\end{array}$ \\
\hline Dist. Tol. & -.076 & $-.121 *$ & $-.157 * *$ & $-.358 * *$ & $-.158 * *$ & $-.339 * *$ & $-.289 * *$ & $-.231 * *$ & -.028 \\
\hline Emot. React. & .107 & $.151 *$ & $.174 * *$ & $.302 * *$ & $.167 * *$ & $.284 * *$ & $.222 * *$ & $.228 * *$ & .019 \\
\hline Learn. Help. & $.163 * *$ & .051 & .086 & $.188 * *$ & .106 & $.175^{* *}$ & $.216^{* *}$ & $.149 *$ & .053 \\
\hline Anxiety & $.163 * *$ & $.162 * *$ & $.273 * *$ & $.342 * *$ & $.317 * *$ & $.230 * *$ & $.249 * *$ & $.266^{* *}$ & .097 \\
\hline Depression & $.211 * *$ & $.139 *$ & $.246^{* *}$ & $.341 * *$ & $.272 * *$ & $.429 * *$ & $.434 * *$ & $.296^{* *}$ & .036 \\
\hline L limiting & .070 & $.370 * *$ & $.199 * *$ & .074 & .084 & .095 & .073 & -.021 & .021 \\
\hline 2 limiting & $.225 * *$ & $.318 * *$ & $.294 * *$ & .053 & $.191 * *$ & .011 & .109 & .052 & .075 \\
\hline L fasting & .050 & $.297 * *$ & $.190 * *$ & .113 & $.127 *$ & $.215^{* *} *$ & .116 & .023 & .032 \\
\hline 2 fasting & $.208 * *$ & $.265^{* *}$ & $.337 * *$ & $.161 * *$ & $.296 * *$ & $.184 * *$ & $.232 * *$ & $.132 *$ & $.149 *$ \\
\hline $\mathrm{L}$ binging & $.605 * *$ & $.301 * *$ & $.151^{* *}$ & .021 & .036 & .069 & -.016 & -.073 & .012 \\
\hline 2 binging & & .050 & $.283 * *$ & -.017 & $.189 * *$ & .019 & .116 & .077 & $.146^{*}$ \\
\hline L purging & & & $.585^{* *}$ & $.235 * *$ & $.290 * *$ & $.123 *$ & .031 & $.137 *$ & $.139 *$ \\
\hline 2 purging & & & & $.318 * *$ & $.571 * *$ & $.136^{*}$ & $.193 * *$ & $.246^{* *}$ & $.334 * *$ \\
\hline L NSSI & & & & & $.474 * *$ & $.510 * *$ & $.383 * *$ & $.287 * *$ & $.138 *$ \\
\hline 2 NSSI & & & & & & $.188 * *$ & $.212 * *$ & $.234 * *$ & $.439 * *$ \\
\hline L ideation & & & & & & & $.714 * *$ & $.409 * *$ & .070 \\
\hline 2 ideation & & & & & & & & $.371 * *$ & $.196 * *$ \\
\hline L attempts & & & & & & & & & $.304 * *$ \\
\hline
\end{tabular}


7. Lifetime frequency of binging was the behavior most highly correlated with lifetime frequency of purging.

8. Two-week frequency of NSSI was the behavior most highly correlated with two-week frequency of purging.

9. Lifetime frequency of suicidal ideation was the behavior most highly correlated with lifetime frequency of NSSI.

10. Two-week frequency of purging was the behavior most highly correlated with two-week frequency of NSSI.

11. Lifetime frequency of NSSI was the behavior most highly correlated with lifetime frequency of suicidal ideation.

12. Lifetime frequency of suicide attempts was the behavior most highly correlated with two-week frequency of suicidal ideation.

13. Lifetime suicidal ideation was the behavior most highly correlated with lifetime frequency of suicide attempts.

14. Lifetime frequency of NSSI was the behavior most highly correlated with two-week frequency of suicide attempts.

\section{Hypotheses Testing}

The first hypothesis of this study was that the reproduced covariance matrices $\Sigma(\gamma)$ implied in the two-week frequency theoretical model (Figure 1) and the observed sample covariance matrices $S$ were equal. The second hypothesis of this study was that the reproduced covariance matrices $\Sigma(\gamma)$ implied in the lifetime frequency theoretical model (Figure 2) and the observed sample covariance matrices $S$ were equal. For both models, the null hypothesis was rejected. As such, 
the models were respecified based on theory and modification indices. These modifications will be detailed below. The fit indices for the original and fitted models are presented in Table 11.

\section{Two-Week Frequency Model}

\section{Original Two-Week Model}

The two-week frequency model was a poor fit for the data. Chisquare for the original model was $1238.102(d f=558)$ with a probability level of $<.001$. This was not surprising given the large sample size of the study and the skewness of some of the data, which can both prevent the Chi-square from reaching non-significance at the .05 level. Therefore, as anticipated, the model was evaluated on the basis of fit indices. The criteria used to determine acceptable model fit was: Goodness of Fit Index (GFI $\geq .90)$, Normed Fit Index (NFI $\geq .95$ ), Comparative Fit Index (CFI $\geq .95)$, and Root Mean Square Error of Approximation (RMSEA; $\leq .06$; Cut-off values were determined according to the recommendations of Hooper et al., 2008). The fit indices for the hypothesized model were as follows: $\mathrm{GFI}=.80, \mathrm{NFI}=.77, \mathrm{CFI}=.86, \mathrm{RMSEA}=.06(.06$ to $.07)$.

\section{Fitted Two-Week Model}

Modification indices were examined in order to determine how the model could be revised to be more accurate. The final model is depicted in Figure 3. First, Depression and anxiety did not remain significant as controls and were removed. 
Table 11

Chi-Square and Goodness of Fit Indices for Hypothesized and Respecified Models

\begin{tabular}{|c|c|c|c|c|c|c|c|}
\hline Model & $\mathbf{X}^{2}$ & $p$ & $d f$ & GFI & NFI & CFI & RMSEA \\
\hline \multicolumn{8}{|c|}{ Two-week Frequency } \\
\hline Original & 1238.10 & $<.001$ & 588 & .80 & .77 & .86 & $\begin{array}{l}.06 \\
(.06 \text { to } .07)\end{array}$ \\
\hline Respecified & 275.62 & $<.001$ & 200 & .92 & .90 & .97 & $\begin{array}{l}.04 \\
(.03 \text { to } .05)\end{array}$ \\
\hline \multicolumn{8}{|c|}{ Lifetime Frequency } \\
\hline Original & 1306.54 & $<.001$ & 588 & .79 & .76 & .85 & $\begin{array}{l}.07 \\
(.06 \text { to } .07)\end{array}$ \\
\hline Respecified & 455.69 & $<.001$ & 312 & .89 & .89 & .96 & $\begin{array}{l}.04 \\
(.03 \text { to } .05)\end{array}$ \\
\hline
\end{tabular}

Note. $\mathrm{X}^{2}=$ Chi square test; $d f=$ degrees of freedom; GFI=Goodness of Fit Index; NFI=Normed Fit index; CFI=Comparative Fit Index; RMSEA=Root Mean Square Error of Approximation 


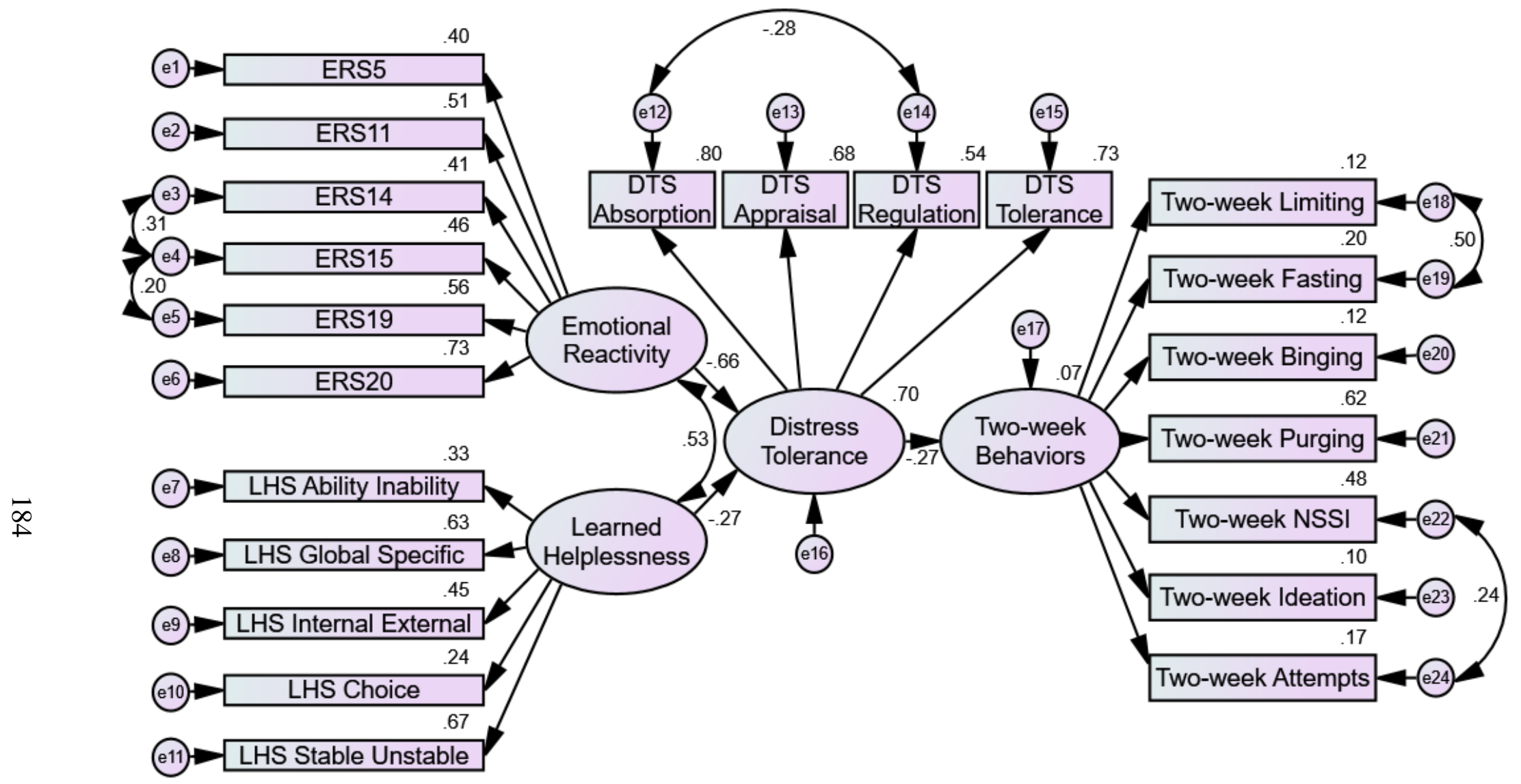

Figure 3. Fitted Two-Week Model 
Second, suggested correlations which are theoretically consistent were added. A significant correlation between emotional reactivity and learned helplessness was added $(r=.53)$. Correlations were also added between the error terms on item 14 and item 15 ( $r$ $=.31)$ and between the error terms on item 15 and $19(r=.20)$ on the ERS. A correlation was added between the error terms on DTS Absorption and DTS Regulation $(r=-.28)$. Finally, correlations were added between the error terms on two-week limiting and twoweek fasting $(r=.50)$ and between the error terms on two-week NSSI and two-week suicide attempts $(r=.24)$.

The resulting model was well fit to the data. As was suspected, the Chi-square for the fitted model still failed to reach non-significance (Chi-square $=275.62, d f=200, p<$ .001) because of the large sample size and skewness of some of the data. Given the strength of other values, NFI was considered acceptable at .90. GFI indicated good fit at .92. CFI indicated a strong fit at .97. RMSEA also indicated strong fit at .04 (.03 to .05).

\section{Intercorrelations Among Variables}

Table 12 shows the causal effects of the revised two-week model. In the revised model, the combination of emotional reactivity and learned helplessness accounted for $70 \%$ of the variance in DT. Emotional reactivity was the strongest predictor of DT ( $\beta$ $=-.66, p<.001)$. Overall, the model accounted for $7 \%$ of the variance in two-week frequency of self-damaging behaviors. Distress tolerance was directly related to twoweek frequency of self-damaging behaviors. Both emotional reactivity and learned helplessness had significant indirect positive effects on the frequency of self-damaging behaviors, such that higher emotional reactivity and higher learned helplessness were significantly correlated with higher frequency of self-damaging behaviors. 
Table 12

Summary of Causal Effects of Fitted Two-Week Model

\begin{tabular}{lllcc}
\hline \multirow{2}{*}{ Outcome } & \multicolumn{1}{c}{ Determinant } & \multicolumn{3}{c}{ Causal Effects } \\
\cline { 3 - 5 } Distress & Emotional Reactivity** & -.66 & --- & -.66 \\
Tolerance & Learned Helplessness** & -.27 & --- & -.27 \\
$\left(R^{2}=.70\right)$ & & & & \\
& & & & \\
Self-damaging & Emotional Reactivity** & --- & .18 & .18 \\
behaviors & Learned Helplessness** & --- & .07 & .07 \\
$\left(R^{2}=.07\right)$ & Distress Tolerance** & -.27 & --- & -.27 \\
\hline$* * p<.01$. & & & &
\end{tabular}

Table 13 shows the standardized coefficients in the revised two-week model.

Emotional reactivity's strong direct effect was $\beta=-.66(p<.001)$ and learned helplessness' strong direct effect was $\beta=-.27$ ( $p<.001)$. Thus, higher emotional reactivity and higher learned helplessness were significantly correlated with lower DT. The strong direct effect of DT on self-damaging behaviors was $\beta=-.27(p=.001)$, indicating that higher DT is significantly correlated with lower frequency of selfdamaging behaviors.

Table 13

Raw and Standardized Coefficients for the Revised Two-Week Model

\begin{tabular}{lcccc}
\hline Paths & $\mathrm{b}$ & $\mathrm{SE}$ & $\beta$ & $\mathrm{p}$ \\
\hline Emotional reactivity $\longrightarrow$ Distress tolerance & -.58 & .06 & -.66 & $<.001$ \\
Learned helplessness $\rightarrow$ Distress tolerance & -.12 & .02 & -.27 & $<.001$ \\
Distress tolerance $\longrightarrow$ Self-damaging behaviors & -.04 & .01 & -.27 & .001 \\
\hline
\end{tabular}




\section{Lifetime Frequency Model}

\section{Original Lifetime Model}

The lifetime frequency model was a poor fit for the data. Chi-square for the original model was $1306.525(d f=558)$ with a probability level of $<.001$. This was not surprising given the large sample size of the study and the skewness of some of the data, which can both prevent the Chi-square from reaching non-significance at the .05 level. Therefore, as anticipated, the model was evaluated on the basis of fit indices. The fit indices for the hypothesized model were as follows: $\mathrm{GFI}=.79, \mathrm{NFI}=.76, \mathrm{CFI}=.85$, RMSEA $=.07$ (.06 to .07$)$.

\section{Fitted Lifetime Model}

Modification indices were examined in order to determine how the model could be revised to be more accurate. The final lifetime model is depicted in Figure 4. First, age and anxiety did not remain significant as controls and were removed. Depression remained significant and was retained as a control. Depression was also observed to be correlated with both emotional reactivity and learned helplessness.

Second, suggested correlations which are theoretically consistent were added. A significant correlation between emotional reactivity and learned helplessness was added $(r=.53)$. Correlations were also added between the error terms on item 14 and item 15 ( $r$ $=.31)$ and between the error terms on item 15 and $19(r=.20)$ on the ERS. A correlation was added between the error terms on DTS Absorption and DTS Regulation $(r=-.29)$. A final correlation was added between the error terms on lifetime fasting and lifetime purging $(r=.26)$. 


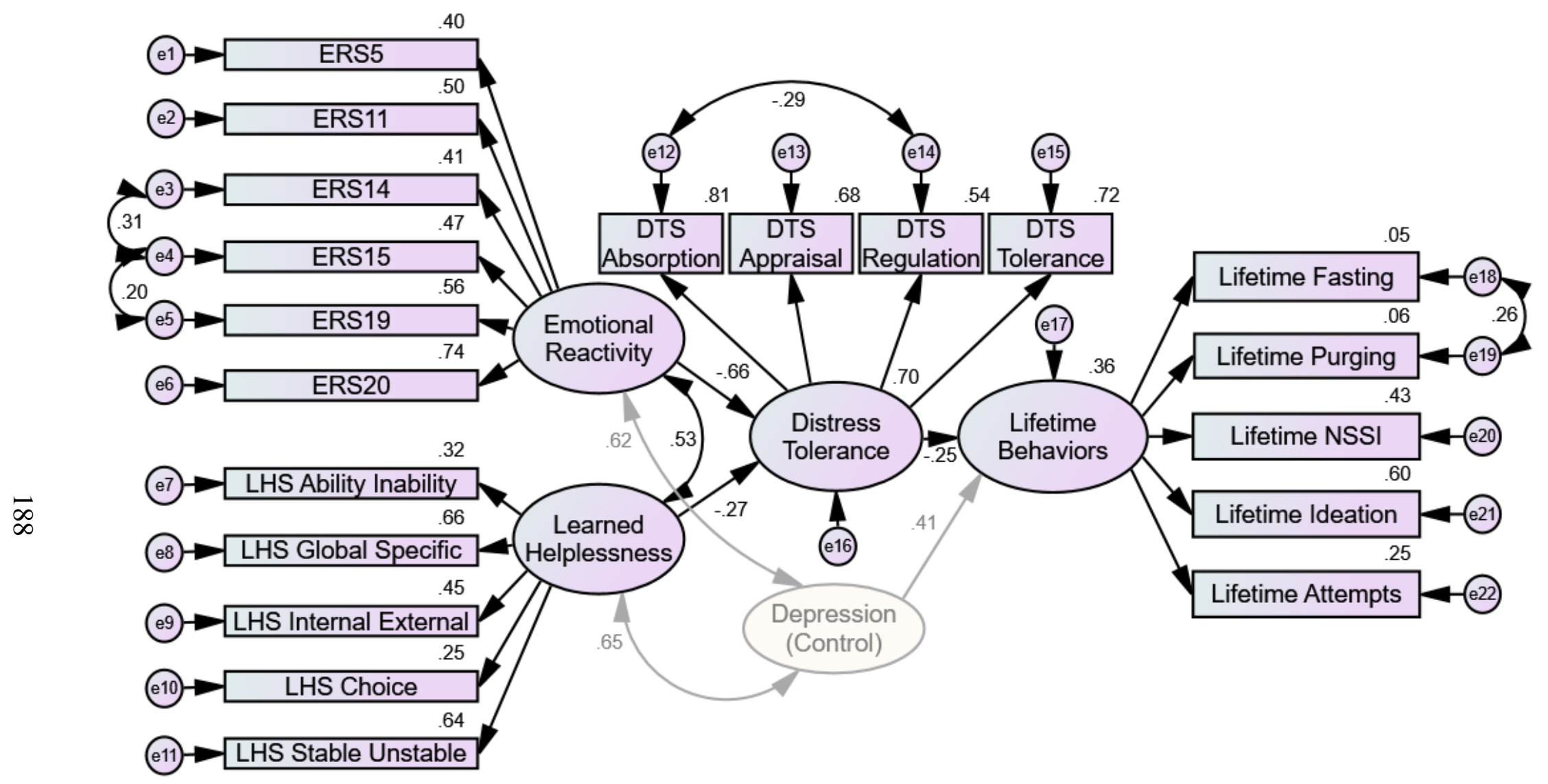

Figure 4. Fitted Lifetime Model 
Lastly, as intercorrelations among the error terms of the self-damaging behaviors were added, two of the items from the Self-damaging Behaviors Questionnaire did not retain their loading on the latent variable representing lifetime frequency of selfdamaging behaviors. So, these items (lifetime limiting and lifetime binging) were removed. The following items were retained: lifetime fasting, lifetime purging, lifetime NSSI, lifetime ideation, lifetime attempts.

The resulting model was well fit to the data. As was anticipated, the Chi-square for the fitted model still failed to reach non-significance (Chi-square $=455.69, d f=312$, $p<.001)$ because of the large sample size and skewness of some of the data. Given the strength of other values, NFI was considered acceptable at .89 and GFI was considered acceptable at .89 . CFI indicated a strong fit at .96. RMSEA also indicated strong fit at .04 (.03 to .05$)$.

\section{Intercorrelations Among Variables}

Table 14 shows the causal effects of the revised lifetime model. In the revised model, the combination of emotional reactivity and learned helplessness accounted for $70 \%$ of the variance in DT. Emotional reactivity was the strongest predictor of DT ( $\beta$ $=-.66)$. Overall, the model accounted for $36 \%$ of the variance in lifetime frequency of self-damaging behaviors. Depression and DT were the strongest predictors of selfdamaging behaviors. Both emotional reactivity and learned helplessness had indirect positive effects on the frequency of self-damaging behaviors, such that higher emotional reactivity and higher learned helplessness were significantly correlated with higher frequency of self-damaging behaviors. 
Table 14

Summary of Causal Effects of Fitted Lifetime Model

\begin{tabular}{llccc}
\hline \multirow{2}{*}{ Outcome } & \multicolumn{1}{c}{ Determinant } & \multicolumn{3}{c}{ Causal Effects } \\
\cline { 3 - 5 } $\begin{array}{l}\text { Distress } \\
\text { Tolerance }\end{array}$ & Emotional Reactivity** & -.66 & --- & -.66 \\
$\left(R^{2}=.70\right)$ & Learned Helplessness** & -.27 & --- & -.27 \\
& & & & \\
Self-damaging & Emotional Reactivity** & --- & .17 & .17 \\
behaviors & Learned Helplessness** & --- & .07 & .07 \\
$\left(R^{2}=.36\right)$ & Depression** & .41 & --- & .41 \\
& Distress Tolerance** & -.25 & --- & -.25 \\
\hline$* * p<.01$. & & & &
\end{tabular}

Table 15 shows the standardized coefficients in the revised lifetime model.

Emotional reactivity's strong direct effect was $\beta=-.66(p<.001)$ and learned

helplessness' strong direct effect was $\beta=-.27$ ( $p<.001)$. Thus, higher emotional

reactivity and higher learned helplessness were significantly correlated with lower DT.

The moderate direct effect of DT on self-damaging behaviors was $\beta=-.25(p=.004)$,

indicating that higher DT is significantly correlated with lower frequency of self-

damaging behaviors. The strong direct effect of depression on self-damaging behaviors

was $\beta=.41(p<.001)$, indicating that higher depression was correlated with higher

frequency of self-damaging behaviors.

\section{Summary}

The results of this study were described in this chapter. First, characteristics of the sample including data screening steps and instrument reliability were described. Then, characteristics of the variables were described. Lastly, results of SEM analyses were 
shown and described, including steps taken to respecify the model. Chapter 5 will discuss the implications of the results in light of the research questions and extant literature.

Table 15

Raw and Standardized Coefficients for the Revised Lifetime Model

\begin{tabular}{lcccc}
\hline Paths & $b$ & $S E$ & $\beta$ & $p$ \\
\hline Emotional reactivity $\longrightarrow$ Distress tolerance & -.58 & .06 & -.66 & $<.001$ \\
Learned helplessness $\longrightarrow$ Distress tolerance & -.12 & .03 & -.27 & $<.001$ \\
Distress tolerance $\rightarrow$ Self-damaging behaviors & -.07 & .03 & -.25 & .004 \\
Depression $\longrightarrow$ Self-damaging behaviors & .14 & .03 & .41 & $<.001$ \\
\hline
\end{tabular}




\section{CHAPTER 5}

\section{SUMMARY, DISCUSSION, AND IMPLICATIONS}

\section{Introduction}

In this chapter, I will first summarize the content of the previous four chapters. The purpose of the study will be outlined, the existing literature will be summarized, the methodology will be described, and the results will be presented. Then, I will discuss the findings in relation to the existing literature. I will identify limitations to the current study, implications for future practice, and implications for future research.

\section{Purpose of the Study}

The first purpose of this study was to review current literature about DT, individual difference factors in DT, and the relationship between DT and self-damaging behaviors. This purpose was achieved through the thorough literature review, which will be summarized below. The second purpose of this study was to test two models of individual difference factors in DT in the context of self-damaging behaviors (restricting, fasting, binging, purging, NSSI, suicidal ideation, and suicide attempts). In particular, I sought to investigate the role of two potential individual difference factors in DTemotional reactivity and learned helplessness. The first model examined this relationship in the context of two-week frequency of self-damaging behaviors, and the second model examined this relationship in the context of lifetime frequency of self-damaging 
behaviors. The fit of the models was described in the previous chapter and the implications of the results will be described below. The findings from this study contribute to the literature on DT and to the knowledge of the relationship between DT and self-damaging behaviors.

\section{Summary of the Literature Review}

Emotional distress tolerance is defined as one's perceived ability to withstand negative emotional states (Simons \& Gaher, 2005; Zvolensky et al., 2011). Distress tolerance is a concept with importance across multiple diagnostic categories (Bardeen et al., 2013; Kiselica et al., 2014). In particular, low DT has been linked to behaviors that often immediately reduce the individual's distress, but which cause psychological and often physical harm by avoiding real-life issues or problems (McHugh et al., 2013). Distress tolerance is represented by a single higher-order factor with four lower-order factors: absorption, appraisal, regulation, and tolerance (Simons \& Gaher, 2005). Distress tolerance is quite stable across time, unless targeted attempts to change DT level are made through clinical intervention (Kiselica et al., 2014; Macatee et al., 2015; McHugh et al., 2014; Simons \& Gaher, 2005). Initial evidence suggests that targeted interventions drawn from CBT (McHugh et al., 2014; Williams et al., 2013) and DBT (Booth et al., 2014) as well as DT-specific interventions (Bornovalova et al., 2012) may increase DT level.

Although DT is impacted by a number of factors (e.g., emotional regulation, attentional control, mindfulness, personality traits, parenting, and biology), unexplained individual differences in DT remain (G. Feldman et al., 2014). This lack of knowledge has hindered the development of prevention programs and clinical interventions. Drawing 
from the biosocial theory of emotional dysregulation developed by Linehan (2015) and the model of individual difference factors in DT developed by Lynch and Mizon (2011), emotional reactivity and learned helplessness were identified as potential individual difference factors in DT to be investigated in this study.

As demonstrated by Nock et al. (2008), emotional reactivity appears to be a single factor that is related to several serious psychological disorders (e.g., mood, anxiety, and eating disorders) and to serious behavioral symptoms (e.g., NSSI and suicidal ideation). Additionally, emotional reactivity appears likely to be a contributing individual difference factor in DT and appears to relate to a wide variety of negative behaviors including borderline personality symptoms (Bornovalova et al., 2011), depression symptoms (Ellis et al., 2013), heavy drinking (Winward et al., 2014), NSSI (Nock \& Mendes, 2008), and various anxiety symptoms (Cougle et al., 2013; Macatee et al., 2015; Marshall et al., 2008) in a similar way as DT. There are very few studies (Macatee \& Cougle, 2013; Zvolensky et al., 2011) that suggest that emotional reactivity and DT relate differently to negative behaviors (e.g., negative response to emotional films, and smoking). The studies that do exist seem methodologically weak, especially as compared to the number of studies supporting emotional reactivity as an individual difference factor. Therefore, this study examined emotional reactivity as an individual difference factor in DT in relation to self-damaging behaviors.

Learned helplessness is a factor conceptualized as involving low or no belief in personal agency (Seligman, 1975, as cited in Eisenberger et al., 1976). High learned helplessness interferes with an individual's motivation to work out a solution that terminates unfavorable conditions and to seek positive rewards (both of which may 
contribute to harmful behaviors and psychological disorders). There are no known studies that directly examined learned helplessness in relation to DT. Results regarding the relationship between learned helplessness and physical pain tolerance are mixed $(\mathrm{H}$. Feldman, 1986). However, high helplessness occurred together with low DT in a sample of individuals with NSSI (Slee et al., 2008). Additionally, learned helplessness has been theoretically linked to an individual opting for actions with immediate negative reinforcement (e.g., NSSI) despite long-term harm, rather than opting for actions which require high-effort and time before negative reinforcement occurs (e.g., distress tolerance skills; Trafton \& Gifford, 2011). Therefore, this study explored learned helplessness as an individual difference factor in DT in relation to self-damaging behaviors.

Research repeatedly, although not invariably, shows DT to be correlated with both anxiety and depression (Norr et al., 2014). DT has also been shown to have a predictive role in various anxiety symptoms including AS (Johnson et al., 2012), health anxiety (Fergus et al., 2015), panic symptoms (Keough et al., 2010), and worry (Keough et al., 2010). Yet, anxiety and depression are typically separate factors from DT. Since anxiety and depression may relate to outcome variables in a similar manner as DT, this study included both anxiety and depression as controls.

Distress tolerance is thought to have transdiagnostic relevance, and has been studied in relation to many different diagnostic and clinical concerns (for a review see Leyro, 2010). The study of DT is particularly important because the existing literature supports a relationship between DT and self-damaging behaviors (Anestis, Pennings, et al., 2013; Anestis et al., 2007). Distress tolerance is negatively correlated with unhealthy eating behaviors including body disordered eating patterns (Kelly et al., 2014), bulimic 
symptoms (Anestis et al., 2007; Corstorphine et al., 2007; Lavender et al., 2015), body dissatisfaction (Anestis et al., 2007; Corstorphine et al., 2007; Lavender et al., 2015), disinhibited eating (Lydecker et al., 2014), desire to be thinner (Anestis et al., 2007; Lydecker et al., 2014), and binge eating symptoms (Kenardy et al., 1996). Distress tolerance is also negatively correlated with both NSSI lifetime frequency and number of NSSI methods (Anestis, Kleiman, et al., 2014; Anestis, Knorr, et al., 2013; Anestis, Pennings, et al., 2013; Peterson et al., 2014). An intervention aimed at increasing DT skills successfully decreased NSSI frequency (Booth et al., 2014). Additionally, selfreported DT has been shown to be negatively correlated with suicidal desire (Anestis, Bagge, et al., 2011; Anestis, Moberg, et al., 2014), suicide potential (Anestis, Knorr, et al., 2013), and lifetime suicide attempts (Anestis, Kleiman, et al., 2014; Anestis, Pennings, et al., 2013). This pattern contrasts with a positive correlation observed between self-reported DT and acquired capability for suicide (Anestis, Bagge, et al., 2011). Overall, there is strong evidence for the role of DT in these severe, self-damaging behaviors. So, a more comprehensive model for individual difference factors in DT was needed in order to better inform the development of prevention and intervention protocols to reduce these risks (Marshall et al., 2008).

\section{Methodology}

This study employed a nonexperimental research design using self-report surveys. The sample consisted of adults, 18 years or older, who resided in the United States of America, and who had experienced at least one of the self-harming behaviors of interest. Participants were individuals who signed up to complete surveys through QuestionPro, an online research platform. 
All variables were quantified by participant self-report. Participants completed surveys which measured their (a) emotional reactivity, (b) learned helplessness, (c) DT, (d) depression, (e) anxiety, (f) two-week frequency of self-damaging behaviors, and (g) lifetime frequency of self-damaging behaviors. Emotional reactivity was measured with the ERS. Learned helplessness was measured with the LHS. Distress tolerance was measured with the sDTS. Two-week and lifetime frequency of self-damaging behavior were measured with the Self-damaging Behavior Questionnaire which was compiled for this study from the EDE-Q and the SITBI. Structural equation modeling was used to test two models for the role of emotional reactivity and learned helplessness in DT. One model was in the context of two-week frequency of self-damaging behaviors and the other model was in the context of lifetime frequency of self-damaging behaviors.

\section{Findings and Discussion}

\section{Respondent's Demographic Characteristics}

Respondents were drawn from a nonclinical sample, recruited through QuestionPro. A total of 694 individuals clicked on the link to my survey. However, 417 cases were excluded from data analysis due to not starting the survey, not completing the survey, not meeting inclusion criteria, or through the data screening process. The final sample consisted of 277 adults who completed the full survey, were at least the age of 18 , and endorsed a lifetime history of at least one self-damaging behavior. The sample was $62 \%$ female, $82 \%$ Caucasian, and $56 \%$ engaged or married. Respondents ranged in age from 18-79, with a mean age of 36.

The broad distribution of age in my study contributes to the existing literature since most former studies of DT with nonclinical populations have been conducted with 
college students. While the majority of respondents were Caucasian (82\%), the representation of minority ethnicities (18\%) exceeded representation of minority ethnicities in the general online population (13\%). The representation of marital status and income levels was also sufficiently broad. However, representation of males was less (38\%) than anticipated compared to the general online population (47\%).

Given that the survey link was initially distributed to a representative sample through QuestionPro, the fact that the majority of the retained respondents were females likely speaks to the true incidence of self-damaging behaviors among sexes. Thus, my results are relevant to the individuals most likely to be in need of clinical services related to self-damaging behaviors. However, future research will be needed to assess if the results of this study also apply broadly to males and other demographic groups. Nevertheless, the majority of the existing research used even less representative samples given that studies were typically conducted either with university students as a nonclinical population or with clinical samples.

\section{Incidence of Self-Damaging Behaviors}

One of the challenges in planning this study was anticipating how many respondents would endorse a lifetime history of one of the self-damaging behaviors (fasting, restricting, binging, purging, NSSI, suicidal ideation, and suicide attempts). This information was needed to calculate how many people the survey would need to be distributed to in order to achieve the desired sample size. Our knowledge based on the current literature was limited to the additive incidence rates of the individual behaviors. Results of this study revealed that in an online, non-clincical adult sample about twothirds $(61 \%)$ endorsed a lifetime history of at least one of the self-damaging behaviors. 
First, this finding is important because it may help future researchers plan for survey distribution. Second, this finding is important for clinicians as it emphasizes the prominence of self-damaging behaviors in nonclinical populations. Given the prominence in the general public, screening for these behaviors upon an individual's entry into clinical treatment seems crucial in developing informed treatment targets and failing to assess for self-damaging behaviors may miss vital clinical data present for most clients. This is particularly true since individuals who engage in self-damaging behaviors may not voluntarily disclose about the behaviors to clinicians. For example, Whitlock et al. (2011) found that only $17 \%$ of university students who endorsed NSSI and had attended therapy reported disclosing the NSSI to the mental health professional. Third, this finding should inform the development of health-promoting programming. When combined with the knowledge that individuals engaging in self-damaging behaviors may not seek clinical care (e.g., NSSI, American Psychiatric Association, 2013; Evans et al., 2005), this research suggests that self-damaging behaviors might be important targets of nonclincal prevention and reduction initiatives (e.g. in schools, in medical centers, through social media).

\section{Variable Description by Demographic Characteristics}

While examining group differences was outside the scope of this study, a few potential trends were identified across variables by demographic group. First, younger participants appeared to report higher two-week and lifetime frequencies of NSSI than older participants. Futher research is needed to determine if this difference exists, and if so, if this is due to an age effect or to a cohort effect. Second, females appeared to have higher mean scores on learned helplessness and emotional reactivity and lower mean 
scores on DT than males. Females also appeared to have higher mean scores on all twoweek and lifetime frequencies of self-damaging behaviors, except for two-week frequency of purging. Further research is needed to determine if this difference by sex truly exists. If so, it should be noted that a difference may be representative of a sociocultural effect rather than lower emotional health. For example, it may be that women endorse the self-damaging behaviors studied here at higher frequencies than men, but would endorse different self-damaging behaviors not observed in this study at lower frequencies than men.

\section{Zero-Order Correlations}

The correlations between emotional reactivity, learned helplessness, and DT were in the moderate range in this study. This supports that these variables should continue to be studied as distinct constructs. The significant correlations which existed between variables were in the theoretically expected directions, indicating that the variables performed typically in my sample.

However, contrary to expectation, some of the self-damaging behaviors were not correlated with DT. Specifically, lifetime limiting, lifetime fasting, two-week binging, lifetime binging, and two-week suicide attempts were not correlated with DT. This may have been due to low endorsement of some of the individual behaviors (e.g., two-week suicide attempts) or the skewness and kurtosis of some of the two-week self-damaging behaviors. Or, it may reflect a truly limited relationship between DT and these selfdamaging behaviors. So, futher research is needed to clarify the role, if any, of DT in these self-damaging behaviors. 
Between the self-damaging behaviors, there were a few correlations of note: lifetime limiting with lifetime fasting $(r=.578)$, two-week limiting with two-week fasting $(r=.578)$, two-week purging with two-week NSSI $(r=.571)$, lifetime suicidal ideation with lifetime NSSI $(r=.510)$, two-week NSSI with two-week suicide attempts $(r$ $=.439)$, and lifetime ideation with lifetime suicide attempts $(r=.409)$. Given these correlations, it is recommended that clinicians screen for the co-morbidity of these behaviors. It is also recommended that future researchers consider including these correlated self-damaging behaviors when studying an individual self-damaging behavior.

\section{Two-Week Model Fit}

Structural equation modeling was used to evaluate whether the hypothesized relationships among variables in the two-week frequency model were supported in this sample. Structural equation modeling indicated that the original model was a poor fit for the data, so revisions were made on the basis of the modification indices and theory.

First, depression and anxiety did not remain significant as controls and were removed. Thus, in this nonclinical sample, levels of depression and anxiety did not explain variance in two-week frequency of self-damaging behaviors. The extant literature suggests a relationship between anxiety and depression and the self-damaging behaviors. So, further research is needed to identify why this relationship was not significant for this sample. One possibility is that depression and anxiety may be related to the presence of the behaviors, rather than the severity (frequency) of the behaviors. Another possibility is that the limited possible range of self-damaging behavior frequency over a two-week period may not have been broad enough to capture the influence of depression and anxiety. Or, it may be that anxiety and depression are more predictive of self-damaging 
behaviors in clinical or college populations than they are in nonclinical, community populations.

Second, correlations were added among the error terms of the subscales of DTS, items of the ERS, and items on the Self-Damaging Behavior Questionnaire. The positive correlation between ERS items 14 and 15 may be due to a common theme of speed of experiencing emotions. The positive correlation between ERS items 15 and 19 may be due to a common theme of strength of emotional experiences. The negative correlation between DTS absorption and DTS Regulation differs from the direction of the correlation between them in the initial validation study by Simons and Gaher (2005) and thus requires further study. The positive correlation between two-week fasting and two-week limiting makes sense as both reflect an attempt to limit food intake. The positive correlation between two-week NSSI and two-week suicide attempts makes sense as both reflect actions which cause immediate harm to the body. In hindsight, some of these relationships may have been predictable based on existing literature and common content themes. Future researchers would do well to consider potential correlations among not only the latent variables in the model, but also the observed variables.

Third, a correlation was added between emotional reactivity and learned helplessness $(r=.53)$. Review of the extant literature failed to turn up a study of the relationship between general emotional reactivity and learned helplessness, so this is an important contribution. Though, subjects with MDD were found to demonstrate both heightened emotional reactivity to anticipated physical pain and heightened helplessness toward pain as compared to healthy controls (Strigo, Simmons, Matthews, Craig, \& Paulus, 2008). Additionally, Gignac, Cott, and Badley (2000) found perceived 
helplessness in managing arthritis and emotional reactivity to disabling or chronic health conditions were correlated $(r=.70)$. This current study revealed that emotional reactivity and learned helplessness are correlated in a sample of individuals with a history of selfdamaging behaviors. Further research is needed to learn if this relationship extends to the general population, yet the finding here extends it beyond those with MDD or a chronic health condition. While this relationship was not anticipated, it is not contrary to theory and, as such, was added to the model.

\section{Fitted Two-Week Model}

After the modifications discussed above, the resulting two-week model was well fit to the sample. Most dramatically, the hypothesized relationship between emotional reactivity, learned helplessness, and distress tolerance was strongly supported. Together, emotional reactivity and learned helplessness accounted for $70 \%$ of the variance in DT. Thus, as hypothesized, high emotional reactivity and high learned helplessness meaningfully explain low distress tolerance. This finding suggests that individuals who experience emotions intensely and for long durations and who also believe that they cannot influence change in their situations are likely to display poorer ability to tolerate distressing emotions.

Both emotional reactivity and learned helplessness each significantly explained variance in DT, confirming their roles as important individual difference factors in DT. While each contributed significantly, emotional reactivity explained approximately 6 times more variance in DT than learned helplessness explained. Therefore, emotional reactivity plays a critical role in explaining variance in DT. This is consistent with my hypotheses and the work of both Lynch and Mizon (2011) and Linehan (2015). 
Distress tolerance had a direct negative effect on two-week frequency of selfdamaging behaviors, explaining $7 \%$ of the variance. Given the daily variations in a multitude of variables that can occur over a two-week period, prediction of $7 \%$ of the variance in two-week frequency of self-damaging behaviors by stable factors which do not change from week to week is meaningful. However, as will be discussed below, less variance was explained in two-week frequency of self-damaging behaviors than in lifetime frequency of self-damaging behaviors. Further research is needed to explore the unexplained variance.

One possible statistical explanation for this finding is that the skewness and kurtosis of the two-week items remained even after log transformation of the values and this may have resulted in a latent variable which poorly represented the core concept of recent self-damaging behaviors. While the fit of the overall model indicated adequate fit for the Self-damaging Behavior Questionnaire measurement model, some of the individual items had small loadings onto the latent variable. Future researchers might seek a way to use frequency as an indicator of severity, while limiting the skewness and kurtosis of the resulting responses (e.g., through transformation to a categorical or Likert scale response).

Another possibility is that since DT is a relatively stable construct, it may not correspond with the two-week time frame of the behaviors. For example, individuals who have engaged in self-damaging behaviors in the past two-weeks may be doing so in response to a time-limited distressing event, but not have prolonged patterns of low DT over the course of their lifetimes. Future researchers might investigate the relationship between DT, as experienced in the prior two weeks, and two-week frequency of self- 
damaging behaviors. Another possibility is that the ceiling effect of the two-week time frame on the frequency of the behaviors may have muted the relationship with DT. Another possibility is that DT may be related to other variance in severity of recent selfdamaging behaviors (e.g., amount of weight lost in restricting, depth of cuts in NSSI, or lethality of method in suicide attempts), but not to variance in frequency.

\section{Lifetime Model Fit}

Structural equation modeling was used to evaluate whether the hypothesized relationships among variables in the lifetime frequency model were supported in this sample. Structural equation modeling indicated that the original model was a poor fit for the data, so revisions were made on the basis of the modification indices and theory.

Age did not remain a significant control in explaining variance in lifetime selfdamaging behaviors. This was surprising given that older individuals have had more years to accumulate frequency of self-damaging behaviors. Further research is needed to investigate the possible presence of a cohort effect or increasing trend in the frequency of self-damaging behaviors.

Anxiety also did not remain significant as a control and was removed. Thus, anxiety level did not explain variance in frequency of recent or lifetime self-damaging behaviors. So, further examination of the value of anxiety-reduction techniques in reducing self-damaging behaviors may be needed. However, other possibilities should also be considered and evaluated. For instance, my study assessed symptoms of anxiety over the past week. It may be that long-term experiences of anxiety may explain more variance in the self-damaging behaviors. Also, the measure of anxiety I used may have emphasized the physiological aspect of anxiety. So, another possibility is that the 
emotional or cognitive aspects of anxiety (e.g., AS) may show a stronger relationship with self-damaging behaviors than the physiological aspect did. Or, it may be that the relationship between AS and self-damaging behaviors reported in the extant literature is subsumed in the relationship between DT and self-damaging behaviors.

In contrast to the two-week model, depression did remain a significant control in the lifetime model. Thus, level of depression explained variance in lifetime frequency of self-damaging behaviors, but not in two-week frequency of self-damaging behaviors. With the retention of depression as a control, positive correlations were also added between emotional reactivity and depression and between learned helplessness and depression. While a review of these relationships is outside of the scope of this study, interested readers are encouraged to read Bylsma, Morris, and Rottenburg (2008) regarding the complex relationship between emotional reactivity and depression and Sweeney, Anderson, and Bailey (1986) regarding the nuanced relationship between learned helplessness and depression.

The correlations added between items and subscales in the two-week model, described above, were also added in the lifetime model. However, the correlation between lifetime NSSI and lifetime suicide attempts was not added as it was not recommended by IBM SPSS Amos 24 (Arbuckle, 2016) since including it would not improve the fit of the lifetime model. Thus, there is a stronger correlation between twoweek NSSI and two-week suicide attempts than between lifetime NSSI and lifetime suicide attempts. This suggests that clinicians should be particularly careful to assess for the co-occurrence of two-week NSSI and two-week suicide attempts. 


\section{Lifetime Fitted Model}

After the modifications discussed above, the resulting two-week model was well fit to the sample. As described above, emotional reactivity and learned helplessness together explained $70 \%$ of the variance in DT. In contrast to the two-week model, greater variance was explained in lifetime frequency of self-damaging behaviors. Distress tolerance had a direct negative effect on self-damaging behaviors and depression had a direct positive effect on self-damaging behaviors. Together, they explained $36 \%$ of the observed variance in lifetime frequency of self-damaging behaviors. This finding suggests that individuals with lowered mood and low ability to tolerate distressing emotions may engage in higher frequency of self-damaging behaviors over their lifetimes.

With a third of the variance in lifetime frequency of the self-damaging behaviors explained, this study significantly supports the important role of DT in self-damaging behaviors. Implications of this finding will be discussed below. Yet, there remains a significant portion of unexplained variance in the behaviors. Future researchers might use this model as a foundation and include additional variables which might contribute to explaining further variance in the self-damaging behaviors. Models which best represent human behavior are often complex and involve many variables, but this simpler model serves as an important foundation.

Some of the extant literature suggested a stronger link between DT and selfdamaging behaviors than was demonstrated in this study. It may be that DT explains greater variance in other aspects of distressing behaviors. For instance, DT may explain variance in severity in self-damaging behaviors beyond that represented by frequency 
(e.g. number of different methods of NSSI used). Another possibility is that difference in level of DT may explain variance between individuals who have a history of selfdamaging behaviors and individuals who do not (whereas everyone in my study had a history of self-damaging behaviors, which may have introduced a ceiling effect on DT). Or, the relationship between DT and self-damaging behaviors may be different in other populations studied in the extant literature such as clinical populations or adolescent populations. Additionally, the extant literature often studies DT in relation to diagnostic categories, rather than to only specific behaviors. So, it may be that DT explains even greater variance in diagnostic categories than it does in the self-damaging behaviors alone. For example, DT may explain more variance in the diagnostic category of AN than it does in restricting behaviors alone, since the diagnostic category also includes additional symptoms such as body dissatisfaction and fear of weight gain. This study thus demonstrated that DT and depression together explain an important portion of variance in the frequency of self-damaging behaviors.

Of note, lifetime restricting and lifetime binging did not load significantly onto the latent variable representing lifetime frequency of self-damaging behaviors and were thus removed from the model. This means that the results above speak only to the construct of self-damaging behaviors as represented by lifetime fasting, lifetime purging, lifetime NSSI, lifetime suicidal ideation, and lifetime suicide attempts. As with the twoweek model, some of the retained items had only small loadings on the latent variable. While the fit of the overall model confirms the fit of the measurement model for the Selfdamaging Behavior Questionnaire, it is unusual that the measurement model differs between two-week and lifetime frequency of the behaviors. This may be the result of low 
endorsement of some of the individual behaviors or the skewness and kurtosis of some of the two-week frequency items. Or, this may indicate that the individual behaviors studied do not represent a unified construct of self-damaging behaviors, so this construct will need to be examined in future research. In this study, items were drawn from two separate instruments to form the Self-damaging Behavior Questionnaire out of necessity as no unified scale existed to measure the frequency of a broad range of behaviors which are self-damaging. Future examination of the relationship between the behaviors in my study as well as other related behaviors which might cause long-term bodily harm (e.g., excessive exercise, misuse of illicit substances) might draw a clearer picture of the construct of self-damaging behaviors. This clearer picture would serve as a foundation for developing a perhaps more robust instrument.

Also, the relationship between DT and disordered eating behaviors was smaller in this study than reported in the extant literature. This may be due to the difference in sample, difference in wording of the behaviors assessed, difference in the statistical method, or difference in indicator of severity (frequency). Another option is that the cDTS developed by Corstorphine et al. (2007)which is used most commonly in the extant literature to study DT in relation to eating behaviors, might capture a slightly different construct than is captured by the sDTS which was used in this study. To see if this is true, future research could investigate the relationship between the cDTS and self-damaging behaviors using SEM.

\section{Limitations}

Several limitations should be considered with this study. Most notably, my study utilized a nonexperimental research design. As such, my results speak to correlation and 
prediction but not causation. However, a nonexperimental design was appropriate for my study since I desired to measure the variables as they are experienced in the respondents' daily contexts rather than in an experimental environment. Also, experimental manipulation of the variables of interest would have been unethical and dangerous given the nature of self-damaging behaviors.

The models tested were based on the literature review outlined in Chapter 2. The studies reviewed reflected greater representation of females than males and greater representation of Caucasians than other races. This may have been due to overrepresentation of females and Caucasians in the diagnoses (e.g., BPD), behaviors (e.g., disordered eating behaviors), or samples (e.g., clinical populations) of interest. While my survey was distributed to a representative distribution on the basis of sex and race, females and Caucasians were also overrepresented in my respondents. This was either due to more females and Caucasians clicking on the link to my survey or due to more females and Caucasians endorsing a lifetime history of the self-damaging behaviors on the screener question. Therefore, it is understood that my hypothesized models were drawn from literature which was not equally representative and my final models were revised based on respondents who were not equally representative. So, my results most fully describe females and Caucasians and will need to be replicated in different populations before results are generalized to these populations.

Also, all variables were assessed using self-report measures. This may have resulted in participants answering surveys in ways which were socially desirable, rather than in ways which accurately reflect their true behavior. However, the subjective nature of my variables required self-report. Given the sensitive nature of the variables of interest 
in this study, anonymous surveys may have allowed for more open sharing of risky behaviors than other data collection methods would have facilitated. Additionally, my research was intended to be applicable in clinical work in which a clinician's data is primarily client self-report through narrative or brief assessments.

Further, since my survey was hosted online, it limited my sample to individuals who had internet access, were comfortable with the use of the internet, and who had signed up specifically for completing online surveys through QuestionPro. It may be that individuals who voluntarily sign up to complete online surveys differ from the general population in their levels of the variables of interest. However, the online platform allowed for data collection from respondents who are heterogeneous in age, ethnicity, geographic location, SES, and sex. Additionally, the vast majority of research on DT has been conducted with undergraduate or clinical populations, so it was important to survey a nonclinical population outside a college setting in order to broaden the literature.

Additionally, I analyzed my data using SEM. So, my results speak to the degree of fit observed between my resulting SEM models and the data from the current sample. It will always be possible that different models (including different variables or the same variables arranged in a different configuration) would be an even better fit for the data. Structural equation modeling was a good analysis approach for my research questions since it allowed for the assessment of multiple latent variables simultaneously.

Another limitation of my study was the skewness and kurtosis of the items on the Self-Damaging Behavior Questionnaire. While this is reflective of lived experience, it resulted in the need to log transform the responses before SEM was conducted. Some of the two-week frequency items demonstrated continued skewness or kurtosis even after 
$\log$ transformation. The impact of this on the results of the two-week model is unknown and requires further evaluation.

Further, while the measurement model for the Self-damaging Behavior Questionnaire showed adequate fit in the individual models, it differed between the twoweek and the lifetime models. Combined with low loadings of some of the items onto the latent variables, this raises the question of whether or not self-damaging behaviors represent a unified construct. Further study of the construct is needed. However, the questionnaire was developed out of necessity as a unified measure of frequency of a variety of self-damaging behaviors did not exist.

\section{Recommendations}

Until this point, research and practice has been limited by lack of knowledge of individual difference factors in DT. Therefore, my models of the relationship between emotional reactivity, learned helplessness, DT, depression, and self-damaging behaviors makes an important contribution to advancing understanding in the field. Implications of my findings for both practice and further research are listed below.

\section{Recommendations for Practice}

1. Given the high overall endorsement of self-damaging behaviors (as indicated by $61 \%$ of potential participants endorsing history of at least one self-damaging behavior), all clinicians should screen for current self-damaging behaviors in their clients. If not already included in the intake paperwork, formal assessment through the EDE-Q (Fairburn \& Beglin, 2008) or SITBI (Nock et al., 2007) might be beneficial. Clinicians should be particularly careful to assess for the potential 
co-occurrence of NSSI and suicide attempts in the past two weeks (e.g., if a client endorses NSSI within the past two weeks, the clinician should also explicitly assess for any suicide attempts in that time period).

2. In recognition of the strong role of emotional reactivity in predicting low DT, clinicians should consider targeting level of emotional reactivity through clinical interventions. For instance, biofeedback (Allen, Harmon-Jones, \& Cavender, 2001), DBT mindfulness practice (Feliu-Soler et al., 2014), mindfulness meditation (Creswell, Way, Eisenberger, \& Lieberman, 2007), MindfulnessBased Cognitive Therapy (Britton, Shahar, Szepsenwol, \& Jacobs, 2012), Mindfulness-Based Stress Reduction (Goldin \& Gross, 2010), and selfcompassion (Leary, Tate, Adams, Allen, \& Hancock, 2007) have all demonstrated promise in reducing emotional reactivity which in turn should improve DT according to the results of this study.

3. In recognition of the strong role of learned helplessness in predicting low DT, clinicians should consider targeting learned helplessness through clinical interventions. This may include utilizing a scaffolding approach to tasks which provide optimal opportunity to learn agency and see the successful impact of one's own actions on the situation (i.e., set up small tasks for which the client is very likely to experience success). Therapists might also encourage active problem solving within session to counter beliefs about nonagency. This may include targeting cognitive attributions which perpetuate and generalize learned helplessness. For information about interventions which target change in attributional style, see Peterson et al. (1993), Proudfoot, Corr, Guest, and Dunn 
(2009), and Rubenstein, Freed, Shapero, Fauber, and Alloy (2016). For information about interventions which build hope and persistence to counter learned helplessness, see Nation and Massad (1978), Levine, Irving, Brooks, and Fishman (1993), Peterson and Seligman (2004), and Maier and Seligman (2016). For information about interventions which build perceived control and mastery to counter learned helplessness, see Skinner (1995), Zautra et al. (2012), and Nguyen-Feng et al. (2015).

4. Given the strong relationship between depression and lifetime frequency of selfdamaging behaviors, clinicians should consider assessing and targeting depression when treating clients with self-damaging behaviors. Since a pessimistic attribution style (attributing negative events to internal, stable, and global factors) is known to be associated with depression (Abramson, Seligman, \& Teasdale, 1978; Sweeney et al., 1986), the interventions noted above which target change in attribution style may simultaneously impact both learned helplessness and depression (see Rubenstein et al., 2016). For current best practice guidelines for the treatment of depression, see American Psychiatric Association (2010). For a network meta-analysis of the efficacy of different psychotherapy approaches in the treatment of depression, see Barth et al. (2013). For an overview of relapse prevention in depression, see Richards and Perri (2010). For guidelines in selecting an evidence-based practice in the treatment of older adults, see Substance Abuse and Mental Health Services Administration (2011).

5. In light of the strong role of DT in lifetime frequency of self-damaging behaviors and moderate role of DT in two-week frequency of self-damaging behaviors, 
clinicians should consider incorporating interventions which target increasing DT as a way to decrease the factors related to self-damaging behaviors. For information about an intensive, short term, CBT-based intervention which targets DT, see Williams et al. (2013), and for an online, short term, CBT-based intervention which targets DT, see McHugh et al. (2014). For information about a group intervention based on DBT which targets DT, see Booth et al. (2014). For information about an intervention developed specifically to target DT through emotional exposure, see Bornovalova et al. (2012).

6. The self-damaging behaviors studied represented a broad range of behaviors which may or may not fit neatly into existing mental health diagnoses. Thus, the clinical recommendations noted above are transdiagnostic. That is, the interventions are relevant to reducing self-damaging behaviors regardless of what specific diagnosis an individual has or does not have. This study suggests that it may be more relevant to the treatment of self-damaging behaviors to identify a client's level of emotional reactivity, learned helplessness, and DT than to identify a specific diagnosis.

\section{Recommendations for Future Research}

1. Future research is needed to determine whether the incidence rate of selfdamaging behaviors in this survey is true of the general population, or is unique to an online population.

2. This study contributed to the literature the overall incidence rate of self-damaging behaviors in a nonclinical sample. While more research has examined individual self-damaging behaviors in clinical samples than in nonclinical samples, less is 
known about the overall incidence rate of these behaviors. So, future research could investigate the overall incidence rate of self-damaging behaviors in clinical populations.

3. This study assumed a continuum between learned industriousness and learned helplessness. Future research could more thoroughly investigate for the presence of this continuum, while avoiding competing ceiling and floor effects. Or, future research could investigate whether learned industriousness (as in the original theory of Lynch \& Mizon, 2011) might explain even more variance in DT than learned helplessness.

4. This research was limited by the skewness and kurtosis of the reported frequency of self-damaging behavior data. While representative of the natural occurrence of these behaviors, the skewness and kurtosis exceeded that which can be managed by SEM in IBM SPSS Amos 24 (Arbuckle, 2016). Future researchers might consider (a) using a statistical method more robust to skewness and kurtosis, (b) reducing frequency responses to categorical options or a Likert scale, or (c) using a different indicator of severity.

5. While emotional reactivity and learned helplessness accounted for $70 \%$ of the variance in DT, additional variance remains unexplained. In the interest of simplicity and developing a foundational model, and because no other variables were clearly recommended by the current literature, only two exogenous variables were included in the hypothesized models in this study. Future researchers could test a more expansive and explanatory model that includes more exogenous variables that the literature has not yet identified but which may be relevant, such 
as certain demographic variables, personality traits, or adverse childhood experiences.

6. This study examined the relationship between emotional reactivity, learned helplessness, and self-damaging behaviors and a latent variable representing overall DT. It may be that different relationships exist between the variables and the different subscales of DT (absorption, appraisal, regulation, and tolerance). Future researchers could investigate the pattern of relationships among the variables and these sub-constructs.

7. The Self-damaging Behavior Questionnaire was compiled for this study because a single instrument measuring the frequency of the self-damaging behaviors of interest did not exist. While overall reliability of the Self-damaging Behavior Questionnaire was acceptable, the reliability of some of the individual items was low. Future research could refine this instrument or develop a different instrument to assess the frequency of a broad range of self-damaging behaviors.

8. While initially distributed to a representative population, the sample in this study largely represented Caucasian females. This likely speaks to lifetime incidence of self-damaging behaviors being higher in this demographic group. However, future research is needed to test how this model applies to broader demographics.

9. This study utilized an adult sample. However, self-damaging behaviors also impact teenage populations, possibly at higher frequencies. Further research is needed to test whether this model applies to younger individuals. This may be particularly important as the incidence of self-damaging behaviors is thought to be increasing among teenagers. 
10. Having highlighted both emotional reactivity and learned helplessness as individual difference factors in DT, future research could test the impact of individual or group interventions targeted at reducing emotional reactivity and learned helplessness on DT.

\section{Summary}

In summary, this research showed that emotional reactivity and learned helplessness significantly predicted $70 \%$ of the variance in individuals' levels of DT. The results also showed that DT significantly predicted $7 \%$ of the variance in two-week frequency of self-damaging behaviors. Distress tolerance, together with depression, also predicted $36 \%$ of the variance in lifetime frequency of self-damaging behaviors. A number of clinically relevant interventions were suggested that could reduce selfdamaging behaviors regardless of what diagnoses clients may exhibit. Finally, recommendations for additional research outlined a research agenda for the future that is likely to make additional contributions to the field for years to come. 
APPENDIX A

STUDIES OF THE RELATIONSHIP BETWEEN DT AND SELF-DAMAGING BEHAVIORS 


\begin{tabular}{|c|c|c|c|}
\hline $\begin{array}{c}\text { AUTHOR(S) and } \\
\text { TITLE }\end{array}$ & $\begin{array}{c}\text { DESIGN AND } \\
\text { ANALYSIS }\end{array}$ & $\begin{array}{c}\text { MEASURE OF DT } \\
\text { and SAMPLE }\end{array}$ & RESULTS \\
\hline \multicolumn{4}{|c|}{ ANOREXIA NERVOSA } \\
\hline $\begin{array}{l}\text { (Hambrook et al., } \\
\text { 2011) } \\
\text { Emotional } \\
\text { expression, self- } \\
\text { silencing, and distress } \\
\text { tolerance in anorexia } \\
\text { nervosa and chronic } \\
\text { fatigue syndrome }\end{array}$ & $\begin{array}{l}\text { Cross-sectional: One- } \\
\text { time survey collection. } \\
\text { Analysis by correlation, } \\
\text { ANOVAs, and t-tests. }\end{array}$ & $\begin{array}{l}\text { Emotional self-report: } \\
\text { cDTS } \\
+\mathrm{N}=40 ; \text { Outpatients } \\
\text { with AN. } \\
\mathrm{N}=45 \text {; Outpatients } \\
\text { with chronic fatigue } \\
\text { syndrome. } \\
\mathrm{N}=47 \text {; Healthy } \\
\text { controls. }\end{array}$ & $\begin{array}{l}\text { Both outpatient groups scored higher than healthy } \\
\text { controls on the DT Avoidance of Affect subscale, } \\
\text { but the difference was insignificant after controlling } \\
\text { for age, anxiety, and depression. }\end{array}$ \\
\hline $\begin{array}{l}\text { (Oldershaw et al., } \\
\text { 2012) } \\
\text { Emotional processing } \\
\text { following recovery } \\
\text { from anorexia } \\
\text { nervosa }\end{array}$ & $\begin{array}{l}\text { Cross-sectional: One- } \\
\text { time survey collection. } \\
\text { Analysis by correlation, } \\
\text { ANOVAs, and t-tests. }\end{array}$ & $\begin{array}{l}\text { Emotional self-report: } \\
\text { cDTS } \\
+\mathrm{N}=40 \text {; Outpatients } \\
\text { with current AN. } \\
\mathrm{N}=24 \text {; Community } \\
\text { with prior AN. } \\
\mathrm{N}=48 \text {; Matched } \\
\text { healthy controls. }\end{array}$ & $\begin{array}{l}\text { Current AN group reported higher on cDTS: } \\
\text { Avoidance of Affect subscale than both prior AN } \\
\text { and control groups. Prior AN group and control } \\
\text { group did not differ on cDTS scores. }\end{array}$ \\
\hline \multicolumn{4}{|l|}{ ANXIETY } \\
\hline $\begin{array}{l}\text { (Fergus et al., 2015) } \\
\text { Examining the } \\
\text { specific facets of } \\
\text { distress tolerance that } \\
\text { are relevant to health } \\
\text { anxiety }\end{array}$ & $\begin{array}{l}\text { Cross-sectional: One- } \\
\text { time survey collection. } \\
\text { Analysis by structural } \\
\text { equation model and } \\
\text { structural regression } \\
\text { model. }\end{array}$ & $\begin{array}{l}\text { Emotional self-report: } \\
\text { sDTS } \\
\text { Physical self-report, } \\
\text { DIS } \\
\text { N }=830 \text {; Nonclinical. }\end{array}$ & $\begin{array}{l}\text { DT was negatively correlated with health anxiety. } \\
\text { DT was uniquely predictive of health anxiety while } \\
\text { also considering IU, TA, FT, and DI. However, IU } \\
\text { and DI accounted for greater variance in health } \\
\text { anxiety than DT. }\end{array}$ \\
\hline
\end{tabular}




\begin{tabular}{|c|c|c|c|}
\hline $\begin{array}{l}\text { (Huang et al., 2009) } \\
\text { The relationship of } \\
\text { low distress tolerance } \\
\text { to excessive worrying } \\
\text { and cognitive } \\
\text { avoidance }\end{array}$ & $\begin{array}{l}\text { Cross-sectional: } \\
\text { Completion of a } \\
\text { Thought-listing task, } \\
\text { then one-time survey } \\
\text { collection. Analysis by } \\
\text { correlation and } \\
\text { hierarchical multiple } \\
\text { linear regression. }\end{array}$ & $\begin{array}{l}\text { Emotional self-report: } \\
\text { sDTS } \\
\mathrm{N}=119 \\
\text { Undergraduates. }\end{array}$ & $\begin{array}{l}\text { DT was negatively correlated with worry, } \\
\text { depression, anxiety, and stress. DT was associated } \\
\text { with worry, after controlling for depression, anxiety, } \\
\text { and stress. DT was negatively correlated with } \\
\text { concreteness during worrying. DT was not } \\
\text { correlated with imagery. }\end{array}$ \\
\hline $\begin{array}{l}\text { (Johnson et al., 2012) } \\
\text { Nonclinical panic } \\
\text { attack history and } \\
\text { anxiety sensitivity: } \\
\text { Testing the } \\
\text { differential } \\
\text { moderating role of } \\
\text { self-report and } \\
\text { behavioral indices of } \\
\text { distress tolerance }\end{array}$ & $\begin{array}{l}\text { Cross-sectional: One- } \\
\text { time survey collection } \\
\text { followed by behavioral } \\
\text { task. Analysis by } \\
\text { correlation and } \\
\text { hierarchical linear } \\
\text { regression. }\end{array}$ & $\begin{array}{l}\text { Emotional self-report: } \\
\text { sDTS } \\
\text { Physical behavioral: } \\
\text { BHD } \\
\mathrm{N}=145 \text {; Nonclinical } \\
\text { with half of the sample } \\
\text { meeting criteria for } \\
\text { nonclinical panic } \\
\text { attacks within the past } \\
2 \text { years and half of the } \\
\text { sample having no } \\
\text { lifetime history of } \\
\text { panic attacks. }\end{array}$ & $\begin{array}{l}\text { Self-reported DT, but not behavioral DT, predicted } \\
\text { variance in total AS, physical AS, cognitive AS, and } \\
\text { social AS after controlling for neuroticism, sex, and } \\
\text { panic attack history. Nonclinical panic attack history } \\
\text { and breath holding duration interacted in the } \\
\text { prediction of physical AS, such that the panic attack } \\
\text { history and physical AS relationship was stronger } \\
\text { for individuals with low levels of DT. Nonclinical } \\
\text { panic attack history and self-reported DT interacted } \\
\text { in the prediction of cognitive AS, such that the panic } \\
\text { attack history and cognitive AS relationship was } \\
\text { stronger for individuals with low levels of DT. }\end{array}$ \\
\hline $\begin{array}{l}\text { (Keough et al., 2010) } \\
\text { Anxiety } \\
\text { symptomatology: The } \\
\text { association with } \\
\text { distress tolerance and } \\
\text { anxiety sensitivity }\end{array}$ & $\begin{array}{l}\text { Cross-sectional: One- } \\
\text { time survey collection. } \\
\text { Analysis by } \\
\text { hierarchical linear } \\
\text { regression and } \\
\text { moderational analyses. }\end{array}$ & $\begin{array}{l}\text { Emotional self-report: } \\
\text { sDTS } \\
\mathrm{N}=418 \\
\text { Undergraduates. }\end{array}$ & $\begin{array}{l}\text { DT was associated with OCD, panic, anxious worry, } \\
\text { and social anxiety symptoms, after controlling for } \\
\text { AS, anxiety, and depression. DT was negatively } \\
\text { correlated with AS. The interaction between DT and } \\
\text { AS was not associated with the outcome measures. }\end{array}$ \\
\hline
\end{tabular}




\begin{tabular}{|c|c|c|c|}
\hline \multirow{2}{*}{$\begin{array}{l}\text { (Kertz et al., 2015) } \\
\text { Distress intolerance } \\
\text { and worry: The } \\
\text { mediating role of } \\
\text { cognitive variables }\end{array}$} & $\begin{array}{l}\text { Study 1: } \\
\text { Cross-sectional; One- } \\
\text { time survey collection. } \\
\text { Analysis by multiple } \\
\text { mediator models. }\end{array}$ & $\begin{array}{l}\text { Emotional self-report: } \\
\text { DII } \\
\mathrm{N}=281 \\
\text { Undergraduates. }\end{array}$ & $\begin{array}{l}\text { Lower levels of DT were associated with higher } \\
\text { levels of worry. This relationship was partially } \\
\text { mediated by both positive and negative beliefs about } \\
\text { worry. }\end{array}$ \\
\hline & $\begin{array}{l}\text { Study 2: } \\
\text { Cross-sectional; One- } \\
\text { time survey collection. } \\
\text { Analysis by multiple } \\
\text { mediator models. }\end{array}$ & $\begin{array}{l}\text { Emotional self-report: } \\
\text { DII } \\
\text { N = 123; Inpatients. }\end{array}$ & $\begin{array}{l}\text { Lower levels of DT were associated with higher } \\
\text { levels of worry. The relationship between worry and } \\
\text { DT was partially mediated by negative problem } \\
\text { orientation and negative beliefs about worry. }\end{array}$ \\
\hline $\begin{array}{l}\text { (Kutz et al., 2010) } \\
\text { Evaluating emotional } \\
\text { sensitivity and } \\
\text { tolerance factors in } \\
\text { the prediction of } \\
\text { panic-relevant } \\
\text { responding to a } \\
\text { biological challenge }\end{array}$ & $\begin{array}{l}\text { Cross-sectional: } \\
\text { Baseline survey } \\
\text { collection, then Carbon } \\
\text { dioxide-enriched air } \\
\text { challenge, then } \\
\text { assessment of panic } \\
\text { symptoms. Analysis by } \\
\text { correlation and } \\
\text { hierarchical linear } \\
\text { regression. }\end{array}$ & $\begin{array}{l}\text { Emotional self-report: } \\
\text { sDTS } \\
\text { Physical self-report: } \\
\text { DIS } \\
\mathrm{N}=219 \text {; Nonclinical. }\end{array}$ & $\begin{array}{l}\text { Neither DT nor DI were associated with post- } \\
\text { challenge panic attack status, cognitive panic } \\
\text { symptoms, or physical panic symptoms after } \\
\text { controlling for recent panic attack history, negative } \\
\text { affect, and AS. }\end{array}$ \\
\hline
\end{tabular}




\begin{tabular}{|c|c|c|c|}
\hline $\begin{array}{l}\text { (Leyro et al., 2012) } \\
\text { Evaluation of } \\
\text { perseveration in } \\
\text { relation to panic- } \\
\text { relevant responding: } \\
\text { An initial test }\end{array}$ & $\begin{array}{l}\text { Cross-sectional: } \\
\text { Baseline surveys and } \\
\text { emotional challenge } \\
\text { administered, then } \\
\text { Carbon dioxide- } \\
\text { enriched air challenge } \\
\text { (within } 2 \text { weeks), then } \\
\text { assessment of panic } \\
\text { symptoms. Analysis by } \\
\text { correlation, ANOVA, } \\
\text { and hierarchical linear } \\
\text { regression. }\end{array}$ & $\begin{array}{l}\text { Emotional self-report: } \\
\text { sDTS } \\
\text { Emotional behavioral: } \\
\text { MTPT } \\
\mathrm{N}=88 \text {; Nonclinical } \\
\text { with half of the sample } \\
\text { meeting criteria for } \\
\text { nonclinical panic } \\
\text { attacks within the past } \\
2 \text { years and half of the } \\
\text { sample having no } \\
\text { lifetime history of } \\
\text { panic attacks. }\end{array}$ & $\begin{array}{l}\text { Neither self-report nor discontinue time on the } \\
\text { MTPT were predictive of post challenge panic } \\
\text { symptoms, after controlling for negative affect, sex, } \\
\text { panic attack history, number of axis } 1 \text { diagnoses, } \\
\text { perseveration, perfectionism, and persistence. }\end{array}$ \\
\hline \multirow{2}{*}{$\begin{array}{l}\text { (Norr et al., 2013) } \\
\text { Evaluating the unique } \\
\text { contribution of } \\
\text { intolerance of } \\
\text { uncertainty relative to } \\
\text { other cognitive } \\
\text { vulnerability factors } \\
\text { in anxiety } \\
\text { psychopathology }\end{array}$} & $\begin{array}{l}\text { Study 1: } \\
\text { Cross-sectional: One- } \\
\text { time survey collection. } \\
\text { Analysis by correlation } \\
\text { and hierarchical linear } \\
\text { regression. }\end{array}$ & $\begin{array}{l}\text { Emotional self-report: } \\
\text { sDTS } \\
\text { Physical self-report: } \\
\text { DIS } \\
\mathrm{N}=217 \text {; } \\
\text { Undergraduates. }\end{array}$ & $\begin{array}{l}\text { DT and DI were not predictive of social anxiety or } \\
\text { non-hoarding OCD symptoms, after controlling for } \\
\text { negative affect, sex, AS, and IU. DT was predictive } \\
\text { of general worry with the same controls, but DI was } \\
\text { not. }\end{array}$ \\
\hline & $\begin{array}{l}\text { Study 2: } \\
\text { Cross-sectional: One- } \\
\text { time survey collection. } \\
\text { Analysis by correlation } \\
\text { and hierarchical linear } \\
\text { regression. }\end{array}$ & $\begin{array}{l}\text { Emotional self-report: } \\
\text { sDTS } \\
\text { Physical self-report: } \\
\text { DIS } \\
\mathrm{N}=241 \text {; } \\
\text { Undergraduates. }\end{array}$ & $\begin{array}{l}\text { DT and DI were not predictive of social anxiety, } \\
\text { non-hoarding OCD symptoms, or worry after } \\
\text { controlling for trait anxiety, sex, AS, and IU. }\end{array}$ \\
\hline
\end{tabular}




\begin{tabular}{|c|c|c|c|}
\hline \multicolumn{4}{|l|}{ BULIMIA NERVOSA } \\
\hline $\begin{array}{l}\text { (Anestis et al., 2007) } \\
\text { The multifaceted role } \\
\text { of distress tolerance } \\
\text { in dysregulated } \\
\text { eating behaviors }\end{array}$ & $\begin{array}{l}\text { Cross-sectional: One } \\
\text { time survey collection. } \\
\text { Analysis by correlation, } \\
\text { linear regression, and } \\
\text { mediation analysis. }\end{array}$ & $\begin{array}{l}\text { Emotional self-report: } \\
\text { sDTS } \\
\mathrm{N}=200 \\
\text { Undergraduates. }\end{array}$ & $\begin{array}{l}\text { DT predicted Bulimic symptoms when controlling } \\
\text { for sex, depression, anxiety, AS, negative affect, } \\
\text { urgency, sensation seeking, lack of premediation, } \\
\text { lack of perseverance, interoceptive awareness, } \\
\text { perfectionism, drive for thinness, and body } \\
\text { dissatisfaction. Additionally, DT and negative } \\
\text { urgency interacted in the prediction of bulimic } \\
\text { symptoms such that individuals with low DT and } \\
\text { high negative urgency were most likely to report } \\
\text { high levels of bulimic symptoms. DT also mediated } \\
\text { the relationship between AS and bulimic symptoms. }\end{array}$ \\
\hline $\begin{array}{l}\text { (Lavender et al., } \\
\text { 2015) } \\
\text { The interactive role } \\
\text { of distress tolerance } \\
\text { and eating } \\
\text { expectancies in } \\
\text { bulimic symptoms } \\
\text { among substance } \\
\text { abusers }\end{array}$ & $\begin{array}{l}\text { Cross-sectional: One- } \\
\text { time survey collection. } \\
\text { Analysis by correlation } \\
\text { and hierarchical } \\
\text { multiple regression } \\
\text { analysis. }\end{array}$ & $\begin{array}{l}\text { Emotional self-report: } \\
\text { sDTS } \\
\mathrm{N}=93 \text {; Inpatients } \\
\text { with SUD. }\end{array}$ & $\begin{array}{l}\text { DT, Negative Affect Eating Expectancy, and the } \\
\text { interaction between DT and Negative Affect Eating } \\
\text { Expectancy were predictive of bulimic symptoms, } \\
\text { after controlling for sex and overall negative affect. } \\
\text { The interaction was such that the magnitude of the } \\
\text { association between DT and bulimic symptoms was } \\
\text { greater at moderate levels of Negative Affect Eating } \\
\text { Expectancy than at low levels. }\end{array}$ \\
\hline
\end{tabular}




\begin{tabular}{|c|c|c|c|}
\hline \multicolumn{4}{|l|}{ DEPRESSION } \\
\hline \multirow{2}{*}{$\begin{array}{l}\text { (Williams et al., } \\
\text { 2013) } \\
\text { The impact of } \\
\text { psychological distress } \\
\text { tolerance in the } \\
\text { treatment of } \\
\text { depression }\end{array}$} & $\begin{array}{l}\text { Study } 1: \text { Pre- and post- } \\
\text { treatment survey } \\
\text { collection. Treatment } \\
\text { was } 6 \text { online CBT } \\
\text { sessions over a 10- } \\
\text { week time period. } \\
\text { Analysis by marginal } \\
\text { model analysis and } \\
\text { correlations. }\end{array}$ & $\begin{array}{l}\text { Emotional self-report: } \\
\text { sDTS } \\
\mathrm{N}=75 \text {; Outpatients in } \\
\text { Australia who met } \\
\text { probable diagnosis of } \\
\text { depression. }\end{array}$ & $\begin{array}{l}\text { sDTS: Absorption, sDTS: Appraisal, and sDTS: } \\
\text { Tolerance subscales all decreased between pre- and } \\
\text { post-treatment assessment. Time and total DT (but } \\
\text { not the interaction between them) were significant } \\
\text { predictors of post-treatment depression and } \\
\text { psychological distress. Individuals with low DT at } \\
\text { baseline had higher baseline and post-treatment } \\
\text { depression and psychological distress compared to } \\
\text { individuals with high DT at baseline. }\end{array}$ \\
\hline & $\begin{array}{l}\text { Study 2: Pre- and post- } \\
\text { treatment sDTS } \\
\text { collection. Same } \\
\text { treatment as Study 1, } \\
\text { except half received via } \\
\text { smart phone. Analysis } \\
\text { by marginal model } \\
\text { analysis and } \\
\text { correlations. }\end{array}$ & $\begin{array}{l}\text { Emotional self-report: } \\
\text { sDTS, only tolerance } \\
\text { and regulation } \\
\text { subscales } \\
\mathrm{N}=35 \text {; Nonclinical } \\
\text { individuals who met } \\
\text { criteria for current } \\
\text { MDD. }\end{array}$ & $\begin{array}{l}\text { Time, sDTS: Tolerance, and sDTS: Regulation were } \\
\text { significant predictors of post-treatment depression } \\
\text { and psychological distress. Both sDTS: Tolerance } \\
\text { and sDTS: Regulation increased from baseline to } \\
\text { post-treatment. sDTS: Tolerance and sDTS: } \\
\text { Regulation were negatively correlation with ratings } \\
\text { of homework effort (but not amount of homework } \\
\text { completed) across the program. }\end{array}$ \\
\hline \multicolumn{4}{|c|}{ DEPRESSION AND ANXIETY } \\
\hline $\begin{array}{l}\text { (Allan et al., 2014) } \\
\text { Direct and interactive } \\
\text { effects of distress } \\
\text { tolerance and anxiety } \\
\text { sensitivity on } \\
\text { generalized anxiety } \\
\text { and depression }\end{array}$ & $\begin{array}{l}\text { Cross-sectional: One- } \\
\text { time survey collection. } \\
\text { Analysis by CFA and } \\
\text { SEM. }\end{array}$ & $\begin{array}{l}\text { Emotional self-report: } \\
\text { sDTS N = 347; } \\
\text { Outpatients. }\end{array}$ & $\begin{array}{l}\text { DT accounted for variance in worry and GAD } \\
\text { diagnoses at low and average AS levels, but not at } \\
\text { high levels. AS accounted for the most variance in } \\
\text { worry, GAD diagnosis, and MDD diagnosis at high } \\
\text { levels of DT. DT accounted for variance in MDD } \\
\text { diagnosis only at low levels of AS. DT did not } \\
\text { account for variance in depression symptoms, after } \\
\text { controlling for worry, sex, and AS. }\end{array}$ \\
\hline
\end{tabular}




\begin{tabular}{|c|c|c|c|}
\hline $\begin{array}{l}\text { (Norr et al., 2014) } \\
\text { The effects of an } \\
\text { anxiety sensitivity } \\
\text { intervention on } \\
\text { anxiety, depression, } \\
\text { and worry: mediation } \\
\text { through affect } \\
\text { tolerances }\end{array}$ & $\begin{array}{l}\text { Experimental: Pre- } \\
\text { treatment, one week } \\
\text { post-treatment, and one } \\
\text { month post-treatment } \\
\text { survey collection. } \\
\text { Treatment was a single } \\
\text { psychoeducational } \\
\text { group focused on AS } \\
\text { reduction (experimental } \\
\text { group) or physical } \\
\text { health education } \\
\text { (control group). } \\
\text { Analysis through } \\
\text { correlation, regression } \\
\text { analysis, and mediation } \\
\text { models. }\end{array}$ & $\begin{array}{l}\text { Emotional self-report: } \\
\text { sDTS } \\
\text { Physical self-report: } \\
\text { DIS } \\
\mathrm{N}=104 \text {; } \\
\text { Undergraduates with } \\
\text { elevated AS. (52 in } \\
\text { control and } 52 \text { in } \\
\text { experimental group) }\end{array}$ & $\begin{array}{l}\text { Pre-intervention DT was negatively correlated with } \\
\text { pre-intervention AS, DI, worry, anxiety, and } \\
\text { depression symptoms. The intervention increased } \\
\text { week } 1 \text { DT, but not DI. Week } 1 \text { DT fully mediated } \\
\text { the relationship between the intervention and 1- } \\
\text { month worry and depression symptoms and partially } \\
\text { mediated the relationship with anxiety symptoms. } \\
\text { However, when AS was added as an additional } \\
\text { mediation pathway, the mediation effect of DT was } \\
\text { no longer significant. }\end{array}$ \\
\hline \multicolumn{4}{|c|}{ EATING DISORDER GENERAL } \\
\hline $\begin{array}{l}\text { (Corstorphine et al., } \\
\text { 2007) } \\
\text { Distress tolerance in } \\
\text { the eating disorders }\end{array}$ & $\begin{array}{l}\text { Cross-sectional: One- } \\
\text { time survey collection. } \\
\text { Analysis by EFA, t- } \\
\text { tests, and correlations. }\end{array}$ & $\begin{array}{l}\text { Emotional self-report: } \\
\text { cDTS } \\
++\mathrm{N}=62 ; \text { Matched } \\
\text { undergraduate and } \\
\text { graduate females in } \\
\text { Britain without history } \\
\text { of an ED. } \\
\mathrm{N}=72 ; \text { Female } \\
\text { inpatients with an ED }\end{array}$ & $\begin{array}{l}\text { The clinical group scored higher on cDTS: } \\
\text { Avoidance of Affect. The nonclinical group scored } \\
\text { higher on cDTS: Accept and Manage. The groups } \\
\text { did not differ on cDTS: Anticipate and Distract. In } \\
\text { the nonclinical group, Bulimia symptoms were } \\
\text { positively correlated with cDTS: Avoidance of } \\
\text { Affect. In the clinical group, body dissatisfaction } \\
\text { symptoms were positively correlated with cDTS: } \\
\text { Avoidance of Affect and negatively correlated with } \\
\text { cDTS: Accept and Manage. }\end{array}$ \\
\hline
\end{tabular}




\begin{tabular}{|c|c|c|c|}
\hline $\begin{array}{l}\text { (Lampard et al., } \\
\text { 2011) } \\
\text { Avoidance of affect } \\
\text { in the eating } \\
\text { disorders }\end{array}$ & $\begin{array}{l}\text { Cross-sectional: One- } \\
\text { time online survey } \\
\text { collection. Analysis by } \\
\text { EFA, CFA, } \\
\text { correlations, and t-tests. }\end{array}$ & $\begin{array}{l}\text { Emotional self-report: } \\
\text { Eight items from } \\
\text { cDTS } \\
\mathrm{N}=227 \\
\text { Undergraduate } \\
\text { females. } \\
\mathrm{N}=257 \text {; Outpatient } \\
\text { females in Australia. }\end{array}$ & $\begin{array}{l}\text { Overall ED symptoms were positively correlated } \\
\text { with cDTS: Behavioral Avoidance of Positive Affect } \\
\text { and cDTS: Behavioral Avoidance of Negative affect } \\
\text { in both samples. In the nonclinical sample, cDTS: } \\
\text { Cognitive avoidance of Negative Affect was also } \\
\text { positively correlated with overall ED symptoms. In } \\
\text { the same sample, cDTS: Behavioral Avoidance of } \\
\text { Positive Affect was positively correlated with } \\
\text { purging symptoms and cDTS: Behavioral } \\
\text { Avoidance of Negative Affect was positively } \\
\text { correlated with binge eating. The clinical sample } \\
\text { scored higher on cDTS: Behavioral Avoidance of } \\
\text { Positive Affect and lower on cDTS: Cognitive } \\
\text { Avoidance of Negative Affect. }\end{array}$ \\
\hline $\begin{array}{l}\text { (Lydecker et al., } \\
\text { 2014) } \\
\text { White public regard: } \\
\text { Associations among } \\
\text { eating disorder } \\
\text { symptomatology, } \\
\text { guilt, and White guilt } \\
\text { in young adult } \\
\text { women }\end{array}$ & $\begin{array}{l}\text { Cross-sectional: One- } \\
\text { time survey collection. } \\
\text { Analysis by correlation } \\
\text { and multiple linear } \\
\text { regression. }\end{array}$ & $\begin{array}{l}\text { Emotional self-report: } \\
\text { sDTS } \\
\mathrm{N}=347 \\
\text { Undergraduate } \\
\text { females. }\end{array}$ & $\begin{array}{l}\text { DT was negatively associated with disordered } \\
\text { eating. DT moderated the relationship between white } \\
\text { guilt and disinhibited eating symptoms as well as } \\
\text { desire to be thinner. DT did not moderate } \\
\text { relationship between white guilt and restraint, } \\
\text { hunger, body dissatisfaction, or bulimic symptoms. } \\
\text { The association between white guilt at eating } \\
\text { symptoms was greater at low levels of DT. }\end{array}$ \\
\hline
\end{tabular}




\begin{tabular}{|c|c|c|c|}
\hline $\begin{array}{l}\text { (Mountford, } \\
\text { Corstorphine, } \\
\text { Tomlinson, \& } \\
\text { Waller, 2007) } \\
\text { Development of a } \\
\text { measure to assess } \\
\text { invalidating } \\
\text { childhood } \\
\text { environments in the } \\
\text { eating disorders }\end{array}$ & $\begin{array}{l}\text { Cross-sectional: One- } \\
\text { time survey collection. } \\
\text { Analysis by correlation } \\
\text { and t-tests. }\end{array}$ & $\begin{array}{l}\text { Emotional self-report: } \\
\text { cDTS } \\
++\mathrm{N}=63 \text {; Matched } \\
\text { undergraduate and } \\
\text { graduate females in } \\
\text { Britain without history } \\
\text { of an ED. } \\
\mathrm{N}=73 \text {; Female } \\
\text { inpatients with an ED. }\end{array}$ & $\begin{array}{l}\text { The clinical group scored higher on cDTS: } \\
\text { Avoidance and lower on cDTS: Accept and Manage } \\
\text { than the nonclinical group. The clinical and } \\
\text { nonclinical group did not differ on the cDTS: } \\
\text { Anticipate and Distract. cDTS: Avoidance was } \\
\text { associated with a variety of invalidating childhood } \\
\text { environments. cDTS: Avoidance partially mediated } \\
\text { the relationship between a paternal invalidating } \\
\text { environment and ED symptoms. }\end{array}$ \\
\hline $\begin{array}{l}\text { (Raykos et al., 2009) } \\
\text { Confirmatory and } \\
\text { exploratory factor } \\
\text { analysis of the } \\
\text { distress tolerance } \\
\text { scale (DTS) in a } \\
\text { clinical sample of } \\
\text { eating disorder } \\
\text { patients }\end{array}$ & $\begin{array}{l}\text { Cross-sectional: One } \\
\text { time survey collection. } \\
\text { Analysis by CFA, EFA, } \\
\text { and correlations. }\end{array}$ & $\begin{array}{l}\text { Emotional self-report: } \\
\text { cDTS } \\
\mathrm{N}=214 \text {; Outpatients } \\
\text { receiving treatment for } \\
\text { ED. }\end{array}$ & $\begin{array}{l}\text { cDTS: Behavioral Avoidance of Positive Affect was } \\
\text { positively correlated with global eating pathology, } \\
\text { restraint, eating concern, weight concern, and shape } \\
\text { concern. cDTS: Anticipating and Managing Affect } \\
\text { was positively correlated with weight concern. }\end{array}$ \\
\hline $\begin{array}{l}\text { (Schoenefeld \& } \\
\text { Webb, 2013) } \\
\text { Self-compassion and } \\
\text { intuitive eating in } \\
\text { college women: } \\
\text { Examining the } \\
\text { contributions of } \\
\text { distress tolerance and } \\
\text { body image } \\
\text { acceptance and action }\end{array}$ & $\begin{array}{l}\text { Cross-sectional: One- } \\
\text { time survey collection. } \\
\text { Analysis by correlation } \\
\text { and by a multiple } \\
\text { mediator model. }\end{array}$ & $\begin{array}{l}\text { Emotional self-report: } \\
\text { sDTS } \\
\mathrm{N}=322 \\
\text { Undergraduate } \\
\text { females. }\end{array}$ & $\begin{array}{l}\text { DT was positively correlated with intuitive eating, } \\
\text { body image acceptance, self-compassion, and self- } \\
\text { esteem. The role of DT as mediator between self- } \\
\text { compassion and intuitive eating was non-significant } \\
\text { once accounting for body image acceptance. }\end{array}$ \\
\hline
\end{tabular}




\begin{tabular}{|c|c|c|c|}
\hline \multicolumn{4}{|l|}{ NSSI } \\
\hline $\begin{array}{l}\text { (Anestis, Kleiman, et } \\
\text { al., 2014) } \\
\text { The pursuit of death } \\
\text { versus escape from } \\
\text { negative affect: An } \\
\text { examination of the } \\
\text { nature of the } \\
\text { relationship between } \\
\text { emotion } \\
\text { dysregulation and } \\
\text { both suicidal } \\
\text { behavior and non- } \\
\text { suicidal self-injury }\end{array}$ & $\begin{array}{l}\text { Cross-sectional: One- } \\
\text { time survey collection. } \\
\text { Analysis by correlation } \\
\text { and mediation analysis. }\end{array}$ & $\begin{array}{l}\text { Emotional self-report: } \\
\text { sDTS } \\
\text { Study 1: } \mathrm{N}=1317 \text {; } \\
\text { Undergraduates. }\end{array}$ & $\begin{array}{l}\text { DT was negatively correlated with emotion } \\
\text { dysregulation, NSSI frequency, and number of } \\
\text { suicide attempts. NSSI lifetime frequency partially } \\
\text { mediated the relationship between DT and lifetime } \\
\text { suicide attempts. However, the direct effect of DT } \\
\text { on lifetime suicide attempts remained significant. }\end{array}$ \\
\hline $\begin{array}{l}\text { (Anestis, Knorr, et } \\
\text { al., 2013) } \\
\text { The importance of } \\
\text { high distress } \\
\text { tolerance in the } \\
\text { relationship between } \\
\text { nonsuicidal self- } \\
\text { injury and suicide } \\
\text { potential }\end{array}$ & $\begin{array}{l}\text { Cross-sectional: One- } \\
\text { time survey collection. } \\
\text { Analysis by correlation } \\
\text { and hierarchical linear } \\
\text { regressions. }\end{array}$ & $\begin{array}{l}\text { Emotional self-report: } \\
\text { sDTS } \\
\mathrm{N}=93 \text {; Inpatients } \\
\text { receiving SUD } \\
\text { treatment. }\end{array}$ & $\begin{array}{l}\text { DT was negatively correlated with depression, NSSI } \\
\text { frequency, NSSI methods, and suicide potential. DT } \\
\text { interacted with NSSI frequency (but not NSSI } \\
\text { methods) in the prediction of suicide potential, after } \\
\text { controlling for sex, age, income, marital status, and } \\
\text { depression. As such, the magnitude of the } \\
\text { relationship between NSSI frequency and suicide } \\
\text { potential was greatest at high levels of DT. }\end{array}$ \\
\hline
\end{tabular}




\begin{tabular}{|c|c|c|c|}
\hline $\begin{array}{l}\text { (Anestis, Pennings, et } \\
\text { al., 2013) } \\
\text { Low distress } \\
\text { tolerance as an } \\
\text { indirect risk factor for } \\
\text { suicidal behavior: } \\
\text { Considering the } \\
\text { explanatory role of } \\
\text { non-suicidal self- } \\
\text { injury }\end{array}$ & $\begin{array}{l}\text { Cross-sectional: One- } \\
\text { time survey collection. } \\
\text { Analysis by correlation } \\
\text { and mediation analysis. }\end{array}$ & $\begin{array}{l}\text { Emotional self-report: } \\
\text { sDTS } \\
\mathrm{N}=93 \text {; Inpatients } \\
\text { receiving SUD } \\
\text { treatment. }\end{array}$ & $\begin{array}{l}\text { DT was negatively correlated with depression, NSSI } \\
\text { lifetime episodes, and suicidal behavior. NSSI } \\
\text { lifetime episode mediated the relationship between } \\
\text { low DT and lifetime suicide attempts, after } \\
\text { controlling for sex, income, age, and depression } \\
\text { symptoms. }\end{array}$ \\
\hline $\begin{array}{l}\text { (Booth et al., 2014) } \\
\text { Living through } \\
\text { distress: A skills } \\
\text { training group for } \\
\text { reducing deliberate } \\
\text { self-harm }\end{array}$ & $\begin{array}{l}\text { Experimental: } \\
\text { Assessments completed } \\
\text { pre-treatment, post- } \\
\text { treatment, and at } 3 \\
\text { months post-treatment. } \\
\text { Treatment involved } \\
\text { group therapy for one } \\
\text { hour a day, four days a } \\
\text { week, for six weeks. } \\
\text { Analysis by correlation } \\
\text { and ANOVA. }\end{array}$ & $\begin{array}{l}\text { Emotional self-report: } \\
\text { sDTS } \\
\mathrm{N}=114 \text {; Inpatients } \\
\text { with a history of NSSI } \\
\text { or suicidal ideation. }\end{array}$ & $\begin{array}{l}\text { DT decreased between pre-treatment and post- } \\
\text { treatment, and between pre-treatment and } 3 \text { months } \\
\text { post-treatment. NSSI also decreased between pre- } \\
\text { treatment and post-treatment, and between pre- } \\
\text { treatment and } 3 \text { months post-treatment. }\end{array}$ \\
\hline
\end{tabular}




\begin{tabular}{|c|c|c|c|}
\hline $\begin{array}{l}\text { (Gratz et al., 2011) } \\
\text { An experimental } \\
\text { investigation of } \\
\text { emotional willingness } \\
\text { and physical pain } \\
\text { tolerance in } \\
\text { deliberate self-harm: } \\
\text { the moderating role } \\
\text { of interpersonal } \\
\text { distress }\end{array}$ & $\begin{array}{l}\text { Cross-sectional: One- } \\
\text { time survey collection } \\
\text { and baseline cold } \\
\text { pressor task. Then, } \\
\text { either a neutral or } \\
\text { distressing } \\
\text { interpersonal script was } \\
\text { read. After the script, } \\
\text { the Algometer task was } \\
\text { administered. Negative } \\
\text { affect was assessed at } \\
\text { four different points } \\
\text { throughout. Analysis } \\
\text { by correlation, } t \text {-test, } \\
\text { and ANCOVA. }\end{array}$ & $\begin{array}{l}\text { Emotional behavioral: } \\
\text { MTPT } \\
\text { Physical behavioral: } \\
\text { Cold pressor and } \\
\text { Algometer } \\
\mathrm{N}=43 \text {; Nonclinical } \\
\text { individuals with at } \\
\text { least one NSSI } \\
\text { episode within the last } \\
\text { year. } \\
\mathrm{N}=52 \text {; Nonclinical } \\
\text { individuals without } \\
\text { NSSI history. }\end{array}$ & $\begin{array}{l}\text { MTPT latency to termination was not associated } \\
\text { with depression symptoms, BPD symptoms, lifetime } \\
\text { NSSI frequency, or negative affect. MTPT latency to } \\
\text { termination was correlated with pain tolerance on } \\
\text { cold pressor task, but not on the algometer task. } \\
\text { Women from the NSSI group in the distressing } \\
\text { condition terminated the MTPT faster than control } \\
\text { group. Whereas, women from the NSSI groups in } \\
\text { the neutral condition endured the MTPT longer than } \\
\text { the control group. However, the opposite pattern was } \\
\text { found for men. On the algometer task, individuals } \\
\text { from the NSSI group endured longer than the control } \\
\text { group. }\end{array}$ \\
\hline $\begin{array}{l}\text { (Peterson et al., 2014) } \\
\text { Interactive role of } \\
\text { depression, distress } \\
\text { tolerance and } \\
\text { negative urgency on } \\
\text { non-suicidal self- } \\
\text { injury }\end{array}$ & $\begin{array}{l}\text { Cross-sectional: One- } \\
\text { time survey collection. } \\
\text { Analysis by correlation, } \\
\text { regression analysis, and } \\
\text { logistic regression. }\end{array}$ & $\begin{array}{l}\text { Emotional self-report: } \\
\text { sDTS } \\
\mathrm{N}=884 \\
\text { Undergraduates. }\end{array}$ & $\begin{array}{l}\text { DT was negatively correlated with NSSI and } \\
\text { depression. DT did not have a main effect on NSSI. } \\
\text { However, there was a three way interaction such that } \\
\text { among individuals with low DT, high levels of } \\
\text { negative urgency and depression predicted higher } \\
\text { levels of NSSI. }\end{array}$ \\
\hline
\end{tabular}




\begin{tabular}{|c|c|c|c|}
\hline \multicolumn{4}{|l|}{ OVEREATING } \\
\hline $\begin{array}{l}\text { (Kelly et al., 2014) } \\
\text { Examining the role of } \\
\text { distress tolerance and } \\
\text { negative urgency in } \\
\text { binge eating behavior } \\
\text { among women }\end{array}$ & $\begin{array}{l}\text { Cross-sectional: One- } \\
\text { time survey collection. } \\
\text { Analysis by } \\
\text { hierarchical regression. }\end{array}$ & $\begin{array}{l}\text { Emotional self-report: } \\
\text { sDTS } \\
\mathrm{N}=18 \\
\text { Undergraduate } \\
\text { females reporting } \\
\text { binge eating behavior } \\
\text { in the last } 28 \text { days }\end{array}$ & $\begin{array}{l}\text { DT was negatively correlated with binge eating } \\
\text { episodes. However, there was no main effect of DT } \\
\text { on binge eating episodes after controlling for } \\
\text { race/ethnicity, BMI, and impulsivity. DT also did } \\
\text { not moderate the association between depression } \\
\text { symptoms and binge eating frequency or the } \\
\text { association between disordered eating attitudes and } \\
\text { binge eating frequency. }\end{array}$ \\
\hline $\begin{array}{l}\text { (Kenardy et al., 1996) } \\
\text { The aversiveness of } \\
\text { specific emotional } \\
\text { states associated with } \\
\text { binge-eating in obese } \\
\text { subjects }\end{array}$ & $\begin{array}{l}\text { Cross-sectional: One- } \\
\text { time survey collection. } \\
\text { Analysis by stepwise } \\
\text { discriminant function } \\
\text { analysis. }\end{array}$ & $\begin{array}{l}\text { Emotional self-report: } \\
\text { ETS } \\
\mathrm{N}=98 ; \text { Females } \\
\text { meeting criteria for } \\
\text { Binge-Eating Disorder } \\
\mathrm{N}=65 ; \text { Healthy } \\
\text { controls }\end{array}$ & $\begin{array}{l}\text { Females with Binge-Eating Disorder reported lower } \\
\text { emotional tolerance (more fear) in response to } \\
\text { emotions typically reported prior to binge eating } \\
\text { episodes than individuals in the control group did. }\end{array}$ \\
\hline $\begin{array}{l}\text { (Kozak \& Fought, } \\
\text { 2011) } \\
\text { Beyond alcohol and } \\
\text { drug addiction. Does } \\
\text { the negative trait of } \\
\text { low distress tolerance } \\
\text { have an association } \\
\text { with overeating? }\end{array}$ & $\begin{array}{l}\text { Cross-sectional: One- } \\
\text { time survey collection. } \\
\text { Analysis by correlation } \\
\text { and linear regression } \\
\text { analysis. }\end{array}$ & $\begin{array}{l}\text { Emotional self-report: } \\
\text { sDTS } \\
\mathrm{N}=225 \\
\text { Undergraduates. }\end{array}$ & $\begin{array}{l}\text { Low DT significantly predicted emotional eating, } \\
\text { external eating, and disinhibition, after controlling } \\
\text { for sex and BMI. }\end{array}$ \\
\hline
\end{tabular}




\begin{tabular}{|c|c|c|c|}
\hline $\begin{array}{l}\text { (Webb \& Forman, } \\
2013 \text { ) } \\
\text { Evaluating the } \\
\text { indirect effect of self- } \\
\text { compassion on binge } \\
\text { eating severity } \\
\text { through cognitive- } \\
\text { affective self- } \\
\text { regulatory pathways }\end{array}$ & $\begin{array}{l}\text { Cross-sectional: One- } \\
\text { time survey collection. } \\
\text { Analysis in a single } \\
\text { multiple mediator } \\
\text { model. }\end{array}$ & $\begin{array}{l}\text { Emotional self-report: } \\
\text { ETS } \\
\mathrm{N}=215 \\
\text { Undergraduates. }\end{array}$ & $\begin{array}{l}\text { The relationship between positive-self compassion } \\
\text { and binge eating severity was mediated by both } \\
\text { emotional tolerance and unconditional self- } \\
\text { acceptance, after controlling for BMI. }\end{array}$ \\
\hline \multicolumn{4}{|l|}{ SUICIDE } \\
\hline $\begin{array}{l}\text { (Anestis, Bagge, et } \\
\text { al., 2011) } \\
\text { Clarifying the role of } \\
\text { emotion } \\
\text { dysregulation in the } \\
\text { interpersonal- } \\
\text { psychological theory } \\
\text { of suicidal behavior } \\
\text { in an undergraduate } \\
\text { sample }\end{array}$ & $\begin{array}{l}\text { Cross-sectional: One- } \\
\text { time survey collection } \\
\text { followed by behavioral } \\
\text { task. Analysis by } \\
\text { correlation and } \\
\text { hierarchical linear } \\
\text { regressions. }\end{array}$ & $\begin{array}{l}\text { Emotional self-report: } \\
\text { sDTS } \\
\text { Physical behavioral: } \\
\text { Pressure Algometer } \\
++++\mathrm{N}=283 \text {; Right- } \\
\text { handed } \\
\text { undergraduates. }\end{array}$ & $\begin{array}{l}\text { Lower levels of DT predicted increased perceived } \\
\text { burdensomeness, thwarted belongingness, and pain } \\
\text { tolerance, after controlling for sensation seeking, } \\
\text { lack of premeditation, lack of perseverance, } \\
\text { depression, anxiety, and sex. DT did not predict } \\
\text { acquired capability for suicide, after controlling for } \\
\text { the same variables. }\end{array}$ \\
\hline $\begin{array}{l}\text { (Anestis, Bender, et } \\
\text { al., 2011) } \\
\text { Sex and emotion in } \\
\text { the acquired } \\
\text { capability for suicide }\end{array}$ & $\begin{array}{l}\text { Cross-sectional: One- } \\
\text { time survey collection. } \\
\text { Analysis by correlation } \\
\text { and hierarchical linear } \\
\text { regressions. }\end{array}$ & $\begin{array}{l}\text { Emotional self-report: } \\
\text { sDTS } \\
+++++\mathrm{N}=200 \\
\text { Undergraduates. }\end{array}$ & $\begin{array}{l}\text { DT was positively correlated with acquired } \\
\text { capability for suicide. DT had a direct effect on } \\
\text { acquired capability for suicide, while accounting for } \\
\text { sensation seeking. DT and sex also interacted in the } \\
\text { prediction of acquired capability for suicide: men } \\
\text { with high DT had the highest acquired capability for } \\
\text { suicide. Further, DT predicted acquired capability in } \\
\text { men, but not in women. }\end{array}$ \\
\hline
\end{tabular}




\begin{tabular}{|c|c|c|c|}
\hline $\begin{array}{l}\text { (Anestis \& Joiner, } \\
\text { 2012) } \\
\text { Behaviorally-indexed } \\
\text { distress tolerance and } \\
\text { suicidality }\end{array}$ & $\begin{array}{l}\text { Cross-sectional: One- } \\
\text { time survey collection } \\
\text { and behavioral task. } \\
\text { Except negative affect } \\
\text { was assessed twice } \\
\text { throughout task. } \\
\text { Analysis by correlation } \\
\text { and hierarchical linear } \\
\text { regression. }\end{array}$ & $\begin{array}{l}\text { Emotional behavioral: } \\
\text { DTT } \\
++++\mathrm{N}=283 \text {; Right- } \\
\text { handed } \\
\text { undergraduates. }\end{array}$ & $\begin{array}{l}\text { Behavioral DT was not correlated with perceived } \\
\text { burdensomeness and thwarted belongingness. DT } \\
\text { was positively correlated with acquired capability } \\
\text { for suicide and negatively correlated with the } \\
\text { lifetime number of painful/provocative experiences. } \\
\text { DT significantly predicted acquired capability for } \\
\text { suicide, such that higher levels of DT were } \\
\text { associated with higher acquired capability for } \\
\text { suicide, after accounting for sex and number of } \\
\text { painful/provocative events. DT also interacted with } \\
\text { painful/provocative experiences in the prediction of } \\
\text { acquired capability for suicide, such that the } \\
\text { predictive power of painful/provocative events is } \\
\text { greater at higher levels of DT. }\end{array}$ \\
\hline $\begin{array}{l}\text { (Anestis, Moberg, et } \\
\text { al., 2014) } \\
\text { Hope and the } \\
\text { interpersonal- } \\
\text { psychological theory } \\
\text { of suicidal behavior: } \\
\text { Replication and } \\
\text { extension of prior } \\
\text { findings }\end{array}$ & $\begin{array}{l}\text { Cross-sectional: One- } \\
\text { time online survey } \\
\text { collection. Analysis by } \\
\text { correlation, hierarchical } \\
\text { multiple regression, } \\
\text { and mediation models. }\end{array}$ & $\begin{array}{l}\text { Emotional self-report: } \\
\text { sDTS } \\
\mathrm{N}=220 \\
\text { Undergraduates. }\end{array}$ & $\begin{array}{l}\text { DT was negatively correlated with depression, } \\
\text { perceived burdensomeness, and thwarted } \\
\text { belongingness, but positively correlated with } \\
\text { acquired capability for suicide, and trait hope. DT } \\
\text { mediated the predictive relationship between trait } \\
\text { hope and acquired capability for suicide, after } \\
\text { controlling for sex, age, income, and } \\
\text { painful/provocative events. DT had a direct effect on } \\
\text { acquired capability for suicide, and the effect was } \\
\text { not mediated by trait hope. }\end{array}$ \\
\hline
\end{tabular}




\begin{tabular}{|c|c|c|c|}
\hline \multirow{2}{*}{$\begin{array}{l}\text { (Bender et al., 2012) } \\
\text { Affective and } \\
\text { behavioral paths } \\
\text { toward the acquired } \\
\text { capacity for suicide }\end{array}$} & $\begin{array}{l}\text { Study 1: Cross- } \\
\text { sectional: One-time } \\
\text { survey collection. } \\
\text { Analysis by correlation } \\
\text { and hierarchical linear } \\
\text { regression. }\end{array}$ & $\begin{array}{l}\text { Emotional self-report: } \\
\text { sDTS } \\
+++++\mathrm{N}=200 \\
\text { Undergraduates. }\end{array}$ & $\begin{array}{l}\text { DTS was negatively correlated with depression, } \\
\text { anxiety, and negative urgency and positively } \\
\text { correlated with lack of perseverance and acquired } \\
\text { capability for suicide. DT and sensation seeking } \\
\text { interacted to predict acquired capability for suicide } \\
\text { with the following covariates: sex, negative urgency, } \\
\text { lack of premeditation, lack of perseverance, } \\
\text { depression, anxiety, AS, and perfectionism. }\end{array}$ \\
\hline & $\begin{array}{l}\text { Study 2: Cross- } \\
\text { sectional: One-time } \\
\text { survey collection } \\
\text { followed by behavioral } \\
\text { task. Analysis by } \\
\text { correlation and } \\
\text { hierarchical linear } \\
\text { regression. }\end{array}$ & $\begin{array}{l}\text { Emotional behavioral: } \\
\text { DTT } \\
\text { Physical behavioral: } \\
\text { Pressure algometer } \\
++++\mathrm{N}=283 \text {; Right- } \\
\text { handed } \\
\text { undergraduates. }\end{array}$ & $\begin{array}{l}\text { DT was not correlated with sex, negative urgency, } \\
\text { lack of premeditation, lack of perseverance, } \\
\text { depression, anxiety, perfectionism, } \\
\text { painful/provocative events, physical pain tolerance, } \\
\text { or sensation seeking. DT and sensation seeking } \\
\text { interacted to predict pain tolerance, after covarying } \\
\text { for sex, negative urgency, lack of premeditation, } \\
\text { lack of perseverance, depression, anxiety, } \\
\text { perfectionism, and painful/provocative events. } \\
\text { Individuals with high DT and high sensation seeking } \\
\text { had the highest pain tolerance. }\end{array}$ \\
\hline
\end{tabular}




\begin{tabular}{|c|c|c|c|}
\hline $\begin{array}{l}\text { (Capron et al., 2013) } \\
\text { Distress tolerance } \\
\text { and anxiety } \\
\text { sensitivity cognitive } \\
\text { concerns: testing the } \\
\text { incremental } \\
\text { contributions of } \\
\text { affect dysregulation } \\
\text { constructs on suicidal } \\
\text { ideation and suicide } \\
\text { attempt }\end{array}$ & $\begin{array}{l}\text { Cross-sectional: One- } \\
\text { time survey collection. } \\
\text { Analysis by correlation } \\
\text { and multiple } \\
\text { regression. }\end{array}$ & $\begin{array}{l}\text { Emotional self-report: } \\
\text { sDTS } \\
\mathrm{N}=192 \text {; Outpatients } \\
\text { receiving services at } \\
\text { university clinic. }\end{array}$ & $\begin{array}{l}\text { DT was negatively correlated with AS, depression, } \\
\text { and suicidal ideation. DT did not predict suicidal } \\
\text { ideation after controlling for depression, sex, and } \\
\text { AS, although the relationship trended toward } \\
\text { significance. DT did not predict past suicide attempt } \\
\text { with the same controls. }\end{array}$ \\
\hline
\end{tabular}

BHD= Breath Holding Duration task (Hajek, Belcher, \& Stapleton, 1987); cDTS= Distress Tolerance Scale (Corstorphine et స్ al., 2007); DII= Distress Intolerance Index (McHugh \& Otto, 2012); DIS= Discomfort Intolerance Scale (Schmidt et al., 2006); DTT= Distress Tolerance Test (Nock \& Mendes, 2008); sDTS= Distress Tolerance Scale (Simons \& Gaher, 2005); ETS=

Emotional Tolerance Scale (Kenardy et al., 1996); MTPT= Mirror-tracing Persistence Task (Quinn et al., 1996) 
APPENDIX B

APPROVAL LETTERS 


\section{Andrews $\mathbf{Q}$ University}

June 28, 2016

Brittany Sommers (PI)

Tel: (330) 388-5079

Email: sommersb@andrews.edu

Tevni Grajales (CoPI): Tel. 269-471-3476; tevni@andrews.edu

Bradly Hinman (CoPI): Tel. 269-471-3466; hibradly@andrews.edu

RE: APPLICATION FOR APPROVAL OF RESEARCH INVOLVING HUMAN SUBJECTS IRB Protocol \#: 16-077 Application Type: Original Dept.: Graduate Psychology \& Counseling Review Category: Full Action Taken: Approved Advisor: Ronald Coffen

Title: A model of distress tolerance in self-damaging behaviors: Examining the role of emotional reactivity and learned helplessness

This letter is to advise you that the Institutional Review Board (IRB) has reviewed and approved your IRB application for research involving human subjects entitled: "A model of distress tolerance in self-damaging behaviors: Examining the role of emotional reactivity and learned helplessness " IRB protocol number 16-077 under Full category. This approval is valid until June 28, 2017. If your research is not completed by the end of this period you must apply for an extension at least four weeks prior to the expiration date. We ask that you inform IRB Office whenever you complete your research. Please reference the protocol number in future correspondence regarding this study.

Any future changes (see IRB Handbook pages 10-11) made to the study design and/or consent form require prior approval from the IRB before such changes can be implemented. To request for extension, modification and completion of your study please use the attached form.

While there appears to be no more than minimum risk with your study, should an incidence occur that results in a research-related adverse reaction and/or physical injury, (see IRB Handbook page 11) this must be reported immediately in writing to the IRB. Any project-related physical injury must also be reported immediately to the University physician, Dr. Reichert, by calling (269) 473-2222.

We wish you success in your research project. Please feel free to contact our office if you have questions.

Sincerely,

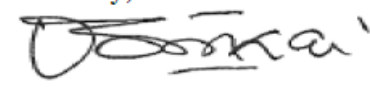

Mordekai Ongo

Research Integrity \& Compliance Officer

Institutional Review Board - 4150 Administration Dr Room 322 - Berrien Springs, MI 49104-0355

Tel: (269) 471-6361 Fax: (269) 471-6543 E-mail: irb@andrews.edu 


\section{P QuestionPro}

\section{Online Research Made Easy}

Date: $06 / 03 / 2016$

\section{Re: Permission to Conduct Research Using QuestionPro}

To,

Institutional Review Board

Andrews University

4150 Administrative Drive,

Room 322 Berrien Springs,

MI 49104-0355

This letter is being produced in response to a request by Brittany Sommers(sommersb@andrews.edu), a student at your institution who wishes to conduct a survey using the QuestionPro tool and collect responses through the QuestionPro panel in order to support her research titled 'A model of distress tolerance in self-damaging behaviors: Examining the role of emotional reactivity and learned helplessness'. The student has indicated that she requires a letter from QuestionPro granting her permission to do this. Please accept this letter as evidence of such permission. Students are permitted to conduct research via the QuestionPro platform provided that they abide by our Terms of Use, a copy of which is available on our website at:

http://www.questionpro.com/help/2.html

QuestionPro is a self-serve survey platform on which our users can, by themselves, create, deploy and analyze surveys through an online interface. We have users in many different industries that use surveys for many different purposes. One of our most common use cases is students and other types of researchers using our online tools to conduct academic research.

If you have any questions about this letter, please contact us at the email address below.

Sincerely,

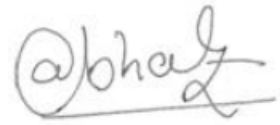

Aditya Bhat (Director of Sales)

QuestionPro Inc. 
APPENDIX C

INFORMED CONSENT 


\section{INFORMED CONSENT}

\section{Purpose}

You are invited to participate in a research project titled A Model of Distress Tolerance in Self-damaging Behaviors: Examining the Role of Emotional Reactivity and Learned Helplessness. The purpose of this research is to determine if certain personal characteristics are related to one's ability to tolerate unpleasant emotions.

\section{Researchers}

This research is being conducted by Brittany Sommers, a doctoral student in the department of Graduate Psychology and Counseling at Andrews University in Berrien Springs, Michigan. The research is being supervised by Dr. Ron Coffen, PhD, LP. Results from this research will be used in Brittany Sommers's dissertation and may be published in professional journals and/or presented at conferences without any information that could identify you.

\section{Procedure}

If you choose to participate in this research, you will be asked to complete a survey that asks questions about your demographics, emotions, and behaviors. It will take approximately 15 minutes to complete the survey.

\section{Participation}

In order to participate, you must be over 18 years of age and reside in the USA. You must also have a lifetime history of having experienced at least one of the following: binge eating, purging, restricted eating, nonsuicidal self-injury, suicidal ideation, or a suicide attempt. Your participation in this study is completely voluntary. It is your choice whether to participate or not. You may quit the survey at any time.

\section{Risks, Benefits, and Compensation}

This study addresses sensitive topics including suicidality. Thinking about sensitive topics may trigger upsetting thoughts or emotions. If taking the survey elicits thoughts of harming yourself, please go to the emergency room or contact one of the following numbers:

911

1-800-273-TALK (1-800-273-8255)
For emergency services (in the USA) To reach a trained counselor

If you desire to speak with a mental health professional (non-emergency) after taking the survey, please go to https://therapists.psychologytoday.com/rms/ or call 211 to find a mental health professional near you.

By completing this survey, you may contribute to a better understanding of personal characteristics which contribute to an individual's ability to tolerate unpleasant emotions. As with all surveys taken through this company, if you choose to complete this survey you will be awarded points by QuestionPro which are later redeemable for prizes. 


\section{Confidentiality}

Your survey responses will be strictly confidential and data from this research will be reported only in the aggregate. Your information will be coded and will remain confidential.

\section{Contact Information}

If you have questions at any time about the survey, your participation in this research, or your rights as a participant, you may contact the principle investigator, Brittany Sommers, at (330) 330-5079 or sommersb@andrews.edu. You may also contact her research advisor, Dr. Ron Coffen, at (269) 471-3491 or coffen@ andrews.edu. This study has been approved by the Institutional Review Board at Andrews University (\#16-077), who can be reached at (269) 471-6361 or irb@ andrews.edu.

\section{Consent}

Thank you very much for your time and support! Please start the survey by clicking on the Continue button below. By clicking the button you are giving your consent to participate in the research described above. 
APPENDIX D

DEMOGRAPHIC QUESTIONNAIRE 


\section{DEMOGRAPHIC QUESTIONNAIRE}

What is your age?

What is your sex?

- Male

- Female

- Intersex

o Other

What is your marital status?

○ Single, never married

- Engaged or Married

- Separated or Divorced

- Widowed

Which of the following ethnic groups do you most identify with?
- African American
- Latino/a American
- American Indian
- Asian American
- Caucasian American
- Multiracial
○ Other

How much total combined money did your household make last year?
○ $\$ 0$ to $\$ 9,999$
○ $\$ 10,000$ to $\$ 24,999$
○ $\$ 25,000$ to $\$ 49,999$
○ $\$ 50,000$ to $\$ 74,999$
- $\$ 75,000$ to $\$ 99,999$
- $\$ 100,000$ to $\$ 124,999$
- $\$ 125,000$ to $\$ 149,999$
- $\$ 150,000$ to $\$ 174,999$
- $\$ 175,000$ to $\$ 199,999$
○ $\$ 200,000$ and up 
APPENDIX E

SURVEYS 


\section{EMOTION REACTIVITY SCALE}

This questionnaire asks different questions about how you experience emotions on a regular basis (for example, each day). When you are asked about being "emotional," this may refer to being angry, sad, excited, or some other emotion. Please rate the following statements.

$0=$ Not at all like me

$1=$ A little like me

$2=$ Somewhat like me

$3=$ A lot like me

4 = Completely like me

1. When something happens that upsets me, it's all I can think about for $\quad \begin{array}{llllll}0 & 1 & 2 & 3 & 4\end{array}$ a long time.

\begin{tabular}{llllll}
\hline $\begin{array}{l}\text { 2. My feelings get hurt easily. } \\
\text { 3. When I experience emotions, I feel } \\
\text { them very strongly/intensely. }\end{array}$ & 0 & 1 & 2 & 3 & 4 \\
\hline $\begin{array}{l}\text { 4. When I'm emotionally upset, my } \\
\text { whole body gets physically upset as } \\
\text { well. }\end{array}$ & 0 & 1 & 2 & 3 & 4 \\
\hline $\begin{array}{l}\text { 5. I tend to get very emotional very } \\
\text { easily. }\end{array}$ & 0 & 1 & 2 & 3 & 4 \\
\hline $\begin{array}{l}\text { 6. I experience emotions very } \\
\text { strongly. }\end{array}$ & 0 & 1 & 2 & 3 & 4 \\
\hline $\begin{array}{l}\text { 7. I often feel extremely anxious. } \\
\text { 8. When I feel emotional, it's hard for } \\
\text { me to imagine feeling any other way. }\end{array}$ & 0 & 1 & 2 & 3 & 4 \\
\hline $\begin{array}{l}\text { 9. Even the littlest things make me } \\
\text { emotional. }\end{array}$ & 0 & 1 & 2 & 3 & 3 \\
$\begin{array}{l}\text { 10. If I have a disagreement with } \\
\text { someone, it takes a long time for me } \\
\text { to get over it. }\end{array}$ & 0 & 1 & 2 & 3 & 4 \\
\hline
\end{tabular}




\begin{tabular}{|c|c|c|c|c|c|}
\hline $\begin{array}{l}\text { 11. When I am angry/upset, it takes } \\
\text { me much longer than most people to } \\
\text { calm down. }\end{array}$ & 0 & 1 & 2 & 3 & 4 \\
\hline 12. I get angry at people very easily. & 0 & 1 & 2 & 3 & 4 \\
\hline $\begin{array}{l}\text { 13. I am often bothered by things that } \\
\text { other people don't react to. }\end{array}$ & 0 & 1 & 2 & 3 & 4 \\
\hline 14. I am easily agitated. & 0 & 1 & 2 & 3 & 4 \\
\hline $\begin{array}{l}\text { 15. My emotions go from neutral to } \\
\text { extreme in an instant. }\end{array}$ & 0 & 1 & 2 & 3 & 4 \\
\hline $\begin{array}{l}\text { 16. When something bad happens, my } \\
\text { mood changes very quickly. People } \\
\text { tell me I have a very short fuse. }\end{array}$ & 0 & 1 & 2 & 3 & 4 \\
\hline $\begin{array}{l}\text { 17. People tell me that my emotions } \\
\text { are often too intense for the situation. }\end{array}$ & 0 & 1 & 2 & 3 & 4 \\
\hline 18. I am a very sensitive person. & 0 & 1 & 2 & 3 & 4 \\
\hline $\begin{array}{l}\text { 19. My moods are very strong and } \\
\text { powerful. }\end{array}$ & 0 & 1 & 2 & 3 & 4 \\
\hline $\begin{array}{l}20 . \text { I often get so upset it's hard for me } \\
\text { to think straight. }\end{array}$ & 0 & 1 & 2 & 3 & 4 \\
\hline $\begin{array}{l}\text { 21. Other people tell me I'm } \\
\text { overreacting. }\end{array}$ & 0 & 1 & 2 & 3 & 4 \\
\hline
\end{tabular}




\section{LEARNED HELPLESSNESS SCALE}

Please select the answer that most closely describes you or your feelings about yourself.

\begin{tabular}{|c|c|c|c|c|}
\hline $\begin{array}{l}\text { 1. No matter how much energy I } \\
\text { put into a task, I feel I have no } \\
\text { control over the outcome. }\end{array}$ & $\begin{array}{l}\text { Strongly } \\
\text { Agree }\end{array}$ & Agree & Disagree & $\begin{array}{l}\text { Strongly } \\
\text { Disagree }\end{array}$ \\
\hline $\begin{array}{l}\text { 2. I feel that my ability to solve } \\
\text { problems is the cause of my } \\
\text { success. }\end{array}$ & $\begin{array}{l}\text { Strongly } \\
\text { Agree }\end{array}$ & Agree & Disagree & $\begin{array}{l}\text { Strongly } \\
\text { Disagree }\end{array}$ \\
\hline $\begin{array}{l}\text { 3. I can find solutions to difficult } \\
\text { problems. }\end{array}$ & $\begin{array}{l}\text { Strongly } \\
\text { Agree }\end{array}$ & Agree & Disagree & $\begin{array}{l}\text { Strongly } \\
\text { Disagree }\end{array}$ \\
\hline $\begin{array}{l}\text { 4. I don't place myself in situations } \\
\text { in which I cannot predict the } \\
\text { outcome. }\end{array}$ & $\begin{array}{l}\text { Strongly } \\
\text { Agree }\end{array}$ & Agree & Disagree & $\begin{array}{l}\text { Strongly } \\
\text { Disagree }\end{array}$ \\
\hline $\begin{array}{l}\text { 5. If I complete a task successfully, } \\
\text { it is probably because of my ability. }\end{array}$ & $\begin{array}{l}\text { Strongly } \\
\text { Agree }\end{array}$ & Agree & Disagree & $\begin{array}{l}\text { Strongly } \\
\text { Disagree }\end{array}$ \\
\hline $\begin{array}{l}\text { 6. I have the ability to solve most of } \\
\text { life's problems. }\end{array}$ & $\begin{array}{l}\text { Strongly } \\
\text { Agree }\end{array}$ & Agree & Disagree & $\begin{array}{l}\text { Strongly } \\
\text { Disagree }\end{array}$ \\
\hline $\begin{array}{l}\text { 7. When I do not succeed at a task, } \\
\text { I do not attempt any similar tasks } \\
\text { because I feel that I would fail them } \\
\text { also. }\end{array}$ & $\begin{array}{l}\text { Strongly } \\
\text { Agree }\end{array}$ & Agree & Disagree & $\begin{array}{l}\text { Strongly } \\
\text { Disagree }\end{array}$ \\
\hline $\begin{array}{l}\text { 8. When something doesn't turn out } \\
\text { the way I planned, I know it is } \\
\text { because I didn't have the ability to } \\
\text { start with. }\end{array}$ & $\begin{array}{l}\text { Strongly } \\
\text { Agree }\end{array}$ & Agree & Disagree & $\begin{array}{l}\text { Strongly } \\
\text { Disagree }\end{array}$ \\
\hline $\begin{array}{l}\text { 9. Other people have more control } \\
\text { over their success and/or failure } \\
\text { than I do. }\end{array}$ & $\begin{array}{l}\text { Strongly } \\
\text { Agree }\end{array}$ & Agree & Disagree & $\begin{array}{l}\text { Strongly } \\
\text { Disagree }\end{array}$ \\
\hline $\begin{array}{l}\text { 10. I try new tasks if I have failed } \\
\text { similar ones in the past. }\end{array}$ & $\begin{array}{l}\text { Strongly } \\
\text { Agree }\end{array}$ & Agree & Disagree & $\begin{array}{l}\text { Strongly } \\
\text { Disagree }\end{array}$ \\
\hline $\begin{array}{l}\text { 11. When I perform poorly, it is } \\
\text { because I don't have the ability to } \\
\text { perform better. }\end{array}$ & $\begin{array}{l}\text { Strongly } \\
\text { Agree }\end{array}$ & Agree & Disagree & $\begin{array}{l}\text { Strongly } \\
\text { Disagree }\end{array}$ \\
\hline
\end{tabular}




\begin{tabular}{|c|c|c|c|c|}
\hline $\begin{array}{l}\text { 12. I accept tasks even if I am not } \\
\text { sure that I will succeed at them. }\end{array}$ & $\begin{array}{l}\text { Strongly } \\
\text { Agree }\end{array}$ & Agree & Disagree & $\begin{array}{l}\text { Strongly } \\
\text { Disagree }\end{array}$ \\
\hline $\begin{array}{l}\text { 13. I feel that I have little control } \\
\text { over the outcomes of my work. }\end{array}$ & $\begin{array}{l}\text { Strongly } \\
\text { Agree }\end{array}$ & Agree & Disagree & $\begin{array}{l}\text { Strongly } \\
\text { Disagree }\end{array}$ \\
\hline $\begin{array}{l}\text { 14. I am successful at most tasks I } \\
\text { try. }\end{array}$ & $\begin{array}{l}\text { Strongly } \\
\text { Agree }\end{array}$ & Agree & Disagree & $\begin{array}{l}\text { Strongly } \\
\text { Disagree }\end{array}$ \\
\hline $\begin{array}{l}\text { 15. I feel that anyone else could be } \\
\text { better than me at most tasks. }\end{array}$ & $\begin{array}{l}\text { Strongly } \\
\text { Agree }\end{array}$ & Agree & Disagree & $\begin{array}{l}\text { Strongly } \\
\text { Disagree }\end{array}$ \\
\hline $\begin{array}{l}\text { 16. I am able to reach my goals in } \\
\text { life. }\end{array}$ & $\begin{array}{l}\text { Strongly } \\
\text { Agree }\end{array}$ & Agree & Disagree & $\begin{array}{l}\text { Strongly } \\
\text { Disagree }\end{array}$ \\
\hline $\begin{array}{l}\text { 17. When I don't succeed at a task, } \\
\text { I find myself blaming my own } \\
\text { stupidity for my failure. }\end{array}$ & $\begin{array}{l}\text { Strongly } \\
\text { Agree }\end{array}$ & Agree & Disagree & $\begin{array}{l}\text { Strongly } \\
\text { Disagree }\end{array}$ \\
\hline $\begin{array}{l}\text { 18. No matter how hard I try, things } \\
\text { never seem to work out the way I } \\
\text { want them to. }\end{array}$ & $\begin{array}{l}\text { Strongly } \\
\text { Agree }\end{array}$ & Agree & Disagree & $\begin{array}{l}\text { Strongly } \\
\text { Disagree }\end{array}$ \\
\hline $\begin{array}{l}\text { 19. I feel that my success reflects } \\
\text { my ability, not chance. }\end{array}$ & $\begin{array}{l}\text { Strongly } \\
\text { Agree }\end{array}$ & Agree & Disagree & $\begin{array}{l}\text { Strongly } \\
\text { Disagree }\end{array}$ \\
\hline $\begin{array}{l}\text { 20. My behavior seems to influence } \\
\text { the success of a work group. }\end{array}$ & $\begin{array}{l}\text { Strongly } \\
\text { Agree }\end{array}$ & Agree & Disagree & $\begin{array}{l}\text { Strongly } \\
\text { Disagree }\end{array}$ \\
\hline
\end{tabular}




\section{DISTRESS TOLERANCE SCALE}

Think of times that you feel distressed or upset. Select the select the number that best describes your beliefs about feeling distressed or upset.

$1=$ Strongly agree

$2=$ Mildly agree

$3=$ Agree and disagree equally

$4=$ Mildly disagree

$5=$ Strongly disagree

\begin{tabular}{|c|c|c|c|c|c|}
\hline $\begin{array}{l}\text { 1. Feeling distressed or upset is } \\
\text { unbearable to me. }\end{array}$ & 1 & 2 & 3 & 4 & 5 \\
\hline $\begin{array}{l}\text { 2. When I feel distressed or upset, all I } \\
\text { can think about is how bad I feel. }\end{array}$ & 1 & 2 & 3 & 4 & 5 \\
\hline $\begin{array}{l}\text { 3. I can't handle feeling distressed or } \\
\text { upset. }\end{array}$ & 1 & 2 & 3 & 4 & 5 \\
\hline $\begin{array}{l}\text { 4. My feelings of distress are so } \\
\text { intense that they completely take over. }\end{array}$ & 1 & 2 & 3 & 4 & 5 \\
\hline $\begin{array}{l}\text { 5. There's nothing worse than feeling } \\
\text { distressed or upset. }\end{array}$ & 1 & 2 & 3 & 4 & 5 \\
\hline $\begin{array}{l}\text { 6. I can tolerate being distressed or } \\
\text { upset as well as most people. }\end{array}$ & 1 & 2 & 3 & 4 & 5 \\
\hline $\begin{array}{l}\text { 7. My feelings of distress or being } \\
\text { upset are not acceptable. }\end{array}$ & 1 & 2 & 3 & 4 & 5 \\
\hline $\begin{array}{l}\text { 8. I'll do anything to avoid feeling } \\
\text { distressed or upset. }\end{array}$ & 1 & 2 & 3 & 4 & 5 \\
\hline $\begin{array}{l}\text { 9. Other people seem to be able to } \\
\text { tolerate feeling distressed or upset } \\
\text { better than I can. }\end{array}$ & 1 & 2 & 3 & 4 & 5 \\
\hline $\begin{array}{l}\text { 10. Being distressed or upset is always } \\
\text { a major ordeal for me. }\end{array}$ & 1 & 2 & 3 & 4 & 5 \\
\hline $\begin{array}{l}\text { 11. I am ashamed of myself when I } \\
\text { feel distressed or upset. }\end{array}$ & 1 & 2 & 3 & 4 & 5 \\
\hline
\end{tabular}




\begin{tabular}{|c|c|c|c|c|c|}
\hline $\begin{array}{l}\text { 12. My feelings of distress or being } \\
\text { upset scare me. }\end{array}$ & 1 & 2 & 3 & 4 & 5 \\
\hline $\begin{array}{l}\text { 13. I'll do anything to stop feeling } \\
\text { distressed or upset. }\end{array}$ & 1 & 2 & 3 & 4 & 5 \\
\hline $\begin{array}{l}\text { 14. When I feel distressed or upset, I } \\
\text { must do something about it } \\
\text { immediately. }\end{array}$ & 1 & 2 & 3 & 4 & 5 \\
\hline $\begin{array}{l}\text { 15. When I feel distressed or upset, I } \\
\text { cannot help but concentrate on how } \\
\text { bad the distress actually feels. }\end{array}$ & 1 & 2 & 3 & 4 & 5 \\
\hline
\end{tabular}




\section{DEPRESSION ANXIETY STRESS SCALES 21}

\section{(Anxiety Subscale)}

Please read each statement and select a number $0,1,2$ or 3 that indicates how much the statement applied to you over the past week. There are no right or wrong answers. Do not spend too much time on any statement.

$0=$ Did not apply to me at all

$1=$ Applied to me to some degree, or some of the time

$2=$ Applied to me to a considerable degree, or a good part of the time

3 = Applied to me very much, or most of the time

\begin{tabular}{llll} 
1. I was aware of dryness of my mouth. & 0 & 1 & 2 \\
\hline $\begin{array}{l}\text { 2. I experienced breathing difficulty (e.g., } \\
\text { excessively rapid breathing, breathlessness in the } \\
\text { absence of physical exertion). }\end{array}$ & 0 & 1 & 2
\end{tabular}

\begin{tabular}{|c|c|c|c|c|}
\hline 3. I experienced trembling (e.g., in the hands). & 0 & 1 & 2 & 3 \\
\hline $\begin{array}{l}\text { 4. I was worried about situations in which I might } \\
\text { panic and make a fool of myself. }\end{array}$ & 0 & 1 & 2 & 3 \\
\hline 5. I felt I was close to panic. & 0 & 1 & 2 & 3 \\
\hline $\begin{array}{l}\text { 6. I was aware of the action of my heart in the } \\
\text { absence of physical exertion (e.g., sense of heart rate } \\
\text { increase, heart missing a beat). }\end{array}$ & 0 & 1 & 2 & 3 \\
\hline 7. I felt scared without any good reason. & 0 & 1 & 2 & 3 \\
\hline
\end{tabular}




\section{DEPRESSION ANXIETY STRESS SCALES 21}

\section{(Depression Subscale)}

Please read each statement and select a number $0,1,2$ or 3 that indicates how much the statement applied to you over the past week. There are no right or wrong answers. Do not spend too much time on any statement.

$0=$ Did not apply to me at all

$1=$ Applied to me to some degree, or some of the time

$2=$ Applied to me to a considerable degree, or a good part of the time

$3=$ Applied to me very much, or most of the time

\begin{tabular}{lllll}
\hline $\begin{array}{l}\text { 1. I couldn't seem to experience any positive feeling } \\
\text { at all. }\end{array}$ & 0 & 1 & 2 & 3 \\
\hline $\begin{array}{l}\text { 2. I found it difficult to work up the initiative to do } \\
\text { things. }\end{array}$ & 0 & 1 & 2 & 3 \\
\hline 3. I felt that I had nothing to look forward to. & 0 & 1 & 2 & 3 \\
\hline 4. I felt down-hearted and blue. & 0 & 1 & 2 & 3 \\
\hline $\begin{array}{l}\text { 5. I was unable to become enthusiastic about } \\
\text { anything. }\end{array}$ & 0 & 1 & 2 & 3 \\
\hline 6. I felt I wasn't worth much as a person. & 0 & 1 & 2 & 3 \\
\hline 7. I felt that life was meaningless. & 0 & 1 & 2 & 3 \\
\hline
\end{tabular}




\section{SELF-DAMAGING BEHAVIORS QUESTIONNAIRE}

(Adapted from the Self-Injurious Thoughts and Behavior Scale, Long form and the

$$
\text { Eating Disorder Exam Questionnaire 6.0) }
$$

For the following questions, please give your best estimate:

1) Over your lifetime, on how many DAYS have you been deliberately trying to limit the amount of food you eat in order to maintain or achieve a significantly low body weight (a weight that is less than minimally recommended)?

2) How many DAYS in the past two weeks?

3) Over your lifetime, on how many days have you gone for long periods of time (8 waking hours or more) without eating anything at all in order to influence your shape or weight?

4) How many days in the past two weeks?

5) Over your lifetime, on how many days have episodes of binge eating occurred? [Binge eating is defined as eating what other people would regard as an unusually large amount of food (given the circumstances) and having a sense of having lost control over your eating (at the time that you were eating).]

6) How many days in the past two weeks

7) Over your lifetime, how many TIMES have you made yourself sick (vomit) as a means of controlling your shape or weight?

8) How many TIMES in the past two weeks?

9) How many times in your life have you engaged in nonsuicidal self-injury? [Nonsuicidal self-injury is defined as purposely hurting yourself without wanting to die (for example, cutting or burning).]

10) How many times in the past two weeks?

11) How many separate times in your life have you had thoughts of killing yourself?

12) How many separate times in the past two weeks?

13) How many suicide attempts have you made in your lifetime? [A suicide attempt is defined as an actual attempt to kill yourself in which you had at least some intent to die.]

14) How many have you made in the past two weeks? 
APPENDIX F

OUTPUT FOR FITTED TWO-WEEK MODEL 


\section{Notes for Model (Default model)}

\section{Computation of degrees of freedom (Default model)}

Number of distinct sample moments: 253

Number of distinct parameters to be estimated: 53

Degrees of freedom (253 - 53): 200

\section{Result (Default model)}

Minimum was achieved

Chi-square $=275.616$

Degrees of freedom $=200$

Probability level $=.000$

\section{Maximum Likelihood Estimates}

Regression Weights: (Group number 1 - Default model)

\begin{tabular}{|c|c|c|c|c|c|c|}
\hline & & & Estimate & S.E. & C.R. & $\mathrm{P}$ \\
\hline Distress_Tolerance & $<--$ & Emotional_Reactivity & -.581 & .055 & -10.551 & $* * *$ \\
\hline Distress_Tolerance & $<--$ & Learned_Helplessness & -.117 & .024 & -4.776 & $* * *$ \\
\hline Behaviors & $<---$ & Distress_Tolerance & -.035 & .011 & -3.220 & .001 \\
\hline ER20 & $<---$ & Emotional_Reactivity & 1.000 & & & \\
\hline ER19 & $<---$ & Emotional_Reactivity & .853 & .061 & 13.965 & $* * *$ \\
\hline ER15 & $<---$ & Emotional_Reactivity & .763 & .063 & 12.173 & $* * *$ \\
\hline ER14 & $<--$ & Emotional_Reactivity & .739 & .065 & 11.376 & $* * *$ \\
\hline ER11 & $<---$ & Emotional_Reactivity & .827 & .063 & 13.034 & $* * *$ \\
\hline LHS_SU & $<---$ & Learned_Helplessness & 1.000 & & & \\
\hline LHS_C & $<--$ & Learned_Helplessness & .222 & .028 & 7.922 & $* * *$ \\
\hline LHS_IE & $<---$ & Learned_Helplessness & .726 & .065 & 11.132 & $* * *$ \\
\hline LHS_GS & $<---$ & Learned_Helplessness & .840 & .063 & 13.274 & $* * *$ \\
\hline LHS_AI & $<---$ & Learned_Helplessness & .282 & .030 & 9.411 & $* * *$ \\
\hline IDTS_App & $<---$ & Distress_Tolerance & .779 & .044 & 17.913 & $* * *$ \\
\hline LnSDB6 & $<--$ & Behaviors & 2.155 & .539 & 3.994 & $* * *$ \\
\hline LnSDB8 & $<--$ & Behaviors & 1.848 & .353 & 5.242 & $* * *$ \\
\hline LnSDB2 & $<---$ & Behaviors & 2.894 & .721 & 4.016 & $* * *$ \\
\hline LnSDB4 & $<---$ & Behaviors & 2.984 & .648 & 4.608 & $* * *$ \\
\hline LnSDB10 & $<--$ & Behaviors & 1.983 & .319 & 6.209 & $* * *$ \\
\hline LnSDB12 & $<--$ & Behaviors & 1.886 & .505 & 3.738 & $* * *$ \\
\hline LnSDB14 & $<--$ & Behaviors & 1.000 & & & \\
\hline ER5 & $<--$ & Emotional_Reactivity & .708 & .063 & 11.241 & $* * *$ \\
\hline IDTS_Abs & $<---$ & Distress_Tolerance & 1.000 & & & \\
\hline IDTS_Reg & $<--$ & Distress_Tolerance & .759 & .057 & 13.339 & $* * *$ \\
\hline IDTS_Tol & $<---$ & Distress_Tolerance & .892 & .047 & 18.966 & $* * *$ \\
\hline
\end{tabular}


Standardized Regression Weights: (Group number 1 - Default model)

\begin{tabular}{|lll|r|}
\hline & & & Estimate \\
\hline Distress_Tolerance & $<---$ & Emotional_Reactivity & -.659 \\
Distress_Tolerance & $<---$ & Learned_Helplessness & -.274 \\
Behaviors & $<---$ & Distress_Tolerance & -.266 \\
ER20 & $<---$ & Emotional_Reactivity & .857 \\
ER19 & $<---$ & Emotional_Reactivity & .750 \\
ER15 & $<---$ & Emotional_Reactivity & .681 \\
ER14 & $<---$ & Emotional_Reactivity & .642 \\
ER11 & $<---$ & Emotional_Reactivity & .711 \\
LHS_SU & $<---$ & Learned_Helplessness & .816 \\
LHS_C & $<---$ & Learned_Helplessness & .494 \\
LHS_IE & $<---$ & Learned_Helplessness & .672 \\
LHS_GS & $<---$ & Learned_Helplessness & .795 \\
LHS_AI & $<---$ & Learned_Helplessness & .579 \\
IDTS_App & $<---$ & Distress_Tolerance & .825 \\
LnSDB6 & $<---$ & Behaviors & .347 \\
LnSDB8 & $<---$ & Behaviors & .789 \\
LnSDB2 & $<---$ & Behaviors & .353 \\
LnSDB4 & $<---$ & Behaviors & .448 \\
LnSDB10 & $<---$ & Behaviors & .696 \\
LnSDB12 & $<---$ & Behaviors & .314 \\
LnSDB14 & $<---$ & Behaviors & .409 \\
ER5 & $<---$ & Emotional_Reactivity & .634 \\
IDTS_Abs & $<---$ & Distress_Tolerance & .894 \\
IDTS_Reg & $<---$ & Distress_Tolerance & .737 \\
IDTS_Tol & $<---$ & Distress_Tolerance & .853 \\
\hline & & &
\end{tabular}

Covariances: (Group number 1 - Default model)

\begin{tabular}{|c|c|c|c|c|c|c|}
\hline & Estimate & S.E. & C.R. & $\mathrm{P}$ \\
\hline Emotional_Reactivity & $\langle--\rangle$ & Learned_Helplessness & 1.232 & .191 & 6.462 & $* * *$ \\
\hline e18 & $<-->$ & e19 & .339 & .049 & 6.883 & $* * *$ \\
\hline $\mathrm{e} 12$ & $\langle-->$ & e14 & -.085 & .026 & -3.269 & .001 \\
\hline e22 & $\langle->$ & e24 & .016 & .006 & 2.802 & .005 \\
\hline e5 & $<-->$ & $\mathrm{e} 4$ & .141 & .048 & 2.960 & .003 \\
\hline e4 & $<-->$ & e3 & .250 & .056 & 4.470 & $* * *$ \\
\hline
\end{tabular}


Correlations: (Group number 1 - Default model)

\begin{tabular}{|ll|r|}
\hline & & Estimate \\
\hline Emotional_Reactivity & $<->$ Learned_Helplessness & .528 \\
e18 & $<->$ e19 & .502 \\
e12 & $<-->$ e14 & -.280 \\
e22 & $<-->$ e24 & .236 \\
e5 & $<->$ e4 & .203 \\
e4 & $<->$ e3 & .307 \\
\hline
\end{tabular}

Variances: (Group number 1 - Default model)

\begin{tabular}{|l|rrrr|}
\hline & Estimate & S.E. & C.R. & P \\
\hline Emotional_Reactivity & 1.126 & .132 & 8.510 & $* * *$ \\
Learned_Helplessness & 4.829 & .630 & 7.663 & $* * *$ \\
e16 & .263 & .039 & 6.762 & $* * *$ \\
e17 & .014 & .005 & 2.918 & .004 \\
e6 & .409 & .053 & 7.645 & $* * *$ \\
e5 & .639 & .065 & 9.788 & $* * *$ \\
e4 & .757 & .073 & 10.388 & $* * *$ \\
e3 & .879 & .082 & 10.694 & $* * *$ \\
e2 & .754 & .074 & 10.244 & $* * *$ \\
e11 & 2.419 & .318 & 7.601 & $* * *$ \\
e10 & .733 & .066 & 11.101 & $* * *$ \\
e9 & 3.084 & .306 & 10.069 & $* * *$ \\
e8 & 1.981 & .243 & 8.145 & $* * *$ \\
e7 & .764 & .071 & 10.736 & $* * *$ \\
e12 & .219 & .031 & 7.034 & $* * *$ \\
e13 & .249 & .026 & 9.690 & $* * *$ \\
e14 & .424 & .043 & 9.879 & $* * *$ \\
e15 & .262 & .029 & 9.104 & $* * *$ \\
e20 & .499 & .044 & 11.236 & $* * *$ \\
e21 & .031 & .006 & 5.073 & $* * *$ \\
e18 & .872 & .078 & 11.194 & $* * *$ \\
e19 & .523 & .048 & 10.783 & $* * *$ \\
e22 & .062 & .008 & 7.390 & $* * *$ \\
e23 & .481 & .042 & 11.343 & $* * *$ \\
e24 & .073 & .007 & 10.674 & $* * *$ \\
e1 & .837 & .078 & 10.763 & $* * *$ \\
\hline
\end{tabular}


Squared Multiple Correlations: (Group number 1 - Default model)

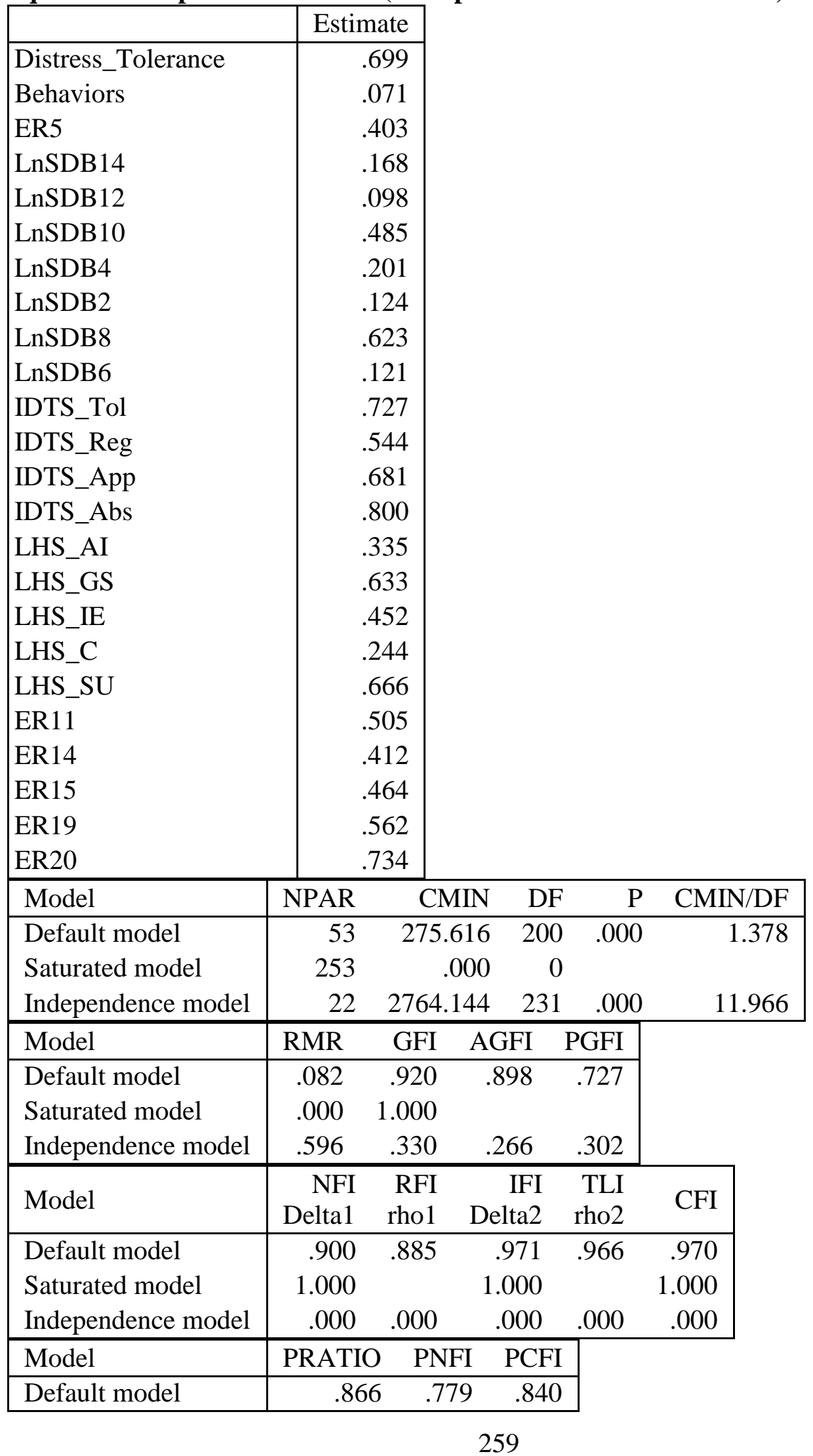




\begin{tabular}{|c|c|c|c|c|c|}
\hline Model & PRATIO & PNFI & PCFI & & \\
\hline Saturated model & .000 & .000 & .000 & & \\
\hline Independence model & 1.000 & .000 & .000 & & \\
\hline Model & $\mathrm{NCP}$ & \multicolumn{2}{|c|}{ LO 90} & HI 90 & \\
\hline Default model & 75.616 & \multicolumn{2}{|c|}{36.005} & 123.280 & \\
\hline Saturated model & .000 & \multicolumn{2}{|c|}{.000} & .000 & \\
\hline Independence model & 2533.144 & \multicolumn{2}{|c|}{2367.460} & 706.188 & \\
\hline Model & FMIN & F0 & LO 90 & HI 90 & \\
\hline Default model & .999 & .274 & .130 & .447 & \\
\hline Saturated model & .000 & .000 & .000 & .000 & \\
\hline Independence model & 10.015 & 9.178 & 8.578 & 9.805 & \\
\hline Model & RMSEA & LO 90 & HI 90 & PCLOSE & SE \\
\hline Default model & .037 & .026 & .047 & & 83 \\
\hline Independence model & .199 & .193 & .206 & & 00 \\
\hline Model & $\mathrm{AIC}$ & \multicolumn{2}{|c|}{$\mathrm{BCC}$} & $\mathrm{BIC}$ & CAIC \\
\hline Default model & 381.616 & \multicolumn{2}{|c|}{391.252} & 573.689 & 626.689 \\
\hline Saturated model & 506.000 & \multicolumn{2}{|c|}{552.000} & 1422.876 & 1675.876 \\
\hline Independence model & 2808.144 & 2812. & 44 & 387.872 & 2909.872 \\
\hline Model & ECVI & LO 90 & HI 90 & MECVI & \\
\hline Default model & 1.383 & 1.239 & 1.555 & 1.418 & \\
\hline Saturated model & 1.833 & 1.833 & 1.833 & 2.000 & \\
\hline Independence model & 10.174 & 9.574 & 10.801 & 10.189 & \\
\hline Model & $\begin{array}{r}\text { HOELTER } \\
.05 \\
\end{array}$ & $\begin{array}{ll}\mathrm{R} & \mathrm{HO} \\
5 & \\
\end{array}$ & $\begin{array}{r}\text { ELTER } \\
.01\end{array}$ & & \\
\hline Default model & 235 & 5 & 250 & & \\
\hline Independence model & & 7 & 29 & & \\
\hline
\end{tabular}


APPENDIX G

OUTPUT FOR FITTED LIFETIME MODEL 
Number of distinct sample moments:

378

Number of distinct parameters to be estimated: $\quad 66$

Degrees of freedom (378 - 66): 312

\begin{tabular}{|c|c|c|c|c|c|c|}
\hline & & & Estimate & S.E. & C.R. & $\mathrm{P}$ \\
\hline Distress_Tolerance & $<---$ & Emotional_Reactivity & -.584 & .055 & -10.624 & $* * *$ \\
\hline Distress_Tolerance & $<---$ & Learned_Helplessness & -.118 & .025 & -4.741 & $* * *$ \\
\hline Behaviors & $<---$ & Distress_Tolerance & -.072 & .025 & -2.876 & .004 \\
\hline Behaviors & $<---$ & Depression & .142 & .034 & 4.140 & $* * *$ \\
\hline ER20 & $<---$ & Emotional_Reactivity & 1.000 & & & \\
\hline ER19 & $<---$ & Emotional_Reactivity & .854 & .060 & 14.115 & $* * *$ \\
\hline ER15 & $<--$ & Emotional_Reactivity & .766 & .062 & 12.343 & $* * *$ \\
\hline ER14 & $<---$ & Emotional_Reactivity & .737 & .065 & 11.404 & $* * *$ \\
\hline ER11 & $<--$ & Emotional_Reactivity & .821 & .063 & 13.012 & $* * *$ \\
\hline LHS_SU & $<--$ & Learned_Helplessness & 1.000 & & & \\
\hline LHS_C & $<---$ & Learned_Helplessness & .227 & .029 & 7.962 & $* * *$ \\
\hline LHS_IE & $<---$ & Learned_Helplessness & .741 & .067 & 11.129 & $* * *$ \\
\hline LHS_GS & $<---$ & Learned_Helplessness & .878 & .065 & 13.616 & $* * *$ \\
\hline LHS_AI & $<---$ & Learned_Helplessness & .282 & .031 & 9.180 & $* * *$ \\
\hline IDTS_App & $<---$ & Distress_Tolerance & .776 & .043 & 18.068 & $* * *$ \\
\hline IDTS_Reg & $<---$ & Distress_Tolerance & .755 & .057 & 13.348 & $* * *$ \\
\hline LnSDB9 & $<---$ & Behaviors & 4.022 & .611 & 6.585 & $* * *$ \\
\hline LnSDB11 & $<---$ & Behaviors & 6.141 & .906 & 6.776 & $* * *$ \\
\hline LnSDB13 & $<---$ & Behaviors & 1.000 & & & \\
\hline ER5 & $<---$ & Emotional_Reactivity & .704 & .063 & 11.231 & $* * *$ \\
\hline DEP7 & $<---$ & Depression & 1.000 & & & \\
\hline DEP6 & $<---$ & Depression & 1.049 & .068 & 15.353 & $* * *$ \\
\hline DEP5 & $<---$ & Depression & .993 & .077 & 12.859 & $* * *$ \\
\hline DEP4 & $<---$ & Depression & .846 & .072 & 11.668 & $* * *$ \\
\hline DEP3 & $<---$ & Depression & 1.051 & .079 & 13.392 & $* * *$ \\
\hline DEP2 & $<---$ & Depression & .851 & .078 & 10.916 & $* * *$ \\
\hline DEP1 & $<---$ & Depression & .822 & .068 & 12.099 & $* * *$ \\
\hline LnSDB7 & $<---$ & Behaviors & 1.611 & .499 & 3.227 & .001 \\
\hline LnSDB3 & $<---$ & Behaviors & 2.137 & .704 & 3.036 & .002 \\
\hline IDTS_Abs & $<---$ & Distress_Tolerance & 1.000 & & & \\
\hline IDTS_Tol & $<--$ & Distress_Tolerance & .885 & .046 & 19.051 & $* * *$ \\
\hline & & & Estimate & & & \\
\hline Distress_Tolerance & $<---$ & Emotional_Reactivity & -.659 & & & \\
\hline Distress_Tolerance & $<---$ & Learned_Helplessness & -.271 & & & \\
\hline Behaviors & $<---$ & Distress_Tolerance & -.253 & & & \\
\hline Behaviors & $<---$ & Depression & .412 & & & \\
\hline ER20 & $<---$ & Emotional_Reactivity & .858 & & & \\
\hline
\end{tabular}




\begin{tabular}{|c|c|c|c|c|c|}
\hline & & Estimate & & & \\
\hline ER19 & <--- Emotional_Reactivity & .751 & & & \\
\hline ER15 & <--- Emotional_Reactivity & 685 & & & \\
\hline ER14 & <--- Emotional_Reactivity & .640 & & & \\
\hline ER11 & <--- Emotional_Reactivity & .707 & & & \\
\hline LHS_SU & <--- Learned_Helplessness & .800 & & & \\
\hline LHS_C & <--- Learned_Helplessness & 497 & & & \\
\hline LHS_IE & <--- Learned_Helplessness & 673 & & & \\
\hline LHS_GS & <--- Learned_Helplessness & .815 & & & \\
\hline LHS_AI & <--- Learned_Helplessness & .566 & & & \\
\hline IDTS_App & <--- Distress_Tolerance & .825 & & & \\
\hline IDTS_Reg & <--- Distress_Tolerance & .737 & & & \\
\hline LnSDB9 & $<---\quad$ Behaviors & .656 & & & \\
\hline LnSDB11 & $<---$ Behaviors & .774 & & & \\
\hline LnSDB13 & $<---\quad$ Behaviors & .498 & & & \\
\hline ER5 & <--- Emotional_Reactivity & .632 & & & \\
\hline DEP7 & <--- Depression & .732 & & & \\
\hline DEP6 & <--- Depression & .769 & & & \\
\hline DEP5 & <--- Depression & .794 & & & \\
\hline DEP4 & $<---$ Depression & .722 & & & \\
\hline DEP3 & <--- Depression & .826 & & & \\
\hline DEP2 & $<---$ Depression & .677 & & & \\
\hline DEP1 & $<---$ Depression & .748 & & & \\
\hline LnSDB7 & $<--$ Behaviors & .238 & & & \\
\hline LnSDB3 & $<---\quad$ Behaviors & .222 & & & \\
\hline IDTS_Abs & <--- Distress_Tolerance & .899 & & & \\
\hline \multirow[t]{2}{*}{ IDTS_Tol } & <--- Distress_Tolerance & .850 & & & \\
\hline & & Estimate & S.E. & C.R. & $\mathrm{P}$ \\
\hline Emotional_Reactivity & y 〈--> Learned_Helplessne & 1.209 & .188 & 6.439 & **** \\
\hline Learned_Helplessnes & ss <--> Depression & 1.076 & .152 & 7.083 & $* * *$ \\
\hline Emotional_Reactivity & y <--> Depression & .505 & .071 & 7.108 & $* * *$ \\
\hline e12 & $<-->$ e14 & -.088 & .026 & -3.403 & $* * *$ \\
\hline e29 & $<-->\quad$ e28 & .161 & .037 & 4.380 & $* * *$ \\
\hline e19 & $\langle-->\quad$ e18 & 1.127 & .278 & 4.053 & $* * *$ \\
\hline e4 & $<-->$ e3 & .248 & .056 & 4.453 & $* * *$ \\
\hline \multirow[t]{2}{*}{ e5 } & $\langle-->$ e4 & .135 & .047 & 2.874 & .004 \\
\hline & & Estimate & & & \\
\hline Emotional_Reactivity & y 〈--> Learned_Helplessne & .528 & & & \\
\hline Learned_Helplessnes & ss <--> Depression & .648 & & & \\
\hline Emotional_Reactivity & $y<-->$ Depression & .616 & & & \\
\hline e12 & $<-->$ e14 & -.294 & & & \\
\hline
\end{tabular}




\begin{tabular}{|c|c|c|c|c|c|}
\hline & & & & & Estimate \\
\hline $\mathrm{e} 29$ & $\langle--\rangle$ & $\mathrm{e} 28$ & & & .333 \\
\hline e19 & $\langle--\rangle$ & e18 & & & .257 \\
\hline e4 & $\langle-->$ & e3 & & & .305 \\
\hline \multirow[t]{2}{*}{ e5 } & $\langle--\rangle$ & e4 & & & .196 \\
\hline & & Estimate & S.E. & C.R. & $\mathrm{P}$ \\
\hline Emotional_Reactivity & & 1.129 & .132 & 8.554 & $* * *$ \\
\hline Learned_Helplessness & & 4.639 & .618 & 7.510 & $* * *$ \\
\hline Depression & & .594 & .087 & 6.818 & $* * *$ \\
\hline e16 & & 268 & .039 & 6.891 & $* * *$ \\
\hline e17 & & .046 & .012 & 3.693 & $* * *$ \\
\hline e6 & & .406 & .052 & 7.748 & $* * *$ \\
\hline e5 & & .635 & .065 & 9.836 & $* * *$ \\
\hline e4 & & .750 & .072 & 10.407 & $* * *$ \\
\hline e3 & & .882 & .082 & 10.737 & $* * *$ \\
\hline e2 & & .763 & .074 & 10.332 & $* * *$ \\
\hline e11 & & 2.610 & .315 & 8.271 & $* * *$ \\
\hline e10 & & .731 & .066 & 11.131 & $* * *$ \\
\hline e9 & & 3.079 & .303 & 10.165 & $* * *$ \\
\hline e8 & & 1.814 & .230 & 7.903 & $* * *$ \\
\hline e7 & & .780 & .072 & 10.857 & $* * *$ \\
\hline e12 & & .211 & .031 & 6.884 & $* * *$ \\
\hline e13 & & 249 & .026 & 9.725 & $* * *$ \\
\hline e14 & & .424 & .043 & 9.878 & $* * *$ \\
\hline e15 & & .266 & .029 & 9.206 & $* * *$ \\
\hline e20 & & 1.517 & .177 & 8.590 & $* * *$ \\
\hline e21 & & 1.793 & .305 & 5.872 & $* * *$ \\
\hline e22 & & .215 & .021 & 10.469 & $* * *$ \\
\hline e1 & & .842 & .078 & 10.812 & $* * *$ \\
\hline e29 & & .516 & .050 & 10.275 & $* * *$ \\
\hline e28 & & .452 & .045 & 9.949 & $* * *$ \\
\hline e27 & & .344 & .035 & 9.734 & $* * *$ \\
\hline e26 & & .390 & .037 & 10.466 & $* * *$ \\
\hline e25 & & .305 & .033 & 9.196 & $* * *$ \\
\hline e24 & & .509 & .047 & 10.755 & $* * *$ \\
\hline e23 & & .316 & .031 & 10.251 & $* * *$ \\
\hline e19 & & 3.072 & .267 & 11.522 & $* * *$ \\
\hline \multirow[t]{2}{*}{ e18 } & & 6.248 & .541 & 11.552 & $* * *$ \\
\hline & & stimate & & & \\
\hline Distress_Tolerance & & .698 & & & \\
\hline Behaviors & & .355 & & & \\
\hline
\end{tabular}




\begin{tabular}{|c|c|c|c|c|c|c|}
\hline & \multicolumn{2}{|c|}{ Estimate } & & & & \\
\hline LnSDB3 & & .049 & & & & \\
\hline LnSDB7 & & .056 & & & & \\
\hline DEP1 & & .560 & & & & \\
\hline DEP2 & & .458 & & & & \\
\hline DEP3 & & .683 & & & & \\
\hline DEP4 & & .522 & & & & \\
\hline DEP5 & & 630 & & & & \\
\hline DEP6 & & .592 & & & & \\
\hline DEP7 & & .535 & & & & \\
\hline ER5 & & .399 & & & & \\
\hline LnSDB13 & & 248 & & & & \\
\hline LnSDB11 & & 598 & & & & \\
\hline LnSDB9 & & 430 & & & & \\
\hline IDTS_Tol & & .723 & & & & \\
\hline IDTS_Reg & & 543 & & & & \\
\hline IDTS_App & & 681 & & & & \\
\hline IDTS_Abs & & 808 & & & & \\
\hline LHS_AI & & 320 & & & & \\
\hline LHS_GS & & 664 & & & & \\
\hline LHS_IE & & 453 & & & & \\
\hline LHS_C & & 247 & & & & \\
\hline LHS_SU & & 640 & & & & \\
\hline ER11 & & 499 & & & & \\
\hline ER14 & & 410 & & & & \\
\hline ER15 & & 469 & & & & \\
\hline ER19 & & .565 & & & & \\
\hline ER20 & & 736 & & & & \\
\hline Model & NPAR & $\mathrm{CM}$ & IN $\quad$ DF & $\mathrm{P}$ & CMII & IN/DF \\
\hline Default model & 66 & 455.6 & $86 \quad 312$ & .000 & & 1.461 \\
\hline Saturated model & 378 & & 00 & & & \\
\hline Independence model & 27 & 4016.1 & $75 \quad 351$ & $1 \quad .000$ & & 11.442 \\
\hline Model & RMR & GFI & AGFI & PGFI & & \\
\hline Default model & .109 & .894 & .872 & .738 & & \\
\hline Saturated model & .000 & 1.000 & & & & \\
\hline Independence model & .626 & .237 & .178 & .220 & & \\
\hline Model & $\begin{array}{r}\text { NFI } \\
\text { Delta1 }\end{array}$ & $\begin{array}{c}\text { RFI } \\
\text { rho1 }\end{array}$ & $\begin{array}{r}\text { IFI } \\
\text { Delta2 }\end{array}$ & $\begin{array}{r}\text { TLI } \\
\text { rho2 } \\
\end{array}$ & CFI & \\
\hline Default model & .887 & .872 & .961 & .956 & .961 & \\
\hline Saturated model & 1.000 & & 1.000 & & 1.000 & \\
\hline Independence model & .000 & .000 & .000 & .000 & .000 & \\
\hline
\end{tabular}




\begin{tabular}{|c|c|c|c|c|c|}
\hline Model & PRATIO & PNFI & PCFI & & \\
\hline Default model & .889 & .788 & .854 & & \\
\hline Saturated model & .000 & .000 & .000 & & \\
\hline Independence model & 1.000 & .000 & .000 & & \\
\hline Model & $\mathrm{NCP}$ & \multicolumn{2}{|c|}{ LO 90} & HI 90 & \\
\hline Default model & 143.686 & \multicolumn{2}{|c|}{90.701} & 204.669 & \\
\hline Saturated model & .000 & \multicolumn{2}{|c|}{.000} & \multirow{2}{*}{$\begin{array}{r}.000 \\
872.783 \\
\end{array}$} & \\
\hline Independence model & 3665.175 & \multicolumn{2}{|c|}{3464.880} & & \\
\hline Model & FMIN & $\mathrm{F} 0$ & LO 90 & HI 90 & \\
\hline Default model & 1.651 & .521 & .329 & .742 & \\
\hline Saturated model & .000 & .000 & .000 & .000 & \\
\hline Independence model & 14.551 & 13.280 & 12.554 & 14.032 & \\
\hline Model & RMSEA & LO 90 & HI 90 & PCLOSE & \\
\hline Default model & .041 & .032 & .049 & .972 & \\
\hline Independence model & .195 & .189 & .200 & \multicolumn{2}{|c|}{.000} \\
\hline Model & AIC & \multicolumn{2}{|c|}{$\mathrm{BCC}$} & $\mathrm{BIC}$ & CAIC \\
\hline Default model & 587.686 & \multicolumn{2}{|c|}{602.589} & 826.871 & 892.871 \\
\hline Saturated model & 756.000 & \multicolumn{2}{|c|}{841.355} & 2125.879 & 2503.879 \\
\hline Independence model & 4070.175 & \multicolumn{2}{|c|}{4076.272} & 168.023 & +195.023 \\
\hline Model & ECVI & LO 90 & HI 90 & $0 \quad$ MECVI & \\
\hline Default model & 2.129 & 1.937 & 2.350 & 2.183 & \\
\hline Saturated model & 2.739 & 2.739 & 2.739 & 3.048 & \\
\hline Independence model & 14.747 & 14.021 & 15.499 & 14.769 & \\
\hline Model & $\begin{array}{r}\text { HOELTE } \\
.0\end{array}$ & $\begin{array}{ll}\mathrm{R} & \mathrm{HOH} \\
5 & \end{array}$ & $\begin{array}{r}\text { ETER } \\
.01\end{array}$ & & \\
\hline Default model & 21 & & 226 & & \\
\hline Independence model & & 28 & 29 & & \\
\hline
\end{tabular}




\section{REFERENCES}




\section{References}

Abramson, L., Seligman, M., \& Teasdale, J. (1978). Learned helplessness in humans: Critique and reformulation. Journal of Abnormal Psychology, 87(1), 49-74.

Allan, N., Macatee, R., Norr, A., \& Schmidt, N. (2014). Direct and interactive effects of distress tolerance and anxiety sensitivity on generalized anxiety and depression. Cognitive Therapy \& Research, 38(5), 530-540. doi:10.1007/s10608-014-9623-y

Allen, J., Harmon-Jones, E., \& Cavender, J. (2001). Manipulation of frontal EEG asymmetry through biofeedback alters self-reported emotional responses and facial EMG. Psychophysiology, 38(4), 685-693.

American Psychiatric Association. (2000). Diagnostic and statistical manual of mental disorders: DSM-IV-TR (4th ed., text rev.). Washington, DC: Author.

American Psychiatric Association. (2010). Practice guideline for the treatment of patients with major depressive disorder (3rd ed.). Washington, DC: Author.

American Psychiatric Association. (2013). Diagnostic and statistical manual of mental disorders: DSM-5 (5th ed.). Washington, DC: Author.

Amstadter, A., Daughters, S., Macpherson, L., Reynolds, E., Danielson, C., Wang, F., . . . Lejuez, C. (2012). Genetic associations with performance on a behavioral measure of distress intolerance. Journal of Psychiatric Research, 46(1), 87-94. doi:10.1016/j.jpsychires.2011.09.017

Andover, M., \& Gibb, B. (2010). Non-suicidal self-injury, attempted suicide, and suicidal intent among psychiatric inpatients. Psychiatry Research, 178(1), 101-105. doi:10.1016/j.psychres.2010.03.019

Andover, M., Primack, J., Gibb, B., \& Pepper, C. (2010). An examination of non-suicidal self-injury in men: Do men differ from women in basic NSSI characteristics? Archives of Suicide Research, 14(1), 79-88. doi:10.1080/13811110903479086

Anestis, M., Bagge, C., Tull, M., \& Joiner, T. (2011). Clarifying the role of emotion dysregulation in the interpersonal-psychological theory of suicidal behavior in an undergraduate sample. Journal of Psychiatric Research, 45(5), 603-611. doi:10.1016/j.jpsychires.2010.10.013 
Anestis, M., Bender, T., Selby, E., Ribeiro, J., \& Joiner, T. (2011). Sex and emotion in the acquired capability for suicide. Archives of Suicide Research, 15(2), 172-182. doi:10.1080/13811118.2011.566058

Anestis, M., \& Joiner, T. (2012). Behaviorally-indexed distress tolerance and suicidality. Journal of Psychiatric Research, 46(6), 703-707.

doi:10.1016/j.jpsychires.2012.02.015

Anestis, M., Kleiman, E., Lavender, J., Tull, M., \& Gratz, K. (2014). The pursuit of death versus escape from negative affect: An examination of the nature of the relationship between emotion dysregulation and both suicidal behavior and nonsuicidal self-injury. Comprehensive Psychiatry, 55(8), 1820-1830.

doi:10.1016/j.comppsych.2014.07.007

Anestis, M., Knorr, A., Tull, M., Lavender, J., \& Gratz, K. (2013). The importance of high distress tolerance in the relationship between nonsuicidal self-injury and suicide potential. Suicide \& Life-Threatening Behavior, 43(6), 663-675. doi:10.1111/sltb.12048

Anestis, M., Lavender, J., Marshall-Berenz, E., Gratz, K., Tull, M., \& Joiner, T. (2012). Evaluating distress tolerance measures: Interrelations and associations with impulsive behaviors. Cognitive Therapy \& Research, 36(6), 593-602. doi:10.1007/s10608-011-9377-8

Anestis, M., Moberg, F., \& Arnau, R. (2014). Hope and the interpersonal-psychological theory of suicidal behavior: Replication and extension of prior findings. Suicide \& Life-Threatening Behavior, 44(2), 175-187. doi:10.1111/sltb.12060

Anestis, M., Pennings, S., Lavender, J., Tull, M., \& Gratz, K. (2013). Low distress tolerance as an indirect risk factor for suicidal behavior: Considering the explanatory role of non-suicidal self-injury. Comprehensive Psychiatry, 54(7), 996-1002. doi:10.1016/j.comppsych.2013.04.005

Anestis, M., Selby, E., Fink, E., \& Joiner, T. (2007). The multifaceted role of distress tolerance in dysregulated eating behaviors. International Journal of Eating Disorders, 40(8), 718-726. doi:10.1002/eat.20471

Antony, M., Bieling, P., Cox, B., Enns, M., \& Swinson, R. (1998). Psychometric properties of the 42-item and 21-item versions of the Depression Anxiety Stress Scales in clinical groups and a community sample. Psychological Assessment, 10(2), 176-181. doi:10.1037/1040-3590.10.2.176

Arbuckle, J. (2016). Amos (Version 24.0). [Computer Program]. IBM SPSS. Chicago.

Baník, G., \& Gajdošová, B. (2014). Positive changes following cancer: Posttraumatic growth in the context of other factors in patients with cancer. Supportive Care in Cancer, 22(8), 2023-2029. doi:10.1007/s00520-014-2217-0 
Bardeen, J., Fergus, T., \& Orcutt, H. (2013). Testing a hierarchical model of distress tolerance. Journal of Psychopathology \& Behavioral Assessment, 35(4), 495-505. doi:10.1007/s10862-013-9359-0

Bardeen, J., Tull, M., Dixon-Gordon, K., Stevens, E., \& Gratz, K. (2015). Attentional control as a moderator of the relationship between difficulties accessing effective emotion regulation strategies and distress tolerance. Journal of Psychopathology \& Behavioral Assessment, 37(1), 79-84. doi:10.1007/s10862-014-9433-2

Barth, J., Munder, T., Gerger, H., Nüesch, E., Trelle, S., Znoj, H., . . Cuijpers, P. (2013). Comparative efficacy of seven psychotherapeutic interventions for patients with depression: A network meta-analysis. PLOS Medicine, 10(5), e1001454. doi:10.1371/journal.pmed.1001454

Bebane, S., Flowe, H., \& Maltby, J. (2015). Re-refining the measurement of distress intolerance. Personality \& Individual Differences, 85, 159-164. doi:10.1016/j.paid.2015.05.005

Beck, K., Daughters, S., \& Ali, B. (2013). Hurried driving: Relationship to distress tolerance, driver anger, aggressive and risky driving in college students. Accident Analysis \& Prevention, 51, 51-55. doi:10.1016/j.aap.2012.10.012

Bender, T., Anestis, M., Anestis, J., Gordon, K., \& Joiner, T. (2012). Affective and behavioral paths toward the acquired capacity for suicide. Journal of Social \& Clinical Psychology, 31(1), 81-100. doi:10.1521/jscp.2012.31.1.81

Berg, K., Peterson, C., Frazier, P., \& Crow, S. (2012). Psychometric evaluation of the Eating Disorder Examination and Eating Disorder Examination-Questionnaire: A systematic review of the literature. International Journal of Eating Disorders, 45(3), 428-438. doi:10.1002/eat.20931

Bernstein, A., Zvolensky, M., Vujanovic, A., \& Moos, R. (2009). Integrating anxiety sensitivity, distress tolerance, and discomfort intolerance: A hierarchical model of affect sensitivity and tolerance. Behavior Therapy, 40(3), 291-301. doi:10.1016/j.beth.2008.08.001

Booth, R., Keogh, K., Doyle, J., \& Owens, T. (2014). Living through distress: A skills training group for reducing deliberate self-harm. Behavioural \& Cognitive Psychotherapy, 42(2), 156-165. doi:10.1017/S1352465812001002

Bornovalova, M., Gratz, K., Daughters, S., Hunt, E., \& Lejuez, C. (2012). Initial RCT of a distress tolerance treatment for individuals with substance use disorders. Drug \& Alcohol Dependence, 122(1-2), 70-76. doi:10.1016/j.drugalcdep.2011.09.012

Bornovalova, M., Gratz, K., Daughters, S., Nick, B., Delany-Brumsey, A., Lynch, T., . . . Lejuez, C. (2008). A multimodal assessment of the relationship between emotion dysregulation and borderline personality disorder among inner-city substance 
users in residential treatment. Journal of Psychiatric Research, 42(9), 717-726. doi:10.1016/j.jpsychires.2007.07.014

Bornovalova, M., Matusiewicz, A., \& Rojas, E. (2011). Distress tolerance moderates the relationship between negative affect intensity with borderline personality disorder levels. Comprehensive Psychiatry, 52(6), 744-753.

doi:10.1016/j.comppsych.2010.11.005

Brandt, C., Zvolensky, M., \& Bonn-Miller, M. (2013). Distress tolerance, emotion dysregulation, and anxiety and depressive symptoms among HIV+ individuals. Cognitive Therapy \& Research, 37(3), 446-455. doi:10.1007/s10608-012-9497-9

Braun, S., \& Turner, R. (2014). Attitudes and company practices as predictors of managers' intentions to hire, develop, and promote women in science, engineering, and technology professions. Consulting Psychology Journal: Practice and Research, 66(2), 93-117. doi:10.1037/a0037079

Britton, W., Shahar, B., Szepsenwol, O., \& Jacobs, W. (2012). Mindfulness-based cognitive therapy improves emotional reactivity to social stress: Results from a randomized controlled trial. Behavior Therapy, 43(2), 365-380. doi:10.1016/j.beth.2011.08.006

Brown, R., Lejuez, C., Strong, D., Kahler, C., Zvolensky, M., Carpenter, L., . . Price, L. (2009). A prospective examination of distress tolerance and early smoking lapse in adult self-quitters. Nicotine \& Tobacco Research, 11(5), 493-502. doi:10.1093/ntr/ntp041

Buchanan, E., \& Hvizdak, E. (2009). Online survey tools: Ethical and methodological concerns of human research ethics committees. Journal of Empirical Research on Human Research Ethics, 4(2), 37-48. doi:10.1525/jer.2009.4.2.37

Buckner, J. D., Keough, M. E., \& Schmidt, N. B. (2007). Problematic alcohol and cannabis use among young adults: The roles of depression and discomfort and distress tolerance. Addictive Behaviors, 32(9), 1957-1963. doi:10.1016/j.addbeh.2006.12.019

Buhi, E., Goodson, P., \& Neilands, T. (2007). Structural equation modeling: A primer for health behavior researchers. American Journal of Health Behavior, 31(1), 74-85. doi:10.5993/AJHB.31.1.8

Bylsma, L., Morris, B., \& Rottenburg, J. (2008). A meta-analysis of emotional reactivity in major depressive disorder. Clinical Psychology Review, 28, 676-391. doi:10.1016/j.cpr.2007.10.001

Byrne, S. (2010). Structural equation modeling with AMOS: Basic concepts, applications, and programming (2nd ed.). New York, NY: Routledge. 
Capron, D., Norr, A., Macatee, R., \& Schmidt, N. (2013). Distress tolerance and anxiety sensitivity cognitive concerns: Testing the incremental contributions of affect dysregulation constructs on suicidal ideation and suicide attempt. Behavior Therapy, 44(3), 349-358. doi:10.1016/j.beth.2012.12.002

Cawthon, S. (2011). Making decisions about assessment practices for students who are deaf or hard of hearing. Remedial and Special Education, 32(1), 4-21. doi: $10.1177 / 0741932509355950$

Center for Disease Control. (2015). Suicide: Facts at a glance. Retrieved from http://www.cdc.gov/violenceprevention/pdf/suicide-datasheet-a.pdf

Cha, C., Najmi, S., Park, J., Finn, C., \& Nock, M. (2010). Attentional bias toward suicide-related stimuli predicts suicidal behavior. Journal of Abnormal Psychology, 119(3), 616-622. doi:10.1037/a0019710

Chapman, A., \& Dixon-Gordon, K. (2007). Emotional antecedents and consequences of deliberate self-harm and suicide attempts. Suicide \& Life-Threatening Behavior, 37(5), 543-552. doi:10.1521/suli.2007.37.5.543

Chiappelli, J., Pocivavsek, A., Nugent, K., Notarangelo, F., Kochunov, P., Rowland, L., . .. Hong, L. (2014). Stress-induced increase in kynurenic acid as a potential biomarker for patients with schizophrenia and distress intolerance. JAMA Psychiatry, 71(7), 761-768. doi:10.1001/jamapsychiatry.2014.243

Claes, L., Smits, D., \& Bijttebier, P. (2014). The Dutch version of the Emotion Reactivity Scale: Validation and relation with various behaviors in a sample of high school students. European Journal of Psychological Assessment, 30(1), 73-79. doi:10.1027/1015-5759/a000171

Corstorphine, E., Mountford, V., Tomlinson, S., Waller, G., \& Meyer, C. (2007). Distress tolerance in the eating disorders. Eating Behaviors, 8(1), 91-97. doi:10.1016/j.eatbeh.2006.02.003

Cougle, J., Timpano, K., Fitch, K., \& Hawkins, K. (2011). Distress tolerance and obsessions: An integrative analysis. Depression \& Anxiety, 28(10), 906-914. doi:10.1002/da.20846

Cougle, J., Timpano, K., Sarawgi, S., Smith, C., \& Fitch, K. (2013). A multi-modal investigation of the roles of distress tolerance and emotional reactivity in obsessive-compulsive symptoms. Anxiety, Stress \& Coping, 26(5), 478-492. doi:10.1080/10615806.2012.697156

Creswell, J., Way, B., Eisenberger, N., \& Lieberman, M. (2007). Neural correlates of dispositional mindfulness during affect labeling. Psychosomatic medicine, 69(6), 560-565. doi:10.1097/PSY.0b013e3180f6171f 
Cummings, J., Bornovalova, M., Ojanen, T., Hunt, E., MacPherson, L., \& Lejuez, C. (2013). Time doesn't change everything: The longitudinal course of distress tolerance and its relationship with externalizing and internalizing symptoms during early adolescence. Journal of Abnormal Child Psychology, 41(5), 735-748. doi:10.1007/s10802-012-9704-x

Daughters, S., Gorka, S., Rutherford, H., \& Mayes, L. (2014). Maternal and adolescent distress tolerance: The moderating role of gender. Emotion, 14(2), 416-424. doi: $10.1037 / \mathrm{a} 0034991$

Daughters, S., Lejuez, C., Bornovalova, M., Kahler, C., Strong, D., \& Brown, R. (2005). Distress tolerance as a predictor of early treatment dropout in a residential substance abuse treatment facility. Journal of Abnormal Psychology, 114(4), 729734. doi:10.1037/0021-843X.114.4.729

Daughters, S., Reynolds, E., MacPherson, L., Kahler, C., Danielson, C., Zvolensky, M., $\&$ Lejuez, C. (2009). Distress tolerance and early adolescent externalizing and internalizing symptoms: The moderating role of gender and ethnicity. Behaviour Research \& Therapy, 47(3), 198-205. doi:10.1016/j.brat.2008.12.001

Daughters, S., Sargeant, M., Bornovalova, M., Gratz, K., \& Lejuez, C. (2008). The relationship between distress tolerance and antisocial personality disorder among male inner-city treatment seeking substance users. Journal of Personality Disorders, 22(5), 509-524. doi:10.1521/pedi.2008.22.5.509

Davidson, C., Wingate, L., Rasmussen, K., \& Slish, M. (2009). Hope as a predictor of interpersonal suicide risk. Suicide and Life-Threatening Behavior, 39(5), 499-507. doi:10.1521/suli.2009.39.5.499

Davidson, C., Wingate, L., Slish, M., \& Rasmus, K. (2010). The great black hope: Hope and its relation to suicide risk among African Americans. Suicide and LifeThreatening Behavior, 40(2), 170-180. doi:10.1521/suli.2010.40.2.170

Eisenberger, R., Park, D., \& Frank, M. (1976). Learned helplessness and social reinforcement. Journal of Personality and Social Psychology, 33(2), 227-232. doi:10.1037/0022-3514.33.2.227

Ellis, A., Fischer, K., \& Beevers, C. (2010). Is dysphoria about being red and blue? Potentiation of anger and reduced distress tolerance among dysphoric individuals. Cognition \& Emotion, 24(4), 596-608. doi:10.1080/13803390902851176

Ellis, A., Vanderlind, M., \& Beevers, C. (2013). Enhanced anger reactivity and reduced distress tolerance in major depressive disorder. Cognitive Therapy \& Research, 37(3), 498-509. doi:10.1007/s10608-012-9494-z

Evans, E., Hawton, K., \& Rodham, K. (2005). In what ways are adolescents who engage in self-harm or experience thoughts of self-harm different in terms of help- 
seeking, communication and coping strategies? Journal of Adolescence, 28(4), 573-587. doi:10.1016/j.adolescence.2004.11.001

Fairburn, C., \& Beglin, S. (2008). Eating Disorder Examination Questionnaire 6.0. In C. Fairburn (Ed.), Cognitive behavior therapy and eating disorders (pp. 311-313). New York, NY: Guilford Press.

Feldman, G., Dunn, E., Stemke, C., Bell, K., \& Greeson, J. (2014). Mindfulness and rumination as predictors of persistence with a distress tolerance task. Personality \& Individual Differences, 56, 154-158. doi:10.1016/j.paid.2013.08.040

Feldman, H. (1986). Self-esteem, types of attributional style and sensation and distress pain ratings in males. Journal of Advanced Nursing, 11(1), 75-86. doi:10.1111/1365-2648.ep13112622

Feliu-Soler, A., Pascual, J., Borras, X., Portella, M., Martin-Blanco, A., Armario, A., .. . Soler, J. (2014). Effects of dialectical behaviour therapy-mindfulness training on emotional reactivity in borderline personality disorder: Preliminary results. Clinical Psycholology \& Psychotherapy, 21(4), 363-370. doi:10.1002/cpp.1837

Fergus, T., Bardeen, J., \& Orcutt, H. (2015). Examining the specific facets of distress tolerance that are relevant to health anxiety. Journal of Cognitive Psychotherapy, 29(1), 32-44. doi:10.1891/0889-8391.29.1.32

Ferrara, M., Terrinoni, A., \& Williams, R. (2012). Non-suicidal self-injury (NSSI) in adolescent inpatients: Assessing personality features and attitude toward death. Child \& Adolescent Psychiatry \& Mental Health, 6(1), 12-19. doi:10.1186/17532000-6-12

Franklin, J., Puzia, M., Lee, K., \& Prinstein, M. (2014). Low implicit and explicit aversion toward self-cutting stimuli longitudinally predict nonsuicidal self-injury. Journal of Abnormal Psychology, 123(2), 463-469. doi:10.1037/a0036436

Gaskin, J. (2012). Data screening. Gaskination's StatWiki. Retrieved from http://statwiki.kolobkreations.com

Gaskin, J. (2013). SEM series part 2: Data screening. Gaskination's Statistics. Retrieved from https://www.youtube.com/watch?v=1KuM5e0aFgU

Gaskin, J. (2016). SEM series (2016) 2. Data screening. Gaskination's Statistics. Retrieved from https://www.youtube.com/watch?v=iWrQ-SgVy-0

Gignac, M., Cott, C., \& Badley, E. (2000). Adaptation to chronic illness and disability and its relationship to perceptions of independence and dependence. The Journals of Gerontology: Series B, 55(6), P362-P372. doi:10.1093/geronb/55.6.P362 
Goldin, P., \& Gross, J. (2010). Effects of mindfulness-based stress reduction (MBSR) on emotion regulation in social anxiety disorder. Emotion, 10(1), 83-91. doi:10.1037/a0018441

Gratz, K., Hepworth, C., Tull, M., Paulson, A., Clarke, S., Remington, B., \& Lejuez, C. (2011). An experimental investigation of emotional willingness and physical pain tolerance in deliberate self-harm: The moderating role of interpersonal distress. Comprehensive Psychiatry, 52(1), 63-74. doi:10.1016/j.comppsych.2010.04.009

Gratz, K., \& Roemer, L. (2004). Multidimensional assessment of emotion regulation and dysregulation: Development, factor structure, and initial validation of the Difficulties in Emotion Regulation Scale. Journal of Psychopathology \& Behavioral Assessment, 26(1), 41-54. doi:10.1007/s10862-008-9102-4

Greenberg, J. (2011). Individual differences: Personality, skills, and abilities (10th ed.). New York, NY: Pearson.

Hajek, P., Belcher, M., \& Stapleton, J. (1987). Breath-holding endurance as a predictor of success in smoking cessation. Addictive Behaviors, 12(3), 285-288. doi:10.1016

Hambrook, D., Oldershaw, A., Rimes, K., Schmidt, U., Tchanturia, K., Treasure, J., . . . Chalder, T. (2011). Emotional expression, self-silencing, and distress tolerance in anorexia nervosa and chronic fatigue syndrome. British Journal of Clinical Psychology, 50(3), 310-325. doi:10.1348/014466510X519215

Hancock, G., \& Mueller, R. (2001). Rethinking construct reliability within latent variable systems. In R. Cudeck, S. du Toit, \& D. Sörbom (Eds.), Structural equation modeling: Present and future (pp. 195-216). Lincoln, IL: Scientific Software International Inc.

Hawkins, K., Macatee, R., Guthrie, W., \& Cougle, J. (2013). Concurrent and prospective relations between distress tolerance, life stressors, and anger. Cognitive Therapy \& Research, 37(3), 434-445. doi:10.1007/s10608-012-9487-y

Hertlein, K., Blumer, M., \& Mihaloliakos, J. (2015). Marriage and family counselors' perceived ethical issues related to online therapy. The Family Journal, 23(1), 512. doi:10.1177/1066480714547184

Hezel, D., \& Hooley, J. (2014). Creativity, personality, and hoarding behavior. Psychiatry Research, 220(1-2), 322-327. doi:10.1016/j.psychres.2014.07.037

Hiroto, D. (1974). Locus of control and learned helplessness. Journal of Experimental Psychology, 102(2), 187-193. doi:10.1037/h0035910

Hiroto, D., \& Seligman, M. (1975). Generality of learned helplessness in man. Journal of Personality and Social Psychology, 31(2), 311-327. doi:10.1037/h0076270 
Hooper, D., Coughlan, J., \& Mullen, M. (2008). Structural equation modelling: Guidelines for determining model fit. Electronic Journal of Business Research Methods, 6(1), 53-60. Retrieved from http://arrow.dit.ie/cgi/viewcontent.cgi?article=1001\&context=buschmanart

Huang, K., Szabó, M., \& Han, J. (2009). The relationship of low distress tolerance to excessive worrying and cognitive avoidance. Behaviour Change, 26(4), 223-234. doi:10.1375/bech.26.4.223

Johnson, K., Berenz, E., \& Zvolensky, M. (2012). Nonclinical panic attack history and anxiety sensitivity: Testing the differential moderating role of self-report and behavioral indices of distress tolerance. Cognitive Therapy \& Research, 36(6), 603-611. doi:10.1007/s10608-011-9410-y

Kapson, H., Leddy, M., \& Haaga, D. (2012). Specificity of effects of cognitive behavior therapy on coping, acceptance, and distress tolerance in a randomized controlled trial for smoking cessation. Journal of Clinical Psychology, 68(12), 1231-1240. doi:10.1002/jclp.21903

Kelly, N., Cotter, E., \& Mazzeo, S. (2014). Examining the role of distress tolerance and negative urgency in binge eating behavior among women. Eating Behaviors, 15(3), 483-489. doi:10.1016/j.eatbeh.2014.06.012

Kenardy, J., Arnow, B., \& Agras, W. (1996). The aversiveness of specific emotional states associated with binge-eating in obese subjects. Austrailian \& New Zealand Journal of Psychiatry, 30(6), 839-844. doi:10.3109/00048679609065053

Keough, M., Riccardi, C., Timpano, K., Mitchell, M., \& Schmidt, N. (2010). Anxiety symptomatology: The association with distress tolerance and anxiety sensitivity. Behavior Therapy, 41(4), 567-574. doi:10.1016/j.beth.2010.04.002

Kertz, S., Stevens, K., McHugh, K., \& Björgvinsson, T. (2015). Distress intolerance and worry: The mediating role of cognitive variables. Anxiety, Stress \& Coping, 28(4), 408-424. doi:10.1080/10615806.2014.974571

Kiselica, A., Webber, T., \& Bornovalova, M. (2014). Stability and change in distress tolerance and its prospective relationship with borderline personality features: A short-term longitudinal study. Personality Disorders, 5(3), 247-256. doi:10.1037/per0000076

Kozak, A., \& Fought, A. (2011). Beyond alcohol and drug addiction. Does the negative trait of low distress tolerance have an association with overeating? Appetite, 57(3), 578-581. doi:10.1016/j.appet.2011.07.008

Kutz, A., Marshall, E., Bernstein, A., \& Zvolensky, M. (2010). Evaluating emotional sensitivity and tolerance factors in the prediction of panic-relevant responding to a 
biological challenge. Journal of Anxiety Disorders, 24(1), 16-22.

doi:10.1016/j.janxdis.2009.07.025

Lampard, A., Byrne, S., McLean, N., \& Fursland, A. (2011). Avoidance of affect in the eating disorders. Eating Behaviors, 12(1), 90-93.

doi:10.1016/j.eatbeh.2010.11.004

Lannoy, S., Heeren, A., Rochat, L., Rossignol, M., Van der Linden, M., \& Billieux, J. (2014). Is there an all-embracing construct of emotion reactivity? Adaptation and validation of the Emotion Reactivity Scale among a French-speaking community sample. Comprehensive Psychiatry, 55(8), 1960-1967.

doi:10.1016/j.comppsych.2014.07.023

Lavender, J., Happel, K., Anestis, M., Tull, M., \& Gratz, K. (2015). The interactive role of distress tolerance and eating expectancies in bulimic symptoms among substance abusers. Eating Behaviors, 16, 88-91. doi:10.1016/j.eatbeh.2014.10.006

Learned helplessness. (2007). In G. VandenBos (Ed.), APA dictionary of psychology (1st ed.). Washington, DC: American Psychological Association.

Leary, M., Tate, E., Adams, C., Allen, A., \& Hancock, J. (2007). Self-compassion and reactions to unpleasant self-relevant events: The implications of treating oneself kindly. Journal of Personality and Social Psychology, 92(5), 887-904. doi:10.1037/0022-3514.92.5.887

Lejuez, C., Banducci, A., \& Long, K. (2013). Commentary on the distress tolerance special issue. Cognitive Therapy \& Research, 37(3), 510-513.

doi:10.1007/s10608-013-9527-2

Lejuez, C., Kahler, C., \& Brown, R. (2003). A modified computer version of the Paced Auditory Serial Addition Task (PASAT) as a laboratory-based stressor. The Behavior Therapist, 26(4), 290-293. Retrieved from http://psycnet.apa.org/psycinfo/2003-06608-006

Levine, J., Irving, K., Brooks, J., \& Fishman, G. (1993). Group therapy and the somatoform patient: An integration. Psychotherapy: Theory, Research, Practice, Training, 30(4), 625-634. doi:10.1037/0033-3204.30.4.625

Leyro, T., Berenz, E., Brandt, C., Smits, J., \& Zvolensky, M. (2012). Evaluation of perseveration in relation to panic-relevant responding: An initial test. Behavioural and Cognitive Psychotherapy, 40(2), 205-219. doi:10.1017/S135246581100066X

Leyro, T., Zvolensky, M., \& Bernstein, A. (2010). Distress tolerance and psychopathological symptoms and disorders: A review of the empirical literature among adults. Psychological Bulletin, 136(4), 576-600. doi:10.1037/a0019712 
Linehan, M. (2015). DBT skills training manual (2nd ed.). New York, NY: Guilford Press.

Lisle, S., Dowling, N., \& Allen, J. (2014). Mechanisms of action in the relationship between mindfulness and problem gambling behaviour. International Journal of Mental Health \& Addiction, 12(2), 206-225. doi:10.1007/s11469-014-9475-4

Lovibond, S., \& Lovibond, P. (1995). Manual for the Depression Anxiety Stress Scales. Sydney, Australia: Psychology Foundation of Australia.

Lydecker, J., Hubbard, R., Tully, C., Utsey, S., \& Mazzeo, S. (2014). White public regard: Associations among eating disorder symptomatology, guilt, and white guilt in young adult women. Eating Behaviors, 15(1), 76-82.

doi:10.1016/j.eatbeh.2013.10.007

Lynch, T., \& Mizon, G. (2011). Distress overtolerance and distress intolerance: A behavioral perspective. In M. Zvolensky, A. Bernstein, \& A. Vujanovic (Eds.), Distress tolerance: Theory, research, and clinical applications (pp. 52-79). New York, NY: Guilford Press.

Macatee, R., Capron, D., Guthrie, W., Schmidt, N., \& Cougle, J. (2015). Distress tolerance and pathological worry: Tests of incremental and prospective relationships. Behavior Therapy, 46(4), 449-462. doi:10.1016/j.beth.2015.03.003

Macatee, R., \& Cougle, J. (2013). The roles of emotional reactivity and tolerance in generalized, social, and health anxiety: A multimethod exploration. Behavior Therapy, 44(1), 39-50. doi:10.1016/j.beth.2012.05.006

Macatee, R., \& Cougle, J. (2015). Development and evaluation of a computerized intervention for low distress tolerance and its effect on performance on a neutralization task. Journal of Behavior Therapy \& Experimental Psychiatry, 48, 33-39. doi:10.1016/j.jbtep.2015.01.007

Maier, S., \& Seligman, M. (2016). Learned helplessness at fifty: Insights from neuroscience. Psychological Review, 123(4), 349-367. doi:10.1037/rev0000033

Marshall, E., Zvolensky, M., Vujanovic, A., Gregor, K., Gibson, L., \& Leyro, T. (2008). Panic reactivity to voluntary hyperventilation challenge predicts distress tolerance to bodily sensations among daily cigarette smokers. Experimental and Clinical Psychopharmacology, 16(4), 313-321. doi:10.1037/a0012752

Mason, W., \& Suri, S. (2012). Conducting behavior research on Amazon's Mechanical Turk. Behavioral Research, 44, 1-23. doi:10.3758/s13428-011-0124-6

McHugh, K., Kertz, S., Weiss, R., Baskin-Sommers, A., Hearon, B., \& Björgvinsson, T. (2014). Changes in distress intolerance and treatment outcome in a partial hospital setting. Behavior Therapy, 45(2), 232-240. doi:10.1016/j.beth.2013.11.002 
McHugh, K., \& Otto, M. (2012). Refining the measurement of distress intolerance. Behavior Therapy, 43(3), 641-651. doi:10.1016/j.beth.2011.12.001

McHugh, K., Reynolds, E., Leyro, T., \& Otto, M. (2013). An examination of the association of distress intolerance and emotion regulation with avoidance. Cognitive Therapy \& Research, 37(2), 363-367. doi:10.1007/s10608-012-9463-6

McKeever, V., McWhirter, B., \& Huff, M. (2006). Relationships between attribution style, child abuse history, and PTSD symptom severity among Vietnam veterans. Cognitive Therapy \& Research, 30, 123-133. doi:10.1007/s10608-006-9018-9

Mitchell, G., \& Locke, K. (2015). Lay beliefs about autism spectrum disorder among the general public and childcare providers. Autism, 19(5), 553-561. doi:10.1177/1362361314533839

Mitchell, M., Riccardi, C., Keough, M., Timpano, K., \& Schmidt, N. (2013). Understanding the associations among anxiety sensitivity, distress tolerance, and discomfort intolerance: A comparison of three models. Journal of Anxiety Disorders, 27(1), 147-154. doi:10.1016/j.janxdis.2012.12.003

Mountford, V., Corstorphine, E., Tomlinson, S., \& Waller, G. (2007). Development of a measure to assess invalidating childhood environments in the eating disorders. Eating Behaviors, 8(1), 48-58. doi:10.1016/j.eatbeh.2006.01.003

Muehlenkamp, J., \& Kerr, P. (2010). Untangling a complex web: How non-suicidal selfinjury and suicide attempts differ. The Prevention Researcher, 17(1), 8-10. Retrieved from http://search.ebscohost.com/login.aspx?direct=true \&db=ccm\&AN=2010565285\& site $=$ ehost-live

Nation, J., \& Massad, P. (1978). Persistence training: A partial reinforcement procedure for reversing learned helplessness and depression. Journal of Experimental Psychology General, 107(4), 436-451.

Nguyen-Feng, V., Frazier, P., Greer, C., Howard, K., Paulsen, J., Meredith, L., \& Kim, S. (2015). A randomized controlled trial of a web-based intervention to reduce distress among students with a history of interpersonal violence. Psychology of Violence, 5(4), 444-454. doi:10.1037/a0039596

Nock, M., Holmberg, E., Photos, V., \& Michel, B. (2007). Self-Injurious Thoughts and Behaviors Interview: Development, reliability, and validity in an adolescent sample. Psychological Assessment, 19(3), 309-317. doi:10.1037/10403590.19.3.309

Nock, M., \& Mendes, W. (2008). Physiological arousal, distress tolerance, and social problem-solving deficits among adolescent self-injurers. Journal of Consulting and Clinical Psychology, 76(1), 11. doi:10.1037/0022-006X.76.1.28 
Nock, M., Park, J., Finn, C., Deliberto, T., Dour, H., \& Banaji, M. (2010). Measuring the suicidal mind: Implicit cognition predicts suicidal behavior. Psychological Science, 21(4), 511-517. doi:10.1177/0956797610364762

Nock, M., Wedig, M., Holmberg, E., \& Hooley, J. (2008). The Emotion Reactivity Scale: Development, evaluation, and relation to self-injurious thoughts and behaviors. Behavior Therapy, 39(2), 107-116. doi:10.1016/j.beth.2007.05.005

Norr, A., Allan, N., Macatee, R., Keough, M., \& Schmidt, N. (2014). The effects of an anxiety sensitivity intervention on anxiety, depression, and worry: Mediation through affect tolerances. Behaviour Research and Therapy, 59, 12-19. doi:10.1016/j.brat.2014.05.011

Norr, A., Oglesby, M., Capron, D., Raines, A., Korte, K., \& Schmidt, N. (2013). Evaluating the unique contribution of intolerance of uncertainty relative to other cognitive vulnerability factors in anxiety psychopathology. Journal of Affective Disorders, 151(1), 136-142. doi:10.1016/j.jad.2013.05.063

O'Donnell, S. (2006). Learned helplessness. In N. Salkind (Ed.), Encyclopedia of Human Development (pp. 778-780). Thousand Oaks, CA: Sage.

Oldershaw, A., DeJong, H., Hambrook, D., Broadbent, H., Tchanturia, K., Treasure, J., \& Schmidt, U. (2012). Emotional processing following recovery from anorexia nervosa. European Eating Disorders Review, 20(6), 502-509. doi:10.1002/erv.2153

Pearson, M., Lawless, A., Brown, D., \& Bravo, A. (2015). Mindfulness and emotional outcomes: Identifying subgroups of college students using latent profile analysis. Personality and Individual Differences, 76, 33-38. doi:10.1016/j.paid.2014.11.009

Peterson, C. (2010). Learned helplessness. In I. Weiner \& W. Craighead (Eds.), The Corsini encyclopedia of psychology (4th ed., Vol. 2, pp. 917-919). Hoboken, NJ: John Wiley \& Sons.

Peterson, C., Davis-Becker, K., \& Fischer, S. (2014). Interactive role of depression, distress tolerance and negative urgency on non-suicidal self-injury. Personality \& Mental Health, 8(2), 151-160. doi:10.1002/pmh.1256

Peterson, C., Maier, S., \& Seligman, M. (1993). Learned helplessness: A theory for the age of personal control. New York, NY: Oxford University Press.

Peterson, C., \& Seligman, M. (2004). Character strengths and virtues: A handbook and classification. New York, NY: Oxford University Press.

Porter, G., Starcevic, V., Berle, D., \& Fenech, P. (2010). Recognizing problem video game use. Austrailian \& New Zealand Journal of Psychiatry, 44(2), 120-128. doi:10.3109/00048670903279812 
Proudfoot, J., Corr, P., Guest, D., \& Dunn, G. (2009). Cognitive-behavioural training to change attributional style improves employee well-being, job satisfaction, productivity, and turnover. Personality and Individual Differences, 46(2), 147153. doi:10.1016/j.paid.2008.09.018

Purington, A., \& Whitlock, J. (2010). Non-suicidal self-injury in the media. The Prevention Researcher, 17(1), 11-13. Retrieved from http://search.ebscohost.com/login.aspx?direct=true \&db=ccm\&AN=2010565286\& site=ehost-live

QuestionPro. (2016). Simple. Powerful. Survey software. Retrieved from www.questionpro.com

Quinless, F., \& Nelson, M. (1988). Development of a measure of learned helplessness. Nursing Research, 37(1).

Quinn, E., Brandon, T., \& Copeland, A. (1996). Is task persistence related to smoking and substance abuse? The application of learned industriousness theory to addictive behaviors. Experimental and Clinical Psychopharmacology, 4(2), 186190. doi:10.1037/1064-1297.4.2.186

Raykos, B., Byrne, S., \& Watson, H. (2009). Confirmatory and exploratory factor analysis of the Distress Tolerance Scale (DTS) in a clinical sample of eating disorder patients. Eating Behaviors, 10(4), 215-219. doi:10.1016/j.eatbeh.2009.07.001

Raykov, T., \& Marcoulides, G. (2006). A first course in structural equation modeling (2nd ed.). Mahwah, NJ: Lawrence Erlbaum Associates.

Richards, C., \& Perri, M. (Eds.). (2010). Relapse prevention for depression. Washington, DC: American Psychological Association.

Rubenstein, L., Freed, R., Shapero, B., Fauber, R., \& Alloy, L. (2016). Cognitive attributions in depression: Bridging the gap between research and clinical practice. Journal of Psychotherapy Integration, 26, 103-115. doi:10.1037/int0000030

Rutherford, H., Booth, C., Luyten, P., Bridgett, D., \& Mayes, L. (2015). Investigating the association between parental reflective functioning and distress tolerance in motherhood. Infant Behavior \& Development, 40, 54-63. doi:10.1016/j.infbeh.2015.04.005

Sargeant, M., Daughters, S., Curtin, J., Schuster, R., \& Lejuez, C. (2011). Unique roles of antisocial personality disorder and psychopathic traits in distress tolerance. Journal of Abnormal Psychology, 120(4), 987-992. doi:10.1037/a0024161 
Sayama, M., \& Sayama, H. (2011). Positive stereotyping and multicultural awareness: An online experiment. Current Directions in Social Psychology, 16(6). Retrieved from https://uiowa.edu/crisp/sites/uiowa.edu.crisp/files/16.6.pdf

Schloss, H., \& Haaga, D. (2011). Interrelating behavioral measures of distress tolerance with self-reported experiential avoidance. Journal of Rational-Emotive And Cognitive-Behavior Therapy, 29(1), 53-63. doi:10.1007/s10942-011-0127-3

Schmidt, N., Mitchell, M., Keough, M., \& Riccardi, C. (2011). Anxiety and its disorders. In M. Zvolensky, T. Leyro, A. Bernstein, \& A. Vujanovic (Eds.), Distress tolerance: Theory, research, and clinical applications (pp. 105-125). New York, NY: Guilford Press.

Schmidt, N., Richey, A., \& Fitzpatrick, K. (2006). Discomfort intolerance: Development of a construct and measure relevant to panic disorder. Journal of Anxiety Disorders, 20(3), 263-280. doi:10.1016/j.janxdis.2005.02.002

Schoenefeld, S., \& Webb, J. (2013). Self-compassion and intuitive eating in college women: Examining the contributions of distress tolerance and body image acceptance and action. Eating Behaviors, 14(4), 493-496. doi:10.1016/j.eatbeh.2013.09.001

Shaffer, D., \& Jacobson, C. (2010). Proposal to the DSM-V childhood disorder and mood disorder work groups to include non-suicidal self-injury (NSSI) as a DSM-V disorder. Retrieved from www.dsm5.org

Shea, D. (2008). Effects of sexual abuse by Catholic priests on adults victimized as children. Sexual Addiction and Compulsivity, 15, 250-268. doi:10.1080/10720160802288993

Shorey, R., Febres, J., Brasfield, H., Zucosky, H., Cornelius, T., \& Stuart, G. (2013). Reactions to dating violence research: Do difficulties with distress tolerance increase negative reactions? Journal of Family Violence, 28(5), 479-487. doi:10.1007/s10896-013-9519-8

Simons, J., \& Gaher, R. (2005). The Distress Tolerance Scale: Development and validation of a self-report measure. Motivation \& Emotion, 29(2), 83-102. doi:10.1007/s11031-005-7955-3

Skinner, E. (1995). Perceived control, motivation, \& coping. Thousand Oaks, CA: Sage.

Slee, N., Garnefski, N., Spinhoven, P., \& Arensman, E. (2008). The influence of cognitive emotion regulation strategies and depression severity on deliberate selfharm. Suicide and Life-Threatening Behavior, 38(3), 274-286. doi:10.1521/suli.2008.38.3.274. 
Strigo, I., Simmons, A., Matthews, S., Craig, A., \& Paulus, M. (2008). Association of major depressive disorder with altered functional brain response during anticipation and processing of heat pain. Archives of General Psychiatry, 65(11), 1275-1284. doi:10.1001/archpsyc.65.11.1275

Strong, D., Lejuez, C., Daughters, S., Marinello, M., Kahler, C., \& Brown, R. (2003). The computerized mirror tracing task, version 1. Unpublished manual. Retrieved from http://www.addiction.umd.edu/research.htm

Substance Abuse and Mental Health Services Administration. (2011). The treatment of depression in older adults: Selecting evidence-based practices for treatment of depression in older adults. (HHS Pub. No. SMA-11-4631). Rockville, MD: Center for Mental Health Services, Substance Abuse and Mental Health Services Administration, U.S. Department of Health and Human Services.

Survey Analytics. (2010). Survey Analytics 2010 Panel Book. Retrieved from http://www.questionpro.com/resources/SurveyAnalytics-PanelBook.html

Sweeney, P., Anderson, K., \& Bailey, S. (1986). Attributional style in depression: A meta-analytic review. Journal of Personality and Social Psychology, 50(5), 974991.

Trafton, J., \& Gifford, E. (2011). Biological bases of distress tolerance. In M. Zvolensky, A. Bernstein, \& A. Vujanovic (Eds.), Distress tolerance: Theory, research, and clinical applications (pp. 80-102). New York, NY: Guilford Press.

United States Census Bureau. (2014). QuickFacts. Retrieved from https://www.census.gov/quickfacts/table/PST045215/00

Van Camp, I., Desmet, M., \& Verhaeghe, P. (2011). Gender difference in non-suicidal self injury: Are they on the verge of leveling off? International Proceedings of Economics Development and Research, 23, 28-34. Retrieved from http://connection.ebscohost.com/c/articles/74274052/gender-differences-nonsuicidal-self-injury-are-they-verge-leveling-off

Van Eck, K., Flory, K., \& Willis, D. (2015). Does distress intolerance moderate the link between ADHD symptoms and number of sexual partners? Attention Deficit And Hyperactivity Disorders, 7(1), 39-47. doi:10.1007/s12402-014-0140-3

Vujanovic, A., Marshall-Berenz, E., \& Zvolensky, M. (2011). Posttraumatic stress and alcohol use motives: A test of the incremental and mediating role of distress tolerance. Journal of Cognitive Psychotherapy, 25(2), 130-141. doi:10.1891/0889-8391.25.2.130

Washburn, J., Richardt, S., Styer, D., Gebhardt, M., Juzwin, K., Yourek, A., \& Aldridge, D. (2012). Psychotherapeutic approaches to non-suicidal self-injury in 
adolescents. Child \& Adolescent Psychiatry \& Mental Health, 6(1), 14-21. doi:10.1186/1753-2000-6-14

Webb, J., \& Forman, M. (2013). Evaluating the indirect effect of self-compassion on binge eating severity through cognitive-affective self-regulatory pathways. Eating Behaviors, 14(2), 224-228. doi:10.1016/j.eatbeh.2012.12.005

Weems, C. (2011). Anxiety sensitivity as a specific form of distress tolerance in youth: Developmental assessment, origins, and applications. In M. Zvolensky, T. Leyro, A. Bernstein, \& A. Vujanovic (Eds.), Distress tolerance: Theory, research, and clinical applications (pp. 28-51). New York, NY: Guilford Press.

Weitzman, M., McHugh, K., \& Otto, M. (2011). The association between affect amplification and urgency. Depression \& Anxiety (1091-4269), 28(12), 11051110. doi:10.1002/da.20830

Whitlock, J., Muehlenkamp, J., Purington, A., Eckenrode, J., Barreira, P., Abrams, G., . . . Knox, K. (2011). Nonsuicidal self-injury in a college population: General trends and sex differences. Journal of American College Health, 59(8), 691-698. doi:10.1080/07448481.2010.529626

Whitlock, J., Pietrusza, C., \& Purington, A. (2013). Young adult respondent experiences of disclosing self-injury, suicide-related behavior, and psychological distress in a web-based survey. Archives of Suicide Research, 17(1), 20-32. doi:10.1080/13811118.2013.748405

Williams, A. (2012). Distress tolerance and experiential avoidance in compulsive acquisition behaviours. Australian Journal of Psychology, 64(4), 217-224. doi:10.1111/j.1742-9536.2012.00055.x

Williams, A., Thompson, J., \& Andrews, G. (2013). The impact of psychological distress tolerance in the treatment of depression. Behaviour Research \& Therapy, 51(8), 469-475. doi:10.1016/j.brat.2013.05.005

Winward, J., Bekman, N., Hanson, K., Lejuez, C., \& Brown, S. (2014). Changes in emotional reactivity and distress tolerance among heavy drinking adolescents during sustained abstinence. Alcoholism: Clinical \& Experimental Research, 38(6), 1761-1769. doi:10.1111/acer.12415

Yamamoto, K., Kanbara, K., Mutsuura, H., Ban, I., Mizuno, Y., Abe, T., . . Fukunaga, M. (2010). Psychological characteristics of Japanese patients with chronic pain assessed by the Rorschach test. Biopsychosocial Medicine, 4, 20-20. doi:10.1186/1751-0759-4-20

Zautra, A., Davis, M., Reich, J., Sturgeon, J., Arewasikporn, A., \& Tennen, H. (2012). Phone-based interventions with automated mindfulness and mastery messages 
improve the daily functioning for depressed middle-aged community residents. Journal of Psychotherapy Integration, 22(3), 206-228. doi:10.1037/a0029573

Zvolensky, M., Feldner, M., Eifert, G., \& Brown, R. (2001). Affective style among smokers: Understanding anxiety sensitivity, emotional reactivity, and distress tolerance using biological challenge. Addictive Behaviors, 26(6), 901-915. doi:10.1016/S0306-4603(01)00242-8

Zvolensky, M., Leyro, T., Bernstein, A., \& Vujanovic, A. (2011). Historical perspectives, theory, and measurement of distress tolerance. In M. Zvolensky, T. Leyro, A. Bernstein, \& A. Vujanovic (Eds.), Distress tolerance: Theory, research, and clinical applications (pp. 3-27). New York, NY: Guilford Press.

Zvolensky, M., Vujanovic, A., Bernstein, A., \& Leyro, T. (2010). Distress tolerance: Theory, measurement, and relations to psychopathology. Current Directions in Psychological Science, 19(6), 406-410. doi:10.1177/0963721410388642 
VITA 


\section{VITA}

NAME: $\quad$ Brittany Kay Sommers

DATE OF BIRTH: $\quad$ May 11, 1990

EDUCATION: $\quad$ Doctor of Philosophy, Counseling Psychology, 2017

Andrews University, Berrien Springs, Michigan

Master of Science, Counseling Psychology, 2015

Andrews University, Berrien Springs, Michigan

Bachelor of Arts, Psychology, Summa Cum Laude, 2011

Huntington University, Huntington, Indiana

PROFESSIONAL EXPERIENCE:

2016 - present Doctoral Psychology Intern

Cleveland State University Counseling Center

Cleveland, Ohio

2015 - $2016 \quad$ Staff Counselor

2014 - $2015 \quad$ Doctoral Practicum Counselor

Southwestern Medical Clinic Counseling \& Psychological Services

Stevensville, Michigan

2014 - $2016 \quad$ Counseling Group Facilitator

Well of Grace Ministries

Stevensville, Michigan

2012 - $2016 \quad$ Clinic Coordinator

$2015 \quad$ Masters Practicum Supervisor

2013 - $2014 \quad$ Doctoral Practicum Counselor

Andrews Community Counseling Center

Berrien Springs, Michigan

\section{PUBLICATIONS:}

Grajales, T., Sommers, B. (2016). Identity Styles and Religiosity: Examining the Role of Identity Commitment. Journal of Research on Christian Education, 25(2), 188-202. doi:10.1080/10656219.2016.1191394

Grajales, T., Leon, V., Al Nasser, F., Hooley, E., Sample, M., Sommers, B. (2016). Examining Identity Styles and Religiosity among Chilean Undergraduate Students. Journal of Research on Christian Education, 25(3), 290-301. doi:10.1080/10656219.2016.1238327 